\title{
HETEROMETALLIC TIN COMPOUNDS: CLASSIFICATION AND ANALYSIS OF CRYSTALLOGRAPHIC AND STRUCTURAL DATA: PART II. TRIMERIC TO POLYMERIC DERIVATIVES
}

\author{
Clive E. Holloway* ${ }^{* 1}$ and Milan Melnik ${ }^{2}$
}

${ }^{1}$ Department of Chemistry, York University, 4700 Keele St., North York, M3J IP3. Ontario, Canada

${ }^{2}$ Department of Inorganic Chemistry, Slovak Technical University, SK 81237. Bratislava, Slovak Republic

\begin{abstract}
Over 380 heterometallic tin compounds include 130 trimers, 100 tetramers, 30 pentamers, 45 hexamers. 15 heptamers, 12 octamers, 20 oligomers and 30 polymers. In these the $\mathrm{Sn}$ atom is found in oxidation states of $0,+2$ and +4 , with geometries including trigonal planar, tetrahedral, trigonal bipyramidal, octahedral and seven coordinate, with varying degrees of distortion. Of these, the first only exhibits $\mathrm{Sn}(0)$ and $\mathrm{Sn}(\mathrm{II})$ and the last only Sn(II) oxidation states, with tetrahedral geometry the most common overall. Examples of distortion isomersim are reported, with many being exhibited within the same crystal. The heterometal atoms include the transition and lanthanide metals along with the non-transition metals. Outside the dimers. the shortest Sn-M(non-transition) bond distances are found in the tetramers and hexamers. Both Sn(IV) and Sn(II) are found bonded to a variety of "hard" and "soft" heterometal atoms. Comparisons are made with the much less rich chemistry of $\mathrm{Pb}$.
\end{abstract}

\section{CONTENTS}

0 ABBREVIATIONS

1. INTRODUCTION

2. TRIMERIC COMPOUNDS

3. TETRAMERIC COMPOUNDS

4. PENTAMERIC COMPOUNDS

5. HEXAMERIC COMPOUNDS

6. HEPTAMERIC COMPOUNDS

7. OCTAMERIC COMPOUNDS

8. OLIGOMERIC COMPOUNDS

9. POLYMERIC COMPOUNDS

10. CONCLUSIONS

11. ACKNOWLEDGEMENTS

12. REFERENCES

\begin{tabular}{|c|c|}
\hline 0. & BREVIATTIONS \\
\hline ac & acetate \\
\hline acac & acetylacetonate \\
\hline ahb & o-(amino-_-hydroxybenzylidenehydrazonomethyl)phenyl \\
\hline ampy & 2-amino-6-methylpyridine \\
\hline bpy & 2,2'-bipyridine \\
\hline $\mathrm{Bu}$ & tert-butyl \\
\hline $\mathrm{Bu}^{\mathrm{t}} \mathrm{b} \mathrm{bpv}$ & 4,4'-di-tert-butyl-2,2'-bipyridine \\
\hline $\mathrm{CH}_{3} \mathrm{C}_{5} \mathrm{H}_{4}$ & methylcyclopentadienyl \\
\hline $\mathrm{C}_{4} \mathrm{H}_{7}$ & methylallyl \\
\hline $\mathrm{C}_{5} \mathrm{H}_{7} \mathrm{O}_{2}$ & 2,4-pentānedionate \\
\hline $\mathrm{C}_{6} \mathrm{H}_{6}$ & benzene \\
\hline $\mathrm{C}_{6} \mathrm{H}_{10}$ & 2,3-dimethylbuta-1,3-diene \\
\hline $\mathrm{C}_{7} \mathrm{H}_{8}$ & bicyclo[2.2.1]heptadiene \\
\hline $\mathrm{C}_{8} \mathrm{H} \circ \mathrm{NC}$ & xylylisocyanide \\
\hline $\mathrm{C}_{8} \mathrm{H}_{10}$ & naphthalene \\
\hline $\mathrm{C}_{20} \mathrm{H}_{14}$ & triptycene \\
\hline & citrate \\
\hline $\operatorname{cod}$ & 1,5-cyclooctadiene \\
\hline $\mathrm{cp}^{*}$ & pentamethylcyclopentadienyl \\
\hline
\end{tabular}




\begin{tabular}{|c|c|}
\hline cpy & $2-\left\{\left(\mathrm{Me}_{3} \mathrm{Si}\right){ }_{2} \mathrm{C}\right\} \mathrm{C}_{5} \mathrm{H}_{4} \mathrm{~N}$ \\
\hline cy & cyclohexyl \\
\hline $\mathrm{db}$ & 1,3-diborolenyl \\
\hline dedtc & $\mathrm{N}, \mathrm{N}$-diethyldithiocarbamate \\
\hline $\begin{array}{l}\text { 1,4-dio } \\
\text { dm }\end{array}$ & $\begin{array}{l}\text { 1,4-dioxane } \\
\text { dioximate }\end{array}$ \\
\hline dmso & dimethylsulphoxide \\
\hline dmdtc & $\mathrm{N}, \mathrm{N}$-dimethyldithiocarbamate \\
\hline $\begin{array}{l}\text { dpma } \\
\text { dppb }\end{array}$ & $\begin{array}{l}\text { bis(diphenylphosphinomethyl)phenylarsine } \\
\text { l,4-bis(diphenylphosphine)butane }\end{array}$ \\
\hline dppm & $\begin{array}{l}\text { 1,4-0is(alpnenylpnospnine)outane } \\
\text { bis(diphenylphosphine)methylene }\end{array}$ \\
\hline dto & dithioxalate \\
\hline en & ethylenediamine \\
\hline fu & furyl \\
\hline hfb & hexafluorobut-2-yne \\
\hline imn & 1-imidonaphthyl \\
\hline m & monoclinic \\
\hline ma & $2-\mathrm{MeOC}_{6} \mathrm{H}_{4}$ \\
\hline & methyl \\
\hline$\left(\mathrm{Me} \mathrm{Si}_{2} \mathrm{P}\right.$ & bis(trimethylsilyl)phosphanide \\
\hline mes & mesityl \\
\hline mit & N-methyl-imidazole-2-thio \\
\hline nep & 2,2-dimethylpropyl \\
\hline $\mathbf{n x}$ & nioximate \\
\hline or & orthorhombic \\
\hline 8-OHqu & 8-hydroxyquinolate \\
\hline & phenyl \\
\hline$(\mathrm{Ph} 2 \mathrm{P})_{2} \mathrm{py}$ & $\begin{array}{l}\text { 2,6-bis(diphenylphosphino)pyridine } \\
\text { 2-phenvl-2-propanolate }\end{array}$ \\
\hline phen & 1,10-phenanthroline \\
\hline $\mathrm{PMe}_{3}$ & trimethylphosphine \\
\hline $\mathrm{PPh}_{3}$ & triphenylphosphine \\
\hline ppn & $\left(\mathrm{Ph}{ }_{3} \mathrm{P}\right) \mathrm{N}^{+*}$ \\
\hline $\begin{array}{l}\operatorname{Pr}_{2}^{2} \text { dab } \\
\text { Ptols }_{3}\end{array}$ & $\begin{array}{l}\text { N,N'-diisopropyl-1,4-diaza-1,3-butadiene } \\
\text { tritolylphosphine }\end{array}$ \\
\hline $\mathrm{pz}$ & pyrazolyl \\
\hline pzSs & porphyrazine- $2,3,7,8,12,13,17,18$-octathiolate \\
\hline & $2,4,6-\left\{\left(\mathrm{Me}_{3} \mathrm{Si}\right)_{2} \mathrm{CH}\right\}_{3} \mathrm{C}_{6} \mathrm{H}_{2}$ \\
\hline tedip & $(\mathrm{EtO})_{2} \mathrm{POP}(\mathrm{OEt})_{2}$ \\
\hline $\begin{array}{l}\text { tepid } \\
\text { tfmp }\end{array}$ & $\begin{array}{l}\text { tri-isopropylphosphoniodithioformate } \\
\text { 1,3,5-tris(trifluoromethyl)phenyl }\end{array}$ \\
\hline thf & tetrahydrofuran \\
\hline tip & $2,4,6-\left(\mathrm{Me}_{2} \mathrm{C}\right)_{3} \mathrm{C}_{6} \mathrm{H}_{2}$ \\
\hline tol & toluene \\
\hline tpsp & tri-iso-propylsilylphosphanide \\
\hline tpspi & tri-iso-propylsilylphosphandiide \\
\hline tpp & $5,10,15,20$-tetraphenylporphyrinate \\
\hline & triclinic \\
\hline
\end{tabular}

\section{INTRODUCTION}

The last thirty years have seen many studies on the reaction of various metal salts with tin salts. In many cases compounds have been obtained for which crystallographic and structural data could be obtained. This review covers some 370 heterotin cluster complexes from trimers to polymers and complements a previous review of about 240 dimeric heterotin compounds [1]. Previous reviews have also covered some 600 tin coordination compounds [2], over 1,000 monomeric organotin compounds [3], over 260 dimeric organotin compounds [4] and almost 400 higher oligomers of organtin compounds [5]. The structures in this review are classified according to nuclearity, and within each subgroup by the increasing number of heterometal atoms. The factors relevant to the stereochemical interactions around these metals are discussed, and where appropriate 
comparisons are made with other cluster systems.

\section{TRIMERIC COMPOUNDS}

There are almost 130 heterometallic tin compounds shown in Table 1 , mostly with quite complex structures. Fifty five of these have two tin atoms and one heterometal atom, another sixty have one tin atom and two heterometal atoms, and eight contain one tin and two different heterometal atoms. These are listed in the Table by increasing number of heterometal atom and further subdivided into non-transition, transition and lanthanide compounds, and finally by increasing Sn-M distance.

\subsection{Two Sn plus $M$}

\subsubsection{Non-Transition Metal $M$}

A colourless $\mathrm{Sn}_{2} \mathrm{Li}$ cluster [6] contains a [ $\left.\mathrm{Li}(1,4 \text {-dioxane })_{4}\right]^{+}$cation and a $\left[\mathrm{Sn}(\mu \text {-furyl })_{3} \mathrm{Li}(\mu \text {-furyl })_{3} \mathrm{Sn}\right]^{-}$ anion. In the latter the central $\mathrm{Li}$ atom has six furyl $\mathrm{O}$ atoms in an octahedral arrangement $\left(\mathrm{LiO}_{6}\right)$. Each satellite $\mathrm{Sn}$ (II) atom is bonded by three furyl carbon atoms in a typical pyramidal arrangement with C-Sn-C angles of 96(2)

There are four isostructural derivatives in which the heterometal atom is divalent $\mathrm{Ca}, \mathrm{Pb}$ or $\mathrm{Cd}$ [7], or $\mathrm{Sr}$ [9], which have the composition [ $\mathrm{Sn}(\mu-\mathrm{OBu})_{3} \mathrm{M}(\mu-\mathrm{OBu})_{3} \mathrm{Sn}$ ]. In all cases the central $\mathrm{M}$ (II) atom is six coordinate $\left(\mathrm{MO}_{6}\right)$ while the outer $\mathrm{Sn}(\mathrm{Il})$ atoms are three coordinate $\left(\mathrm{SnO}_{3}\right)$. The $\mathrm{SnO}_{3} \mathrm{MO}_{3} \mathrm{Sn}$ framework is surrounded by six bulky tertiary butyl groups. The Sn-M and M-O distances increase as the ionic radius of $\mathrm{M}$ (II) increases, for example: $312.1(3)$ and $234(1) \mathrm{pm}(\mathrm{M}=\mathrm{Cd}, 95 \mathrm{pm})<313.3(3)$ and $237(1) \mathrm{pm}(\mathrm{Ca}, 100 \mathrm{pm})<$ $331.9(1)$ and $252.3(3) \mathrm{pm}(\mathrm{Sr}, 118 \mathrm{pm})<336.1(1)$ and $256.0(9) \mathrm{pm}(\mathrm{Pb}, 119 \mathrm{pm})$. By contrast, the Sn-O values remain almost constant at $208 \mathrm{pm}$. Another colourless $\mathrm{Sn}_{2} \mathrm{Ca}$ derivative [8] has the calcium atom on the crystallographic centre of inversion. It has a distorted octahedral coordination by two tin atoms ( $\mathrm{Sn}-\mathrm{Ca}, 327.2(3)$ pm) and four oxygen atoms from the (thf) ligands in a trans configuration. The Sn(II) atoms are also coordinated to three methyl groups. Barium is the "central" atom in another derivative [10] and is linked to four P atoms of $\mathrm{P}\left(\mathrm{SiMe}_{3}\right)_{2}$ groups which also bridge to the two outer $\mathrm{Sn}$ (II) atoms. These are also coordinated by terminal $\mathrm{P}\left(\mathrm{SiMe}_{3}\right)_{2}$ groups to give a pyramidal $\mathrm{SnP}_{3}$ geometry. Finally, $\mathrm{Pb}$ is found in an "approximate" mirror plane of a Snz $\mathrm{Pb}$ derivative along with a $\mu_{3}-0$ xo bridge. The plane also bisects the Sn- $\mu_{3} \mathrm{O}-\mathrm{Sn}^{\prime}$ link. The complex contains four syn-syn acetate ligands and four tert-butoxy ligands, two bridging and two terminal.

\subsubsection{Transition or Lanthanide Metal M}

Four coloured Sn2Ti derivatives have titanium as the "central" metal atom [12-15]. The red derivative [12] consists of $\mathrm{NEt}_{4}{ }^{+}$cations and $\left[\mathrm{Ph}_{3} \mathrm{SnTiSnPH}_{3}(\mathrm{CO})_{s}\right]^{-2}$ anions, the latter having Ti(0) coordinated by five carbonyl groups and two Sn(II) atoms making it seven coordinate (Sn-Ti-Sn angle, 141.07(6) ${ }^{\circ}$. The Sn(II) atoms have tetrahedral arrangements composed of a titanium atom (Sn-Ti, 281.1(2) and 284.1(2) pm) and three phenyl groups $\left(\mathrm{SnC}_{2} \mathrm{Ti}\right)$. A black derivative [13] has $\mathrm{Ti}(\mathrm{O})$ coordinated by two $\eta-\mathrm{C}_{10} \mathrm{H}_{8}$ groups and two $\mathrm{Sn}$ (II) atoms. The Sn-Ti distances are 286.5(3) and 286.9(3) pm, which are longer than those in the red example [12]. The Sn(II) atoms have a tetrahedral geometry with a Ti atom and three methyl groups (Sn-Ti-Sn', 76.44(6) $)$. A

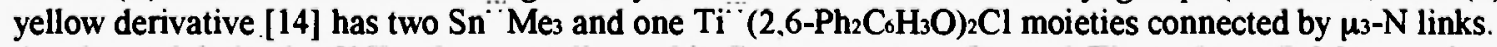
Another red derivative [15] has crystallographic $\mathrm{C}_{2}$ symmetry. One $\mathrm{cp}_{2}{ }_{2} \mathrm{Ti}$ and two $\mathrm{SnMe}_{3}$ units are perpendicularly bridged by an acetylene group. Each $\mathrm{Sn}(\mathrm{IV})$ atom has tetrahedral geometry ( $\left.\mathrm{SnC}_{4}\right)$, and the Ti(II) atom sits in a sandwich (cp) which incorporates both carbon atoms of the acetylene group.

A dark red $\mathrm{Sn}_{2} \mathrm{Zr}$ derivative [16] shows a $\mathrm{Zr}$ (II) central atom with pseudo-tetrahedral geometry from two stannylene donors, with a 2-fold axis bisecting the cp-Zr-cp and $\mathrm{Sn}-\mathrm{Zr}-\mathrm{Sn}^{\prime}$ angles. $\mathrm{The} \mathrm{CH}\left(\mathrm{SiMe}_{3}\right)_{2}$ groups on tin are positioned above and below the plane defined by zirconium and the two tin atoms. The Sn-Zr distance is 287.15(11) pm and the $\mathrm{Sn}-\mathrm{Sn}$ distance of $423.64 \mathrm{pm}$ excludes a direct bond. Another dark red $\mathrm{Sn} 2 \mathrm{Zr}$ derivative [17] contains an approximately capped trigonal prismatic arrangement about zirconium, with one tin atom in a capping position. The mean $\mathrm{Sn}-\mathrm{Zr}$ distance of $301.2(3) \mathrm{pm}$ ia approximately $14.1 \mathrm{pm}$ longer than that in the previous example [16]. Each tin atom has pseudo-tetrahedral geometry $\left(\mathrm{SnC}_{2} \mathrm{Zr}_{2}\right)$. A black $\mathrm{Sn}_{2} \mathrm{Hf}$ derivative [18] is similar to that of the $\mathrm{Sn}-\mathrm{Zr}$ derivative [16] with a Sn-Hf bond distance of 295.3(1) pm and Sn-Hf-Sn angle of $81.14(3)^{\circ}$. Each of the metal atoms has a pseudo-tetrahedral geometry.

The structure of an orange $\mathrm{Sn}_{2} \mathrm{~V}$ cluster [19] consists of discrete tetraethylammonium cations and bis(triphenyltinpentacarbonylvanadate anions. The vanadium atom is heptacoordinated to the five carbonyl and two triphenyltin ligands as a monocapped trigonal prism. The Sn-V distances are 275.7(3) and 278.5(3) pm. with the Sn-V-Sn angle at $137.9(1)^{\circ}$. A red $\mathrm{Sn}_{2} \mathrm{Nb}$ derivative is similar to the $\mathrm{Sn} 2 \mathrm{Hf}$ derivative [18] with $\mathrm{Sn}-\mathrm{Nb}$ bond distances of $281.7(1)$ and $283.0(1) \mathrm{pm}$ and $\mathrm{Sn}-\mathrm{Nb}-\mathrm{Sn}$ angle of $87.18(3)^{\circ}$

There are four coloured $\mathrm{Sn}_{2} \mathrm{Cr}$ derivatives [21-23]. The first has a $\mathrm{Cr}(\mathrm{CO})_{4}$ moiety connected to two cisstannylene ligands with the $\mathrm{Sn}-\mathrm{Cr}-\mathrm{Sn}$ angle of $101.37(3)^{\circ}$ and mean $\mathrm{Sn}-\mathrm{Cr}$ bond distance of $255.7(1) \mathrm{pm}$. This 
distance is about $8.4 \mathrm{pm}$ shorter than that found in a gold yellow derivative [22a] at 264.1(1) pm with a Sn-CrSn angle of $127.49(4)^{\circ}$ which is about $26^{\circ}$ nore open than in the former case. Another yellow cluster has a central $\mathrm{Sn}$ atom bound by two $\mathrm{Cr}(\mathrm{CO})$ s moieties with $\mathrm{Sn}-\mathrm{Cr}$ bond distances of 271.6(2) and 275.5(2) pm. A quasitetrahedral environment about tin is completed by two terminal chlorine atoms $\left(\mathrm{SnCl}_{2} \mathrm{Cr}_{2}\right)$. A fourth derivative [23] has two $\mathrm{Me}_{3} \mathrm{Sn}$ moieties and a $\mathrm{Cr}(\mathrm{CO})_{s}$ unit held together by a $\mu_{3}-\mathrm{S}$ atom. This completes a tetrahedral geometry about each Sn(IV) atom and an octahedral geometry about the chromium atom (CrCsS). A yellow SnMo derivative [24] consists of two crystallographically independent molecules differing mostly by degree of distortion. The Mo atom is $\pi$-bonded to two cyclopentadienyl rings and through $\sigma$-bonds to the two Sn atoms (mean Sn-Mo bond distances of 272.3(1) and 272.5(1) pm). The Sn-Sn distances of 361.6(1) and 362.6(1) pm indicate the absence of any direct bonding. The structure of a Sn-W derivative [23] is found to be very similar to the $\mathrm{Sn}-\mathrm{Cr}$ counterpart discussed above [25].

There is one derivative [25] with $\mathrm{Sn}$ and $\mathrm{Mn}$ for which the structure is shown Figure 1. The complex contains both tri- and tetracoordinated tin atoms, trans across the manganese atom, with the stannyl group tin atom incorporated into a stannaindan ring system. The $\mathrm{Sn}-\mathrm{Mn}-\mathrm{Sn}$ bond angle is $168.2(2)^{\circ}$, and the Sn-Mn bond distances differ markedly at 251.3(2) and 270.0(2) pm indicating a double and a single bond, respectively. In a Sn-Re derivative [26] three units, $\mathrm{cp}(\mathrm{CO})_{2} \mathrm{Re}$ and two $\mathrm{SnPh}_{3}$, are connected by direct $\mathrm{Sn}$-Re bonds with a mean Sn-Re distance of $273.2(1) \mathrm{pm}$ and Sn-Re-Sn angle of $135.74(3)^{\circ}$.

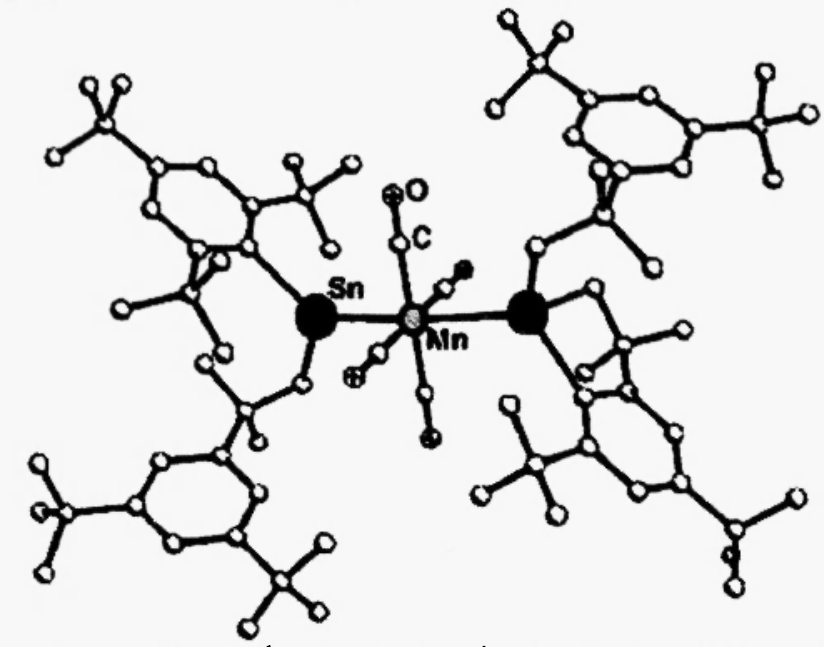

Fig. 1. Structure of $\left.\left[2,4,6-\mathrm{Bu}^{\prime} \mathrm{C}_{6} \mathrm{H}_{3}\right)\left\{3,5-\mathrm{Bu}_{2}^{\mathrm{t}} \mathrm{C}_{6} \mathrm{H}_{3} \mathrm{C}\left(\mathrm{Me}_{2}\right) \mathrm{CH}_{2}\right\} \mathrm{Sn}\right]_{2} \mathrm{Mn}(\mathrm{CO})_{4}[25]$

There are nine coloured $\mathrm{Sn}_{2} \mathrm{Fe}$ derivatives. The unit cell of $\mathrm{cp}(\mathrm{CO})(\mathrm{H}) \mathrm{Fe}\left(\mathrm{SnPh}_{3}\right)_{2}$ [27] contains two crystallographically independent molecules with essentially the same geometry. The structure can be described as a four legged piano stool, with the $\mathrm{SnPh}_{3}$ groups occupying the mutually trans basal positions. The mean $\mathrm{Sn}-\mathrm{Fe}$ bond distances of 256.391) and 256.691) pm are the shortest found in this set of derivatives and the Sn-Fe-Sn angle is the largest at $117.1^{\circ}$. Another three derivatives [28-30] have structures similar to that shown in Figure 1 [27]. An orange derivative has one $\mathrm{Ph}_{3} \mathrm{Sn}$ unit bound to one carbon atom of a $\eta^{3}$-indenyl group ( $\mathrm{Sn}-\mathrm{C}=$ 214.8(11) pm) which also serves as a bridge between the tin and a $\mathrm{Fe}(\mathrm{CO})_{2}$ unit. The second $\mathrm{Ph}_{3} \mathrm{Sn}$ unit is directly bonded to the $\mathrm{Fe}$ atom $\left(\mathrm{Sn}-\mathrm{Fe}=258.5(2) \mathrm{pm}\right.$. Both $\mathrm{Sn}$ atoms have tetrahedral geometry $\mathrm{SnC}_{4}$ and $\mathrm{SnC}_{3} \mathrm{Fe}$, respectively. Another orange derivative, $(\mathrm{Me} 2 \mathrm{Sn})_{2}(\mu-\mathrm{cp})_{2} \mathrm{Fe}$ [32] has almost parallel cyclopentadienyl rings (tilted $0.7^{\circ}$ towards the Sn-Sn pair) in a nearly eclipsed conformation (twist angle of $2.9^{\circ}$ ). The two Mes Sn units are displaced from the eclipsed arrangement by a torsion angle of about $5.3^{\circ}$. The Sn-Sn distance of 276.2(1) pm is the shortest in the $\mathrm{Sn}_{2} \mathrm{M}$ series of trimers, and each tin atom has a distorted tetrahedral geometry $\left(\mathrm{SnC}_{3} \mathrm{Sn}^{\prime}\right)$. A nearly linear array of nine atoms ( $\left.\mathrm{Cl}-\mathrm{Sn}-\mathrm{N}-\mathrm{C}-\mathrm{Fe}-\mathrm{C}-\mathrm{N}-\mathrm{Sn}-\mathrm{Cl}\right)$ with a total length of about $1,500 \mathrm{pm}$ is found in a yellow derivative [33]. A crystallographic inversion centre at the iron results from this linearity $\left(180^{\circ}\right)$ with the phenyl groups on the tin atoms arranged in a staggered geometry. The iron atom has essentially octahedral geometry (four $\mathrm{CN}$ carbon atoms and two dimethylsulphoxide sulphur atoms). The tin atoms have slightly distorted trigonal bipyramidal environments, with equatorial phenyl groups and a chlorine and a nitrogen atom occupying axial sites. A dark red derivative [34] has the Fe(II) atom encapsulated by the clatrochelate nioximate ligand and surrounded by a distorted trigonal antiprismatic coordination sphere of six nitrogen atoms from three dioxime units. The Sn(IV) atoms have slightly distorted octahedral geometry $\left(\mathrm{SnO}_{3} \mathrm{Cl}_{3}\right)$. The $\mathrm{Fe}(\mathrm{II})$ atom does not deviate much from the $\mathrm{Sn}$.. Sn axis $\left(\mathrm{Sn}-\mathrm{Fe}-\mathrm{Sn}=178.8^{\circ}\right)$. The $\mathrm{Sn}-\mathrm{Fe}$ distances at $361.2(1) \mathrm{pm}$ preclude a metal-metal bond. In a dark orange derivative $\left(\mathrm{Ph}_{3} \mathrm{Sn}\right)_{2}\left(\mu-\mathrm{Cs}_{5} \mathrm{H}_{4}\right)_{2} \mathrm{Fe}[35]$ the 
cyclopentadienyl rings of the ferrocene moiety are planar, with a dihedral angle of $1.4(3)^{\circ}$ between them. However, the presence of two bulky triphenyltin groups attached in a trans configuration causes the rings to be slightly staggered, the largest torsion angle being $19.4(4)^{\circ}$. The tin atoms with their bonded cyclopentadienyl rings are coplanar. The non-bonded Sn-Fe distances are 374.2 and $374.6 \mathrm{pm}$.

There are three yellow and one brown $\mathrm{Sn}_{2} \mathrm{Co}$ and derivatives [36-38]. In the latter [36] the central $\mathrm{Co}(\mathrm{CO})_{3}$ unit is bound to two $\mathrm{SnCl}_{3}$ units with $\mathrm{Co}-\mathrm{Sn}$ bond distances of 244.1(1) and 244.4(1) pm, respectively. Two crystallographically independent molecules are found in this system. The Sn-Co-Sn is almost linear, with an angle of $178.9(1)^{\circ}$ in one and $179.4(1)^{\circ}$ in the other. The tin atoms have distorted tetrahedral geometry $\left(\mathrm{SnCl}_{3} \mathrm{Co}\right)$ with the central cobalt in a trigonal bipyramidal arrangement $\left(\mathrm{Co}(\mathrm{CO})_{3} \mathrm{Sn}_{2}\right)$. The three yellow derivatives $[37,38]$ are isostructural with the $\mathrm{Sn} 2 \mathrm{Fe}$ derivative discussed above [34]. The Co atoms are encapsulated by the macrocyclic dioximate ligand and surrounded by a distorted trigonal antiprismatic coordination of six nitrogen donor atoms. Each Sn(IV) atom has a slightly distorted octahedral environment $\left(\mathrm{SnO}_{3} \mathrm{X}_{3}, \mathrm{X}=\mathrm{CI}\right.$ or $\left.\mathrm{Br}\right)$. The mean $\mathrm{Sn}-\mathrm{Co}$ distance is $360 \mathrm{pm}$ which does not indicate a metal-metal bond. The $\mathrm{Sn}-\mathrm{Co}$-Sn angle is very similar in all three complexes with a mean value of $179.5^{\circ}$.

The structure of a brown-black $\mathrm{Sn} 2 \mathrm{Ni}$ derivative [39] is shown in Figure 2. Cyclopentadienyl ligands are coordinated sandwich fashion to the three metal atoms. One cp ligand is $\pi$-bonded to the $\mathrm{Ni}$ atom such that the centre of the $\mathrm{cp}$ ligand and the three metal atoms lie almost within a plane. The second $\mathrm{cp}$ ligand symmetrically bridges the two stannylene units via the $\mathrm{Sn}$ atoms in a $\pi$-bonded manner with $\mathrm{Sn}$-C distances of $270.2(12)$ and $260.8(11) \mathrm{pm}$, respectively. In a blue derivative [40] two dithioxalate groups (tetradentate $\mathrm{O}_{2} \mathrm{~S}_{2}$ ) serve as bridges between the three metal atoms. The central $\mathrm{Ni}(\mathrm{II})$ atom is sulphur bonded (square planar $\mathrm{NiS}_{4}$ ) and the $\mathrm{Sn}(\mathrm{IV})$ are octahedrally coordinated $\left(\mathrm{SnO}_{2} \mathrm{Cl}_{4}\right)$.

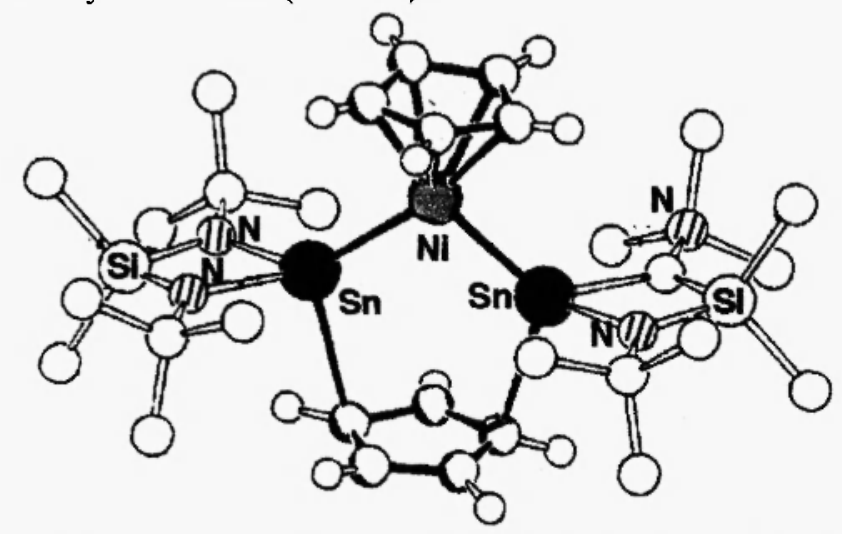

Fig.2. Structure of [cpNi\{Sn(NBu' $\left.\left.)_{2} \mathrm{SiMe}_{2}\right\}_{2}(\mu-\mathrm{cp})\right][39]$

A pink Sn-Ru derivative [41] has a slightly distorted octahedral geometry in which the $\mathrm{SnPh}_{3}$ units occupy axial positions about ruthenium $(\mathrm{Ru}-\mathrm{Sn}=268.6(2)$ and $269.1(2) \mathrm{pm})$. The $\mathrm{Sn}-\mathrm{Ru}-\mathrm{Sn}$ angle is nearly linear at $173.45(7)^{\circ}$ with a staggered conformation of phenyl groups. The $\mathrm{Ru}$ atom is six coordinate $\left(\mathrm{RuC}_{2} \mathrm{~N}_{2} \mathrm{Sn}_{2}\right)$.

A Sn-Rh complex [42] crystallises in a highly distorted square-planar cis geometry. The plane defined by $R h-P(1)-P(2)$ is twisted by $15.8^{\circ}$ relative to the plane defined by the three metal atoms. The complex may be considered as an insertion of a stannylene unit into the $\mathrm{Rh}-\mathrm{Cl}$ bond of the $\mathrm{RhCl}\left(\mathrm{PPh}_{3}\right)_{3}$ moiety. The most unusual feature of the structure is the chlorine bridged chelating bis(stannylene) ligand. This complex is somewhat reminiscent of the $\mathrm{Sn}_{2} \mathrm{Cl}_{s}$ complex anion. Both $\mathrm{Sn}-\mathrm{Rh}$ bond distances are almost equal (257.1(2) and 257.7(2) pm).

Yellow $\left(\mathrm{PMe}_{3}\right)_{2} \mathrm{Pd}\left(\mathrm{SnMe}_{3}\right)_{2}[43]$ has a twisted square planar structure. The distortion can be seen as a $16.8^{\circ}$ dihedral angle between the $\mathrm{PdP}_{2}$ and $\mathrm{PdS} \mathrm{n}_{2}$ planes. The $\mathrm{Sn}-\mathrm{Pd}$ bond distances are almost equal (260.4(1) and $260.7(1) \mathrm{pm}$ ) with the Sn-Pd-Sn angle at $80.71(3)^{\circ}$.

There are two $\mathrm{Sn}$-Os derivatives in this series, in both cases with six coordinate osmium atoms $[44,45]$. The $\mathrm{Sn}$ atoms are located at the apical positions and are tetrahedrally coordinated $\left(\mathrm{SnCl}_{3} \mathrm{O}[44]\right.$ and $\mathrm{SnC}_{3} \mathrm{O}_{3}$ [45]). The Sn-Pd distances are 264.8(1) and 271.7(1) pm, respectively, with an almost linear Sn-Os-Sn angle.

A red green derivative [46] contains complex anions with Snzlr and complex cations with Snlr2 frames, The complex anion has a trigonal bipyramidal iridium ( $\left.\mathrm{IrP}_{2} \mathrm{CSn}_{2}\right)$ with $\mathrm{Co}$ and $\mathrm{P}$ as the axial ligands (C-Ir- $\mathrm{P}$ angle $\left.=178.9(9)^{\circ}\right)$. The Sn-Ir bond distances are $256.9(2)^{\circ}$ and $259.2(3)^{\circ}$ with Sn-Ir-Sn angle of $108.7(2)^{\circ}$. The complex cation consists of the intact 12 atom metallocyclic ring (-IrPCAsCP-) 2 with the tin symmetrically 
bound to the two iridium atoms and a chlorine atom (Ir-Sn distances 274.1(2) pm, 274.2(2) pm: $\mathrm{Sn}-\mathrm{Cl}=$ $244.3(7) \mathrm{pm}$ and $\mathrm{Ir}-\mathrm{Sn}$-Ir angle $=146.1(1)^{\circ}$ in a planar arrangement.

There are seven Sn-Pt derivatives, one colourless and the rest yellow. Two of them, triclinic and monoclinic [47] contain cis- $\left[\mathrm{Cl}_{2} \mathrm{Pt}\left(\mathrm{SnCl}_{3}\right)_{2}\right]^{* 4}$ anions disordered in such a way as to be pseudo-centrosymmetric. This disordering involves half of the occupied $\mathrm{PtCl}_{2}$ sites appearing on either side of the centre. Simultaneously, one chlorine from each $\mathrm{SnCl}_{3}$ unit changes side while the other chlorine atoms appear in average positions with very small displacements between their positions. The Pt-Sn distance (235.56 pm ave.) is the shortest in the $\mathrm{Sn} 2 \mathrm{Pt}$ derivatives. The $\mathrm{Sn}-\mathrm{Pt}-\mathrm{Sn}$ angle is $111.48(3)^{\circ}$. Somewhat longer $\mathrm{Sn}-\mathrm{Pt}$ bonds $(248.9(1) \mathrm{pm})$ were found in another $c / s-\left[\mathrm{Cl}_{2} \mathrm{Pt}\left(\mathrm{SnCl}_{3}\right)_{2}\right]^{-2}$ anion with a smaller $\mathrm{Sn}-\mathrm{Pt}-\mathrm{Sn}$ angle of $97.8(2)^{\circ}{ }^{\text {[48]. Yellow }}$ $\left(\mathrm{PPh}_{3}\right)_{2} \mathrm{Pt}\left\{\mathrm{Sn}(\mathrm{acac})_{2}\right\}_{2}$ complex [49] has three metal moieties directly held by Sn-Pt bonds at an average distance of $255.8(1) \mathrm{pm}$. Square planar geometry is found in another complex, $\left\{\mathrm{P}(\mathrm{OPh})_{3}\right\}_{2} \mathrm{Pt}\left(\mathrm{SnCl}_{3}\right)_{2}[50]$ with $\mathrm{SnCl}_{3}$ ligands in trans positions. Each Sn(II) atom has a distorted tetrahedral geometry ( $\mathrm{SnCl} 3 \mathrm{Pt}$ ), and the $\mathrm{Sn}-\mathrm{Pt}$ bond distances are $258.8(2) \mathrm{pm}$. Another yellow complex [51] has three metal atoms bridged by two sulphur atoms in a 5 membered metallocyclic ring (-Pt-Sn-S-Sn-S-) with a Pt-Sn distance of 255.2(1) pm and Sn-S-Pt and Sn-S$\mathrm{Sn}$ angles of $108.2(1)^{\circ}$ and $100.1(1)^{\circ}$. The $\mathrm{Pt}(\mathrm{IV})$ atom has pseudo-octahedral arrangement $\left(\mathrm{PtN}_{2} \mathrm{C}_{2} \mathrm{SSn}\right)$ and each tin atom is tetrahedrally coordinated $\left(\mathrm{SnC}_{2} \mathrm{~S}_{2}\right.$ and $\left.\mathrm{SnC}_{2} \mathrm{SPt}\right)$. The colourless complex [52] also has a 5 membered metallocyclic ring (Pt-Sn-O-Sn-O-) with a Sn-Pt distance of $262.8(1) \mathrm{pm}$. All metals are four coordinate but each is different. The $\mathrm{Pt}$ atom is square planar $\left(\mathrm{PtP}_{2} \mathrm{OSn}\right)$, and the $\mathrm{Sn}$ atoms have tetrahedral geometry with different sets of donor atoms $\left(\mathrm{SnC}_{2} \mathrm{O}_{2}\right.$ and $\mathrm{SnC}_{2} \mathrm{OPt}$, respectively).

There are two $\mathrm{Sn}-\mathrm{Yb}$ complexes, dark orange [53] and yellow [54]. A noteworthy feature of the former is the pseudo-tetrahedral geometry about the $\mathrm{Yb}(\mathrm{II})$ atom $\left(\mathrm{YbO}_{2} \mathrm{Sn}\right)$, and the apparently long $\mathrm{Yb}$-Sn bond of $321.6(1) \mathrm{pm}$. Each tin is tetrahedrally coordinated $\left(\mathrm{SnC}_{3} \mathrm{Yb}\right)$ with a Sn- $\mathrm{Yb}-\mathrm{Sn}$ angle of $132.34(3)^{\circ}$. The other $\mathrm{Yb}$ complex [54] has a distorted octahedral structure. The $\mathrm{Yb}$ atom has four (thf)-O atoms in the equatorial plane and two $\mathrm{Ph} \mathrm{h}_{3} \mathrm{Sn}$ tin atoms in axial positions. The $\mathrm{Sn}-\mathrm{Yb}$ distance of $330.5(1) \mathrm{pm}$ is about $8.9 \mathrm{pm}$ longer than that of the orange derivative [53]. The $\mathrm{Sn}-\mathrm{Yb}-\mathrm{Sn}$ angle of $164.5(1)^{\circ}$ is larger than that of the orange case. Both tin atoms are again tetrahedrally coordinated $\left(\mathrm{SnC}_{3} \mathrm{Yb}\right)$.

\subsection{Sn plus Two $M$}

\subsubsection{Non-Transition Metal $M$}

A colourless lithium drivative [55] shows a complex structure in which six membered stannasiloxane rings are coplanar with each other and with four membered lithiostannoxane rings. The lithium atoms are tetrahedrally coordinated $\left(\mathrm{LiO}_{4}\right)$ and the tin atom is octahedrally configured $\left(\mathrm{SnO}_{4} \mathrm{Cl}_{2}\right)$. As indicated in Table 1 the Sn-Li distance is $301.3(11) \mathrm{pm}$.

The structure of an orange $\mathrm{SnCa}_{2}$ complex [56] has a three coordinated $\mathrm{Sn}(\mathrm{Il})$ atom $\left(\mathrm{SnP}_{3}\right)$ with six coordinated $\mathrm{Ca}(\mathrm{II})$ atoms $\left(\mathrm{CaO}_{3} \mathrm{P}_{3}\right.$ and $\mathrm{CaP}_{4} \mathrm{O}_{2}$ ). There are two crystallographically independent molecules present in the crystal. The average separation of $385 \mathrm{pm}$ between $\mathrm{Ca}$ atoms excludes a real metal-metal bond.

There is one $\mathrm{SnAl} 2$ derivative [57] which crystallizes in the centrosymmetric space group Pî The molecular structure consists of a central Sn(IV) atom with an octahedral arrangement of two terminal iodine atoms in cis positions, with four isopropoxy oxygen atoms of two bidentate $\left\{\mathrm{Al}\left(\mathrm{OPr}^{\prime}\right)_{4}\right\}^{\circ}$ anions.

\subsubsection{Transition or Lanthanide Metal $M$}

One $\mathrm{SnTi}_{2}$ derivative [57] crystallize in the acentric space group $\mathrm{P} 21$. In other respects the structure mirrors that of the $\left.\mathrm{SnA}\right|_{2}$ derivative mentioned above [57], except for the bidentate aluminium alkoxy anions which are now $\{\mathrm{Ti}(\mathrm{OPr})$ s $\}$ anions. Again, the Sn(IV) atom is in an octahedral arrangement of two iodine atoms and four oxygen atoms.

There is a red-orange and an orange $\mathrm{SnNb}_{2}$ derivative [58] both of which contain the $\mathrm{Nb}-\mathrm{Sn}-\mathrm{Nb}$ metal chain, with mean Nb-Sn bond distances of $284.0(2)$ and $286.2(1) \mathrm{pm}$. The angle at the tin atom is $140.69^{\circ}$ in one case and $129.44^{\circ}$ in the other (Table 1).

Four yellow and two orange derivatives are found with chromium as the heterometal atoms [59-62]. The four yellow derivatives $[59,60]$ contain the $\mathrm{Cr}-\mathrm{Sn}-\mathrm{Cr}$ metal chain with $\mathrm{Sn}-\mathrm{Cr}$ distances from $261.4(1)$ to 270.1(2) pm (average $266 \mathrm{pm}$ ). As the $\mathrm{Sn}$-Cr distance increases the $\mathrm{Cr}$-Sn-Cr angle closes. for example: $261.9(1)$ pm and $138.80(3)^{\circ}$ [59]; 264.2(4) pm and 131.6(1) ${ }^{\circ}$ [59]; 268.4(1) pm and 130.72(5) ${ }^{\circ}$ [59]: 269.7(2) pm and $130.2(1)^{\circ} \mathrm{pm}$ [60]. In each case the tin is in a tetrahedral environment. The remaining two orange complexes [61.62] contain a tin atom bonded to two methyl and two mesityl [61] or pheny [62] groups. Each mesityl or phenyl group is also bonded to $\mathrm{a} \operatorname{Cr}(\mathrm{CO})_{3}$ moiety in a $\eta^{6}$ fashion. The geometry around the tin atom is distorted tetrahedral $\left(\mathrm{SnC}_{4}\right)$.

A yellow [63] and an orange [57] SnMo2 cluster are isostructural with an Mo-Sn-Mo metal chain with a mean Mo-Sn distance of $300 \mathrm{pm}$ and Mo-Sn-Mo angle of $133.9(3)^{\circ}$. Each tin atom is tetrahedrally coordinated 
( $\mathrm{SnX}_{2} \mathrm{Mo}$, where $\mathrm{X}=\mathrm{Br}[63]$ or I [57]). The molybdenum atoms are also tetrahedrally coordinated ( $\mathrm{MocpC}_{3}$ ). There are four Sn-W derivatives [59,64-66]. The yellow-orange derivative has the W-Sn-W metal chain with Sn$W$ distances of $280.9(2) \mathrm{pm}$ and interbond angle of $130.62(4)^{\circ}$. The tin atom has a tetrahedral arrangement $\left(\mathrm{SnBr}_{2} \mathrm{~W}_{2}\right)$ and the tungsten atoms are pseudo-octahedral (WCs $\mathrm{Sn}_{5}$. The other $\mathrm{SnW}_{2}$ derivatives do not contain a metallic chain. Seven coordinate tungsten ( $4+3$ geometry) is found in another derivative. One tungsten is bonded to three bridging chlorine atoms and four $\mathrm{CO}$ groups, the other is bonded to three bridging chlorine atoms, three $\mathrm{CO}$ groups and the $\mathrm{Sn}$ atom of a $\mathrm{SnCl}$ moiety (a W-W-Sn arrangement). The W-Sn bond distance is $273.1(1) \mathrm{pm}$ and the two neighbouring tungsten atoms are $352.5(1) \mathrm{pm}$ apart. The tin atom has a distorted tetrahedral arrangement $\left(\mathrm{SnCl}_{3} W\right)$. The structure of a blue complex is shown Figure $3[65]$ and shows a $\mathrm{W}_{2} \mathrm{Sn}$ cluster with a (diethylamino)carbyne ligand bridging the tungsten atoms. The W-W axis is bridged by one $\left[\mathrm{S}_{2} \mathrm{CNEt}_{2}\right]^{-}$, one $[\mathrm{S}]^{-2}$ and the unusual dithiostannate(IV) ligand $\left[\mathrm{S}_{2} \mathrm{Sn}\left\{\mathrm{CH}(\mathrm{SiMe})_{2}\right\}_{2}\right]^{-2}$. A chelating dithiocarbamato ligand completes the roughly octahedral coordination environment around each tungsten (WSsC). A W-W bond distance of 261.4(2) pm and acute W-S-W angle of 69.3(2) and W-C-W angle of 77.5(6) are observed. The Sn(IV) atom has a tetrahedral environment $\mathrm{SnC}_{2} \mathrm{~S}_{2}$. An emerald complex [66] is observed to have a SnW 2 cluster with a sulphide and an alkyne bridge between the tungsten atoms. One of the tungsten atoms is linked to the tin atom by two sulphide bridges. The W(III)-W(IV) distance is $281.9(3) \mathrm{pm}$, the former being surrounded by four $\mathrm{S}$ and one $\mathrm{C}$ atom (WS4C), while the W(III) atom has three of each (WC $3 \mathrm{~S}_{3}$ ). The $\mathrm{Sn}(\mathrm{II})$ atom is tetrahedrally coordinated $\left(\mathrm{SnC}_{2} \mathrm{~S}_{2}\right)$.

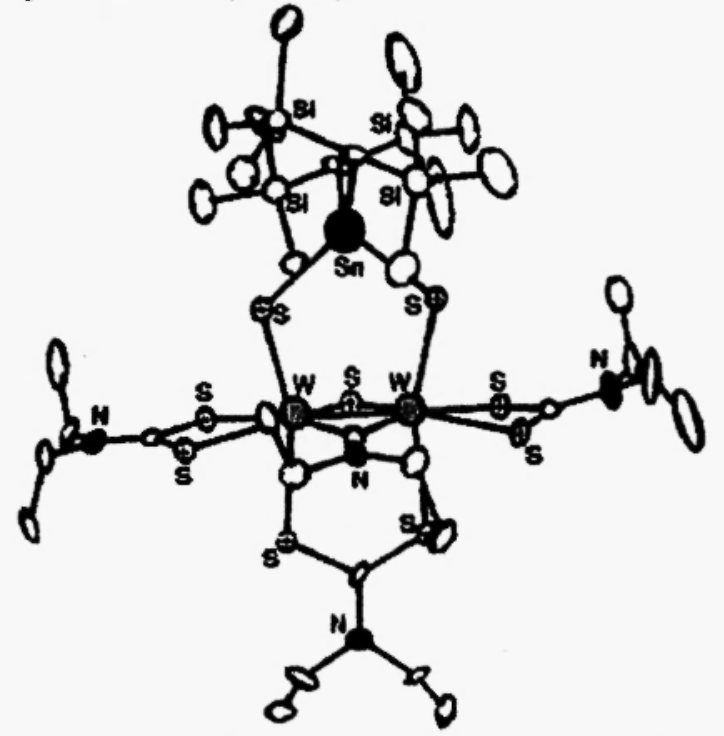

Fig.3. Structure of $\mathrm{W}_{2}(\text { dedtc })_{3}\left(\mathrm{CNEt}_{2}\right)(\mathrm{S})_{3} \mathrm{Sn}\left(\mathrm{CH}(\mathrm{SiMe})_{2}\right)_{2}$ [65]

There are ten derivatives with manganese as the heterometal atom [67-73]. Nine of these contain MnSn-Mn chains with Sn-Mn distances from 243.7(2) to 271.3(1) pm (average $254 \mathrm{pm}$ ). There is a trend for the $\mathrm{Sn}-\mathrm{Mn}$ bond distance to increase as the metal chain angle at tin closes: $245.8(2) \mathrm{pm}$ and $142.6(1)^{\circ}$ [67], 251.3(3) $\mathrm{pm}$ and $134.4(1)^{\circ}[70], 264.2(3) \mathrm{pm}$ and $125.88(9)^{\circ}$ [71], 271.2(1) pm and $121.9(1)^{\circ}$ [72]. Tin atoms in oxidation states of zero and +4 are observed. There are 3-coordinated $\mathrm{Sn}(\mathrm{O})$ examples $(\mathrm{SnXMn})$ with $\mathrm{X}=\mathrm{CI}$, $\mathrm{Br}$ or I [67], and 4-coodinated $\mathrm{Sn}(\mathrm{O})$ examples $\left(\mathrm{Sn}_{2} \mathrm{Mn}_{2}\right)[68,69]$. All of the $\mathrm{Sn}(\mathrm{IV})$ atom have tetrahedral geometry $\left(\mathrm{SnX}_{2} \mathrm{Mn}_{2}\right)$ where $\mathrm{X}=\mathrm{Cl}$ or $\mathrm{Br}$ [71] or phenyl [72]. For the 3-coordinate $\mathrm{Sn}(0)$ species the $\mathrm{Sn}-\mathrm{X}$ bond distance increases with covalent radius of $\mathrm{X}: 250.1(3) \mathrm{pm}(\mathrm{Cl}, 99 \mathrm{pm})<275.5(3) \mathrm{pm}(\mathrm{Br}, 114 \mathrm{pm})<298.5(1)$ $\mathrm{pm}(\mathrm{I}, 133 \mathrm{pm})$. At the same time the Sn-Mn bond distances also increases in the same order: $245.8(2) \mathrm{pm}<$ $246.4(3) \mathrm{pm}<246.8(2) \mathrm{pm}$. The same trends can be seen for the 4-coordinate Sn(IV) atoms with mean Sn(IV)-X and Sn-Mn bond distances of: 238.5(2) and 263.5(1) pm $(\mathrm{X}=\mathrm{CI})$ [71], and 254.8(2) and 264.2(3) pm $(\mathrm{X}=\mathrm{Br})$ [71]. Manganese atoms with $\mathrm{Sn}(\mathrm{O})$ atoms are all coordinated by cyclopentadienyl ring ligands plus two $\mathrm{CO}$ groups $\left(\mathrm{MnC}_{7}\right)$. Manganese atoms with $\mathrm{Sn}(\mathrm{IV})$ atoms are mostly bonded to five $\mathrm{CO}_{\text {groups }}\left(\mathrm{MnC}_{5}\right)$ except one study [72] where the manganese is bonded to four $\mathrm{CO}$ groups and one $\mathrm{MeNC}$ ligand $\left(\mathrm{MnC}_{5}\right)$. In the tenth

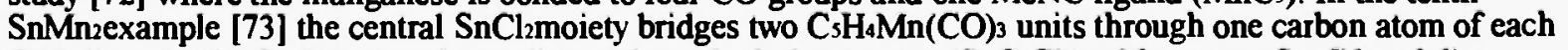
$\mathrm{C}_{3} \mathrm{H}_{4}$ ligand. The $\mathrm{Sn}(\mathrm{IV})$ atom has a distorted tetrahedral geometry $\left(\mathrm{SnC}_{2} \mathrm{Cl}_{2}\right)$ with a mean $\mathrm{Sn}-\mathrm{C}$ bond distance of $210.6(6) \mathrm{pm}$. Three derivatives have two Re atoms $[74,75]$. In two examples, colourless and dark red, a SnX 2 moiety bridges two $\operatorname{Re}(\mathrm{CO})_{4}\left(\mathrm{PPh}_{3}\right)$ units. The $\mathrm{Sn}-\mathrm{Re}$ bond distances are $273.0(2)$ and $274.1(2) \mathrm{pm}(\mathrm{X}=\mathrm{Br})$ and 
277.7(5) and 278. 1(4) pm $(\mathrm{X}=\mathrm{Cl})$. The corresponding Re-Sn-Re angles are $124.8(1)^{\circ}$ and $131.1(2)^{\circ}$. The Sn(IV) atom has a distorted tetrahedral configuration $\left(\mathrm{SnX}_{2} \mathrm{Re}_{2}\right)$. The third example is centrosymmetric $\operatorname{Sn}(\operatorname{tpp})\left\{\operatorname{Re}(\mathrm{CO})_{3}(\mathrm{C})\right\}_{2}$ [75] which contains two $\operatorname{Re}(\mathrm{CO})_{3}$ groups, one above and one below the plane of the porphyrin ring around the Sn(IV) atom. The tin and rhenium atoms are linked by a carbido $C$ atom. The Sn(IV) atom has a tetragonal bipyramidal arrangement with four tetraphenylporphyrinate $\mathrm{N}$ atoms in the plane and a carbon atom at the apical positions.

The iron containing $\mathrm{SnM}_{2}$ derivatives are the most abundant with sixteen examples [76-87]. Eleven of them [76-85] contain three metallic chains with Sn-Fe distances ranging from 249.0 to 265.0(4) pm (average 254 $\mathrm{pm}$ ) and Fe-Sn-Fe angles from $115.9(1)^{\circ}$ to $135.7(1)^{\circ}$ (average 126.3 ${ }^{\circ}$. There are another four derivatives [86] which are isostructural and contain a metal triangle with Sn-Fe bond distances from $260.3(2)$ to $261.6(21) \mathrm{pm}$ (average $261 \mathrm{pm}$ ) and $\mathrm{Fe}-\mathrm{Fe}$ bond distances from 278.892) to 279.7(2) pm (average $279.4 \mathrm{pm}$ ). The Fe-Sn-Fe ring angles have a small range of $64.6^{\circ}$ to $64.9(1)^{\circ}\left(64.7^{\circ}\right)$, but the $\mathrm{Fe}$ - Fe-Sn angles are about $7^{\circ}$ smaller $\left(57.6^{\circ}\right.$ to $58.0(1)$, average $57.7^{\circ}$. The most recent $\mathrm{SnFe}$ cluster [87] utilizes $\mathrm{SnCl}_{2}$ as a bridge between two $\left(\mathrm{C}_{5} \mathrm{H}_{4}\right) \mathrm{Fecp}$ moieties, with $\mathrm{Sn}-\mathrm{Cl}$ bond distances of $208.1(15) \mathrm{pm}$. The $\mathrm{Sn}-\mathrm{Fe}$ distance of $354 \mathrm{pm}$ precludes a direct metalmetal bond. In the sixteen clusters each $\mathrm{Sn}$ (IV) atom is tetrahedrally coordinated, $\mathrm{SnX}_{2} \mathrm{Fe}_{2}$ [76-86] and $\mathrm{SnCl}_{4}$ [87], but with differing degrees of distortion. The $\left\{\mathrm{Fe}(\mathrm{CO})_{4}\right\}_{2} \mathrm{Sn}\left(2,4,6-\mathrm{Pr}_{3}{ }_{3} \mathrm{C}_{6} \mathrm{H}_{2}\right)_{2}$ structure [87] contains two crystallographically independent clusters representing distortion isomers.

Eight derivatives contain $\mathrm{SnCo}_{2}$ clusters [88-94]. Five of them have a trimetallic $\mathrm{Co}-\mathrm{Sn}$-Co chain with Sn-Co bond distances from 249.891) to $257.5(8) \mathrm{pm}$ (average $253 \mathrm{pm}$ ) and Co-Sn-Co bond angles from $118.3^{\circ}$ to $129.3(1)^{\circ}$ (average $127^{\circ}$ ). A red derivative [92] contains a trimetallic ring with $\mathrm{Sn}$-Co bond distances of 256.4(3) and 259.1(3) pm and Co-Co bond distance of 262.694) pm. The Co-Sn-Co angle of 61.24(9) is larger than the $\mathrm{Sn}-\mathrm{Co}-\mathrm{Co}$ angles of $58.88(8)^{\circ}$ and $59.88(9)^{\circ}$. The two cobalt atoms are bridged by carbonyl group. In five of the clusters [88-91] the $\mathrm{Sn}(\mathrm{IV})$ atoms are tetrahedrally coordinated $\mathrm{SnX}_{2} \mathrm{CO}_{2}(\mathrm{X}=\mathrm{Cl}[88-91], \mathrm{X}=\mathrm{Ph}$ [88]) and in one case octahedrally coordinated $\mathrm{SnO}_{12} \mathrm{Cl}_{2}$ [92]. A light yellow cluster [93] has a central $\mathrm{Sn}(\mathrm{II})$ atom and two Cocp units at an angle, linked by two tripod ligands through $\mathrm{O}$ atoms to $\mathrm{Sn}(\mathrm{Il})$ and $\mathrm{P}$ atoms to Cocp. The $\mathrm{Sn}$ (II) atom has an octahedral environment $\mathrm{SnO}_{6}$ and the $\mathrm{Co}_{\text {atoms }}$ are in tetrahedral environments cpCoP. The Sn-Co distance of 437.2(8) pm (mean) is too large to consider as a metal-metal bond. Dark orange $\{\mathrm{cpCo}(\mathrm{db})\}_{2} \mathrm{Sn}[94\}$ contains two crystallographically independent molecules differing mostly by degree of distortion. Two virtually identical $\left.\mathrm{cpCO}_{2} \mathrm{C}_{2} \mathrm{~B}_{2} \mathrm{C}\right)$ sandwich units are bound to the central tin atom in a $\eta$ fashion via the bridging 1,3-biborolenyl ligands. The tetra-decker complex exhibits a bent structure in which the Sn-Co vectors form a $130^{\circ}$ angle.

A green $\mathrm{SnNi}_{2}$ cluster [95] contains a trimetallic ring (Ni-Sn-Ni) with $\mathrm{Sn}-\mathrm{Ni}$ bond distances of 246.591) and 246.9(1) pm, with Ni-Sn-Ni angle of 133.8(1).

Three derivatives [96-98] contain SnRuz clusters. A pale yellow one [96] has the three metals in a ring with Sn-Ru bond distances of 269.9(1) and 271.5(1) pm and a Ru-Ru bon distance of 295.4(2) pm. The Ru-Sn$\mathrm{Ru}$ angle of $66.1(1)^{\circ}$ is about $9^{\circ}$ more open than the $\mathrm{Sn}$-Ru-Ru angles of $56.7(1)^{\circ}$ and $57.2(1)^{\circ}$. The $\mathrm{Sn}$ atom is tetrahedral, $\mathrm{SnC}_{2} \mathrm{Ru}_{2}$. The structure of a derivative with a quadricyclic $\mathrm{SnS}_{2} \mathrm{Ru}_{2}$ ring is shown iFigure 4 [97]. The $\mathrm{Ru}(1)-\mathrm{Ru}(2)$ bond distance is $263.7(2) \mathrm{pm}$ and the $\mathrm{Ru}-\mathrm{S}-\mathrm{Ru}$ angle is $65.7^{\circ}$ compared to the mean $\mathrm{Ru}-\mathrm{S}-\mathrm{Sn}$ bond angle of $90.8^{\circ}$. In another derivative [98] the ruthenium atoms are both six coordinate, each bonded to three chlorine atoms on the outer side and two carbonyl groups plus a $\mathrm{SnCl}_{3}$ moiety on the inner side. The $\mathrm{Sn}-\mathrm{Ru}$ bond distance of $256.4(4) \mathrm{pm}$ is much shorter than that between the two ruthenium atoms (Ru-Ru distance $=$ 315.7(4) pm).

There are three $S n R h_{2}$ clusters $[99,100]$. In one of them [99] all three metals form a chain (Rh-Sn-Rh) with $\mathrm{Sn}-\mathrm{Rh}$ bond distances of 256.594$) \mathrm{pm}$ and two equal Rh-Sn-Rh bond angles of $143.52(3)^{\circ}$. The $\mathrm{Sn}$ (II) atom is pyramidally coordinated ( $\mathrm{SnCl}_{2} \mathrm{Rh}$ ) while each $\mathrm{Rh}(\mathrm{I})$ atom has a trigonal bipyramidal arrangement. $\mathrm{A}$ pale yellow SnRh2 cluster [100] has (cod) $\mathrm{Rh}^{+}$cations coordinated to two ethoxide ligands to produce a molecule with approximate $\mathrm{C}_{2}$ molecular symmetry. Of the six ethoxide ligands coordinated to tin, two cis-pairs are coordinated to $R h(1)$ and $R h(2)$, resulting in a distortion of the octahedral coordination environment for tin and distortion of the square-planar coordination environment of rhodium.

There is only one derivative with a SnPd2 cluster [101]. The X-ray analysis shows three metal atoms forming a nearly linear Pd-Pd-Sn chain (angle 165.8(1) $)^{\circ}$. The Sn-Pd and Pd-Pd distances are 258.592) and 264.4(2) pm, respectively. Two dppm ligands bridge two Pd atoms. The outer Pd atom is also coordinated by a chlorine atom to complete a distorted square planar arrangement ( $\left.\mathrm{PdP}_{2} \mathrm{ClPd}\right)$. The inner $\mathrm{Pd}$ atom also has a distorted square planar geometry ( $\left.\mathrm{PdP}_{2} \mathrm{SnPd}\right)$. The $\mathrm{Sn}(\mathrm{II})$ atom has tetrahedral geometry ( $\left.\mathrm{SnCl}_{3} \mathrm{Pd}\right)$.

A green Srlr2 cluster [102] consists of the intact metallomacrocycle (IrPC $4 \mathrm{P}_{2}$ with the $\mathrm{Sn}(\mathrm{II})$ atom bonded to it through the two Ir atoms. It has a crystallographically imposed $C_{2}$ symmetry with $C_{2}$ axis running through the tin atom and bisecting the $\mathrm{Cl}-\mathrm{Sn}-\mathrm{Cl}$ angle. The $\mathrm{Sn}$ (II) atom has a distorted tetrahedral environment $\left(\mathrm{SnCl}_{2} \mathrm{r}_{2}\right)$. The Sn-Ir bond distances are equal at 275.1(1) pm, and the Ir-Sn-Ir angle has the value 138.8(1) ${ }^{\circ}$. 
Finally, a dark ruby red cluster [103] contains two ytterbium atoms connected by three bridging phenyl rings. One of the $\mathrm{Yb}$ atoms is bonded to a triphenyltin moiety $(\mathrm{Sn}-\mathrm{Yb}=337.9(1) \mathrm{pm})$ and two tetrahydrofuran molecules. The second $\mathrm{Yb}$ atom is surrounded by three molecules of tetrahydrofuran, and the $\mathrm{Yb}-\mathrm{Yb}$ distance is $322.6(1) \mathrm{pm}$.

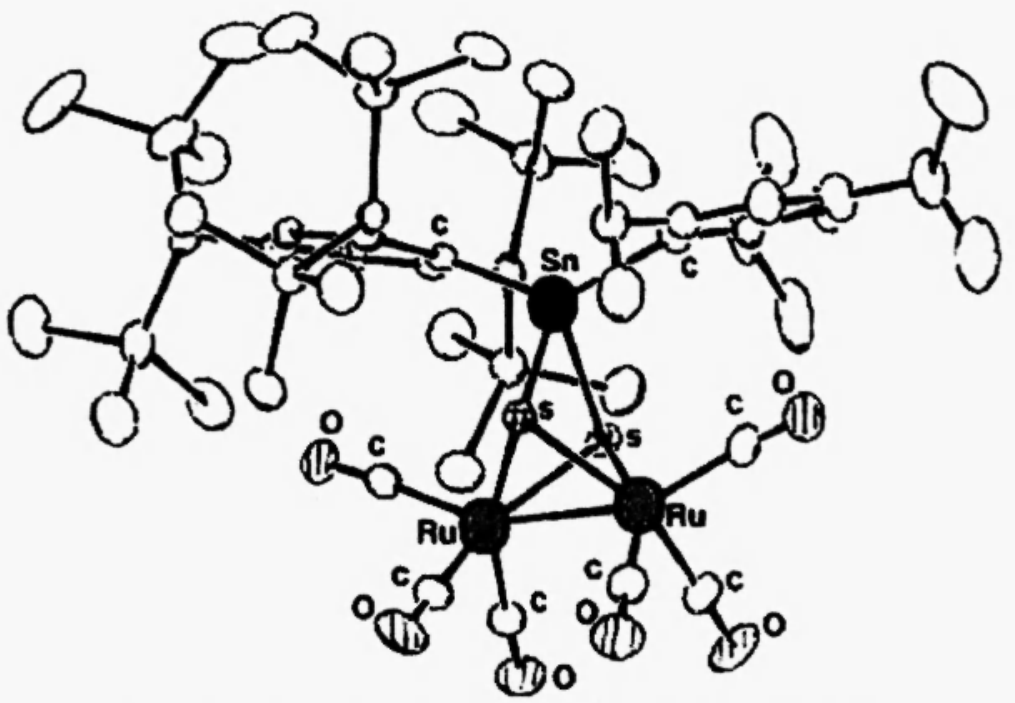

Fig. 4. Structure of (CO)6Ru2( $\mu-\mathrm{S})_{2} \mathrm{Sn}(\mathrm{tb})(\mathrm{tip})$ [97]

\subsection{Sn plus $M$ and $M^{\prime}$}

There are eight trimetallic derivatives that contain tin plus two different heterometal atoms. and their crystallographic and structural data are given in Table 1 . The first, an orange-red $\mathrm{cp}_{2} \mathrm{~W}(\mathrm{SnPh}) \mathrm{HgI}$ cluster [104], has a $\mathrm{cp}_{2} \mathrm{~W}$ unit directly bonded to $\mathrm{Hgl}(\mathrm{W}-\mathrm{Hg}=263.4(1) \mathrm{pm})$ and $\mathrm{SnPh}_{3}(\mathrm{~W}-\mathrm{Sn}=276.8(2) \mathrm{pm})$, The $\mathrm{Hg}-\mathrm{W}-\mathrm{Sn}$ angle is $78.87(6)^{\circ}$.

The yellow derivative $\left[\mathrm{MeGa}\left(\mu-\eta^{5}-\mathrm{pz}\right)_{3} \mathrm{Mo}(\mathrm{CO})_{3} \mathrm{SnPh}_{3}\right]$ cluster [105] has $\mathrm{MeGa}$ and $\mathrm{Mo}(\mathrm{CO})_{3}$ moieties connected by three $\eta$-pyrazolyl groups. The $\mathrm{Mo}(\mathrm{CO})_{3}$ unit is also connected to $\mathrm{SnPl}_{3}$ with a Mo-Sn bond distance of 285.79(3) pm. Both gallium and tin are tetrahedrally coordinated ( $\mathrm{GaN}_{3} \mathrm{C}$ and $\mathrm{SnC}_{3} \mathrm{Mo}$ ), and the central molybdenum atom is seven-coordinate $\left(\mathrm{MoN}_{3} \mathrm{C}_{3} \mathrm{Sn}\right)$. Another $\mathrm{Mo} / \mathrm{Sn}$ cluster includes thalium [106], this time with the tin atom in the centre. Three tert-butoxy groups bridge the $\mathrm{Sn}$ (II) and $\mathrm{Tl}(\mathrm{I})$ atoms, bringing them within 329.8(1) pm. In addition, a Mo(CO)s moiety is bonded to $\mathrm{Sn}$ (II) to complete 4-coordination with a SnMo bond distance of $277.0(2) \mathrm{pm}$. The Mo-Sn-Tl bond angle is almost linear at $178.8(1)^{\circ}$.

There are two derivatives with MnMoSn clusters [107,108]. The first is red and consists of $\mathrm{Mn}(\mathrm{CO})_{2}$ and $\mathrm{Mo}(\mathrm{CO})_{2} \mathrm{SnPh}_{3}$ units bridged by $\mathrm{S}_{2} \mathrm{CPcy}_{3}$ and trisisopropylphosphoniodithioformate ligands [107]. The Mn-Mo bond distance of 293.7(8) pm is about $13.8 \mathrm{pm}$ longer than the Mo-Sn distance (279.9(5) pm). The three metals have different stereochemistry. Tin is tetrahedrally coordinated $\left(\mathrm{SnC}_{3} \mathrm{Mo}\right)$, molybdenum is six coordinate $\left(\mathrm{MoC}_{2} \mathrm{~S}_{2} \mathrm{PMn}\right)$ and manganese is seven coordinate $\left(\mathrm{MnC}_{3} \mathrm{~S}_{2} \mathrm{PMo}\right)$, and the $\mathrm{Mn}-\mathrm{Mo}-\mathrm{Sn}$ angle is bent at $139.9(2)^{\circ}$. The second is yellow-orange and has $\mathrm{Mn}(\mathrm{CO})_{3}$ and $\mathrm{Mo}(\mathrm{CO})_{3}(\mathrm{SnPh} 3)$ units bridged by the $\mathrm{S}_{2} \mathrm{CPPr}^{i}$ ligand [108]. The Mn-Mo bond distance of $287.7(1) \mathrm{pm}$ is somewhat shorter than in the previous example [107], while the Mo-Sn bond distance of $281.0(1) \mathrm{pm}$ is about $1.1 \mathrm{pm}$ longer than previously. The stereochemistry about the atoms is similar to the other example [107], with the Mn-Mo-Sn angle about $5^{\circ}$ smaller at $134.9(2)^{\circ}$.

The structure of a pale yellow MnSnCo cluster [108] consists of a trimetallic chain with a tin atom in the middle. All the metal atoms are covalently bonded with distances of 272.8(10) pm (Sn-Mn) and 265.7(10) $\mathrm{pm}(\mathrm{Sn}-\mathrm{Co})$, and a Mn-Sn-Co bond angle of $114.4(2)^{\circ}$. The tin atom has a tetrahedral environment ( $\left.\mathrm{SnC}_{2} \mathrm{MnCo}\right)$ and the other two metals are octahedrally coordinated (MCsSn).

A deep red cluster [110] contains an almost linear Fe-Pd-Sn chain with bond distances of 266.55(6) $\mathrm{pm}$ (Fe-Pd), 259.50(5) pm (Pd-Sn) and an Fe-Pd-Sn bond angle of 169.34(2) ${ }^{\circ}$. The $\mathrm{Sn}$ and Pd atoms are four coordinate, tetrahedral $\mathrm{SnC}_{3} \mathrm{Pd}$ and square planar PdOPSnFe, while the $\mathrm{Fe}$ atom is six coordinate (FeC2PSiPd). A bright yellow cluster [111] has a trimetallic chain of Fe-Sn-Pt atoms. The bond distances are 256.8(2) pm (Sn-Fe) and 252.4(2) pm (Sn-Pt), with an Fe-Sn-Pt bond angle of 115.8(1). The Pt and Fe atoms are bridged by a dppe ligand giving a six membered metallocyclic ring. The tin atom is tetrahedrally coordinated 


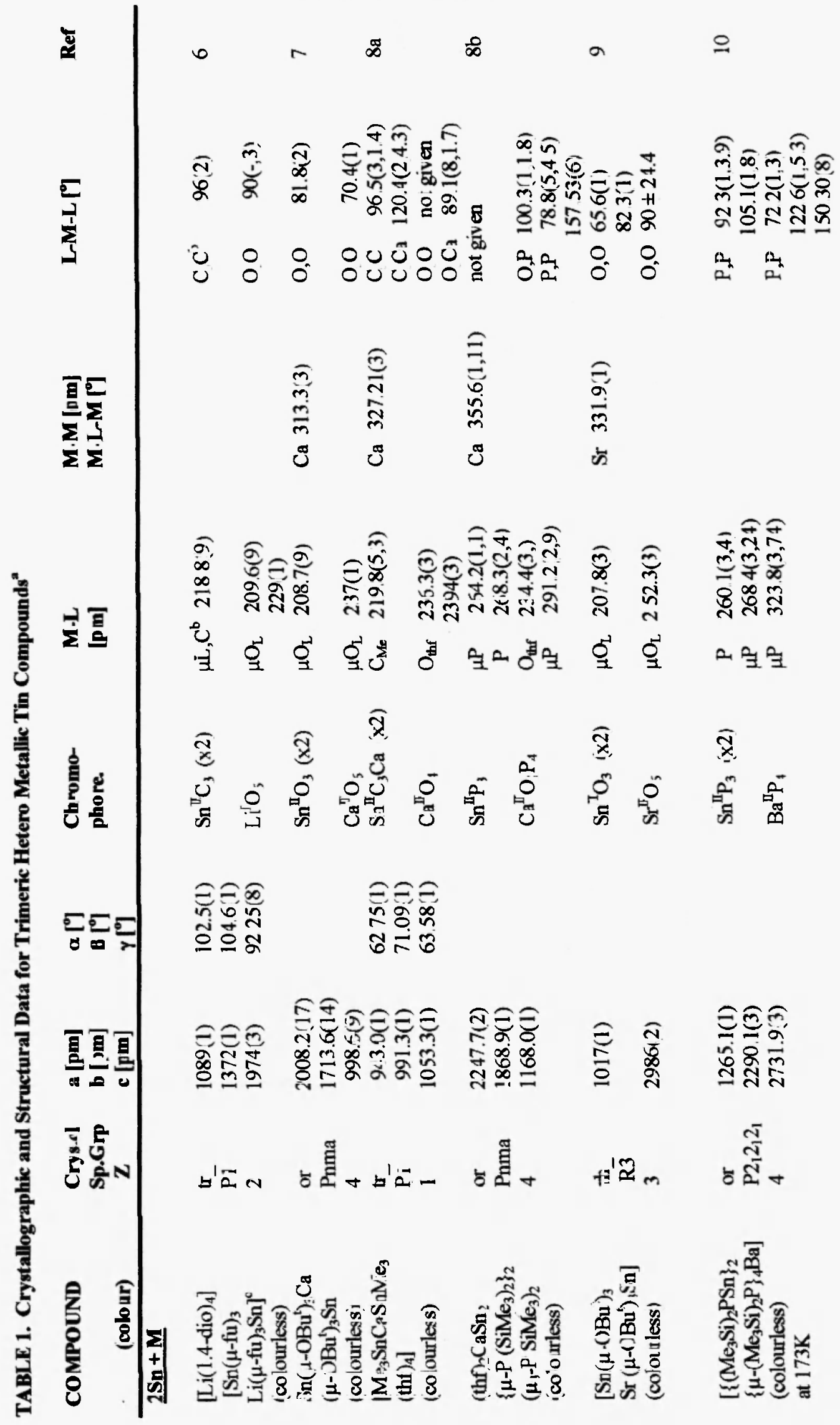


으

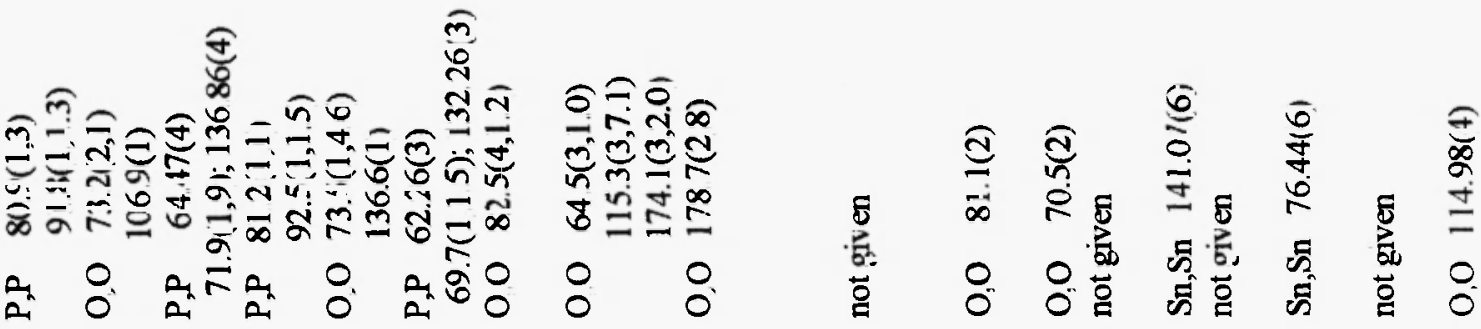

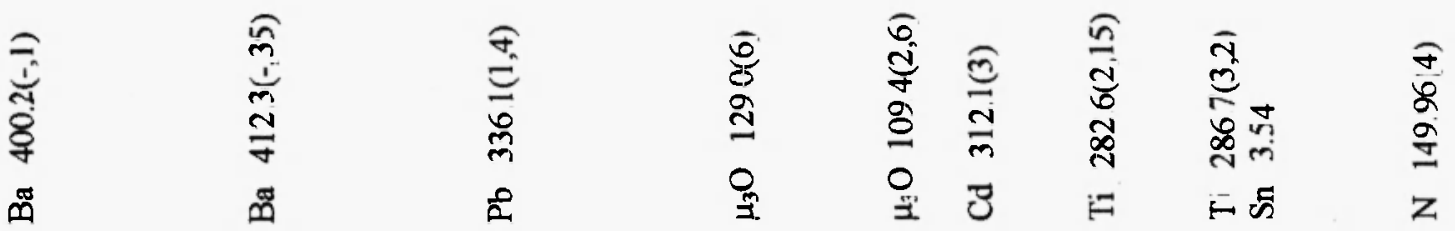

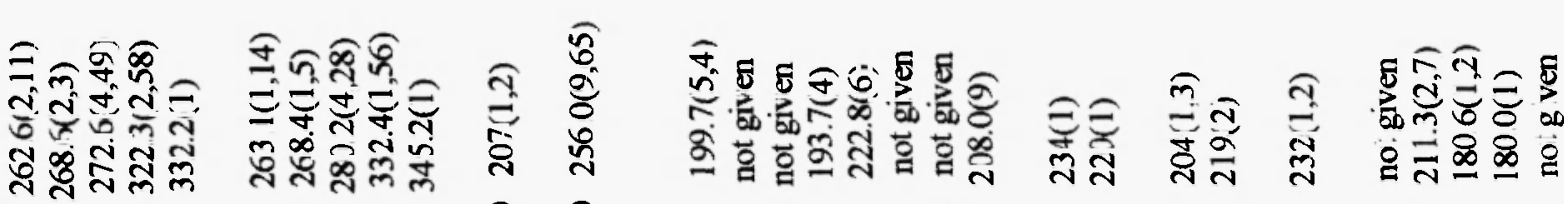

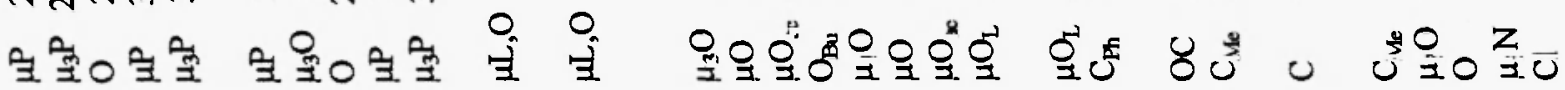

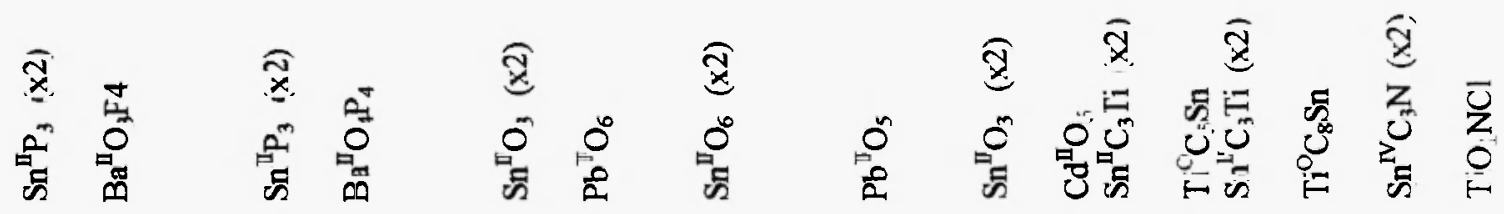

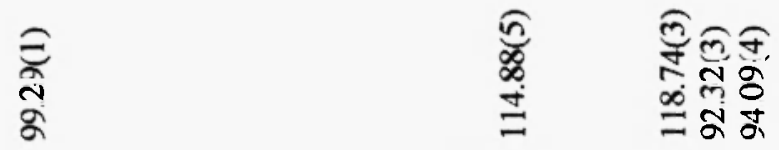

ลิตฺำ

त

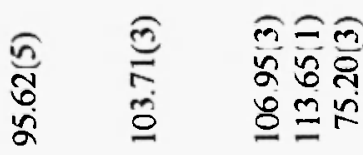

드응

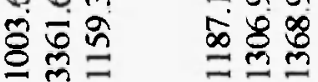

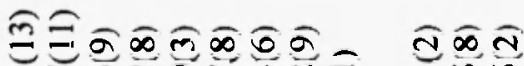

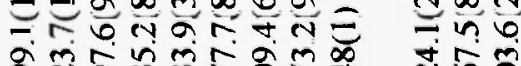

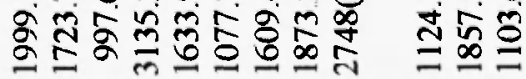

$\Xi \stackrel{0}{\bar{c}} \infty$

E气⿱艹

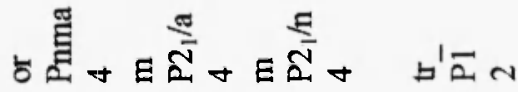

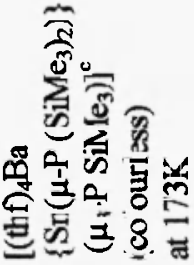

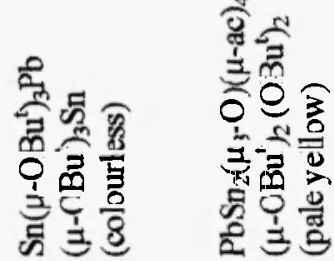

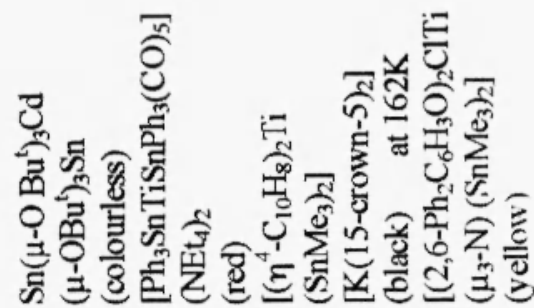


$\because$

$\stackrel{0}{2}$

$\therefore \quad \infty$

$\sqrt[4]{2}$

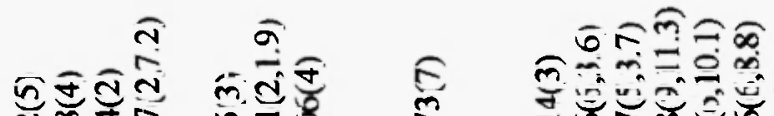

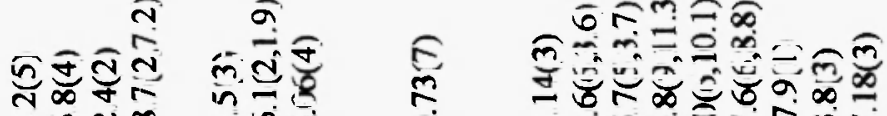

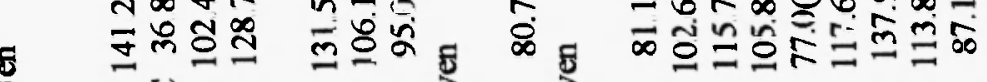

ชิ่ง

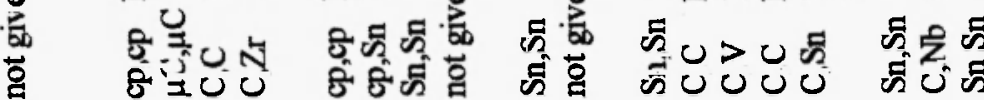

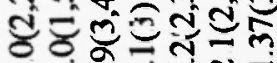

กำ

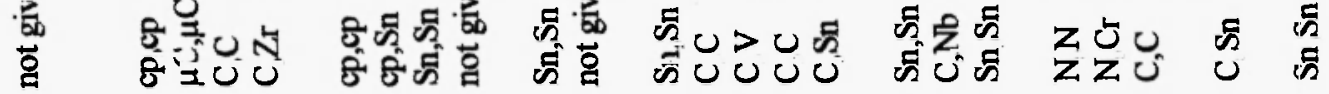

\begin{tabular}{|c|c|c|c|c|}
\hline 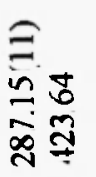 & 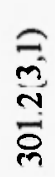 & 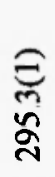 & 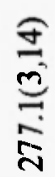 & 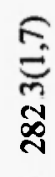 \\
\hline 노 & 노 & 江 & $>$ & 之 \\
\hline
\end{tabular}

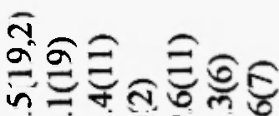

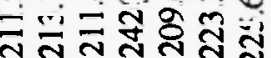

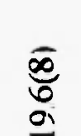

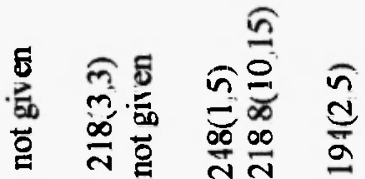

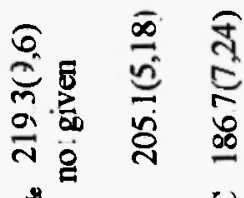

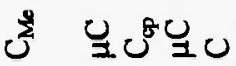

లँ

ปे z

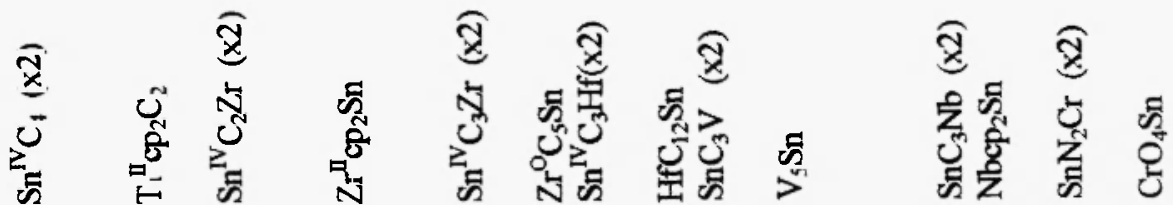
$\frac{\nwarrow}{8}$
$\frac{n}{6}$
$\begin{array}{ll}\stackrel{\Xi}{\Xi} & \Xi \\ \Xi & \sigma\end{array}$
$\underset{\Xi}{\infty}$
$\vdots$
5

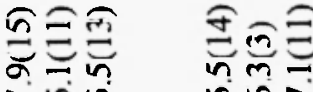

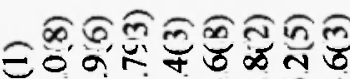

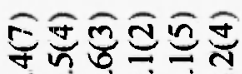

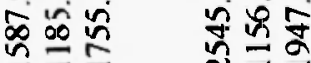

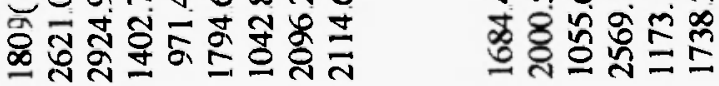

घ

น

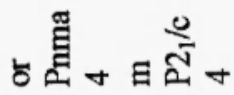
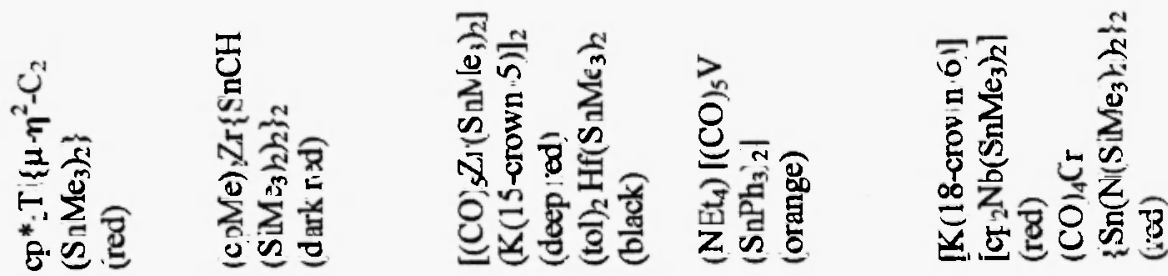
สี

สิก

$\vec{n}$

สิ สิ

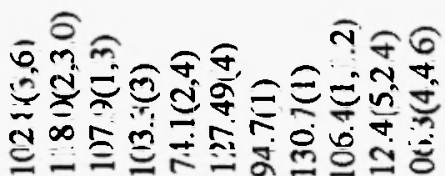

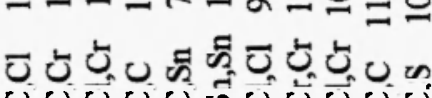

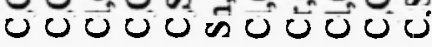

0
$\substack{0 \\ 0 \\ 0 \\ 0}$
0

高

$\stackrel{\overbrace{}}{\stackrel{0}{*}}$

,

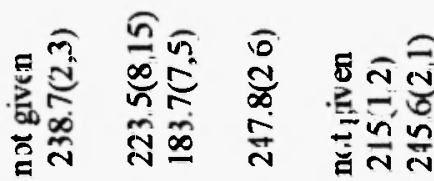

Uี่

$\widehat{\widehat{x}}$

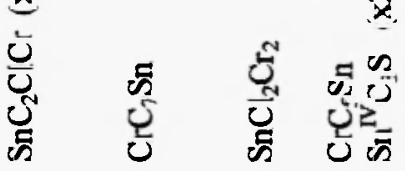

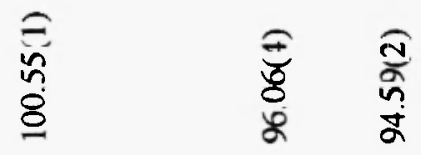

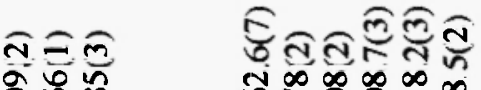

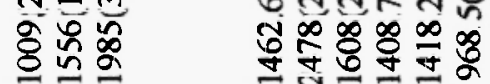

a若+

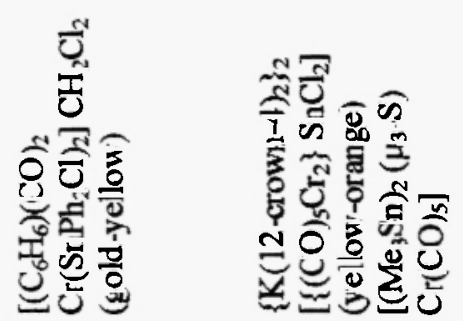

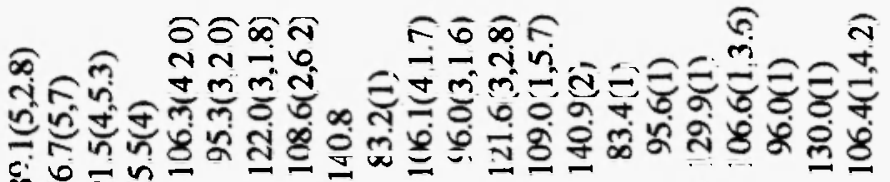
क⿺辶万人

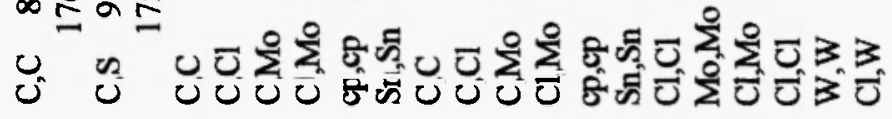

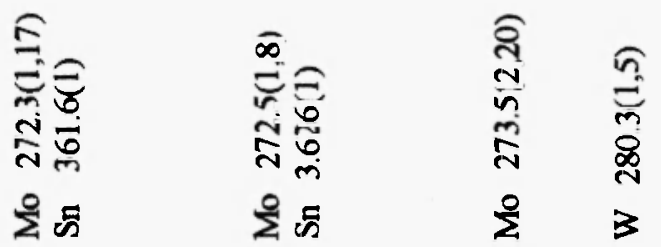

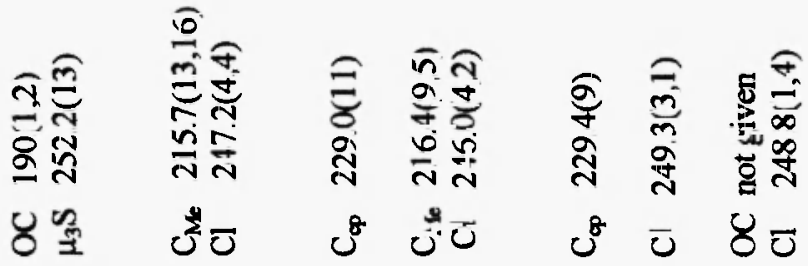

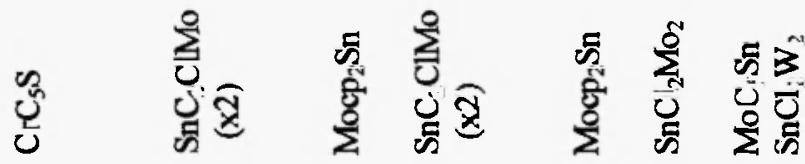

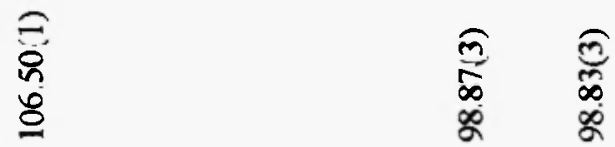

ฮิฐ్ల్

$\overline{\underline{0}}=\overline{\underline{y}} \overline{\underline{0}}=\overline{\mathrm{g}}$

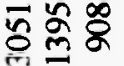

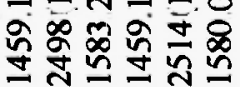

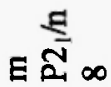

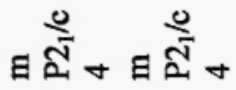

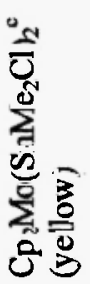

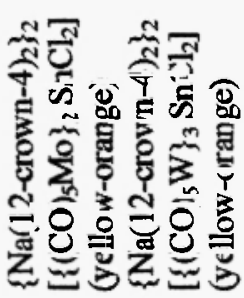


3

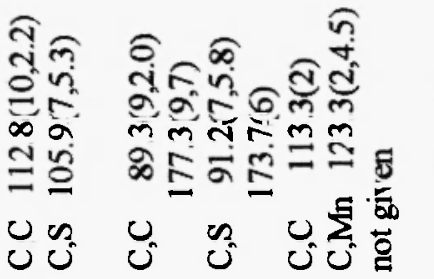

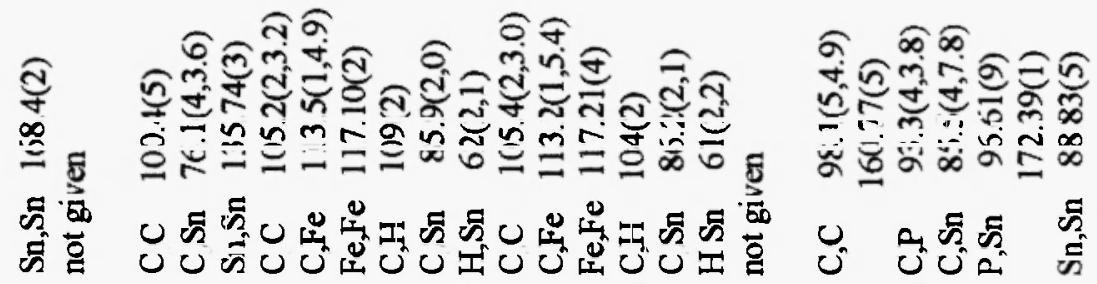

\section{로ำ}

ஸ்

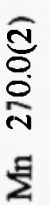

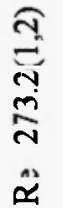<smiles>C1=C=CC=CC=1</smiles>

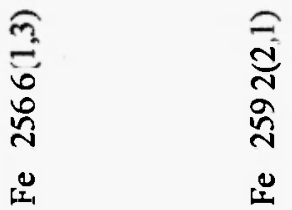

ปิ

$\exists$ 옹 웡요

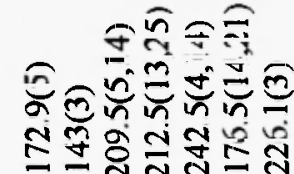
㑩<smiles>C#CC#CC#C</smiles>

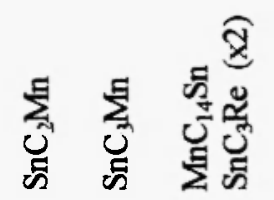

$\widehat{\widetilde{x}}$
0
$\tilde{y}$
$\tilde{n}$

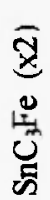

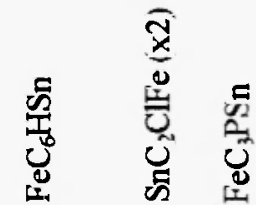

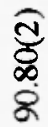

ถิก

ลิ ฐิ

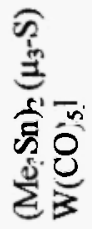

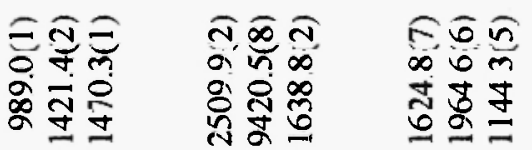

เ゙๋ナ

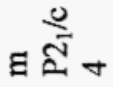

E $\stackrel{\Sigma}{E}+$<smiles>C1C[Te]C[Te]1</smiles>

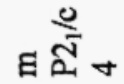

$$
\text { bั十 }
$$
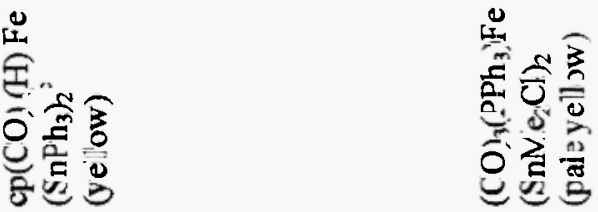
ลे

요

$\bar{m}$

స్

$m$

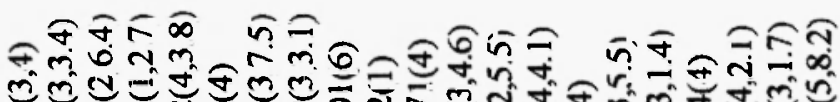

क

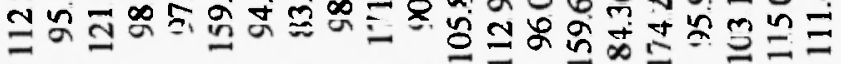

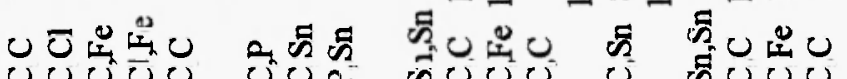

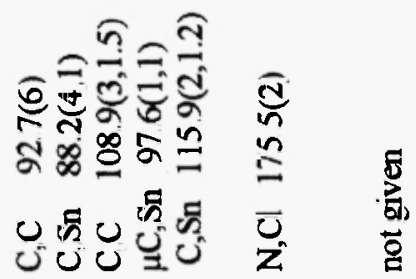

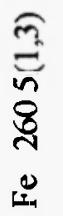

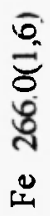

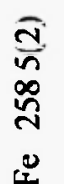

ते
टे
हี

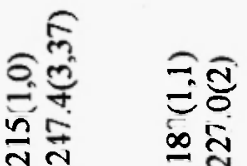

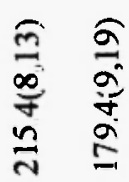

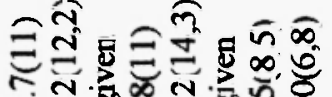

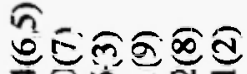

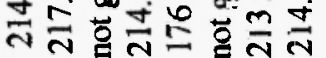

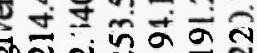

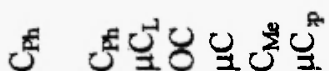

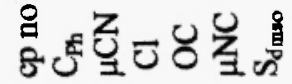

ปี兀

ธี

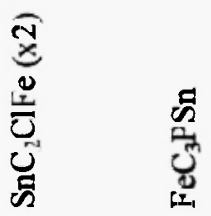

$\widehat{x}$

总氧

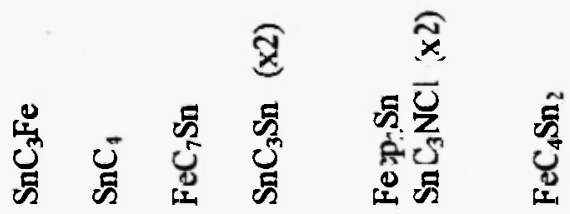

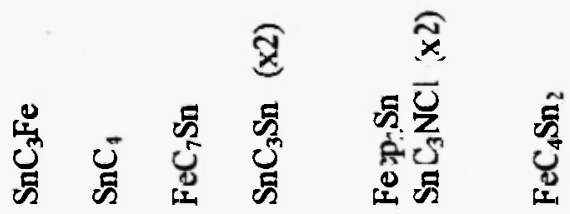

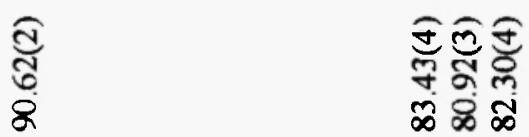

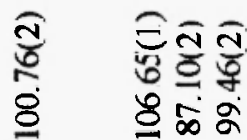

సิำ

可语

흥ㅎㅇ

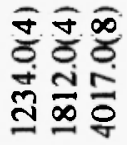

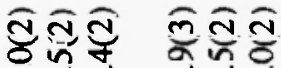

$\stackrel{\text { 至 }}{=}$

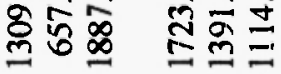

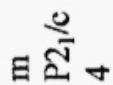

घ๐

苾葛

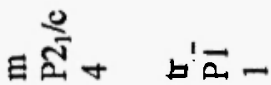
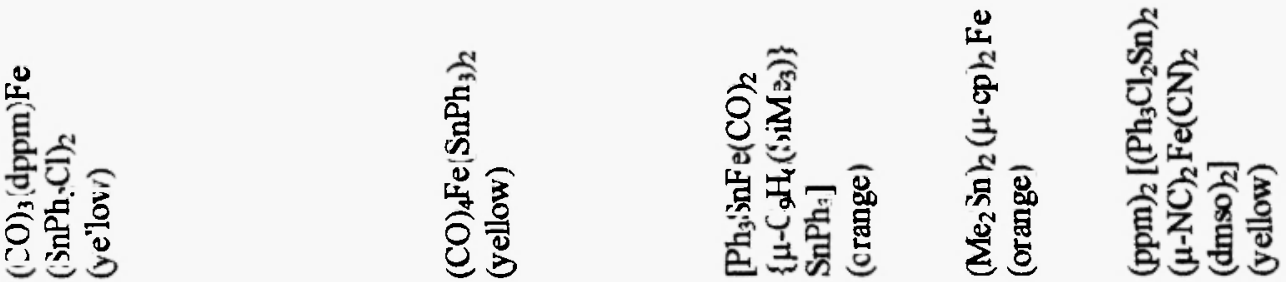
m

$\infty$

$\stackrel{\infty}{m}$

i

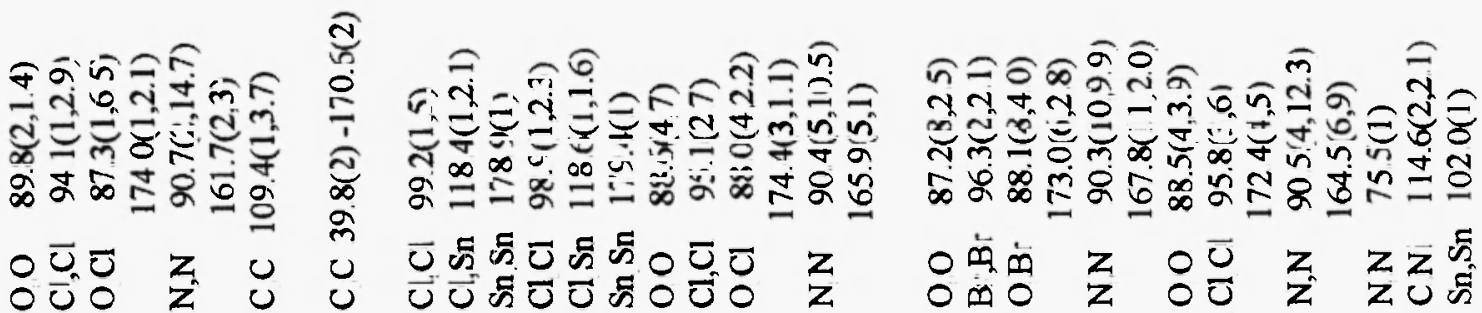

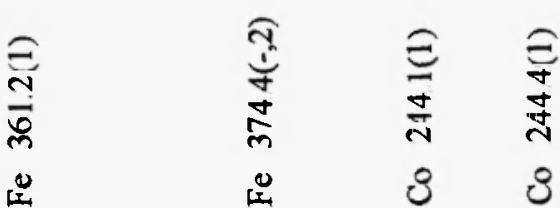

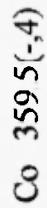

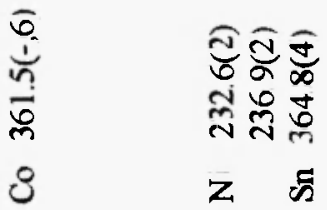



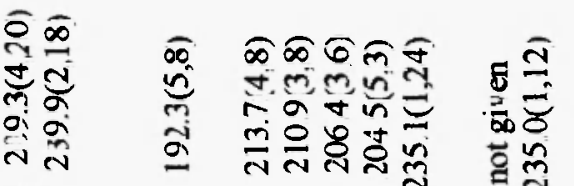

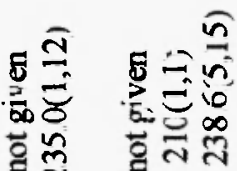

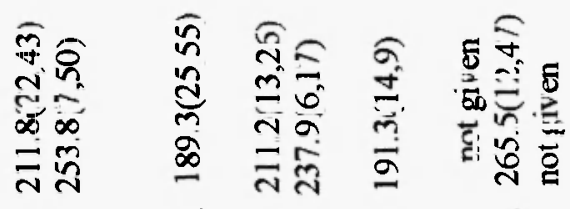
主च

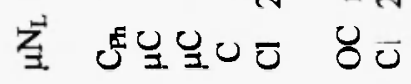

8

$\stackrel{\circ}{\circ}$

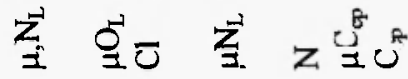

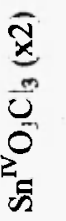

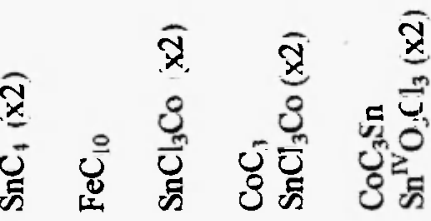

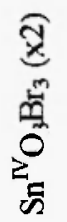

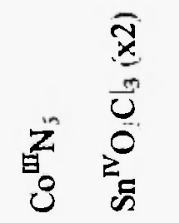

$\bar{x}$

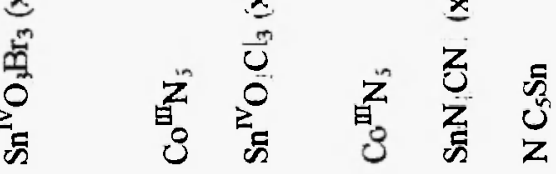

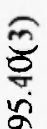

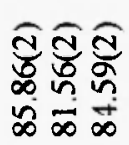

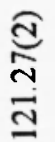

$\underset{\infty}{\varrho}$

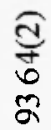

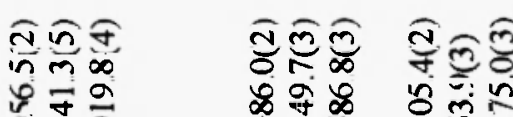

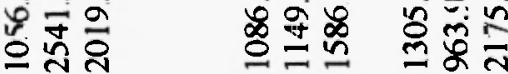

을

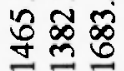

으ㅇㅝㅠ

产绾㐫

枽守

菓然

웅

吉象造

$\frac{a^{2}}{a^{2}}+$

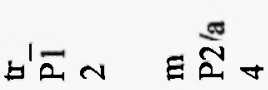

ฐ ัญ

ธูป ำ

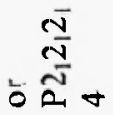

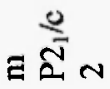
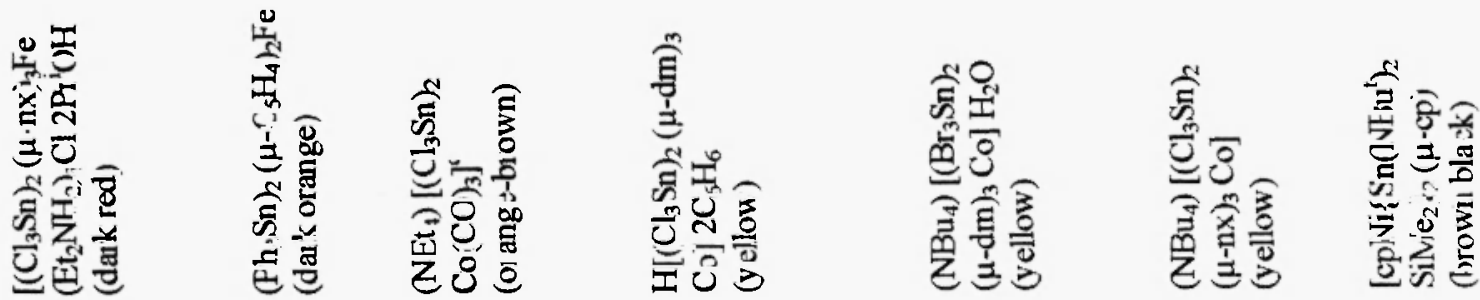
q $\bar{\nabla}$

ช

$q$

$\exists$

q

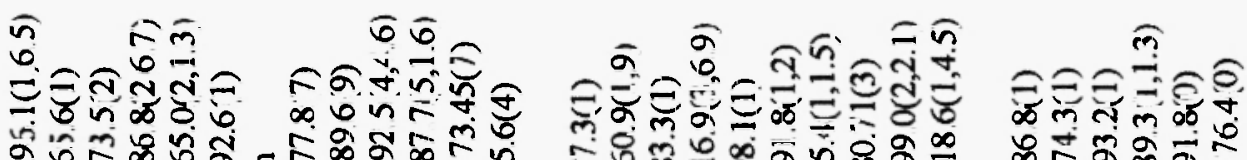

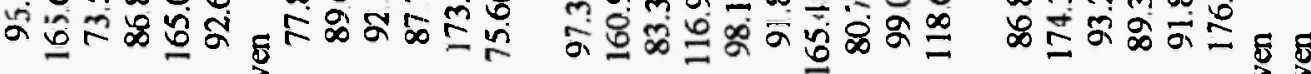

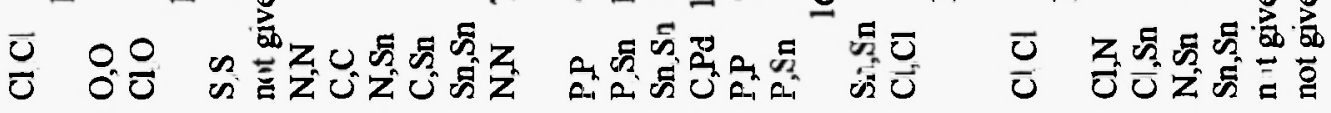

\begin{tabular}{|c|c|c|c|}
\hline 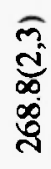 & $\begin{array}{l}\bar{a} \\
\stackrel{\mathbb{J}}{a} \\
\tilde{n}\end{array}$ & 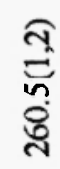 & 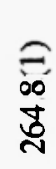 \\
\hline $\bar{\alpha}$ & 모 & $\overrightarrow{0}$ & 8 \\
\hline
\end{tabular}

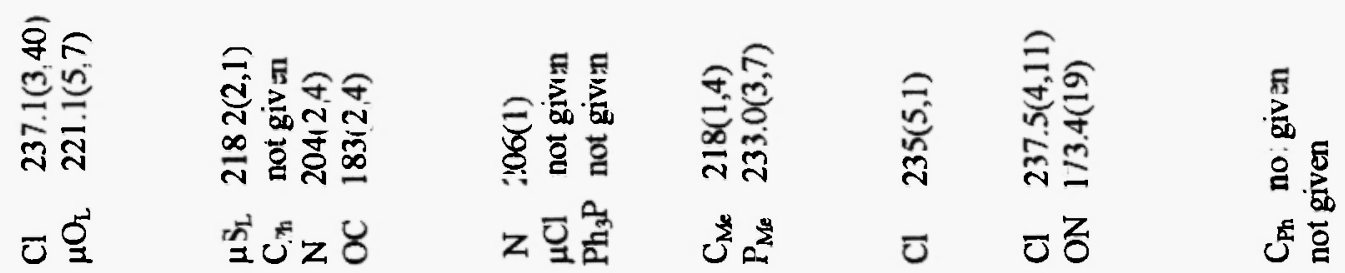

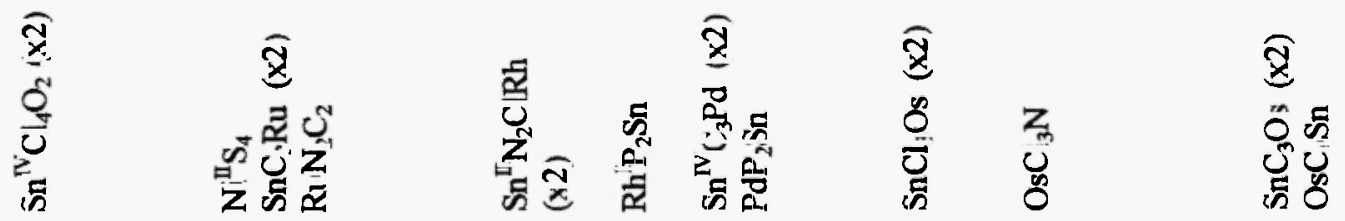

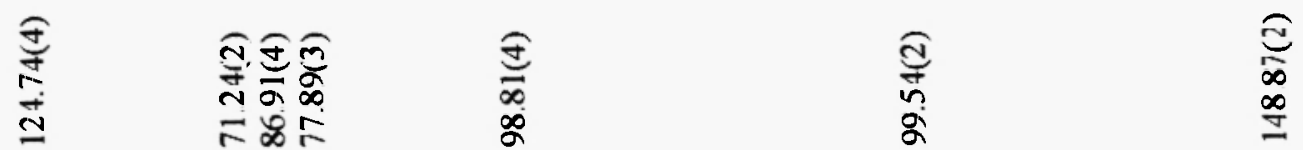

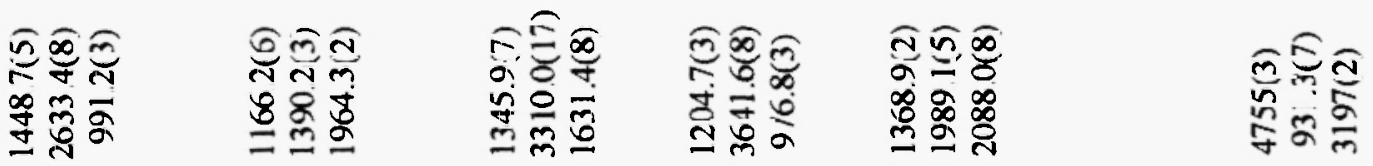

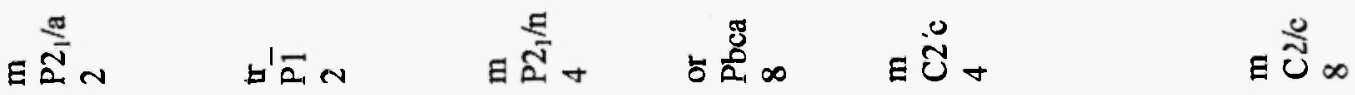

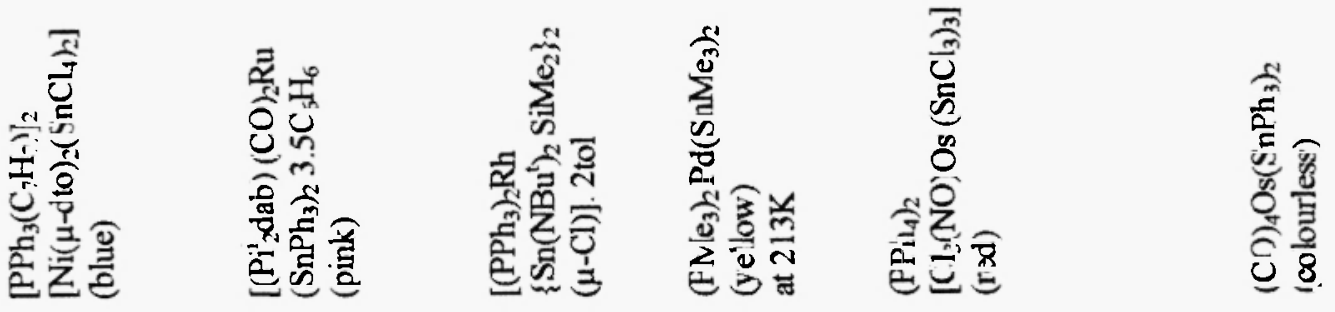


$\because$

F

\& $\quad \stackrel{\infty}{\square}$

q

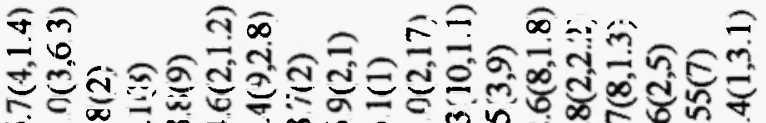

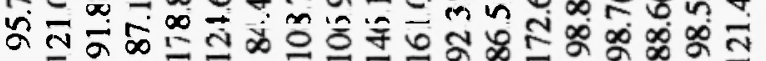

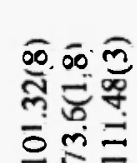

ลุ่

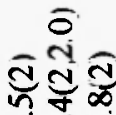

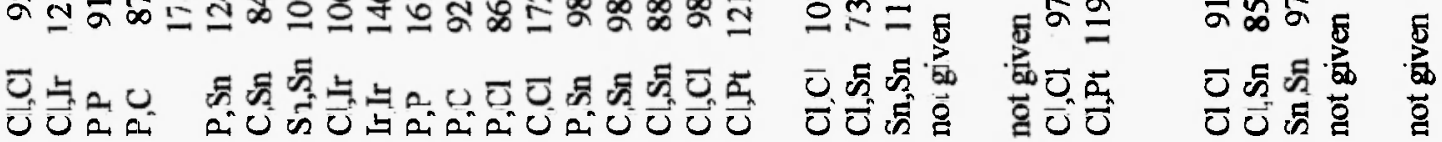

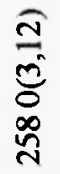

上

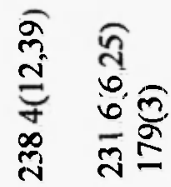

บ $2 \varnothing$

$\widehat{x}$

氖 矛

ลิ

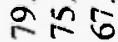

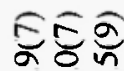

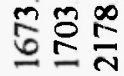

๒ยล

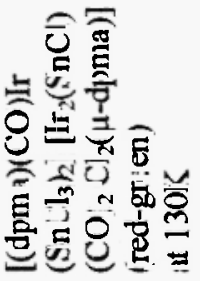

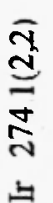

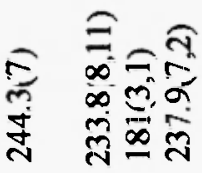

च

ब্்

苞

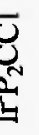

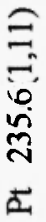

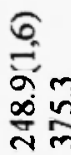

立荕

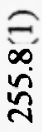

a

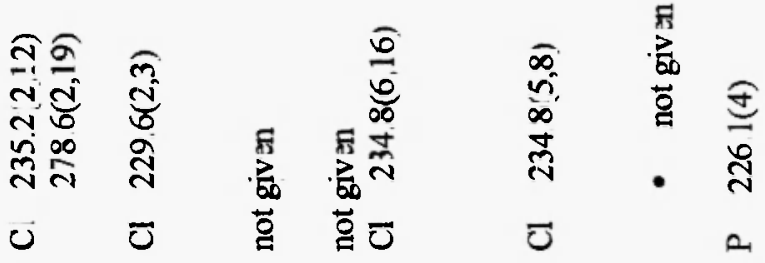

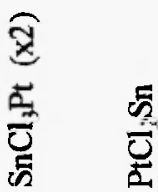

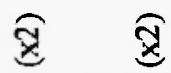

$\widehat{x}$

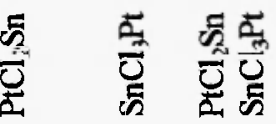

芯 㫄

즈르.

m.

응

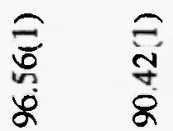

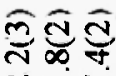

동ํㅇ

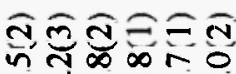

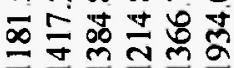

$\widehat{ } \widehat{\widetilde{v}}$

๙ั่

ط

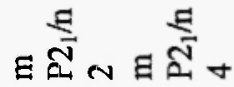

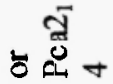
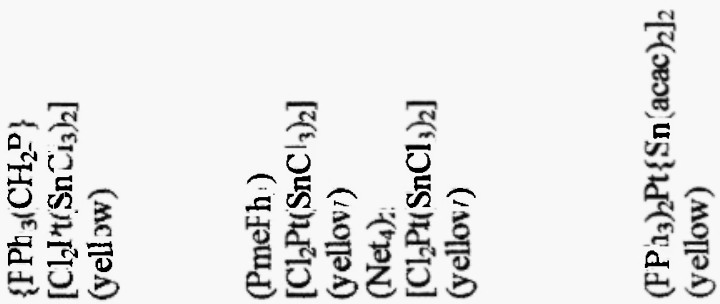
\& $\bar{n}$

ถี

$n$

in

ิิต

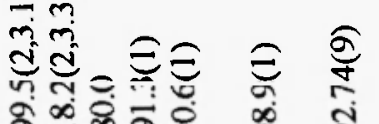

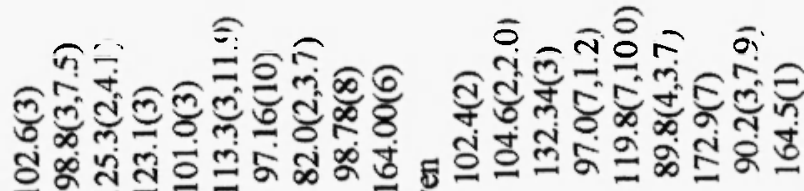

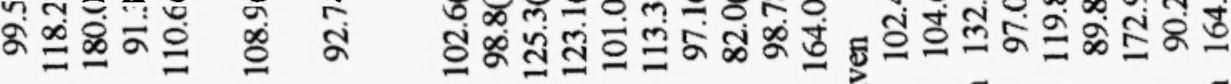

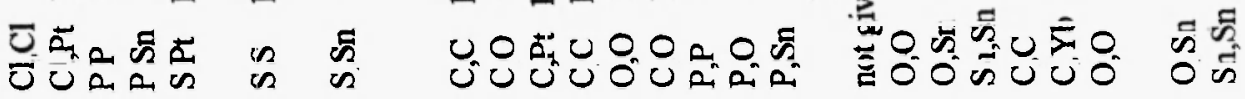

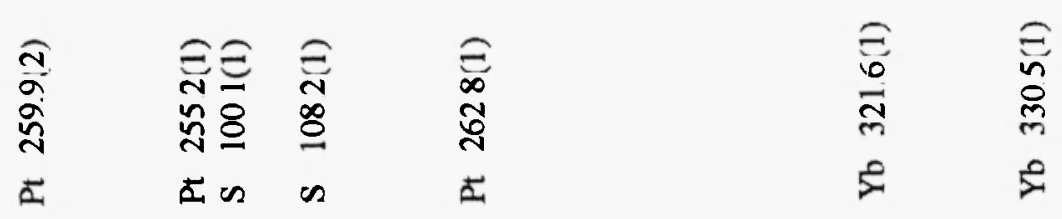

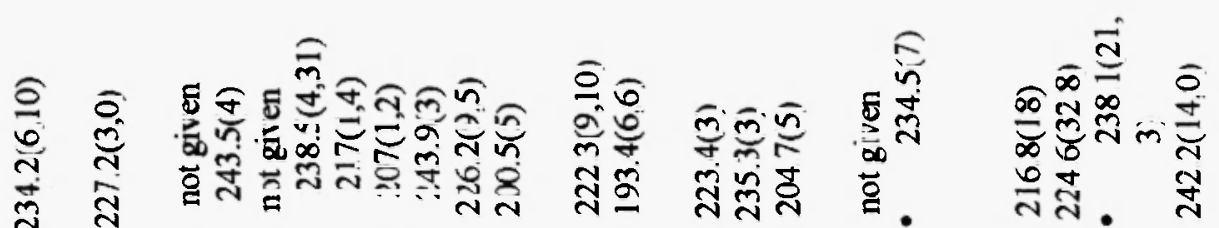

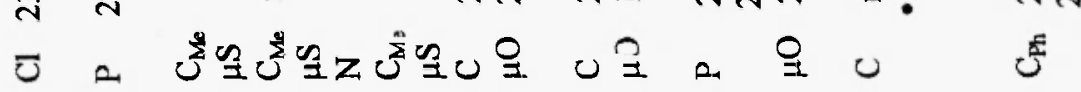

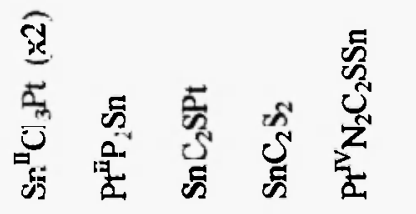

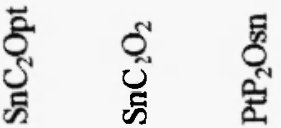

শิ $\widehat{x}$

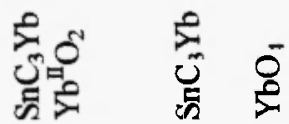

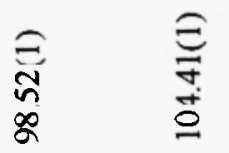

อิำ

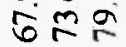

$\begin{array}{ll}\stackrel{0}{5} & \hat{a} \\ \stackrel{0}{a} & \frac{2}{2}\end{array}$

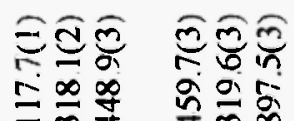

ิำ

ชิ

守点旁

क्षक

봉ㅎㅀ

\&

$\vec{b}^{\prime} \vec{\alpha} N$

घ

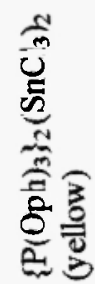

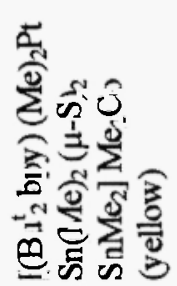

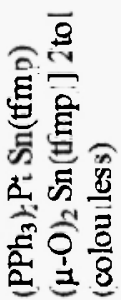

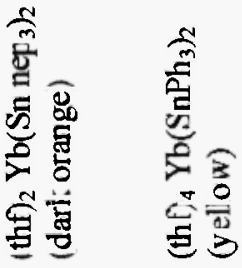


in

$\wp$

in

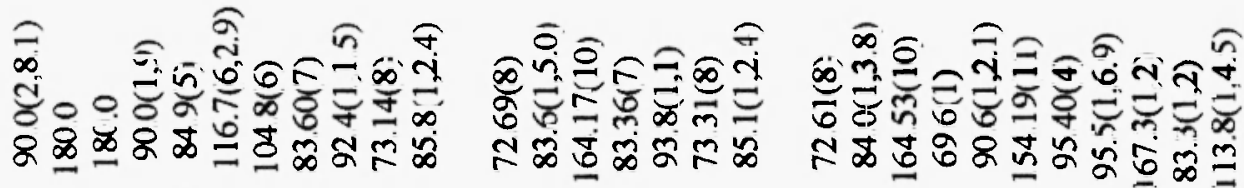

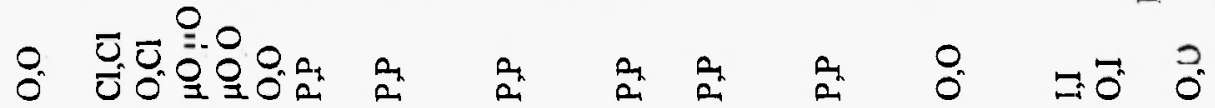

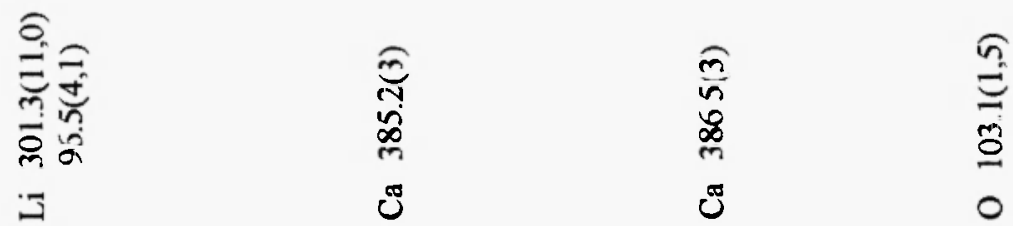

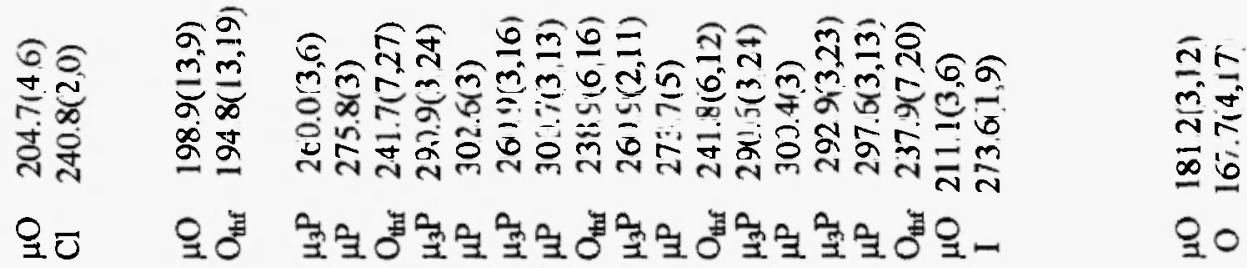

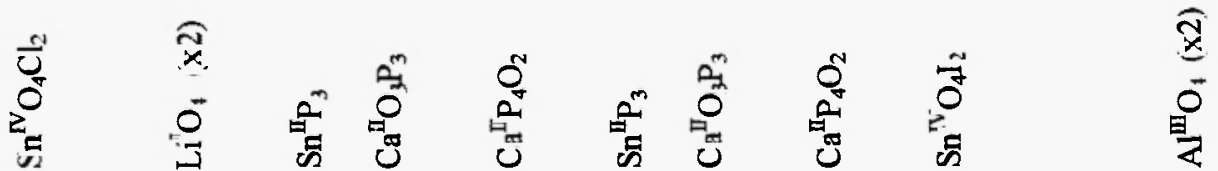

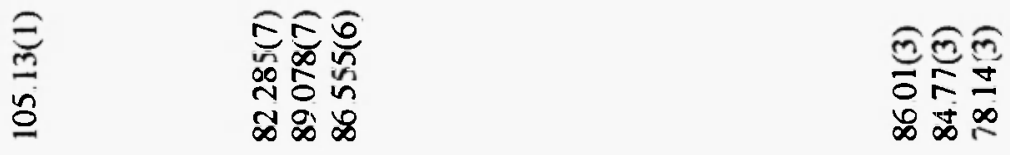

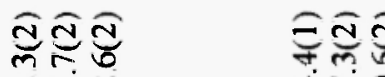

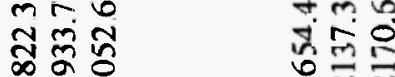

可은

สิติติ

$\bar{n} \infty 0$

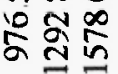

E $\stackrel{\pi}{E} N$

$b^{\prime} \overline{a_{1}} N$
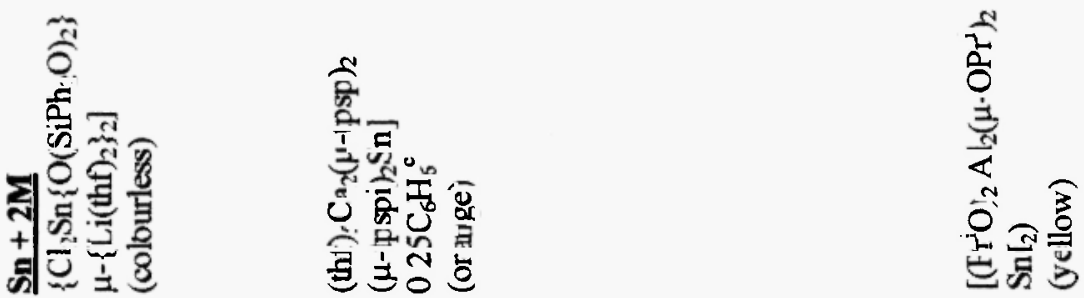
in

$\infty$

in

in

के

众

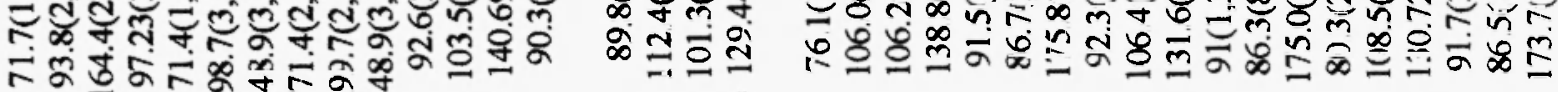

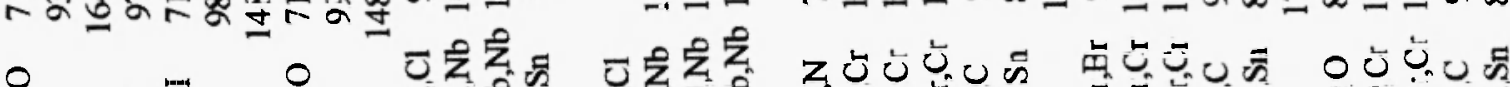
○ चல

\begin{tabular}{|c|c|c|c|c|}
\hline तิ & 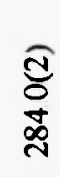 & 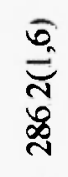 & 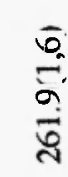 & $\begin{array}{l}\text { Tे } \\
\text { ț } \\
\text { ț }\end{array}$ \\
\hline & $\mathbf{z}$ & zे & Ü & $\dot{u}$ \\
\hline
\end{tabular}

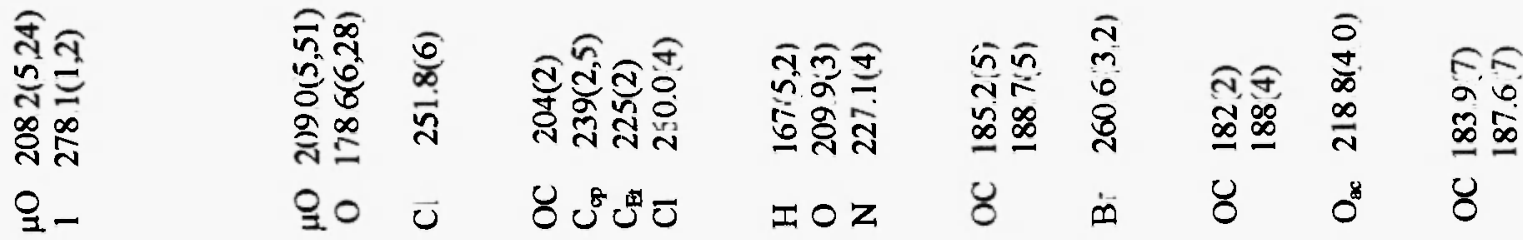

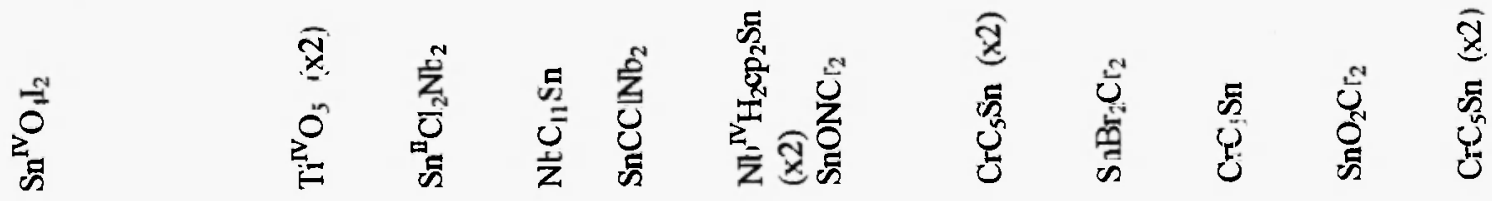
?
๙ิ్
$\underset{\vartheta}{\stackrel{\vartheta}{\Xi}}$

ลิำต

กำ

을 응

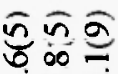

宇象

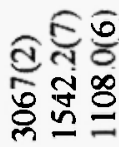

$\infty \pi \frac{n}{\infty}$

동용

$E \bar{\Sigma}$

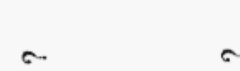

घ $\frac{\text { ลे }}{4}$

ธ

घ ักั
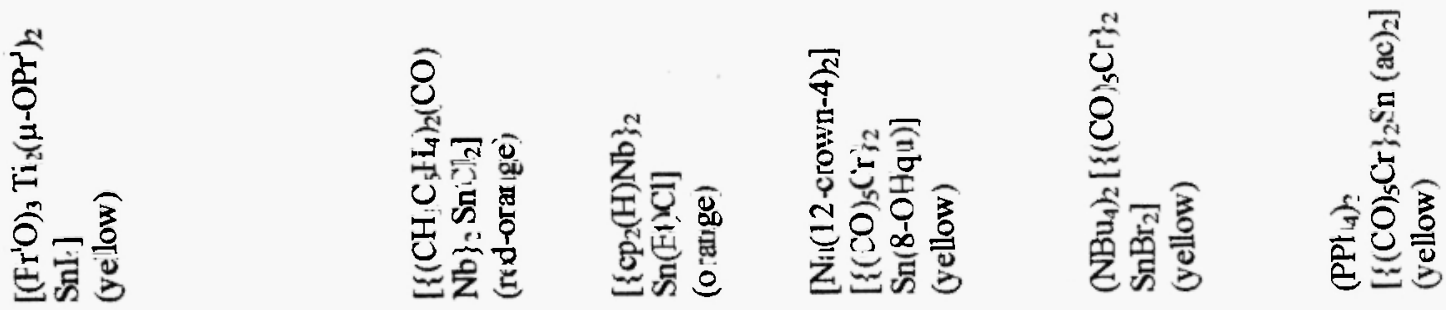
8

$\overline{6}$

ธี

$\beta \quad$ in

के

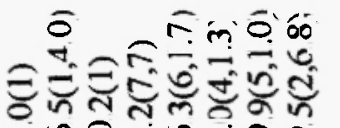

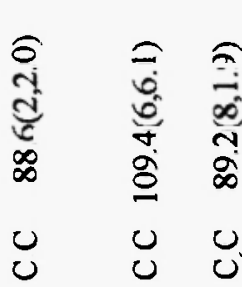

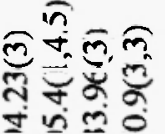

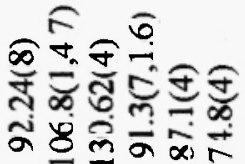

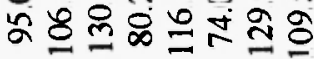

包邑只

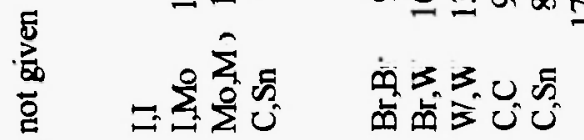

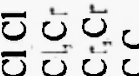

है 0

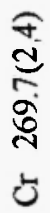

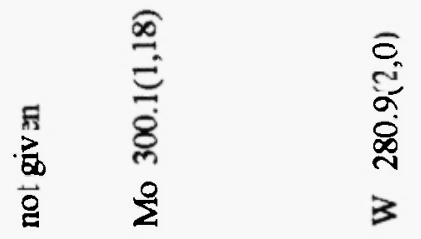

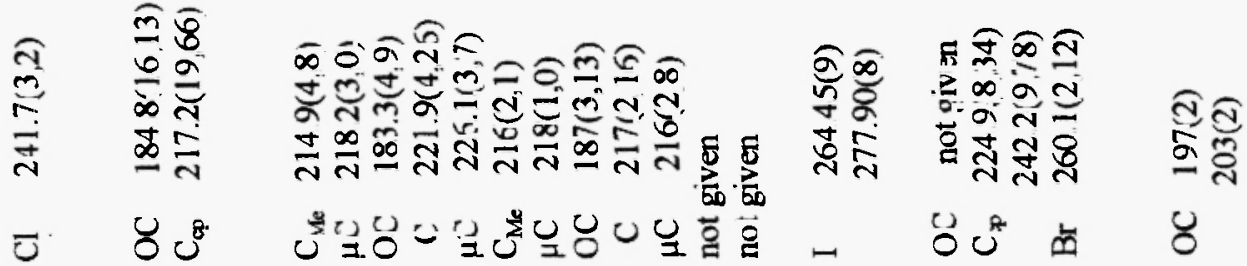

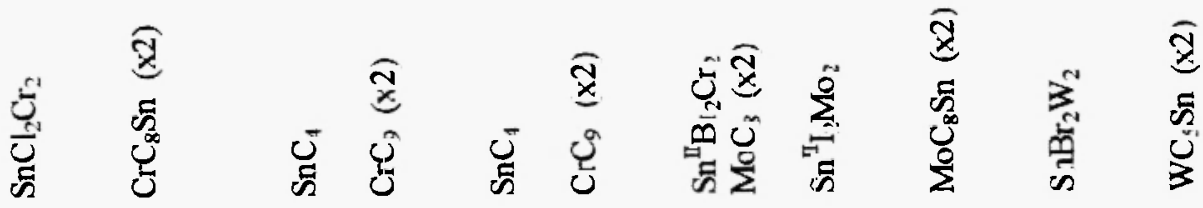

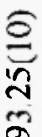
$\underset{\Xi}{\vdots}$
产

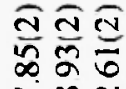
$\vDash \infty \infty$

ิิิิ)

ชิำิ

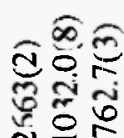

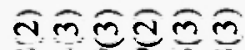

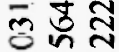

$\infty+\sin$

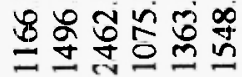

Q둥

o.

三家离

$E \stackrel{\frac{\pi}{n}}{E}$

घ $\stackrel{\frac{2}{2}}{\pi}$

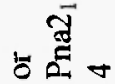

ช

$\exists \bar{a} N$

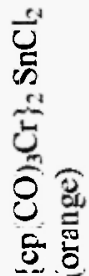
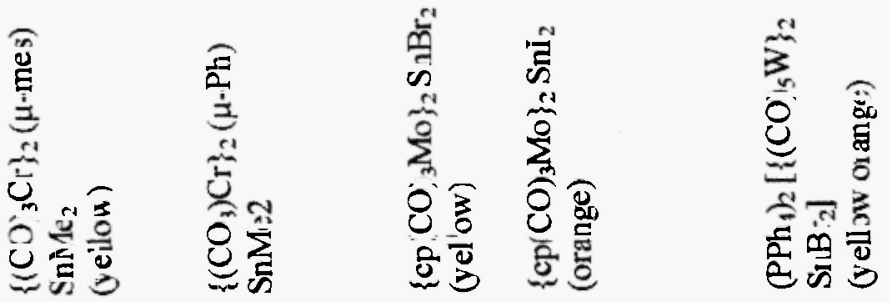
이

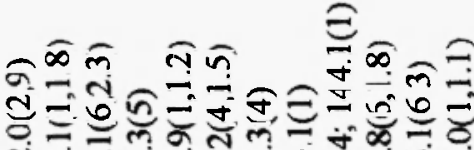

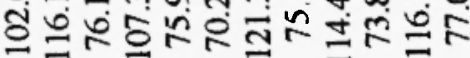
ठठ己
8

6
0
8
0
0
0

65

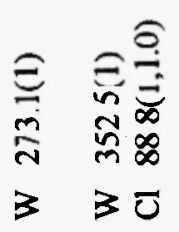

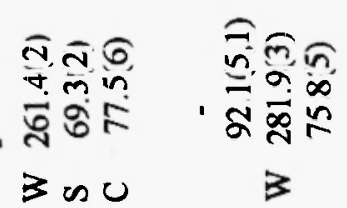

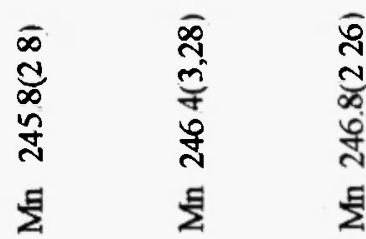

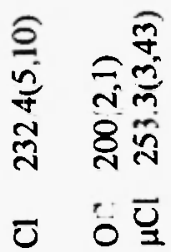

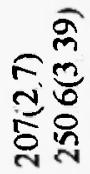

$\sum_{0}^{3}$

४⿻丁口

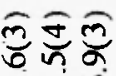

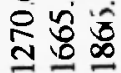

$\bigcup_{3}^{\infty}$

$5 \stackrel{0.0}{\circ} \infty$

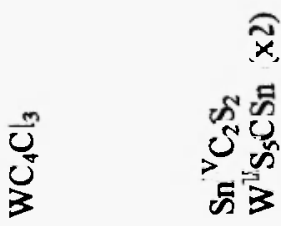

तิ $\widehat{\widehat{N}} \bar{\infty}$

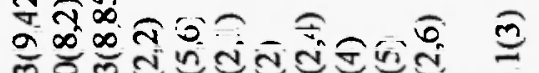

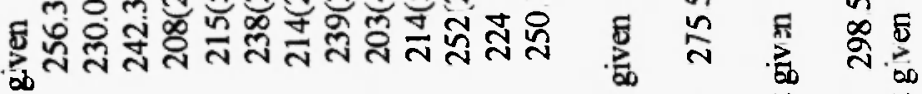

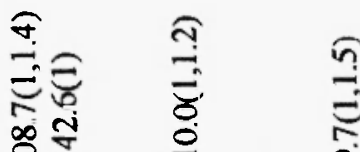

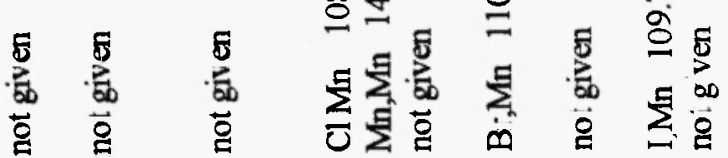

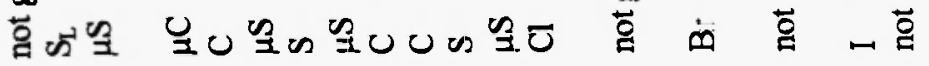

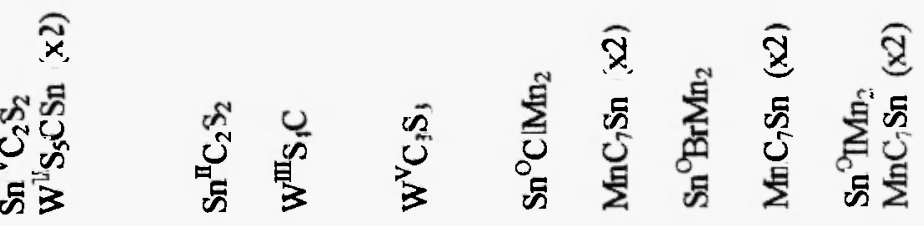

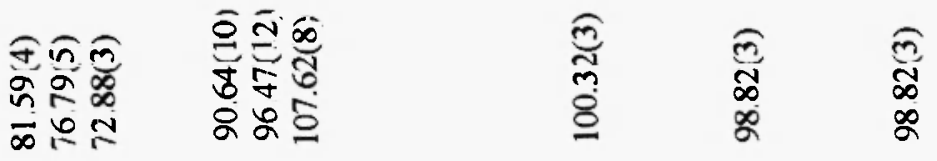

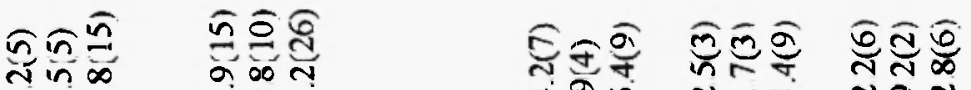

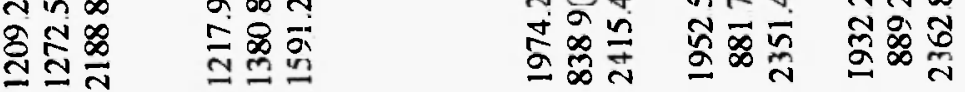

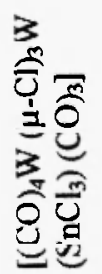

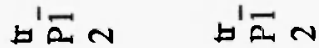

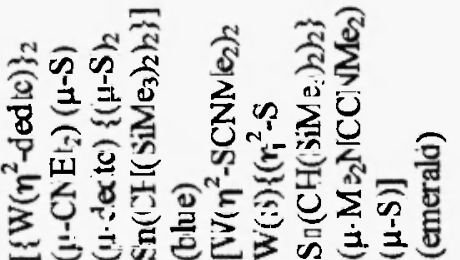

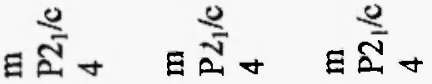

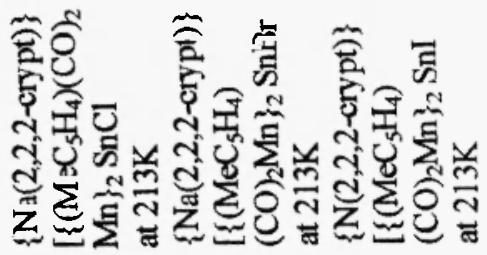




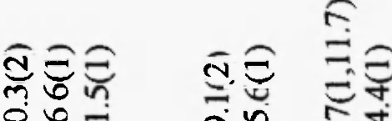

Fे

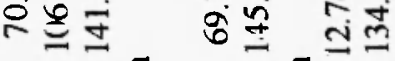

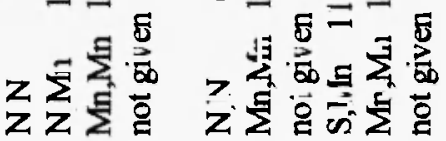

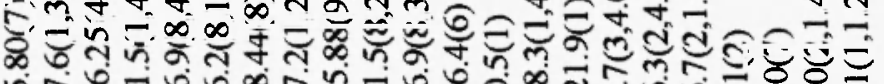

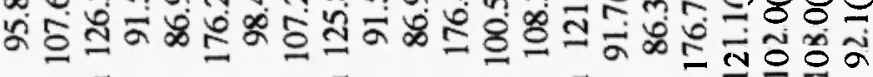

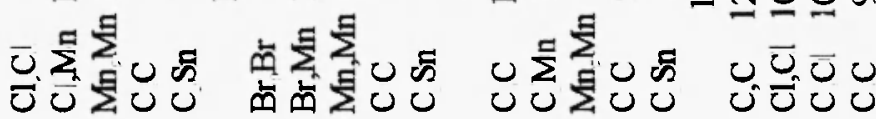

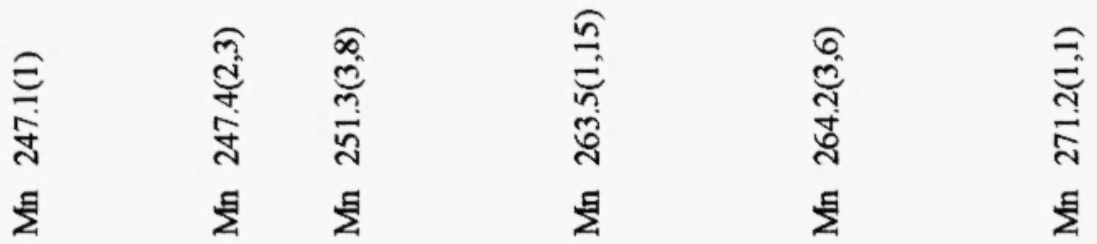

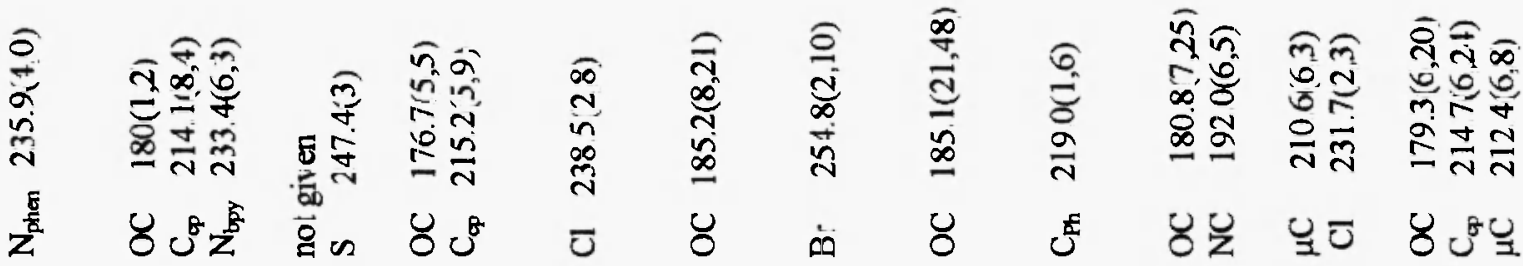

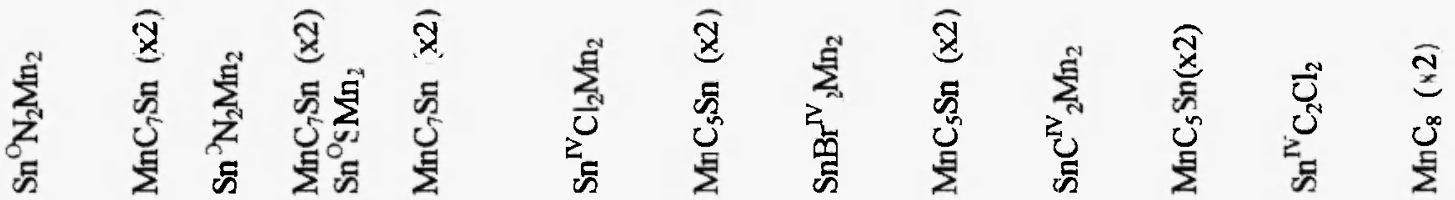
$\frac{\mathfrak{m}}{\frac{m}{a}}$

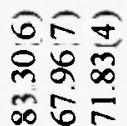
ণิ

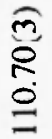
n.
$\stackrel{8}{8}$
s.

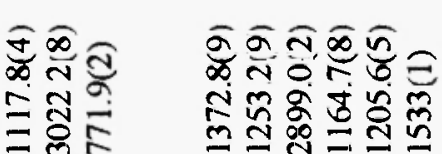

高焉

흥.

촞져용

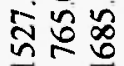

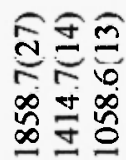

朩

客学

छ 仓ั

a $\stackrel{\text { ล }}{\text { E }}$

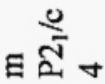

B $\stackrel{\text { ล }}{\text { ก }}$<smiles>[B]1N=[In]1</smiles>

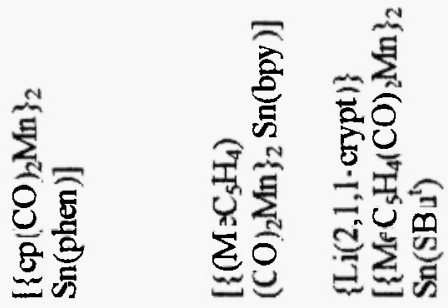

兽
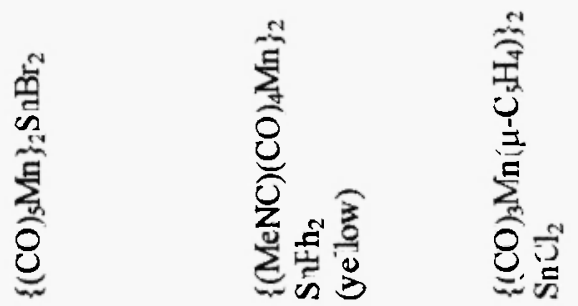
I

$$
\text { 요 } \therefore \stackrel{\infty}{\circ}
$$

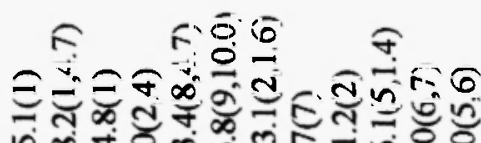

Кूँ

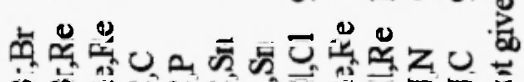

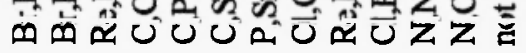

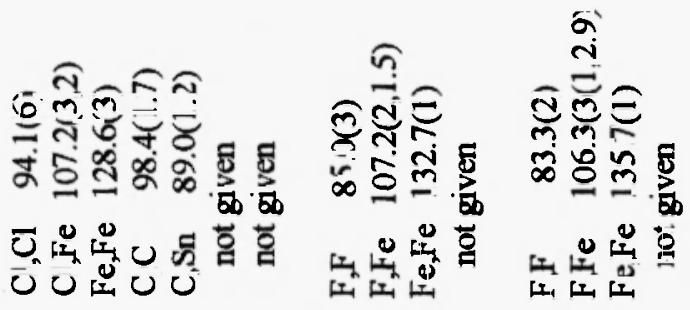

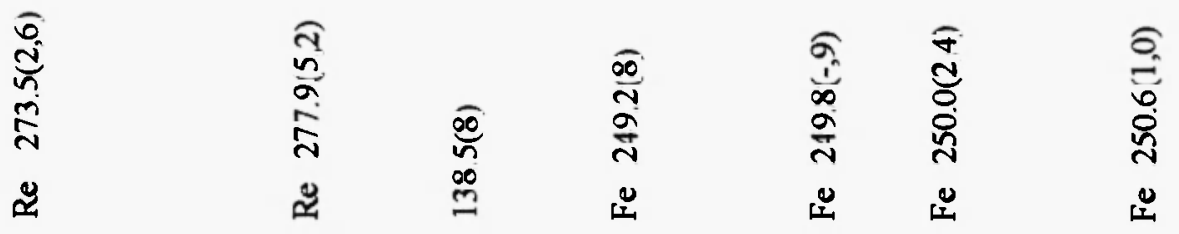

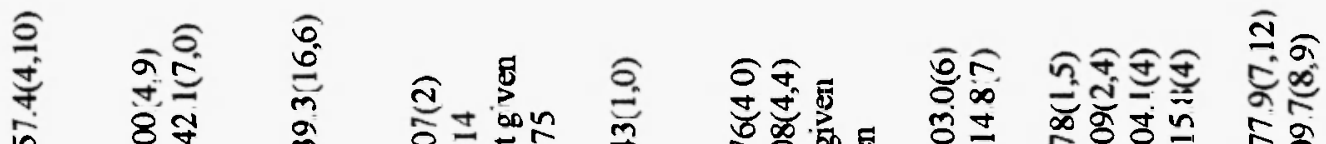

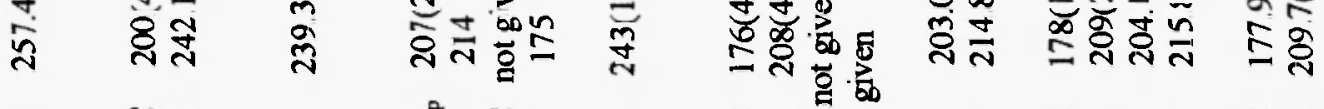

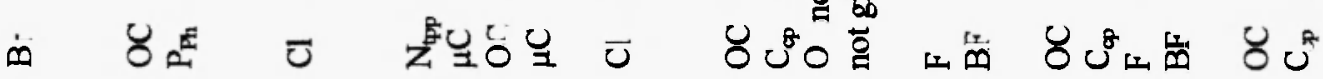

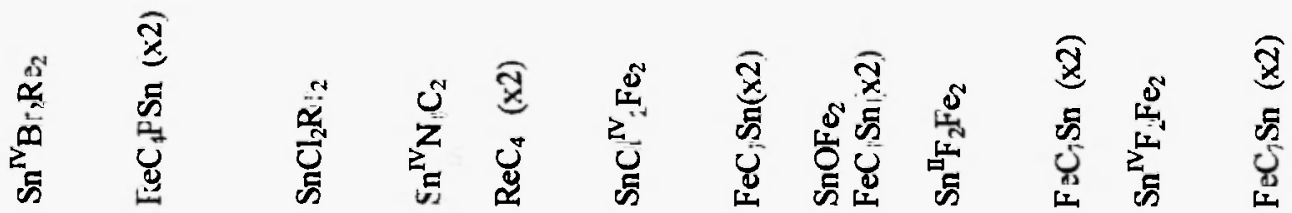

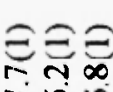

$\therefore \rightarrow$

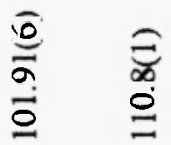

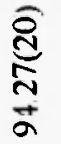

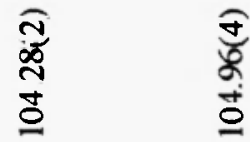

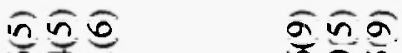

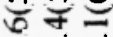

。ㅇํㅇ

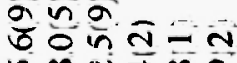

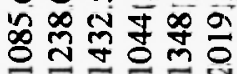

สิธิธอง

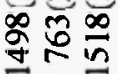

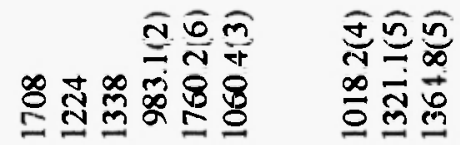

घ르

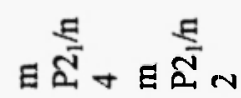

Eบั่ั

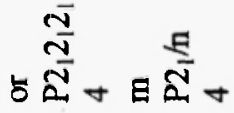

E

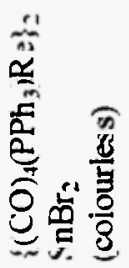

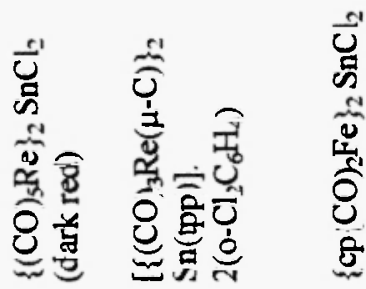
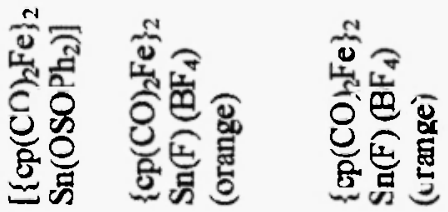
2

8

$\bar{\infty}$

$\infty$

$\infty$

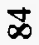

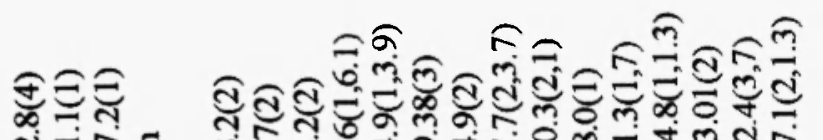

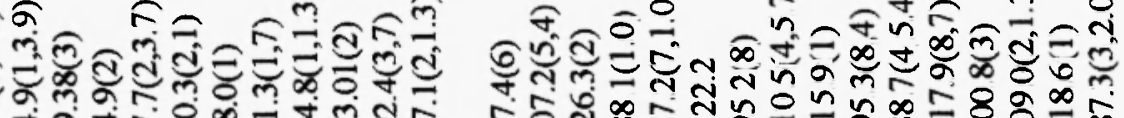

一而

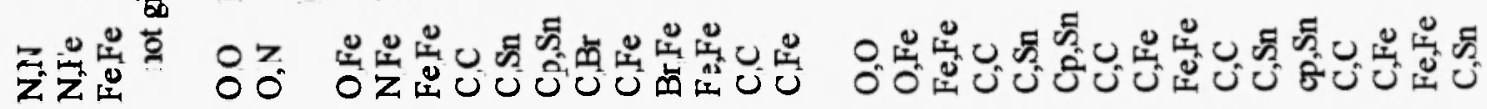

\begin{tabular}{|c|c|c|c|c|}
\hline 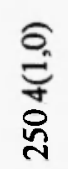 & 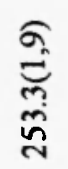 & 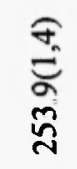 & 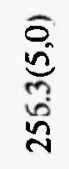 & $\begin{array}{l}\hat{m} \\
\hat{0} \\
\tilde{n} \\
\text { in }\end{array}$ \\
\hline ע & 济 & DI & $\sum_{\perp}$ & $\mathscr{L}_{\perp-1}^{\nu}$ \\
\hline
\end{tabular}

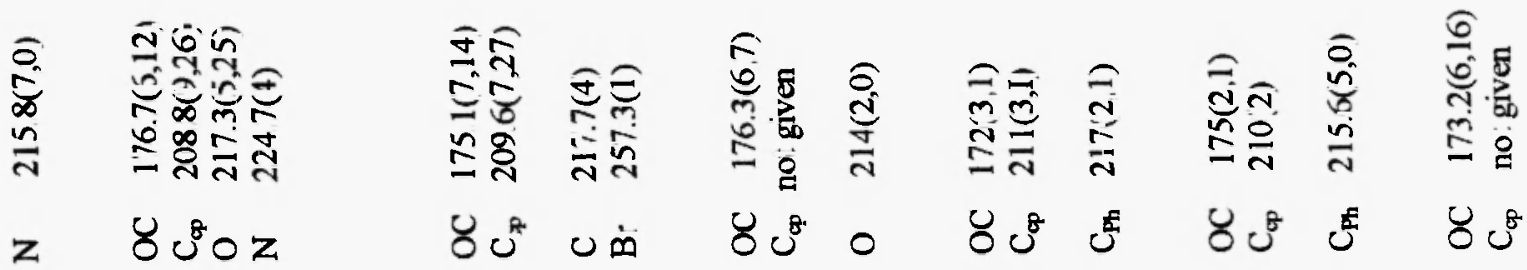

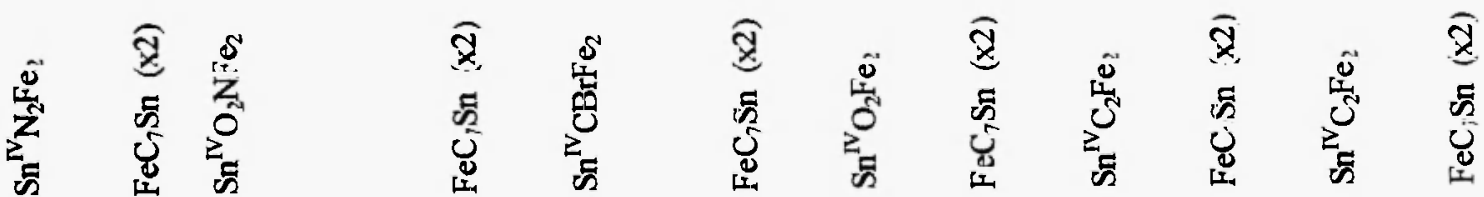

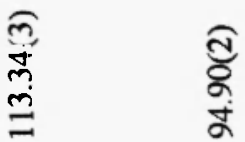

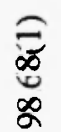

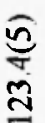
$\stackrel{\widehat{\Xi}}{\varrho}$

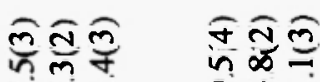

쿼ำ

达迅灾

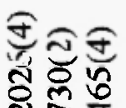

कुष

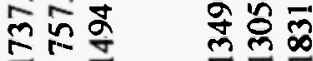

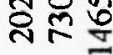

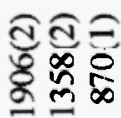

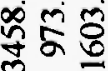

aบับั

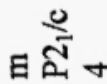<smiles>C[As]</smiles>

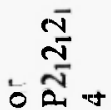<smiles>[10BH]</smiles>

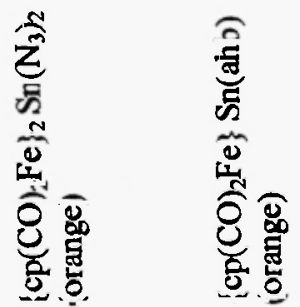

กิำ

空

о형

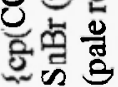
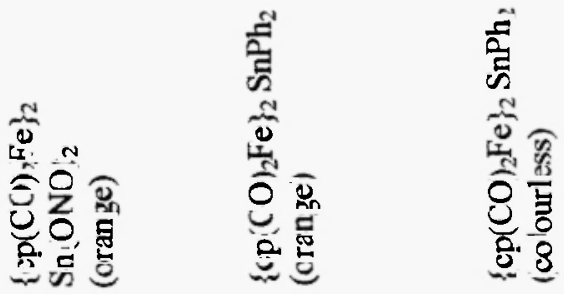
$\infty$

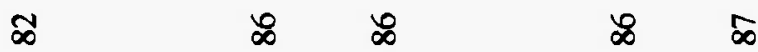

$\infty$

$\infty$

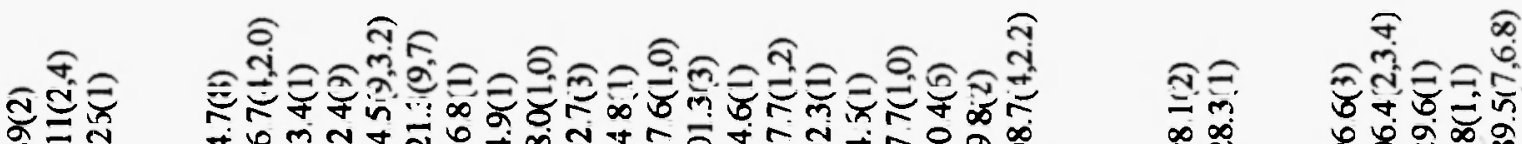

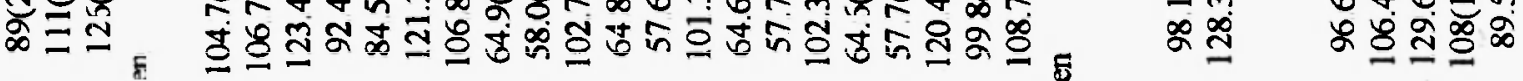

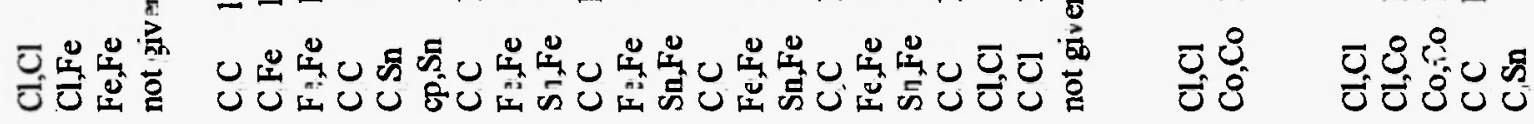

\begin{tabular}{|c|c|c|c|c|c|}
\hline 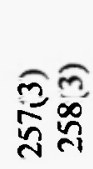 & 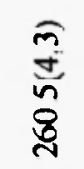 & $\begin{array}{l}0 \\
0 \\
0 \\
0 \\
0 \\
0\end{array}$ & 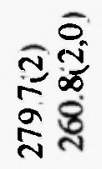 & 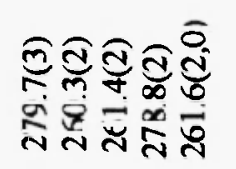 & 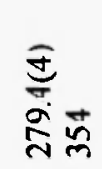 \\
\hline 平 & 里 & 里 & 品品 & 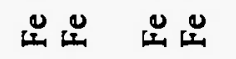 & 必怘 \\
\hline
\end{tabular}

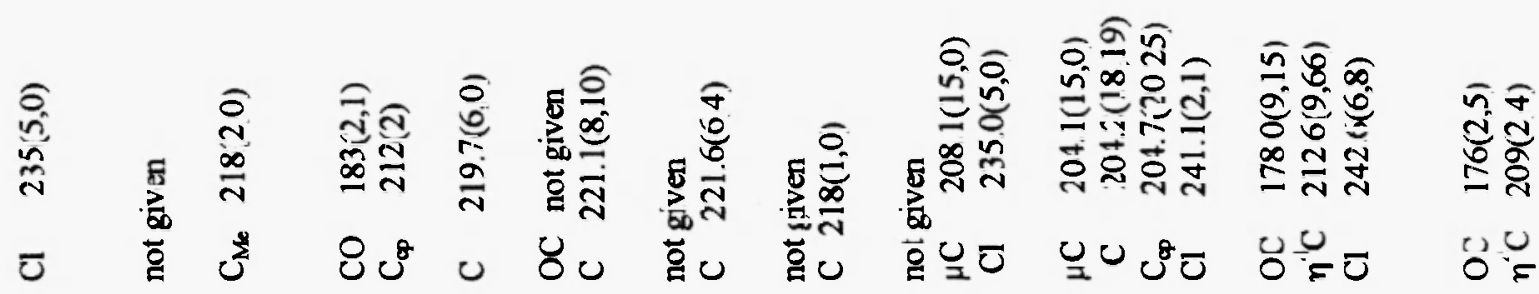

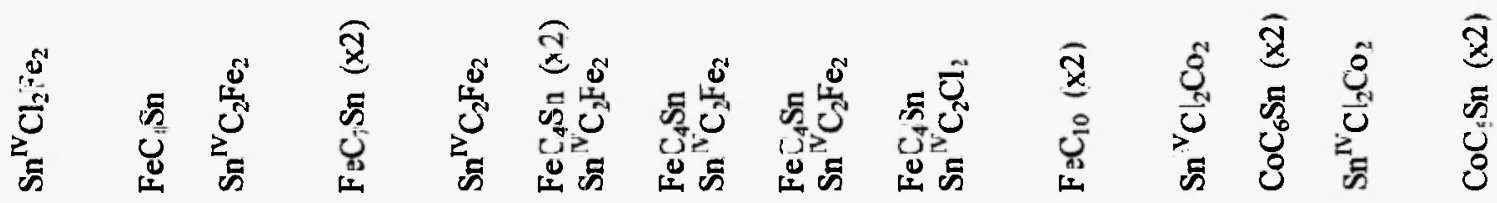

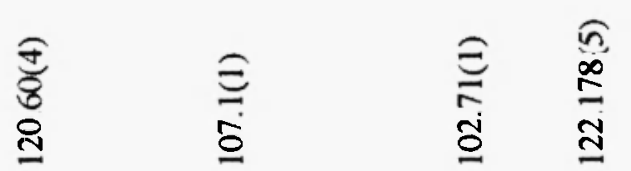

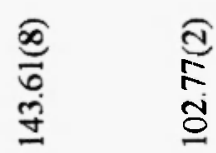

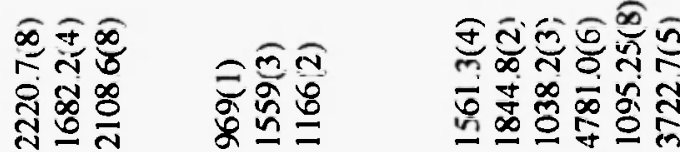

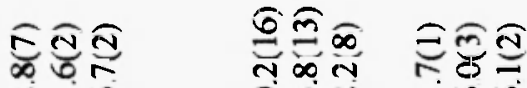

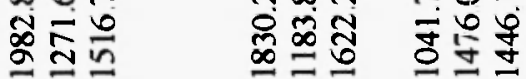

घ苂。
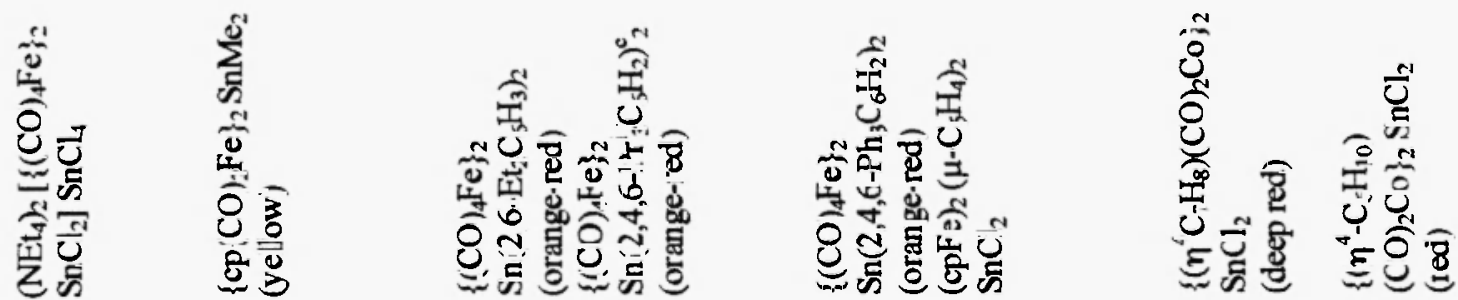
웅

$\bar{\infty} \infty \quad \delta$

a

$\nexists 2 \dddot{a}$

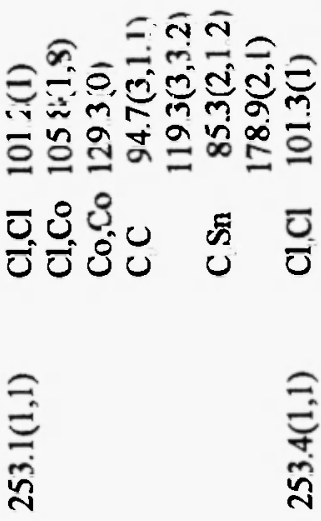

8

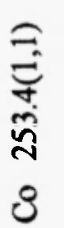

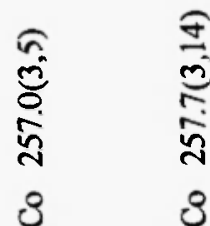

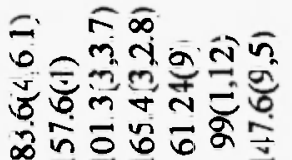

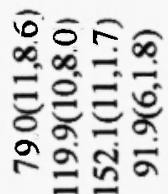

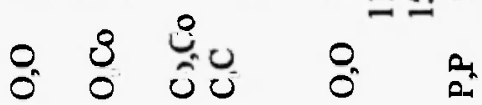

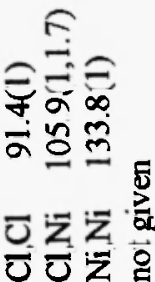

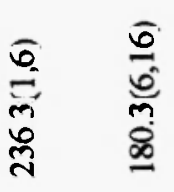

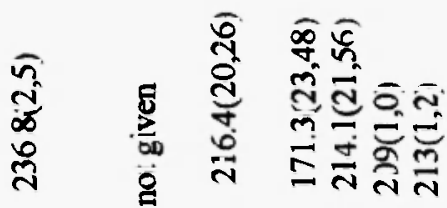

ஏ

च ठ ह ठठ

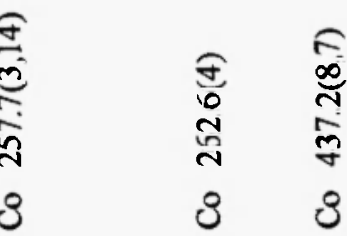

స్ำ

艺

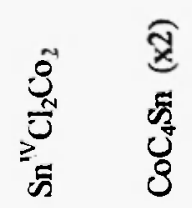

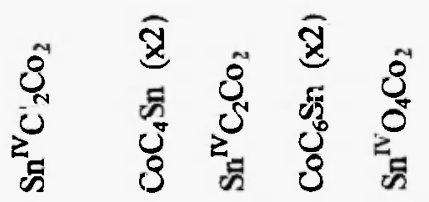

矛

$\widehat{x}$

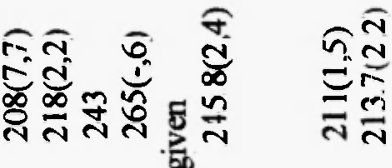

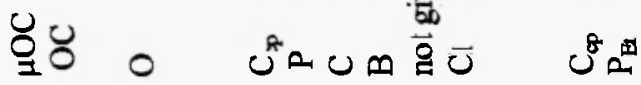

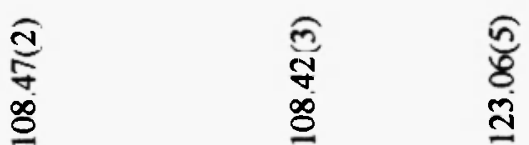

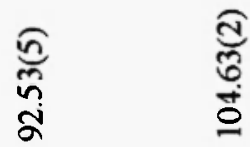

อิํำ

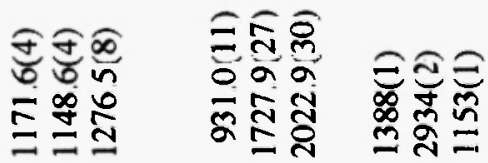

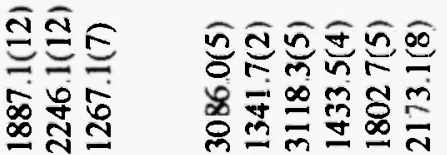

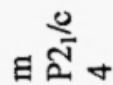

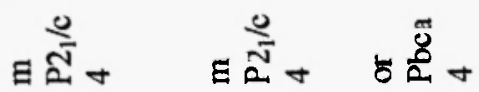

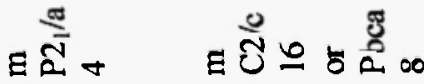
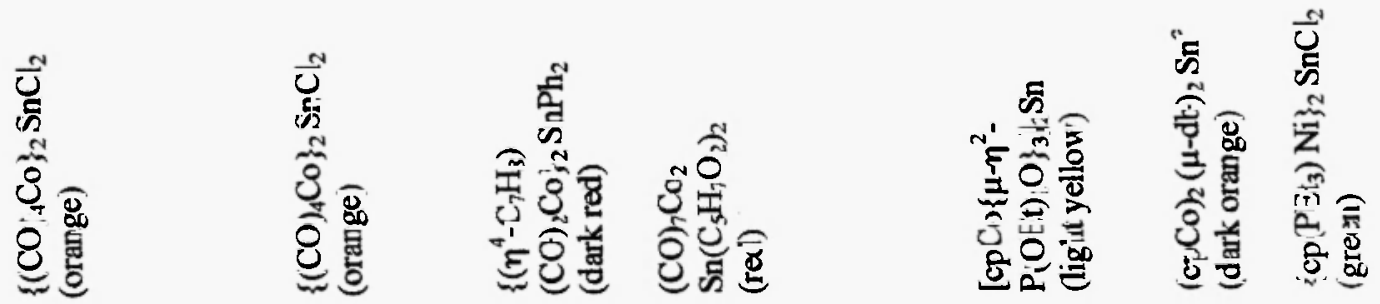
\& 5 \& 2 \&

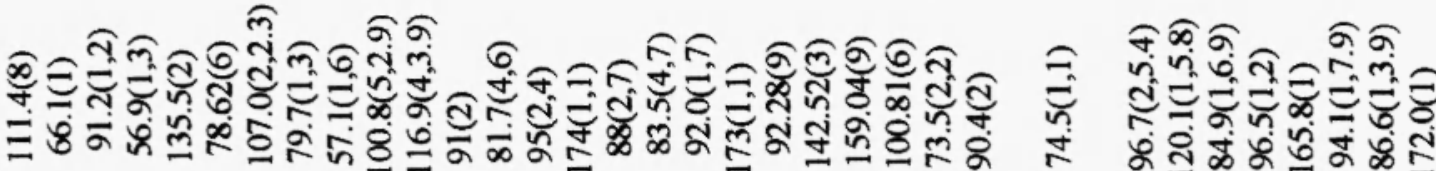

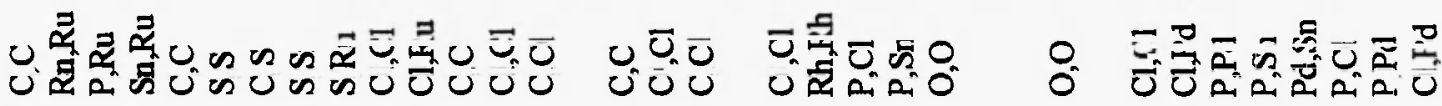

\begin{tabular}{|c|c|c|c|c|c|c|}
\hline 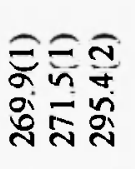 & 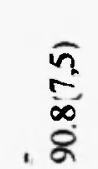 & 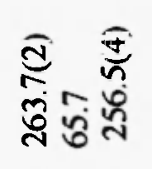 & 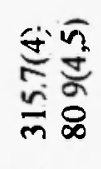 & $\frac{\sqrt{2}}{\stackrel{0}{0}}$ & $\frac{0}{n}$ & 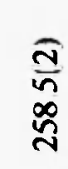 \\
\hline$\vec{z}$ & & $\vec{\approx} \quad \vec{\approx}$ & ฉี & $\tilde{x}$ & 垂 & \\
\hline
\end{tabular}

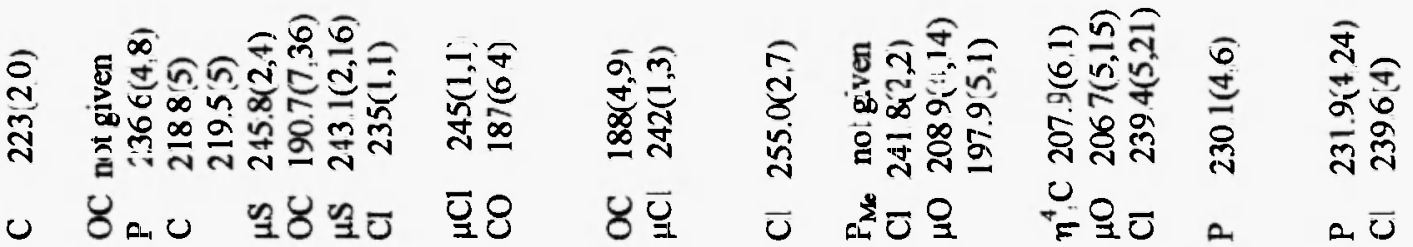

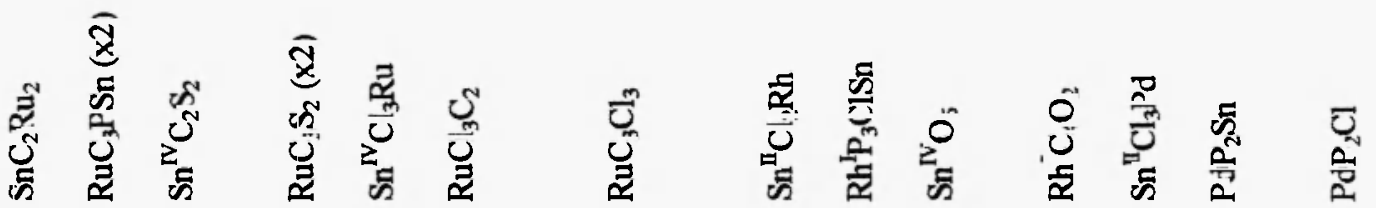

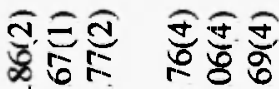
它志吉 $8 \stackrel{0}{\circ}$

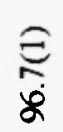

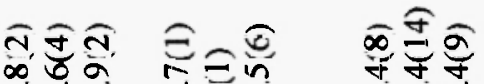

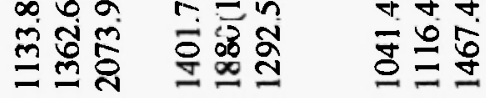

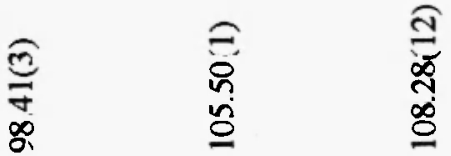

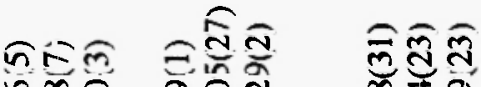

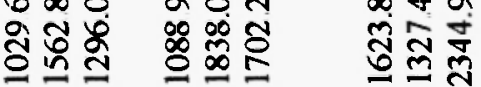

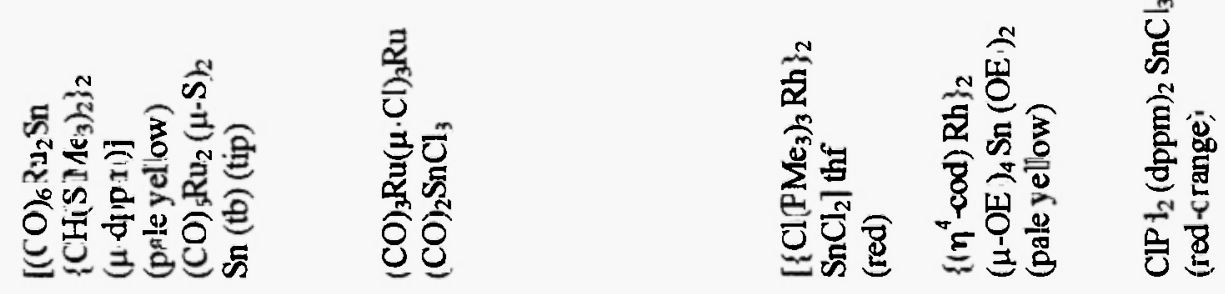


ฮั

$\dddot{9}$
肎

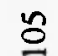

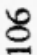

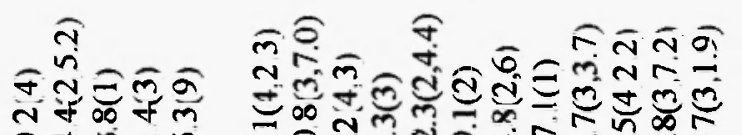

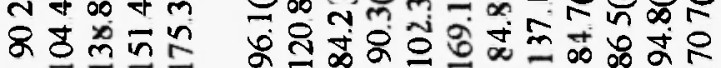

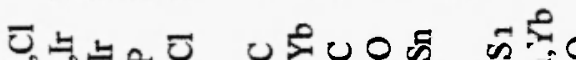

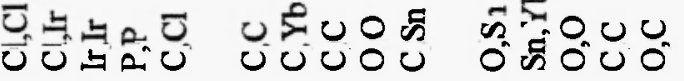

के

ڤn

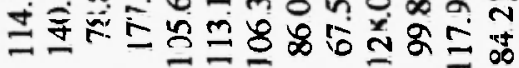

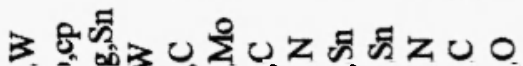

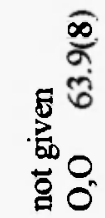

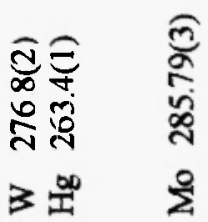

$\widehat{c}$

ㅇำ

$\sum F$

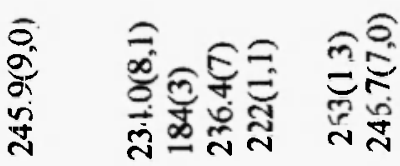

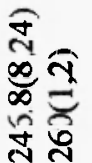

ש

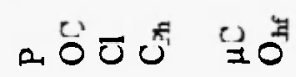

a님

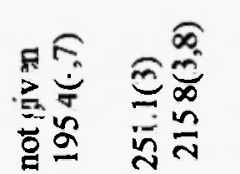

过是

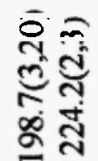

f

$8 \ddot{z}$

兽兽方

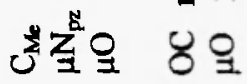

离

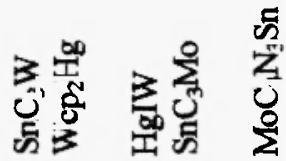

曾密

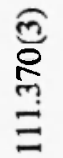

$E$
0
8
8

ํㅜㅇ

\& $\mathrm{d}=$

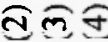

$\infty \infty$

要

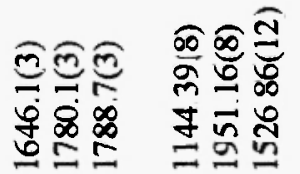

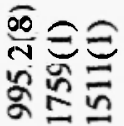

Eบั

ana

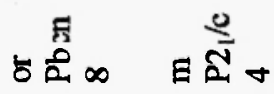<smiles>[131In][131I]</smiles>

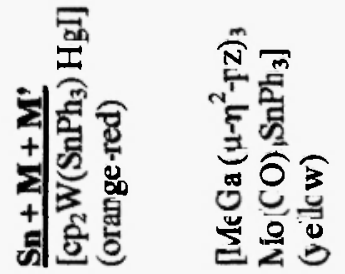

点志 


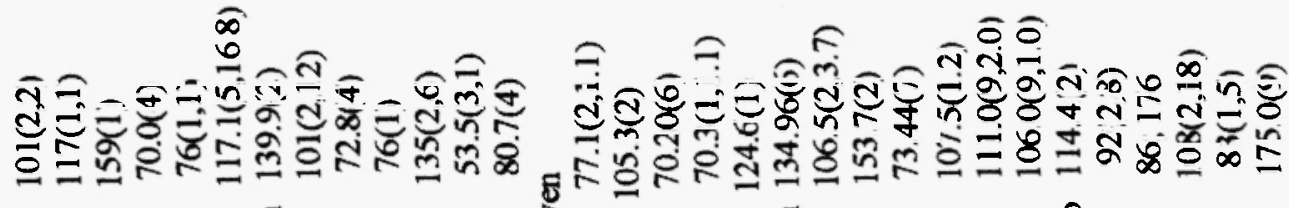

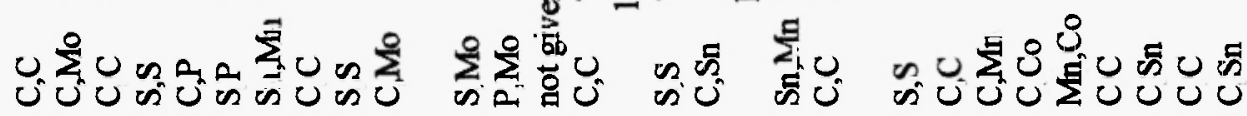

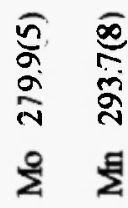

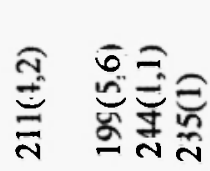

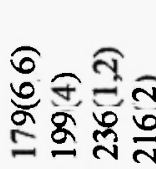

药

ㅎํ워

औ

릉

这

온론

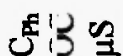

空芯

สิธิธิธ ริำ $\therefore$ ०

ลํ.

am

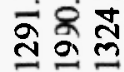

$\sin$

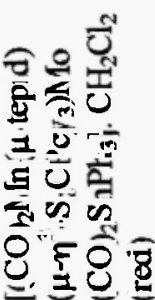

해윯

丽으

$\sum_{\substack{i \\ 0}}^{0}$

ลิกิธ

용

$\forall \bar{D}$
응응

ิㅡำ

㝵 8

$\sum_{\substack{\text { S } \\ \text { 竞 }}}^{0}$

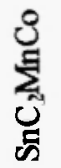

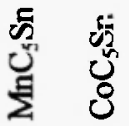

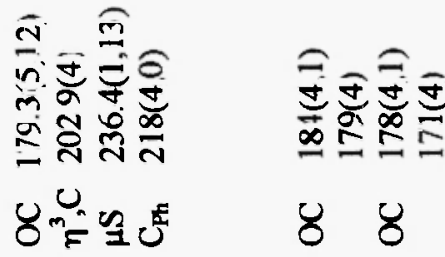

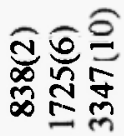

م.
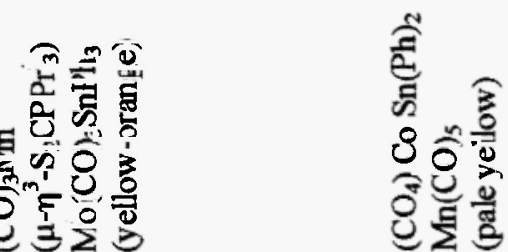
을

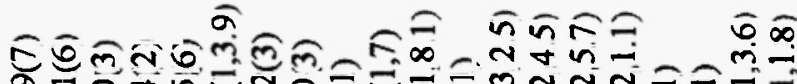

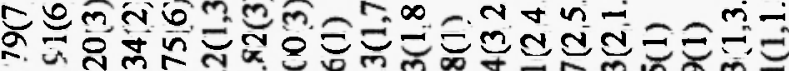

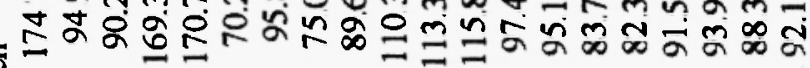

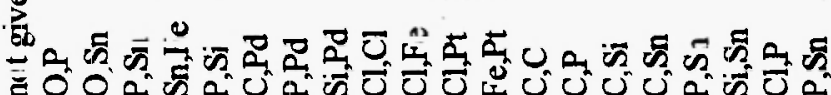

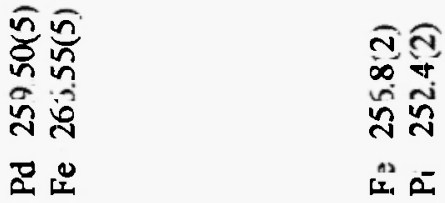

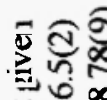

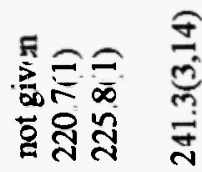

ำ ơ

음 $\frac{\infty}{N}$

$\sin$

ป $a \quad \delta a$

v

Oon in

啇

$a, \bar{v}$

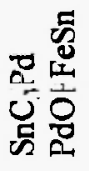

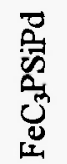

苟

号

$\frac{\overparen{\sigma}}{\sigma}$

สิธิธิ

요

$\therefore \infty \pi$

ชิํㅡㅁ

命染

-

啘势

ह $\stackrel{\frac{2}{2}}{\square}$

घอ
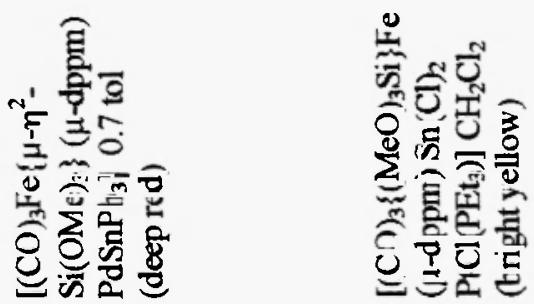
$\mathrm{P}\left(\mathrm{SnCl}_{2} \mathrm{FePt}\right)$, the platinum atom is square planar $\left(\mathrm{PtP}_{2} \mathrm{ClSn}\right)$ and the iron atom is octahedrally coordinated ( $\left.\mathrm{FeC}_{3} \mathrm{PSiSn}\right)$.

Table 1 shows that the tin atoms exist in oxidation states of zero. +2 and +4 . The coordination geometry is predominantly tetrahedral, but also includes trigonal, pyramidal and octahedral examples. The most common ligands are carbon donors (phenyl and methyl) and the chlorine atom. The mean Sn-L bond distance in the pyramidally 3-coordinated $\mathrm{Sn}(0)$ derivatives increases in the order: $248 \mathrm{pm}(\mathrm{SL})<250 \mathrm{pm}(\mathrm{CI})<275.5 \mathrm{pm}$ $(\mathrm{Br})<298.5 \mathrm{pm}(\mathrm{F})$. For the other pyramidal derivatives the order is: $220.5 \mathrm{pm}(\mathrm{CL}), 250 \mathrm{pm}(\mathrm{CI})<260 \mathrm{pm}$ (PL). For the tetrahedrally coordinated Sn(II) derivatives the Sn-L bond length increases in the order: $218.5 \mathrm{pm}$ (CL) $<244$ pm (CI). For the Sn(IV) tetrahedral derivatives the order is: 203.5 pm (F) < 218 pm (CL) < 239.5 $\mathrm{pm}(\mathrm{CI})<257.5 \mathrm{pm}(\mathrm{Br})<271 \mathrm{pm}$ (I). The mean Sn(IV)-L(bridge) bond distance increases in the order: $197 \mathrm{pm}$ $(\mu-0), 241 \mathrm{pm}(\mu-\mathrm{S})$; and $211 \mathrm{pm}\left(\mu_{3}-\mathrm{N}\right)<252 \mathrm{pm}\left(\mu_{3}-\mathrm{S}\right)$.

In heterodimeric tin compounds both non-transition and transition metals are represented. Of the former there are only 14 examples, the most common being $\mathrm{Cd}(\mathrm{II})$ [7,8,56]; $\mathrm{Li}$ [6,55]; $\mathrm{Pb}$ [7,11], $\mathrm{Sr}$ (II) [9], $\mathrm{Ba}$ (II) [10], $\mathrm{Tl}$ [106], Al(III) [57], Hg(II) [104] and Ga(III) [105]. The non-transition Sn-M distances are given in Table 1A, where the shortest is 301.3(11) pm for Sn-Li [55]. The remaining derivatives contain a transition metal.

There are several derivatives which contain two crystallographically independent molecules differing by degree of distortion in the M-L distances and bond L-M-L angles $[6,10,24,27,36,56,86,94]$. Such examples are typical of the general class of distortion isomerism [112].

Table 1A. Summary of Sn-M and M-M Distances in Heterotrimer Clusters.

\begin{tabular}{|c|c|c|c|c|}
\hline M-M' & $\begin{array}{l}\text { Cov. Radius } \\
\text { of } \mathbf{M} \text { [pm] }\end{array}$ & Shortest [ref] & $\begin{array}{l}\text { Distances } \\
\text { Longest [ref] }\end{array}$ & $\begin{array}{l}\text { Averag } \\
{[\mathrm{pm}]}\end{array}$ \\
\hline \multicolumn{5}{|c|}{$M^{\prime}=$ Non-Transition Metal } \\
\hline $\mathrm{Sn}-\mathrm{Pb}$ & 131 & $336.1(4)[7]$ & & \\
\hline $\mathrm{Sn}-\mathrm{Li}$ & 134 & $301.3(11)$ [55] & & \\
\hline Sn-Cd & 148 & $312.1(3)[7]$ & & \\
\hline Sn-Tl & 152 & $329.8(1)$ [106] & & \\
\hline $\mathrm{Sn}-\mathrm{Ca}$ & 174 & $313.2(3)$ [7] & $386.5(3)[56]$ & 353 \\
\hline $\mathrm{Sn}-\mathrm{Sr}$ & 192 & $331.9(1)$ [9] & & \\
\hline \multicolumn{5}{|c|}{$\mathbf{M}^{\prime}=$ Transition Metal } \\
\hline$\overline{\mathrm{Sn}-\mathrm{Ni}}$ & 120 & $232.6(2)[39]$ & $246.8(1)[95]$ & 240.7 \\
\hline $\mathrm{Sn}-\mathrm{Fe}$ & 120 & $249.0(1)$ [77] & $266.0(1)[30]$ & 256.5 \\
\hline $\mathrm{Sn}-\mathrm{V}$ & 125 & $275.7(3)[19]$ & $278.5(3)[19]$ & 277.1 \\
\hline $\mathrm{Sn} \cdot \mathrm{Co}$ & 126 & $244.1[36]$ & $259.1(3)[92]$ & 252.5 \\
\hline Sn-Ru & 126 & $256.5(4)[98]$ & $271.5(1)[96]$ & 266.0 \\
\hline $\mathrm{Sn}-\mathrm{Cr}$ & 127 & $254.9(1)[21]$ & $270.1(2)[60]$ & 264.0 \\
\hline $\mathrm{Sn}-\mathrm{Pt}$ & 128 & 234.3(1) [47] & $262.8(1)[52]$ & 253 \\
\hline $\mathrm{Sn}-\mathrm{Os}$ & 128 & $264.8(1)[44]$ & $271.1(1)[45]$ & 268 \\
\hline Sn-Pd & 131 & $258.5(2)$ [101] & $260.7(1)[43]$ & 259.8 \\
\hline $\mathrm{Sn}-\mathrm{Ti}$ & 132 & $281.1(2)[12]$ & $286.9(3)[13]$ & 284.5 \\
\hline $\mathrm{Sn}-\mathrm{Rh}$ & 135 & $257.1(2)[42]$ & $272.1(1)[99]$ & 264.5 \\
\hline Sn-Ir & 137 & $256.9(3)[46]$ & $275.1(1)[102]$ & 269 \\
\hline $\mathrm{Sn}-\mathrm{Nb}$ & 137 & $281.7(1)[20]$ & $286.8(1)[58]$ & 284.2 \\
\hline Sn-Mo & 145 & $270.6(1)[24]$ & $301.86(11)[57]$ & 280.8 \\
\hline $\mathrm{Sn} \cdot \mathrm{W}$ & 146 & $261.4(2)[65]$ & $280.9(2)[59]$ & 273.0 \\
\hline Sn-Mn & 146 & $243.7(2)[67]$ & $272.8(10)[109]$ & 255.3 \\
\hline $\mathrm{Sn}-\mathrm{Zr}$ & 148 & $287.15(11)[16]$ & $301.1(3)[17]$ & 294.2 \\
\hline $\mathrm{Sn}-\mathrm{Hf}$ & 149 & $295.3(1)[18]$ & & \\
\hline Sn-Re & 159 & 273.0 (1) [74] & $274.1(1)[26]$ & 273.4 \\
\hline Sn-Sn & & 276.2(1) [32] & $423.64[16]$ & 361.5 \\
\hline Fe-Fe & & 278.8(2) [86] & $279.7(2)[86]$ & 279.4 \\
\hline Co-Co & & $262.6(4)$ [92] & $437.9(8)[93]$ & 379.0 \\
\hline Ru-Ru & & 269.9(1) [97] & $315.7(1)[98]$ & 293.7 \\
\hline $\mathrm{W} \cdot \mathrm{W}$ & & $281.9(3)[66]$ & $352.5(1)[64]$ & 317.2 \\
\hline $\mathrm{W} \cdot \mathrm{Hg}$ & & $263.4(1)$ [104] & & \\
\hline Mo-Mn & & $287.7(1)[108]$ & $293.7(8)[107]$ & 290.7 \\
\hline Fe-Pd & & 266.55(6) REF?? & & \\
\hline
\end{tabular}




\section{TETRAMERIC HETEROTIN COMPOUNDS}

There are almost one hundred examples of tetrameric heterotin compounds for which the data are gathered in Table 2 . These can be divided into five subgroups based on the number of different metals present. The most common subgroup contains two tin atoms and a pair of other metal atoms. The heterometals include both transition and non-transition metals.

\subsection{Three Sn plus $M$}

There are eleven derivatives which contain three tin atoms plus one other type of metal. Only two non-transition heterometal atoms are found in this subgroup. A yellow Sn3Li derivative [113a] contains $\mathrm{Li}(\text { thf })_{4}{ }^{+}$cations and $\left[(\mathrm{Bu} \mathrm{N})(\mathrm{imn}) \mathrm{Sn}_{3} \mathrm{Li}(\mathrm{thf})\right]^{*}$ anions together with free (thf) and toluene molecules. The heterometallic anion has a cubane structure $\mathrm{Sn}_{3} \mathrm{~N}_{4} \mathrm{Li}$ in which three $\mathrm{C}_{10} \mathrm{H}_{7} \mathrm{~N}^{-2}$ imido ligands have similar environments. Each bridges the $\mathrm{Li}$ (I) centre with two $\mathrm{Sn}$ (II) centres within the cubane. The $\mathrm{Bu}^{2}$ group bridges all three tin atoms and is located at the corner opposite to the lithium atom. The coordination of the $\mathrm{Li}(\mathrm{I})$ by thf ensures that it adopts a typical pseudo-tetrahedral geometry. Each $\mathrm{Sn}$ (II) atom is 3-coordinate ( $\mathrm{SnN}_{3}$ ). In a colourless Sn3ln cluster [113] three SnMe 3 moieties and the InMe3 unit are held together by a $\mu_{4}-$ In bridge. Each metal atom has a distorted tetrahedral environment $\left(M C_{3} N\right)$.

A yellow derivative containing a Sn3 $\mathrm{Zr}_{r}$ cluster [114] has a $\mu_{4}-\mathrm{Si}$ atom serving as a bridge between three $\mathrm{SnMe}_{3}$ moieties and one $\mathrm{Zrpc} 2 \mathrm{Cl}$ unit. The tin atoms are all in distorted tetrahedral environments $\left(\mathrm{SnC}_{3} \mathrm{Si}\right)$. The zirconocene cyclopentadienyl rings are eclipsed because of the incorporation of a $\mathrm{Cl}$ and a $\mathrm{Si}$ atom into the "sandwich" cavity. There are two crystallographically independent SnzZr clusters, examples of distortion isomersim.

Another pale yellow derivative has a $\mathrm{Sn}_{3} \mathrm{Cr}$ cluster [115] in which 7-coordinate chromium is bonded to four terminal carbonyl groups and three triphenyltin units. The Sn-Cr bond distances range from 269.5(1) to 272.8(1) pm (average 271.4 pm). An orange Sn3W cluster [116] has four units, three MesSn and one W(CO)s, held together by a $\mu_{4}-\mathrm{Si}$ atom. Distorted environments are found around all the metal atoms, tetrahedral for $\mathrm{Sn}$ and octahedral for $\mathrm{W}$.

The structure of an orange $\mathrm{Sn} 3 \mathrm{Ru}$ cluster is shown in Figure 5, where it can be seen that the $\left(\mathrm{Ph}\right.$ ) SnO(Me)Sn(Phz)O(Me)Sn( $\left.\mathrm{Ph}_{2}\right)$ occupies three coordination sites in a mer orientation around $\mathrm{Ru}$. The terminal tin atoms are both tetrahedral $\left(\mathrm{SnC}_{2} \mathrm{ORu}\right)$ and the central tin atom is in a distorted trigonal bipvramidal environment $\left(\mathrm{SnC}_{2} \mathrm{O}_{2} \mathrm{Ru}\right)$ with $\mathrm{O}$ atoms at apical positions $\left(\mathrm{O}-\mathrm{Sn}-\mathrm{O}=171.1^{\circ}\right)$. The $\mathrm{Sn}(2)-\mathrm{Ru}$ distance of $267.01(5) \mathrm{pm}$ is about $6 \mathrm{pm}$ longer than the remaining two $\mathrm{Sn}-\mathrm{Ru}$ distances (260.72(5) and 261.02(5) pm). In a yellow $\mathrm{Sn}_{3} \mathrm{Pd}$ cluster [118] three $\left\{\left(\mathrm{Me} 3 \mathrm{Si}_{2}\right)_{2}\right\}_{2} \mathrm{Sn}$ moieties are held together by a $\mu_{3}-\mathrm{Pd}(0)$ atom with $\mathrm{Sn}-\mathrm{Pd}-\mathrm{Sn}$ angles of $120.0^{\circ}\left( \pm 1.2^{\circ}\right)$ and a mean Sn-Pd bond distance of $253.0(1) \mathrm{pm}$. The $\mathrm{Sn}(\mathrm{II})$ atom has a trigonal planar environment $\left(\mathrm{SnN}_{2} \mathrm{Pd}\right)$.

A yellow-orange $\mathrm{Sn}_{3} \mathrm{O}$ cluster [119] contains $\mathrm{NMe}_{4}^{+}$cations and $\left[\left(\mathrm{Cl}_{3} \mathrm{Sn}\right)_{3} \mathrm{O} \mathrm{Cl}^{-4}\right]^{-4}$ anions. In the complex anion three $\mathrm{Cl}_{3} \mathrm{Sn}$ moieties are directly bound to the $\mathrm{OsCl}$ unit with $\mathrm{Sn}-\mathrm{Os}$ distances, one of 253 and two of $258 \mathrm{pm}$. Each metal atom has a distorted tetrahedral environment $\left(\mathrm{Sn}^{\mathrm{ll}} \mathrm{Cl}_{3} \mathrm{Os}\right)$ and $\left(\mathrm{Os}^{`}{ }^{\circ} \mathrm{ClSn}_{3}\right)$.

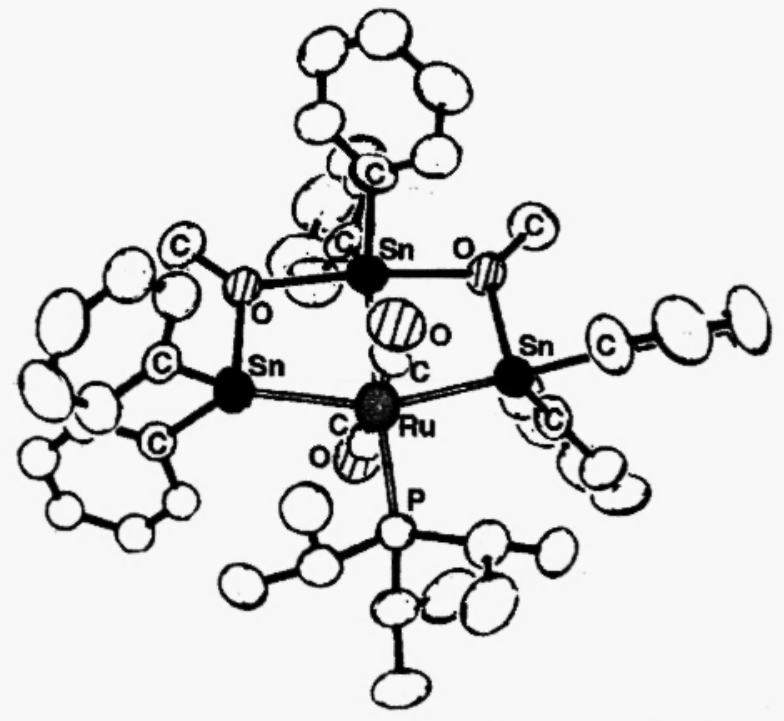

Fig.5. Structure of PhaSns $(\mu-\mathrm{OMe}) 2\left(\eta^{2}-\mathrm{H}_{2}\right)(\mathrm{CO})\left(\mathrm{PPr}^{\prime}\right) \mathrm{Ru}$ [117] 
There are three Sn3Pt clusters [120-122] in which three $\mathrm{SnCl}_{3}$ moieties are directly bound to a Pt-L unit with Sn-Pt distances ranging from 253.5(1) to 264.3(2) pm (average 258.4 pm). Each Sn(II) atom has a distorted tetrahedral environment ( $\mathrm{SnCl} 3 \mathrm{Pt}$ ) with a mean $\mathrm{Sn}-\mathrm{Cl}$ bond distance of $237 \mathrm{pm}$.

\subsection{Two Sn plus Two $M$}

\subsubsection{Non-Transition Metal $M$}

There are almost fifty clusters containing two pairs of metal atoms, two tin atoms and two of the same heterometal atoms. The latter include both transition and non-transition metal atoms. There are two $\mathrm{Sn}_{2} \mathrm{Li}_{12}$ clusters [123,124], the first has an imido Sn(II) dianion [MeNHSn $(\mu-\mathrm{Nma})]_{2}{ }^{-{ }^{-}}$associated with two [Li(thf) $]^{+}$ cations resulting in a z-shaped ladder arrangement in the core. The planar $\mathrm{Sn}_{2} \mathrm{~N}_{2}$ core of the dianion is rhombic shaped. The second example contains a $\mathrm{Sn}_{2} \mathrm{O}_{6} \mathrm{Li}_{2}$ cage which is constructed from two-semi norcubane $\mathrm{Sn}_{2} \mathrm{Li}_{2} \mathrm{O}_{3}$ units sharing a $\mathrm{Li}_{2} \mathrm{O}_{2}$ four membered ring. A colourless $\mathrm{Sn}_{2} \mathrm{Na}_{2}$ cluster [121] is isostructural with the lithium example [124] and contains two independent molecules differing by degree of distortion. The Na-Na distance of $300 \mathrm{pm}$ is about $40 \mathrm{pm}$ longer than the $\mathrm{Li}-\mathrm{Li}$ distance of $261 \mathrm{pm}$. This difference is identical with the differences between ionic radii of the four coordinate $\mathrm{M}^{+}$species, $59 \mathrm{pm}\left(\mathrm{Li}^{+}\right)$and $99 \mathrm{pm}(\mathrm{Na})$. Another colourless $\mathrm{Sn}_{2} \mathrm{Na}_{2}$ cluster [125] can be viewed as a twelve membered ring system where two $\mathrm{Sn}$ (II) and two $\mathrm{Na}$ (I) atoms are connected via four pyrazolyl functions. Two of the three pyrazol substituents at the tin atom are bridging to sodium and one is terminal. The $\mathrm{Na}(\mathrm{I})$ atoms are also coordinated to two (thf) units and one neutral pyrazol molecule.

There is a colourless $\mathrm{Sn}_{2} \mathrm{Mg}_{2}$ cluster [126] with a central $\mathrm{Mg}_{2}\left(\mathrm{OBu}^{\prime}\right)_{2}$ four membered ring spirocyclically connected to two $\mathrm{Mg}(\mathrm{OBu})_{2} \mathrm{Sn}$ rings through the common $\mathrm{Mg}$ atoms. The structure is completed by the coordination of an exocyclic tertiary-butoxy group to the terminal $\mathrm{Sn}$ (II) atoms. The $\mathrm{Mg}$ (II) atoms are therefore quasi-tetrahedrally coordinated while the $\mathrm{Sn}(\mathrm{II})$ atoms are in pyramidal three-fold Oxygen atom environments. The molecule displays a $\mathrm{Sn} . \mathrm{Mg} . \mathrm{Mg}$..Sn one dimensional arrangement with $\mathrm{Sn}-\mathrm{Mg}$ distance of 317(1) pm and $\mathrm{Mg}-\mathrm{Mg}$ distance of 293.4(1) pm.

There are six colourless $\mathrm{Sn}_{2} \mathrm{Al}_{2}$ clusters [127-130]. One of these [127] has a central four membered $\mathrm{Al}_{2} \mathrm{O}_{2}$ ring with a $\mathrm{SnPh}_{3}$ moiety bonded to each oxygen atom. The oxygen atoms are thus trigonal, linking three metal atoms, and the metal atoms have distorted tetrahedral environments $\left(\mathrm{Sn}^{\mathrm{a}} \mathrm{C}_{3} \mathrm{O}\right.$ and $\mathrm{Al}^{1} \mathrm{O}_{2} \mathrm{C}_{2}$, respectively). The Al-Al bond distance is $271.1(2) \mathrm{pm}$, but the Sn-Al distance is $347.3(1) \mathrm{pm}$ indicating no direct heterometallic bond. In the next example [128] the Sn(II) œentres are $\eta^{6}$-bonded to two molecules of benzene. the two benzene planes are inclined to each other and form angles of $101.9^{\circ}\left(\operatorname{Sn}(1)\right.$ and $99.7^{\circ}(\mathrm{Sn} 2)$, respectively. The two $\left(\eta^{\circ}-\mathrm{C}_{6} \mathrm{H}_{6}\right)_{2} \mathrm{Sn}$ units of the dimer are linked by two direct chlorine bridges and two 1,3bidentate $\mathrm{AlCl}_{4}$ groups. The coordination of the $\mathrm{Sn}(\mathrm{II})$ centres is thus pseudo-octahedral, and the entire dimer approaches non-crystallographic $C_{2 n}$ symmetry. The remaining four derivatives $[129,130]$ have a similar structure to that of the previous example [128]. The [(tol)SnClAlCl 4$]_{2}$ derivative [130] exists in two triclinic isomeric forms ( $\alpha$ - and $\beta$-) differing mostly by degree of distortion. Three $\mathrm{Sn}_{2} \mathrm{Ga}_{2}$ clusters [131-133] are colourless and contain four membered $\mathrm{Ga}_{2} \mathrm{~N}_{2}[131,132]$ or $\mathrm{Ga}_{2} \mathrm{~S}_{2}[133]$ rings. Each $\mathrm{N}$ or $\mathrm{S}$ is bonded to a $\mathrm{SnMe}_{3}, \mathrm{SnMe}_{2}$ or Sncy 3 moiety. Each metal atom ( $\mathrm{Sn}(\mathrm{IV})$ and $\mathrm{Ga}$ (III) is quasi-tetrahedrally coordinated. A colourless [cy $\mathrm{Sn}(\mu$ $\left.\mathrm{S}) \mathrm{InPh}_{2}\right]_{2}$ cluster [133] is isostructural with its $\mathrm{Ga}$ (III) analogue [133]. The Sn-In and In-In distances of 412.5(1) and $357.4(1) \mathrm{pm}$, respectively, rules out any metal-metal bonding.

In pale yellow $\mathrm{Sn}_{2} \mathrm{Ge}_{2}$ cluster [134] two heterodimers are connected by a nitrilimine group with a Sn-Ge-C-N-N-Ge-Sn arrangement. The nitrilimine linkage is almost linear (C-N-N = 174.2(11) ), but the ends of the arrangement are bent $\left(\mathrm{Sn}-\mathrm{Ge}-\mathrm{C}=94.9(4)^{\circ}\right.$ and $\left.\mathrm{N}-\mathrm{Ge}-\mathrm{Sn}=100.9(3)^{\circ}\right)$. The three methyl groups coordinated to each tin atom creates a quasi-tetrahedral environment $\left(\mathrm{SnC}_{3} \mathrm{Ge}\right)$ with an average $\mathrm{Sn}-\mathrm{Ge}$ bond distance of $265.0(2) \mathrm{pm}$. The environment about the Ge atoms is completed by $\eta^{1}$-cyclopentadienyl plus $\mathrm{CH}\left(\mathrm{SiMe}_{3}\right)_{2}$ groups giving chromophores of $\mathrm{GeC}_{3} \mathrm{Sn}$ and $\mathrm{GeC}_{2} \mathrm{NSn}$, respectively.

A black derivative [135] contains $\mathrm{K}(2,2,2-\mathrm{crypt})^{+}$cations, $\mathrm{Sn}_{2} \mathrm{Bi}^{+{ }^{+}}$anions and neutral ethylenediamine molecules. The anions have $\mathrm{Sn}$ and $\mathrm{Bi}$ in a disordered fashion over the four possible metal locations of the tetrahedral array, with bond distances ranging from 293.493) to 297.1(6) pm.

\subsubsection{Transition Metal $M$}

There are four coloured SnzTi2 clusters [136,137,15]. Two of them [136,137] contain four membered $\mathrm{Ti}_{2} \mathrm{~N}_{2}$ rings. Each nitrogen atom is also bonded to and $\mathrm{SnMe}_{3}$ unit. The nitrogen atoms are trigonal and the tin atoms quasi-tetrahedral. The skeleton structures of the other two derivatives [15] are very similar and exhibit a crystallographic centre of inversion with titanocene moieties in a staggered conformation. Two molecules of titanocene- (trimethylstannyl)-acetylide are linked by a $\pi$-bonding interaction between the titanium atom of one molecule and the acetuylide group of the other. The dimeric titanium-acetylide skeleton is essentially planar. The Ti-Ti distances, $357.3(1)$ and $363.1(3) \mathrm{pm}$ are considerably longer than the sum of the Ti 
Heterometallic Tin Compounds: Classification and Analysis of Crystallographic and Structural Data : Part II.

atom van der Waals radii $(289.6 \mathrm{pm})$, suggesting no metallic bonding.

A yellow complex $\left(\mathrm{Me}_{3} \mathrm{SnNbcp}_{2}\right)_{2}(\mu-\mathrm{O})$ [138] has a bridging oxygen atom on a crystallographic inversion centre. The linear $\mathrm{Nb}-\mathrm{O}-\mathrm{Nb}$ bridge has a $\mathrm{Nb}-\mathrm{O}$ distance of $194.34(4) \mathrm{pm}$, and the two metals are linked by a $\mathrm{Nb}-\mathrm{Sn}$ distance of $286.19(5) \mathrm{pm}$.

A blue $\mathrm{Sn}_{2} \mathrm{Cr}_{2}$ cluster [126] is isostructural with the $\mathrm{Sn}_{2} \mathrm{Mg}_{2}$ cluster in the same work and discussed previously [126]. The structure of a colourless $\mathrm{Sn}_{2} \mathrm{Mo}_{2}$ cluster [139] is composed of the $\left[\left(\mathrm{Pl}_{2} \mathrm{Sn}\right)_{2}(\mu-\mathrm{OH})\right)_{2}(\mu-$ $\left.\mathrm{MoOH})_{2}\right]^{*}$ anion, a tetrabutylammonium cation and dichloromethane solvent molecules. The two halves of the dimer are related by a crystallographic inversion centre. The complex anion contains a four membered [Sn-O(H)$\mathrm{Sn}-\mathrm{O}(\mathrm{H})$ ] ring with symmetric molybdate bridges between the tin atoms on either side of it. In addition, each $\mathrm{Sn}$ (IV) atom has bonds to two phenyl groups to complete a distorted octahedral $c r s-\mathrm{Ph}_{2} \mathrm{SnO}_{4}$ coordination.

There are three $\mathrm{Sn}_{2} \mathrm{Mn}_{2}$ clusters $[67,140,126]$. In the first [67] two $\mathrm{cp}(\mathrm{CO})_{2} \mathrm{MnSn}\left(\mathrm{S}_{2} \mathrm{C}_{2} \mathrm{H}_{4}\right)$ dimers are connected by a $\mathrm{SCH}_{2} \mathrm{CH}_{2} \mathrm{~S}$ group in the manner [MnSnSCCSSnMn], with mean $\mathrm{Mn}-\mathrm{Sn}$ bond distance of $260.53(5) \mathrm{pm}$ and $\mathrm{Mn}-\mathrm{Sn}-\mathrm{S}$ angles of $115.4(1)^{\circ}$. A second cluster [140] has a four membered $\mathrm{Sn}_{2} \mathrm{Mn}_{2}$ ring with $\mathrm{Mn}-\mathrm{Mn}$ and $\mathrm{Sn}-\mathrm{Sn}$ bond distances of $310.45(5)$ and 261.15(5) pm, respectively. The Mn-Sn-Mn and Sn-Mn-Sn bond angles are $72.94(2)$ and $106.93(2)^{\circ}$, respectively. Two phenyl groups on each tin atom complete a tetrahedral geometry about each tin atom $\left(\mathrm{SnC}_{2} \mathrm{Mn}_{2}\right)$. A pseudo-octahedral environment about the $\mathrm{Mn}$ atoms is completed by a bridging ( $\mathrm{EtO})_{2} \mathrm{POP}(\mathrm{OEt})_{2}$ ligand $\left(\mathrm{MnC}_{3} \mathrm{PSn} 2\right)$. The third pink cluster is isostructural with the aforementioned $\mathrm{Cr}$ and $\mathrm{Mg}$ analogues in the same publication [126]. The mean Sn-M and M-M distances increase in the order: $317(1)$ and $293.4(1) \mathrm{pm}(\mathrm{M}=\mathrm{Mg})<326.7(2)$ and $306.6(1) \mathrm{pm}(\mathrm{Cr})<325.6(1)$ and 306.9(1) pm (Mn). A colourless $\mathrm{Sn}_{2} \operatorname{Re}_{2}$ cluster [141] shows two $\operatorname{Re}(\mathrm{CO})_{4}$ units directly bonded with a $\mathrm{Re}-\mathrm{Re}$ bond distance of $328.9(3) \mathrm{pm}$. They are bridged by a $\mu$-oxo-distannylene $(\mathrm{Bu} 2 \mathrm{Sn}-\mathrm{O}-\mathrm{SnBu})$ group creating a five membered (Re-Re-Sn-O-Sn) ring with Re-Sn bond distances of 271.9(2) pm.

There are eight coloured $\mathrm{Sn}_{2} \mathrm{Fe}_{2}$ clusters [142-149]. Three of them [142-144] are characterised by a central lozenge shaped four membered $\mathrm{Sn}_{2} \mathrm{Fe}_{2}$ rings, with $\mathrm{Sn}$-Fe bonds ranging from 263.1(11) to 267.4(1) pm (average $265 \mathrm{pm}$ ). The mean Sn-Fe-Sn and Fe-Sn-Fe bond angles are $77^{\circ}$ and $103^{\circ}$, respectively. One of the derivatives has two crystallographically independent molecules present [142]. A red cluster [144] has two heterodimers, $\mathrm{cp}(\mathrm{CO}) \mathrm{FeSn}(\mathrm{OSOPh}) \mathrm{Ph}$, linked by a dihydroxo-bridge with asvmmetric $\mathrm{Sn}-\mathrm{O}(\mathrm{H})$ bond distances (205.4(2) and 223.1(2) pm) and Sn-O-Sn bridge angles of 102.2(2) . The Sn-Fe bond distance of 249.9(1) pm is the shortest in the series of tetrameric heterotin compounds. Each tin atom has a distorted trigonal-bipyramidal environment $\left(\mathrm{SnO}_{3} \mathrm{CFe}\right)$ with the axis defined by $\mathrm{O}-\mathrm{Sn}-\mathrm{O}\left(155.3(1)^{\circ}\right)$. The structure of another red centrosymmetric $\mathrm{Sn}_{2} \mathrm{Fe}_{2}$ cluster [146] consists of a zigzag chain of four metal atoms ( $\mathrm{Fe}-\mathrm{Sn}-\mathrm{Sn}-\mathrm{Fe}$ ) pseudotetrahedrally coordinated. The Sn-Fe and Sn-Sn bond distances are 260.5(2) and 299.1(2) pm. respectively. The Fe-Sn-Sn angles are equal at $114.9(1)^{\circ}$. A yellow example [148] consists of two ferrocene moieties linked through tin atoms in an eight membered metallocyclic central ring ( $\mathrm{Fe}-\mathrm{C}-\mathrm{Sn}-\mathrm{C}$ )2. A pair of butyl groups about each tin atom completes a tetrahedral environment about the tin atoms $\left(\mathrm{SnC}_{4}\right)$. An orange derivative [149] is similar to the yellow cluster [148] having the same central metallocyclic ring structure. The Fe-Fe separation of $547 \mathrm{pm}$ is similar to that found in the yellow derivative of $550 \mathrm{pm}$.

Two clusters contain Sn and Co $[150,126]$. The first is dark red and contains two crystallographically distinct molecules in the unit cell, each located astride a centre of inversion. but with no significant differences in molecular geometry [150]. Tin and cobalt atoms alternate around the four membered planar ring with angles at $\mathrm{Co}$ of $78^{\circ}$ and at $\mathrm{Sn}$ of $102^{\circ}$. The mean Sn-Co, Sn-Sn and Co-Co distances in the two molecules are 254.1(5), 320.695 ) and 394.4(7) pm. This indicates that the $\mathrm{Sn}-\mathrm{Co}$ is a real bond while the other two are not. Both tin atoms are in tetrahedral environments $\left(\mathrm{SnC}_{2} \mathrm{CO}_{2}\right)$. The structure of the violet $\mathrm{Sn}_{2} \mathrm{CO}_{2}$ cluster is similar to those of the $\mathrm{Mg}, \mathrm{Cr}$ and $\mathrm{Mn}$ clusters found in the same work [126]. The Sn-Co and $\mathrm{Co}-\mathrm{Co}$ distances are 320.3(1) and $298.6(1) \mathrm{pm}$, respectively.

Three $\mathrm{Sn}_{2} \mathrm{Ni}_{2}$ clusters are reported $[126,151]$. The first is similar to the $\mathrm{Co} . \mathrm{Mg}$. $\mathrm{Cr}$ and $\mathrm{Mn}$ clusters in the same publication [126] and discussed previously. The $\mathrm{Sn}-\mathrm{Ni}$ and $\mathrm{Ni}-\mathrm{Ni}$ distances are 321.1(1) and 300.9(1) $\mathrm{pm}$, respectively. The other two derivatives [151] consist of a planar $\mathrm{Sn}_{2} \mathrm{X}_{2}$ ring $(\mathrm{X}=\mathrm{S}$ or $\mathrm{Se})$ with terminal $\left.\left\{\mathrm{cp}(\mathrm{PPh})_{3}\right) \mathrm{Ni}\right\}$ and $\mathrm{Cl}$ substituents in the trans arrangement. The Sn-Ni bond distances are $242.0(1) \mathrm{pm}(\mathrm{X}=\mathrm{Se})$ and 242.4(2) $\mathrm{pm}(\mathrm{X}=\mathrm{S})$. Each tin atom has quasi-tetrahedral coordination $\left(\mathrm{SnX} \mathrm{X}_{2} \mathrm{CINi}\right)$.

A yellow $\mathrm{Sn}_{2} \mathrm{Ru}_{2}$ cluster has an almost linear central chain $\mathrm{Sn}-\mathrm{Ru}-\mathrm{Ru}-\mathrm{Sn}$ [152]. The carbonyl ligands all lean slightly towards the outer $\mathrm{SnMe}_{3}$ units with an eclipsed configuration. The Ru-Ru bond distance of 294.3(1) pm is about $25.2 \mathrm{pm}$ longer than the Ru-Os bond length (269.1(1) pm).

The molecular core of red brown $\mathrm{Sn}_{2} \mathrm{Rh}_{2}$ cluster [153] is a bent $\mathrm{Rh}-\mathrm{Sn}-\mathrm{Rh}-\mathrm{SnCl}_{3}$ chain. The central tin atom resides within the cavity of the macrocycle (twelve membered (RhPCNCP) 2 ). with coordination to two $\mathrm{N}$ atoms and two $\mathrm{Rh}$ atoms plus a terminal $\mathrm{Cl}$ atom. Each of the four metal atoms is in a unique environment. The central $\mathrm{Sn}$ (II) atom is square pyramidal $\left(\mathrm{SnN}_{2} \mathrm{Rh}_{2} \mathrm{Cl}\right)$ with $\mathrm{Cl}$ at the apex. The $\mathrm{Sn}-\mathrm{Rh}$ bond distances are $258.8(2)$ and 260.1(2) pm. The outer $\mathrm{Sn}$ (II) atom is quasi-tetrahedral ( $\mathrm{SnCl} 3 \mathrm{Rh})$ with $\mathrm{Sn}-\mathrm{Rh}$ bond distance of 258.7(3) 
pm. One $\mathrm{Rh}(\mathrm{I})$ atom is six coordinate $\left(\mathrm{RhCl}_{2} \mathrm{P}_{2} \mathrm{CSn}\right)$ and the other (RhP2Sn2C) with a Sn-Rh-C angle of $176.5(5)^{\circ}$.

\author{
$\mathrm{Rh}(\mathrm{I})$ atom is trigonal-bipyramidal
}

A yellow osmium derivative [154] contains a planar tetrametallic core of alternate $\mathrm{Sn}$ and Os atoms $\left(\mathrm{Sn}_{2} \mathrm{Os}_{2}\right.$ ). The $\mathrm{Sn}-\mathrm{Os}$ distances are 275.8(1) and 276.8(1) pm, with Sn-Os-Sn angles of 75.32(3) ${ }^{\circ}$ and $104.68(3)^{\circ}$. A pair of methyl groups about each tin atom completes the quasi-tetrahedron ( $\left.\mathrm{SnC}_{2} \mathrm{Sn}_{2}\right)$. The four $\mathrm{CO}$ groups about rhodium gives an octahedral environment $\left(\mathrm{RhC}_{4} \mathrm{Sn}_{2}\right)$.

There are two coloured Snaln clusters $[155,156]$. The first is bright green and contains a twisted tetrametallic chain of Sn-Ir-Ir-Sn with Ir-Ir-Sn bond angles of $92.9(1)^{\circ}$ and $164.2(1)^{\circ}$. Both $\operatorname{Ir}(\mathrm{II})$ atoms are bridged by a $\mu$-S atom as well as by two dppm molecules. Pseudo-octahedral coordination is completed by a carbonyl group ( $\mathrm{IrP}_{2} \mathrm{CSSnIr}$ '). Each $\mathrm{Sn}$ (II) atom is bonded to three $\mathrm{Cl}$ atoms to give a quasi-tetrahedral environment ( $\mathrm{SnCl}_{3} \mathrm{lr}$ ). The Ir-Ir bond distance of $287.8(1) \mathrm{pm}$ is about $28.5 \mathrm{pm}$ longer than the mean $\mathrm{Sn}-\mathrm{Ir}$ bond distance of 259.3(1) pm. In the second derivative [156] two Ir centres are connected by a double bridge formed by a $\eta^{\prime}$-pyrazolate group and a SBu group linked through the $\mathrm{S}$ atom. This double bridge maintains and Ir-Ir bond distance of 272.21(6) pm. Each metal centre completes its coordination sphere by three terminal ligands, a carbonyl, a trimethyl phosphite and a trichlorostannate group (IrCNPSSnlr). The Sn-Ir-Ir-Sn chain is twisted with Sn-Ir-Ir angles of $108.7(1)^{\circ}$ and $153.0(1)^{\circ}$. The Sn-Ir bond distance of $261.4(1) \mathrm{pm}$ is somewhat longer (2 pm) than that of the previous cluster [155].

Two yellow SnzPtz clusters $[157,158]$ are quite different from each other. The first consists of discrete di-chlorine bridged $\mathrm{Pt}$ dimers with each $\mathrm{Pt}$ atom bonded to $\mathrm{SnCl}_{3}$ and $\mathrm{PEt}$ moieties. These are cis to each other, but the entire molecule has a sym-trans geometry. The Pt atoms have distorted square planar environments (PtCl2PSn) with a mean Pt-Sn bond distance of 248.2(3) pm. Each Sn atom is tetrahedrally coordinated $\left(\mathrm{SnCl}_{3} \mathrm{Pt}\right)$. The second cluster [158] preserves the di-chlorine bridge but contains $\mathrm{Pt}-\mathrm{C}=\mathrm{C}-\mathrm{Pt}$ units. The unique $\mathrm{Pt}$ atom has a geometry close to square planar, with the PtCASn plane approximately normal $\left(78.3^{\circ}\right)$ to the other $\mathrm{PtCl}_{2} \mathrm{PC}$ coordination plane. Each $\mathrm{Sn}(\mathrm{II})$ atom has a distorted tetrahedral environment $\left(\mathrm{SnCl}_{3} \mathrm{C}\right)$.

\title{
3.3 Sn plus Three $M$
}

There are over thirty clusters which contain one tin atom with three identical heterometal atoms. The structural data for this group, which involves only transition metal atoms plus tin, is given in Table 2. A black SnCrs cluster [159] has a coplanar core of the four metal atoms with a mean $\mathrm{Sn}$-Cr bond distance of $266.5(2) \mathrm{pm}$ (maximum deviation $1.1 \mathrm{pm}$ ) and $\mathrm{Cr}-\mathrm{Sn}-\mathrm{Cr}$ angles all very close to $120^{\circ}$. The idealized geometry of the core is $\mathrm{D}_{3 \mathrm{~h}}$, with each chromium coordinated by five carbonyl groups. Two red SnMo3 clusters $[160,161]$ contain heterometallic cubane type $\mathrm{Mo}_{3} \mathrm{~S}_{4} \mathrm{Sn}$ cores with each metal atom six coordinate. The Sn-Mo and Mo-Mo distances range from 329.2 to $377.3 \mathrm{pm}$ and 272.3 to $300.5 \mathrm{pm}$, respectively. There are two coloured $\mathrm{SnW}_{3}$ clusters $[162 \mathrm{a}, 162 \mathrm{~b}]$. In the first the tin atom is central and coordinated to three W(CO)s units with $\mathrm{Sn}-\mathrm{W}$ bond distances of 271.3(2), 279.0(2) and 279.4(2) pm. The fourth position about each tin atom is occupied by the $O$ atom of a (thf) ligand resulting in a quasi-tetrahedral environment $\left(\mathrm{SnW}_{3} \mathrm{O}\right)$. The structure of the second. dark brown, cluster is of a cubane type with a $\mathrm{W}_{3} \mathrm{~S}_{4} \mathrm{Sn}$ core similar to that of the molybdenum case discussed above [160]. Each metal atom is octahedrally coordinated, by three $\mathrm{S}$ and three $\mathrm{N}$ atoms for tungsten and by three $\mathrm{S}$ and three $\mathrm{Cl}$ atoms for tin. The mean W-W bond distance of $276.6(2) \mathrm{pm}$ is about $3.6 \mathrm{pm}$ longer than that of the shortest Mo-Mo bond distance in the Mo analogue [160].

There are four red SnMn3 clusters [163-166] where the central atom is tin. In all four cases the Mn atoms are directly bonded to $S n$ with distances ranging from $244.5(1)$ to $275.8(2) \mathrm{pm}$ (mean $267 \mathrm{pm}$ ). In a yellow orange SnRes cluster [167] the asymmetric unit contains two similar but crystallographically independent molecules. examples of distortion isomerism. In each the three Re atoms define an approximate equilateral triangle, with the $\mathrm{Sn}$ atom bridging one edge essentially in the plane of the triangle. The Sn-Re bond distances are 266.6(3) and 287.4(4) pm in one molecule, 268.4(2) and 286.8(3) pm in the other. The MesSn bridged ReRe distances are 314.8(2) and 315.2(2) pm, respectively, and the mean value of the other Re-Re distances is shorter at $308 \mathrm{pm}$. Each rhenium atom has four terminal carbonyl groups.

There are two red $\mathrm{SnFe}_{3}$ clusters $[168,169]$ in which the four metal atoms form a core with a mean SnFe bond distance of $254 \mathrm{pm}$ (range from $252.7(1)-255.6(1) \mathrm{pm}$ ). The Fe-Sn-Fe angles are all very close to $120^{\circ}$ (maximum deviation $3.8^{\circ}$ ). One of them [169] contains two crystallographically independent clusters differing mostly by degree of distortion.

Three $\mathrm{SnC}_{3}$ clusters $[90,170,171]$ may be described in terms of a central tin atom which carries a $\mathrm{Br}$ $[170]$ or $\mathrm{Cl}[90,171]$ atom and three $\mathrm{Co}(\mathrm{CO})_{4}$ moieties. The geometry about $\mathrm{Sn}$ is quasi-tetrahedral $\left(\mathrm{SnXCo}_{3}\right)$, and the geometry about $\mathrm{Co}_{0}$ is distorted trigonal-bipyramidal with a mean Sn-Co bond distance of $260 \mathrm{pm}$ (range from 258 to $262 \mathrm{pm}$ ). The mean Co-Sn-Co bond angle is $113.7(2)^{\circ}$.

The structure of the $\mathrm{SnNi}_{3}$ cluster [95] is similar to that of the $\mathrm{SnCo}_{3}$ clusters with a mean $\mathrm{Sn}-\mathrm{Ni}$ bond distance of 252.591 ) pm (range $251.9(1)$ to $253.1(1) \mathrm{pm}$ ). The mean Ni-Sn-Ni angle of $113.7(1)^{\circ}$ is the same as 
found in the $\mathrm{SnCo}_{3}$ clusters.

There are four SnRuz clusters [172,173,97], the first is a yellow compound containing two independent molecules of essentially identical structure. The three $\mathrm{Ru}$ atoms are in a triangular cluster with only two metalmetal bonds which also have a bridging hydride ligand. A sulphide ligand bridges all three metal atoms together. Two of the Ru atoms carry three terminal carbonyl groups while the third has two carbonyls and a trichlorostannyl group which lies on the sulphur bridged side of the Ruz plane. The $\mathrm{Ru}-\mathrm{Sn}$ distance is 257.1(1) $\mathrm{pm}$ in one molecule and $258.8(1) \mathrm{pm}$ in the other. The ruthenium atoms differ in stereochemistry (Table 2) and the tin atom has a distorted tetrahedral arrangement $(\mathrm{SnCl} \mathrm{Ru})$. There are two dark red $\mathrm{SnRu}$ isomers [173] which are isostructural, one of them being shown in Figure 6. The cluster consists of a triangular array of ruthenium atoms triply bridged by the (ampy) ligand, with two short edges ( $R u-R u=287.8(1) \mathrm{pm}$ and 269.5(1) $\mathrm{pm})$. These are also bridged by hydrido and diphenylalkenyl ligands, respectively. The $\mu_{3}, \eta$-ampy ligand occupies three axial coordination sites, being linked to one $\mathrm{Ru}$ atom via the pyridinic nitrogen atom and to the other two $\mathrm{Ru}$ atoms by the exocyclic $\mathrm{N}$ atom. A SnPh 3 group is coordinated at an equatorial position to the first $\mathrm{Ru}$ atom being nearly cis to the hydride ligand. The cluster coordination shell is completed by seven terminal $\mathrm{CO}$ groups, three in axial sites trans to the (ampy) $\mathrm{N}$ atoms and four in equatorial sites. The final SnRuz cluster [97] is held by three $\mu_{3}$-bridging and one $\mu$-bridging selenide atoms. The two $\mu_{3}$-Se atoms are bound to two Ru atoms and one $\mathrm{Sn}$ atom. The $\mu$-Se atom links one Ru and one $\mathrm{Sn}$ atom. Two terminal C-donor ligands (tb and tip) complete a quasi-tetrahedral environment about the $\mathrm{Sn}$ atom $\left(\mathrm{SnC}_{2} \mathrm{Se}_{2}\right)$. The $\mathrm{Ru}$ atoms differe from each other with chomophores of $\mathrm{RuC}_{3} \mathrm{Se}_{3}, \mathrm{RuC}_{3} \mathrm{Se}_{2}$ and $\mathrm{RuCSe}_{4}$.

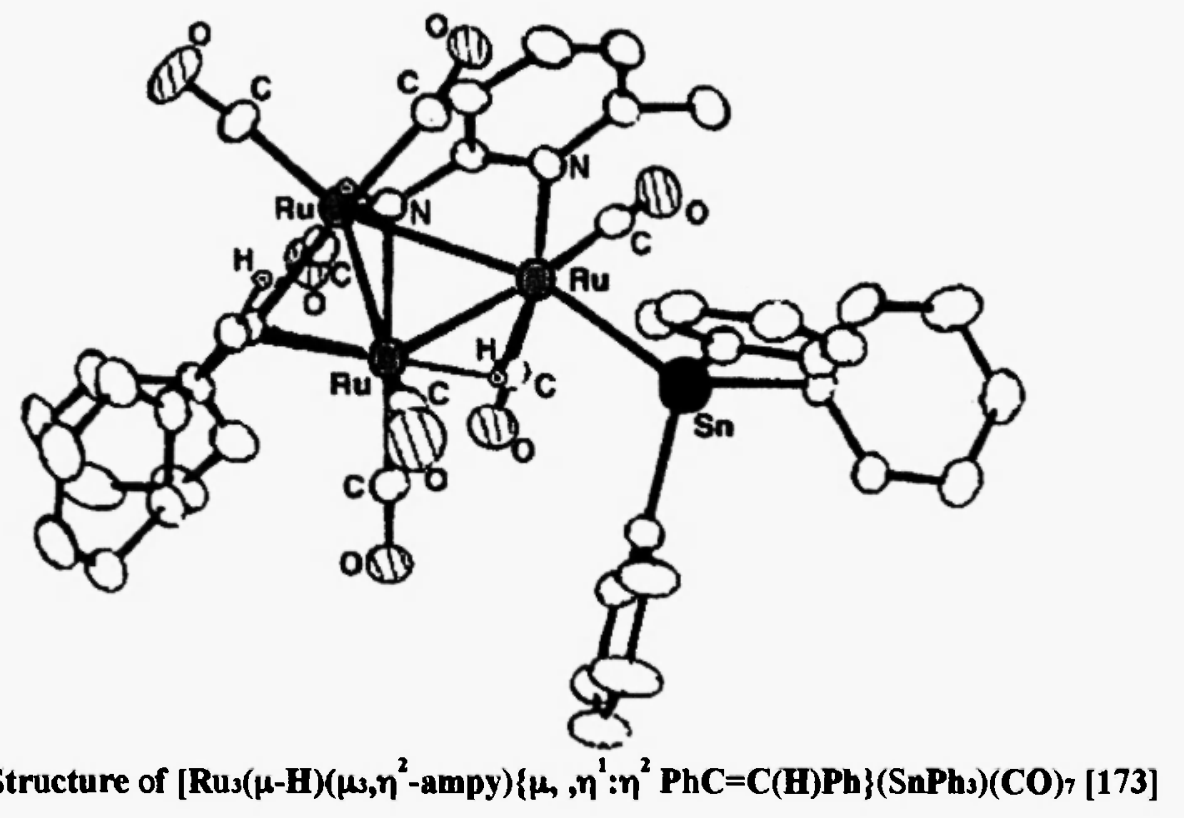

Fig.6. Structure of $\left[\mathrm{Ru}_{3}(\mu-\mathrm{H})\left(\mu_{3}, \eta^{2}-\mathrm{ampy}\right)\left\{\mu, \eta^{1}: \eta^{2} \mathrm{PhC}=\mathrm{C}(\mathrm{H}) \mathrm{Ph}\right\}\left(\mathrm{SnPh}_{3}\right)(\mathrm{CO}) 7[173]\right.$

There are eight coloured $\mathrm{SnO}$; clusters [174-178,97]. In an orange derivative [174] four metal atoms form a distorted tetrahedral array with Sn-Os bond distances of $264.1(4)$ and $264.2(4) \mathrm{pm}$, and Os-Os distances of 289.1(4) and 300.1(4) pm. Two Os atoms are bridged by a hydrido ligand. The cpy ligand is bonded via $C$ and $\mathrm{N}$ atoms to $\mathrm{Sn}$ to give a distorted tetrahedral environment ( $\left.\mathrm{SnNCOs}_{2}\right)$. The cluster coordination shell is completed by ten carbonyl groups, three of them bound to the osmium atoms which are hydrido-bridged. The remaining four are coordinated to the third Os atom. A yellow cluster [175] crystallizes in the space group P2⿺/c with two independent but structurally similar molecules per unit cell. The cluster has a nearly planar butterfly structure with the Sn and one Os atom forming the hinge. The methylene ligand asymmetrically bridges two Os atoms with Os-O distances of 205(2) and $222(2) \mathrm{pm}$. Significant asymmetry also exists in the Os-Sn bond lengths with the Sn atom located 15.9 to $17.5 \mathrm{pm}$ closer to one Os than the other two. The shorter Sn-Os distances are 264.192) pm in one molecule and 263.692) pm in the other. The Os-Os distances are 298.9(1) and $305.0(1) \mathrm{pm}$ in one case and 298.5(1) and $305.7(1) \mathrm{pm}$ in the other. The Sn atom has a distorted trigonal bipyramidal environment $\left(\mathrm{SnCl}_{2} \mathrm{Os} 3\right)$. The coordination spheres of the $\mathrm{Os}$ atoms are all different (Table 2). In another cluster shown inFigure 7 [176] a bulky alkyl group originally attached to tin ends up on the carbon of an osmium attached $\mathrm{CO}$ ligand to form a $\eta^{2}$-acyl group bridging the Os and $\mathrm{Sn}$ atoms. The (acyl) fragment spans the two metal atoms giving a basket-like configuration. Two pairs of Os atoms are each linked by hydride 
bridges. The geometry about the $\mathrm{Sn}$ atom is trigonal planar (by $\mathrm{C}$ and two Os atoms) which is then capped by the $\mathrm{O}$ atom of the (acyl) bridge.

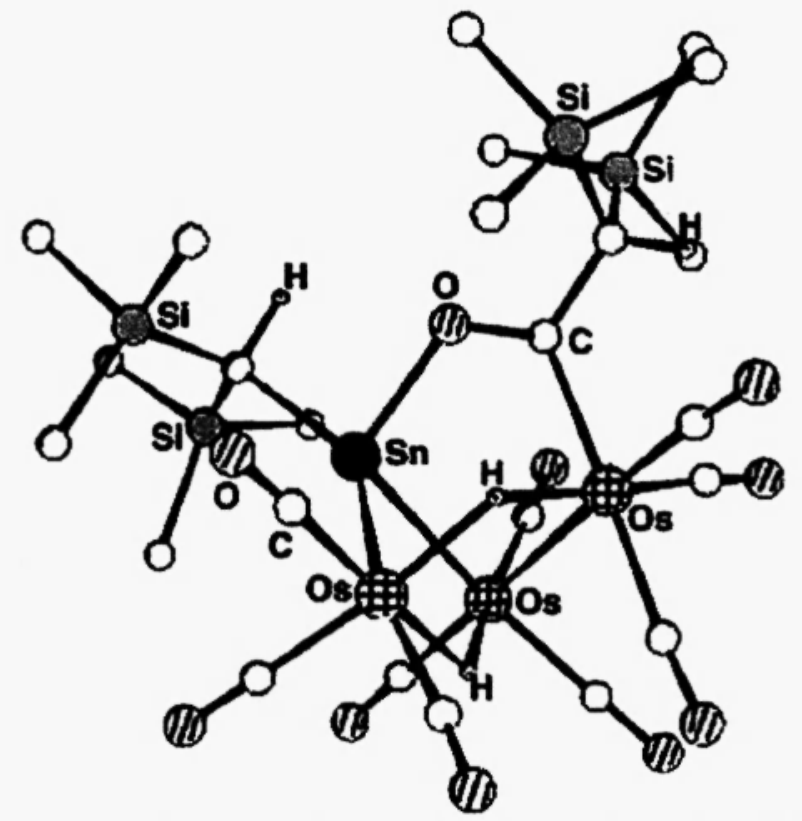

Fig. 7. Structure of OssSn( $\mu-\mathrm{H})_{2}(\mathrm{CO}),\left\{\mu-\eta^{2}-\mathrm{OCCH}\left(\mathrm{SiMe}_{3}\right)_{2}\right\}\left\{\mathrm{CH}\left(\mathrm{SiMe}_{3}\right)_{2}\right\}[176]$

The structure of another orange cluster [177] has a planar arachno-molecular metal framework with the bulky alkyl groups bonded essentially perpendicular to this plane. The yellow cluster [178] contains an open array of three Os atoms with a triply bridging sulphide ligand between them. There is also a triply bridging $\eta^{2}$ thioformaldehyde ligand in which the sulphur atom bridges two of the Os atoms and the carbon atom links to the third. The SnMes moiety is coordinated to one Os atom with an Os-Sn distance of 265.3(1) pm, with tin quasi-tetrahedral $\left(\mathrm{SnC}_{3} \mathrm{Os}\right.$ ). Two yellow derivatives [97], one with $\mathrm{S}$ and the other with $\mathrm{Se}$, are isostructural. The chalcogen atoms act as $\mu_{3}$-bridging ligands between all three Os atoms or between two Os atoms and one Sn atom. Eight terminal $\mathrm{CO}$ groups are distributed three to two Os atoms and two to the other Os atom. The cluster coordination shell is completed by two terminal C-donor ligands (tb and tip) which bond to the tin to complete a distorted tetrahedral environment $\left(\mathrm{SnC}_{2} \mathrm{XO}\right)$ s).

There are three coloured $\mathrm{SnPt}_{3}$ clusters $[179,180]$. The first contains two crystallographically independent [ $\mathrm{Sn}\left\{\mathrm{Pt}\left(\mu-\mathrm{Cl}_{2}\left(\mathrm{C}_{6} \mathrm{~F}_{5}\right)_{2}\right\}_{3}\right]$ anions. The inner platinum moiety can be described as a puckered $\mathrm{Pt}_{3} \mathrm{Cl}_{3}$ ring in which the $\mathrm{Pt}(\mathrm{II})$ atoms are interconnected by the CI atoms and acts as a tridentate donor-acceptor ligand to the $\mathrm{Sn}$ (II) atom. Each $\mathrm{Pt}$ (II) atom has a square-pyramidal coordination ( $\mathrm{PtC}_{2} \mathrm{Cl}_{2} \mathrm{Sn}$ ) with a the $\mathrm{Sn}$ atom at the apex of each. A purple complex [180] has a SnPt3 cluster cation with a $\mathrm{PF}_{0}{ }^{-}$anion. The cation contains an almost equilateral triangle of $\mathrm{Pt}$ atoms and a $\mu_{3}-\mathrm{SnMe}\left(\mathrm{PO}_{2} \mathrm{~F}_{2}\right)$ moiety. Two methyl groups, the $\mathrm{PO}_{2} \mathrm{~F}_{2}$ moiety and three $\mathrm{Pt}$ atoms complete a roughly octahedral coordination about the $\mathrm{Sn}$ atom ( $\mathrm{SnC}_{2} \mathrm{FPt}_{3}$ ). The $\mathrm{Sn}-\mathrm{Pt}$ bond distances are 270.2(2), 273.9(2) and 276.6(2) pm. The Pt-Pt bond distances are shorter at 260.9(1), 261.5(1) and 263.5(1) pm.

\subsection{Sn plus Two $M$ and $M^{\prime}$}

There are five clusters of this type [106,168,181-183] with two identical heterometals plus one different, and their crystallographic and structural data are given in Table 2. Both transition and non-transition heterometal atoms are observed.

A dark purple derivative [181] contains a bent four metal atom unit (tpp)SnMn(CO) $4 \mathrm{HgMn}(\mathrm{CO})$ s extended over the porphyrin ring with a $94.5(1)^{\circ}$ bend at $\mathrm{Sn}-\mathrm{Mn}-\mathrm{Hg}$. The $\mathrm{Mn}-\mathrm{Hg}-\mathrm{Mn}$ link bends up away from the ring slightly $\left(164.4(1)^{\circ}\right)$. The $\mathrm{Sn}$ atom is five coordinate in a square-pyramidal environment. The two $\mathrm{Mn}$ atoms are octahedrally coordinated by Co groups and metal atoms. The $\mathrm{Sn}-\mathrm{Mn}, \mathrm{Mn}-\mathrm{Hg}$ and $\mathrm{Hg}-\mathrm{Mn}$ bond distances are 255.4(3), 258.2(3) and 265.9(3) pm, respectively.

Four metal atoms are arranged nearly linearly, (CO)s $\left.\mathrm{Mo}-\mathrm{Sn}(\mathrm{m}-\mathrm{OBu})_{3}\right)_{3} \mathrm{ln}-\mathrm{Mo}(\mathrm{CO})$ s in another complex [106]. The intermetallic angles are $174.2^{\circ}$ (Mo-Sn-In) and $178.8^{\circ}$ (Mo-In-Sn). The metals are linked by single 


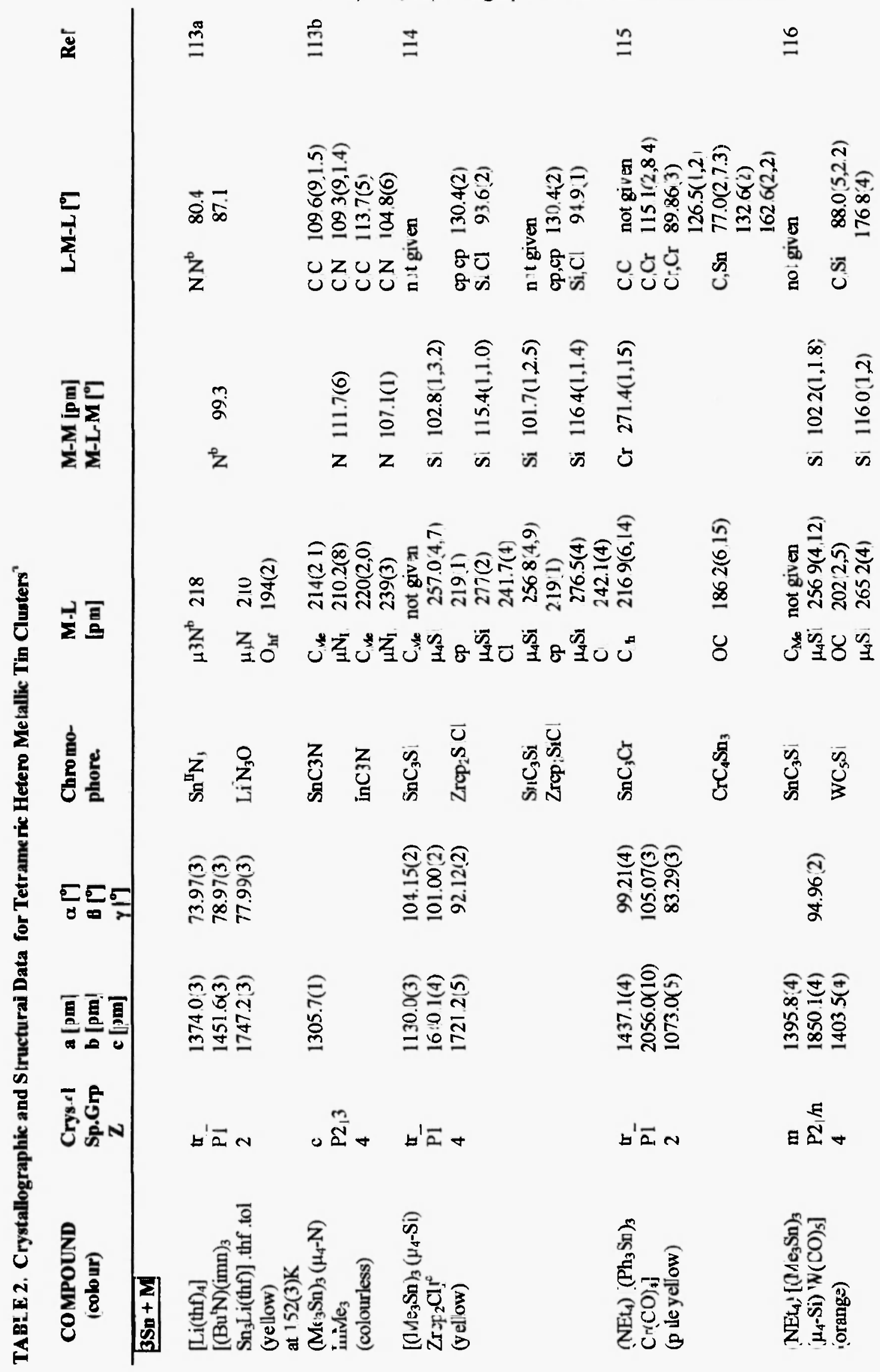


$\cong \quad \infty \quad \cong \quad \cong \quad \cong \quad \cong$

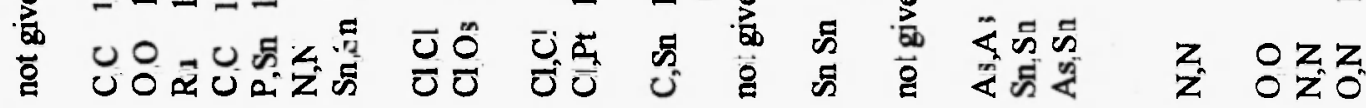

ำ

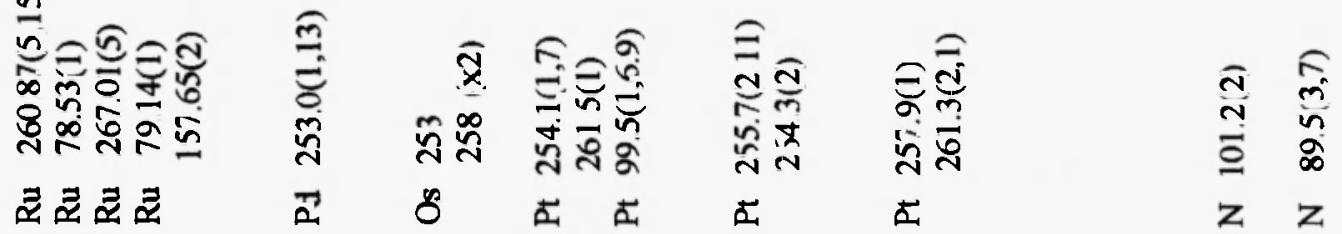

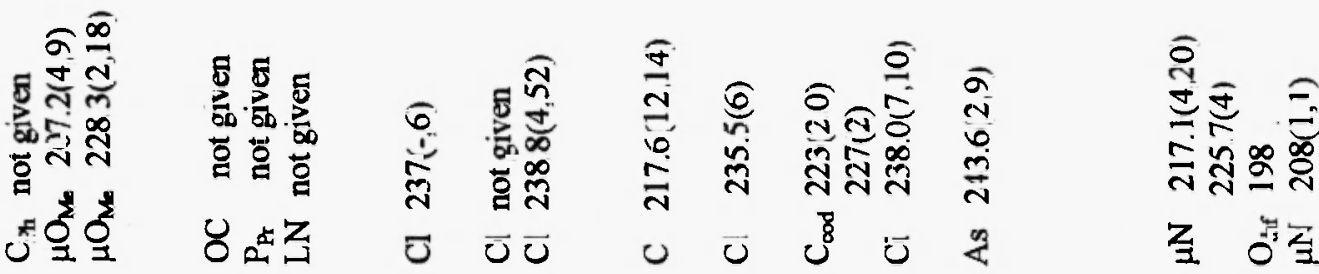

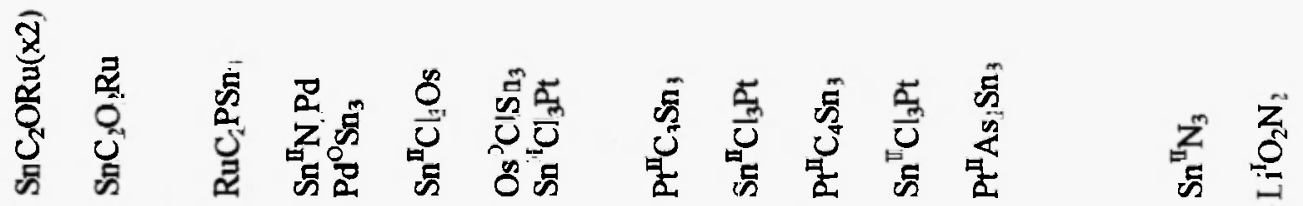

\begin{tabular}{|c|c|c|c|}
\hline 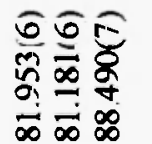 & 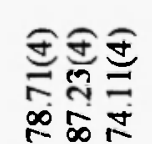 & 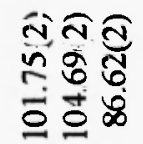 & 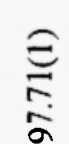 \\
\hline
\end{tabular}

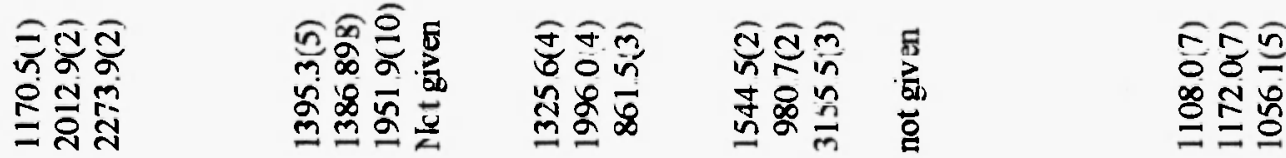

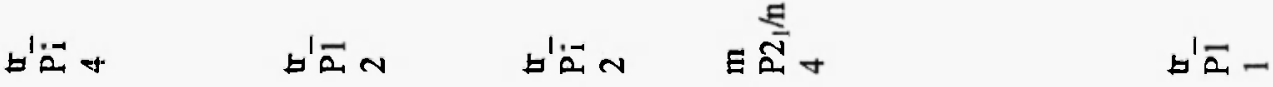

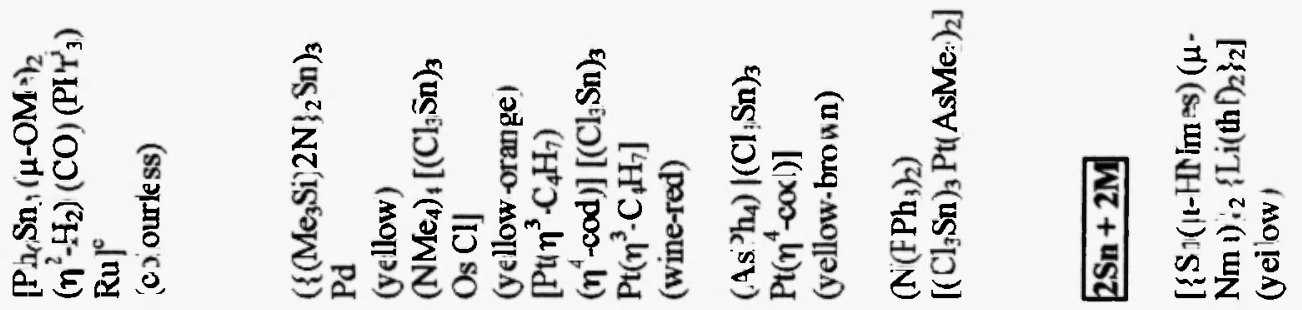




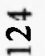

$\cong$

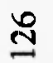

ฐ

$\stackrel{\infty}{\stackrel{\infty}{4}}$

สู้

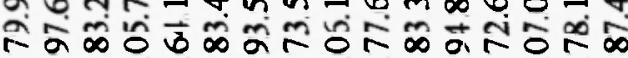

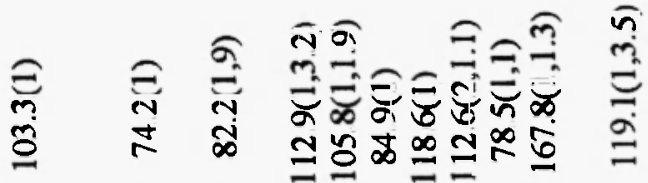

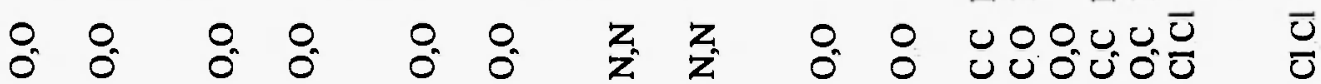

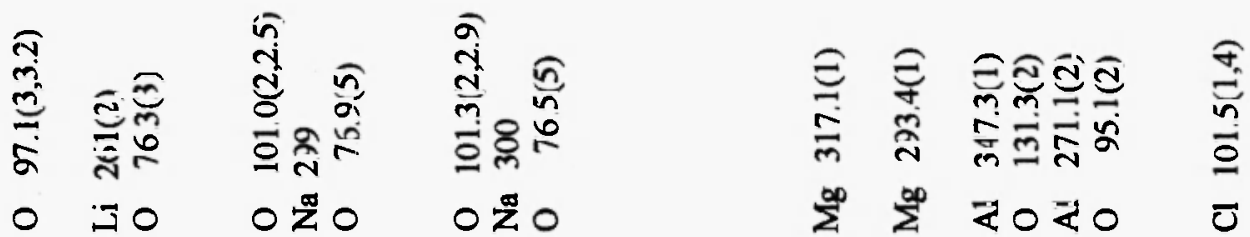

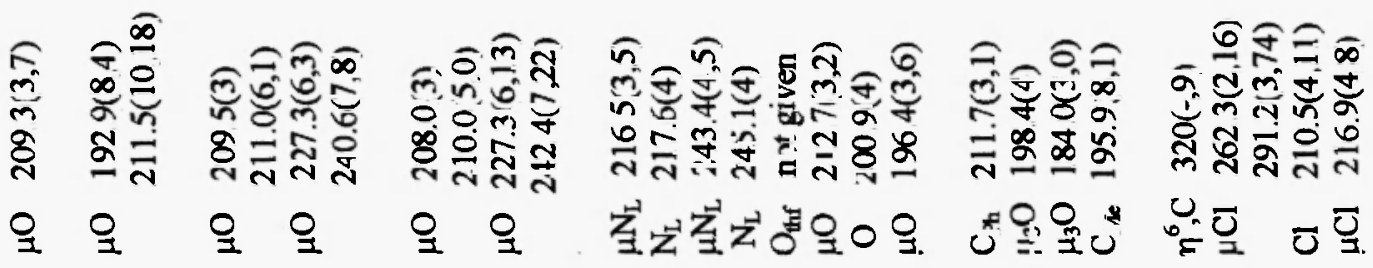

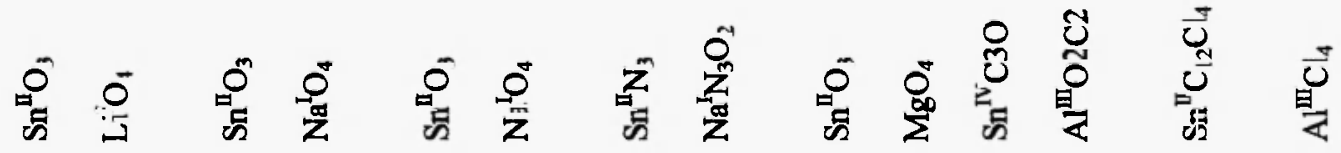

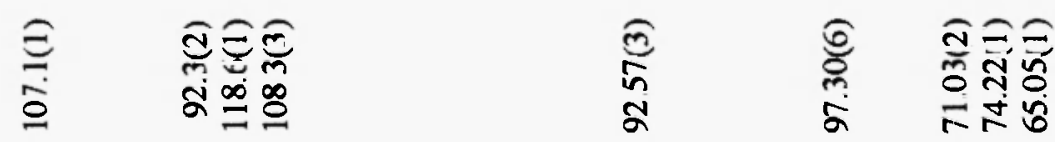

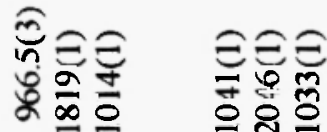

a

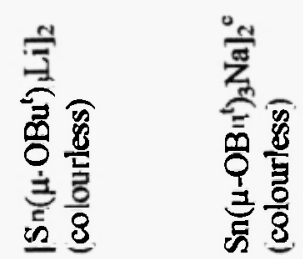

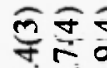

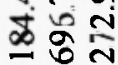

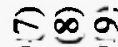
oิ-

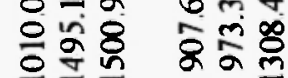

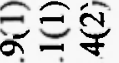
क范出 $2 \underline{x}$

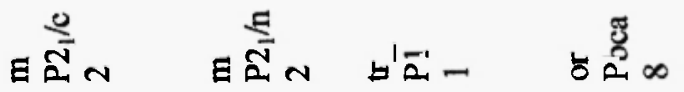
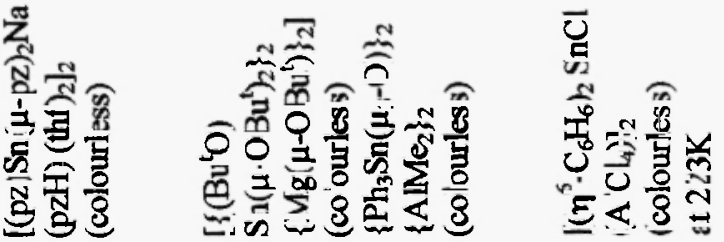


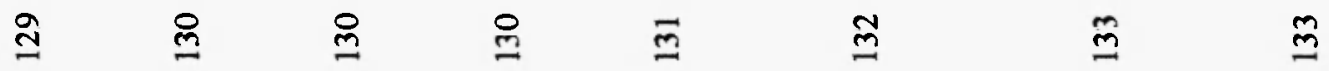

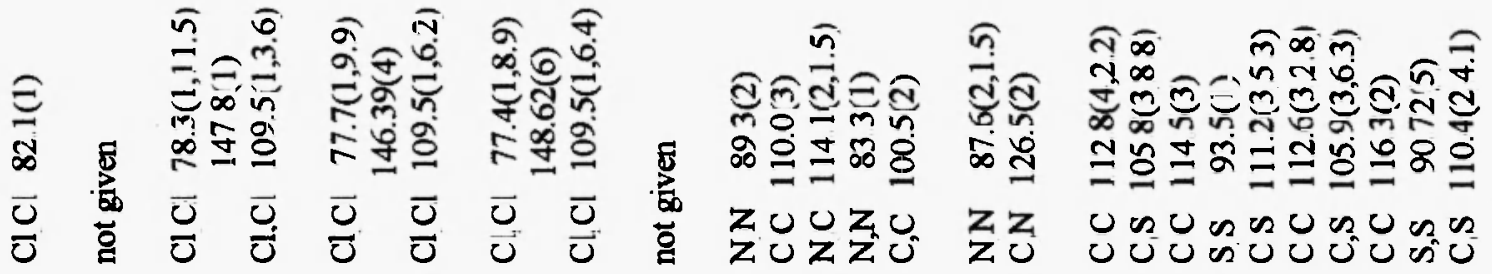

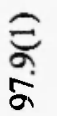

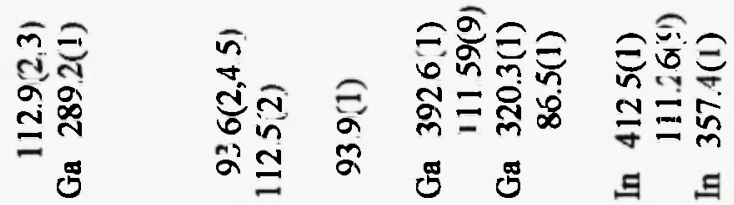

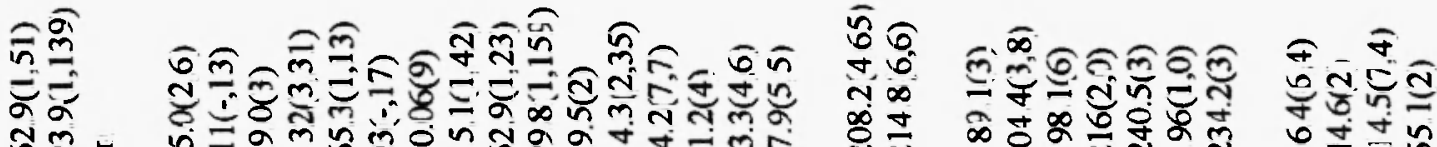

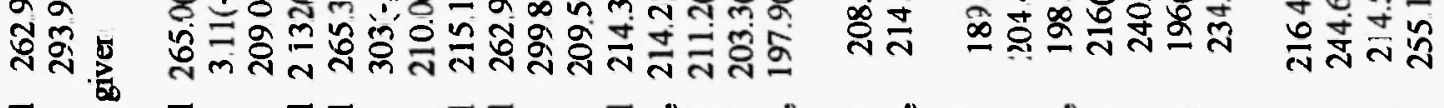
ஏ

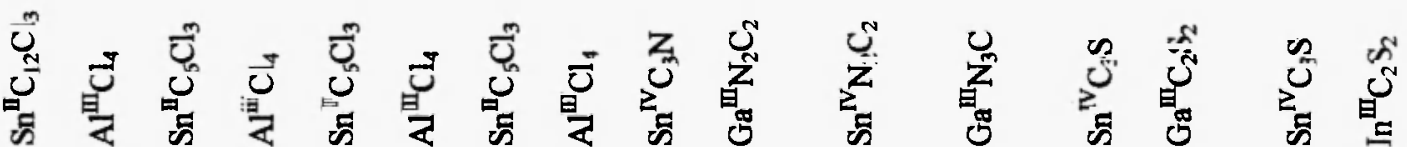

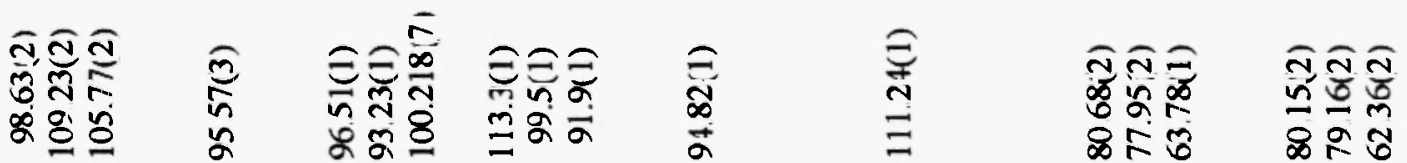

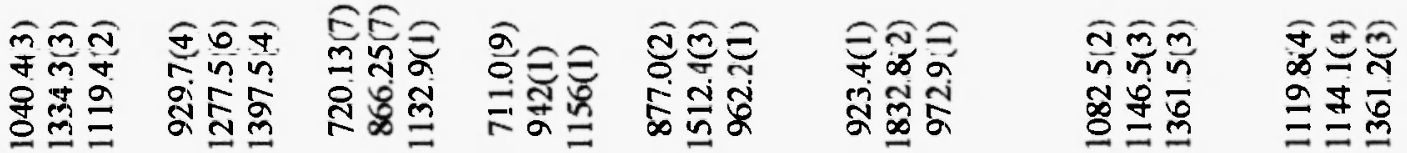

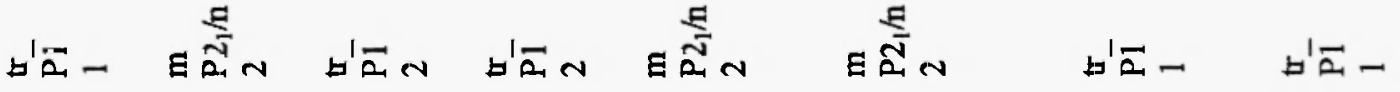

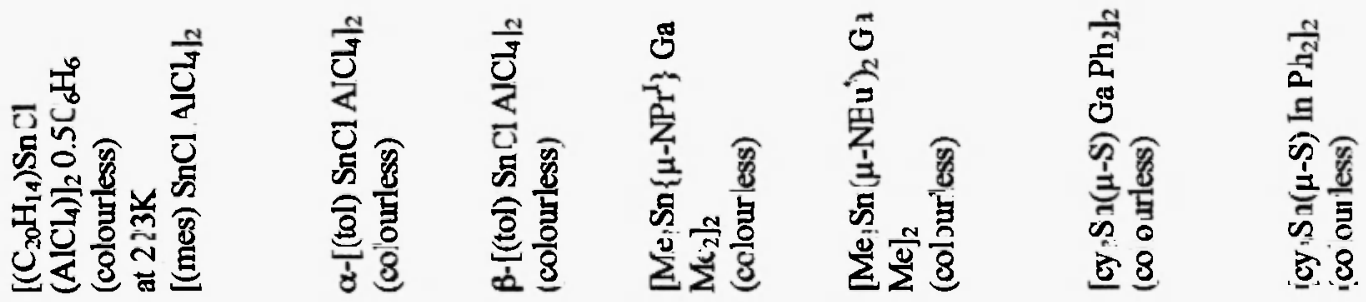




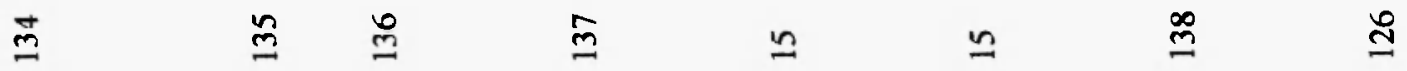

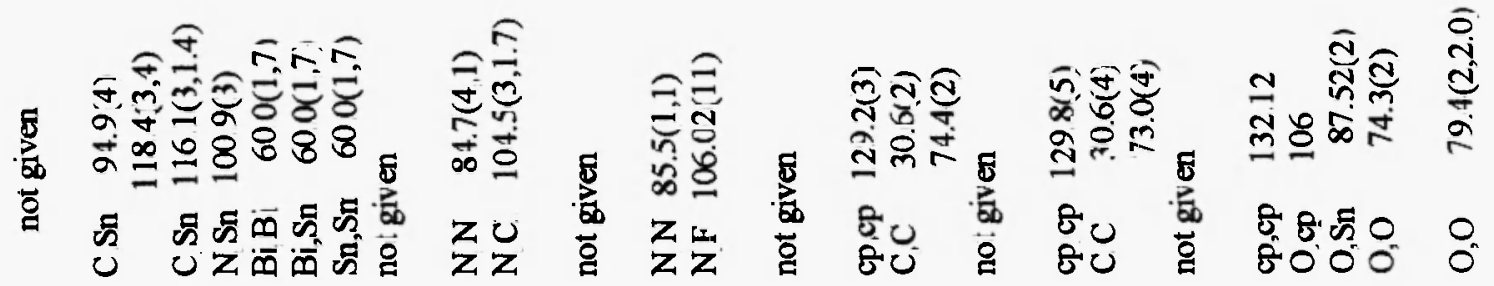

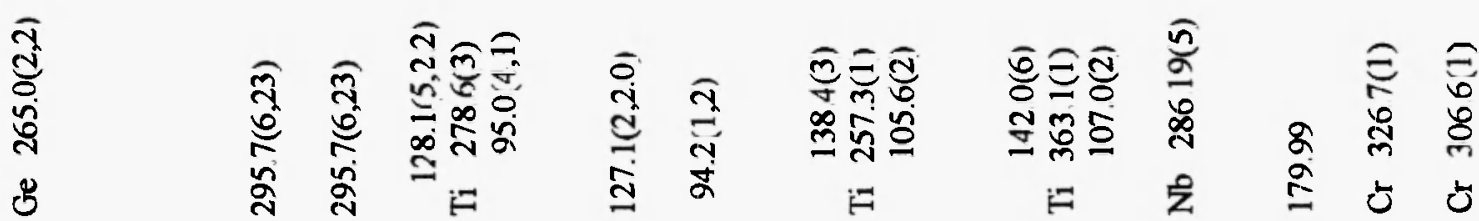

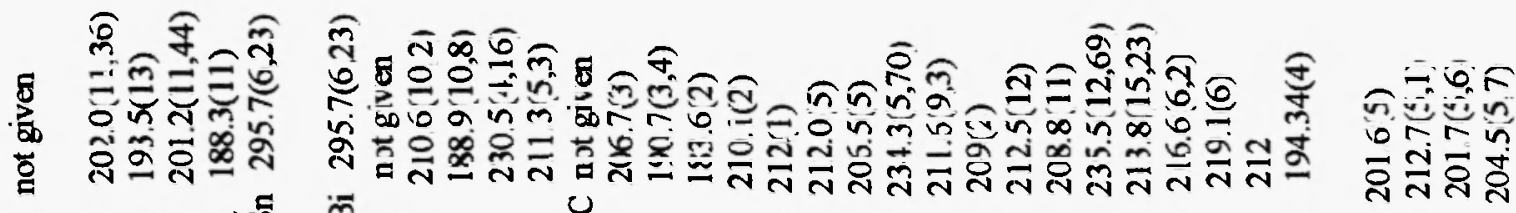

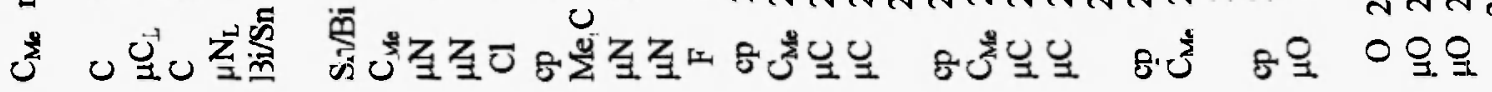

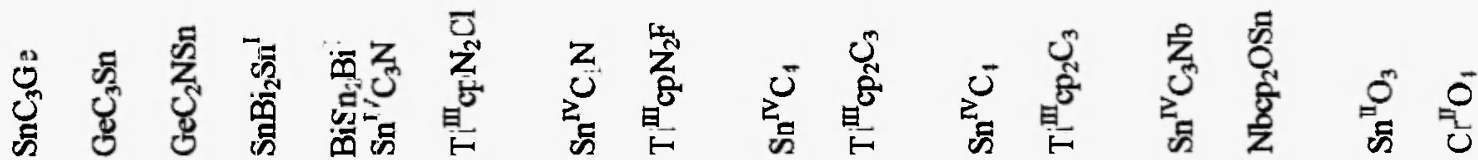<smiles>C=CC(C)C=C</smiles>

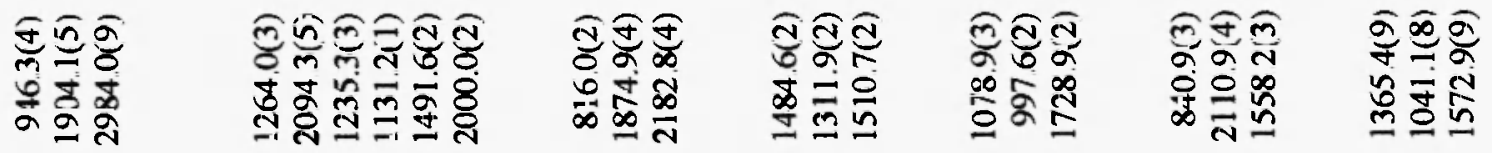

घ玄+

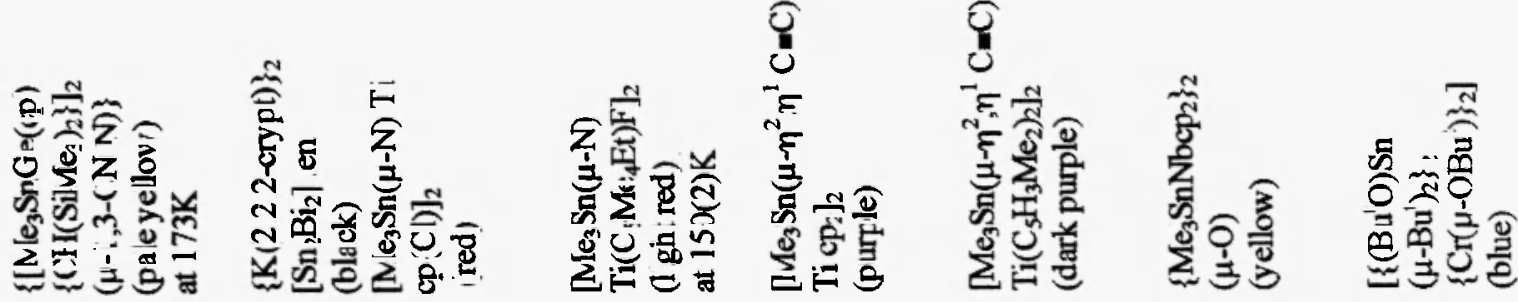




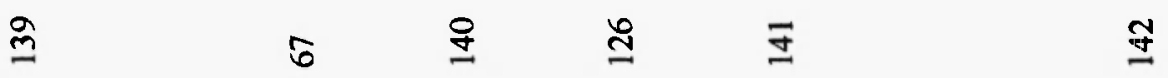

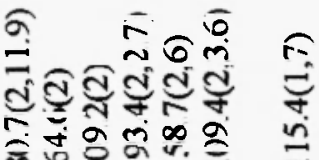

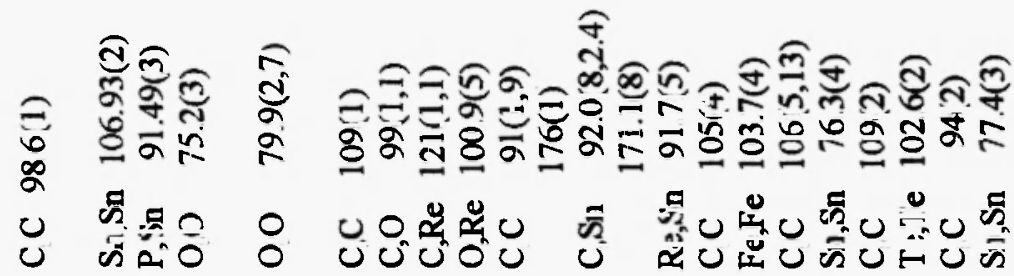

$\circ$ U U O 0 i

\begin{tabular}{|c|c|c|c|c|c|}
\hline 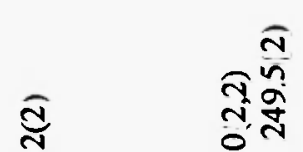 & 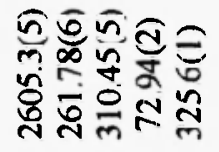 & $\begin{array}{l}\hat{\Xi} \\
\overline{8}\end{array}$ & $\frac{\bar{d}}{\stackrel{a}{d}}$ & $\begin{array}{l}\hat{\rho} \\
\text { o } \\
\text { }\end{array}$ & 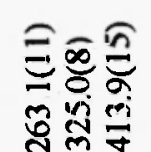 \\
\hline త్ & 豆 $\frac{1}{n^{5}}$ & 夏 & జ & $\mathscr{z}$ & 0 \\
\hline
\end{tabular}

FIำ

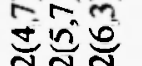

妾事的

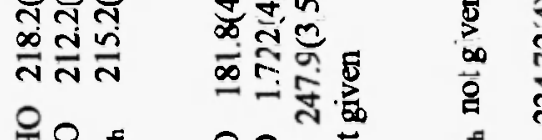

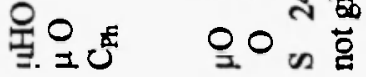

离
कू

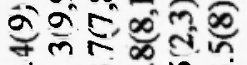

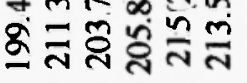

$\circ$ 일이 질

总

章

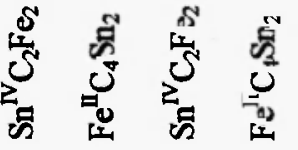

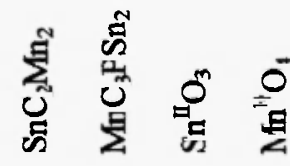

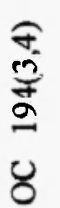

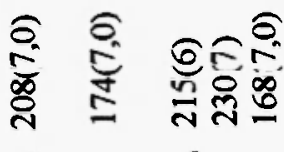

ठै 8 है 8

讷

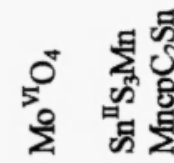<smiles>[B][CH]</smiles>

ิิ⺈)

$\infty \div 6$

总守苟

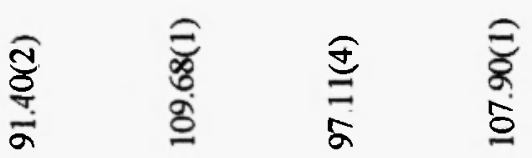

๖ั

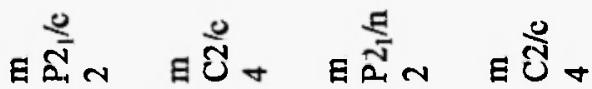
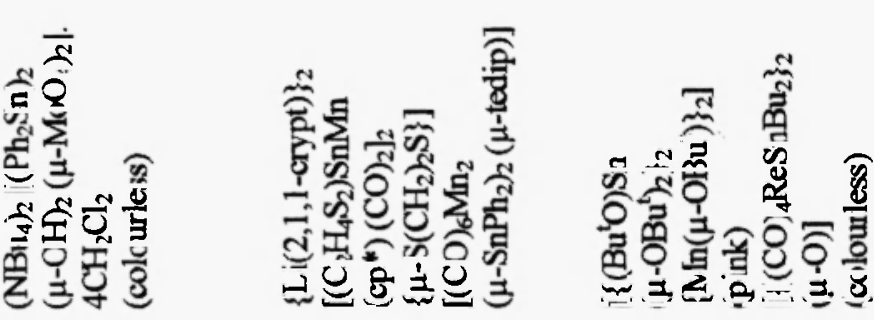

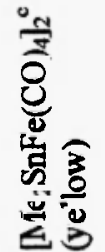

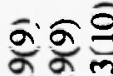

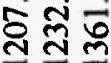

๖ 
罗 者

$\because$

过

包

$\stackrel{\infty}{ \pm}$

g

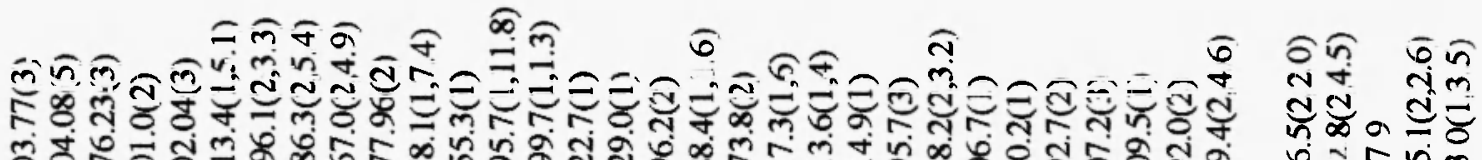

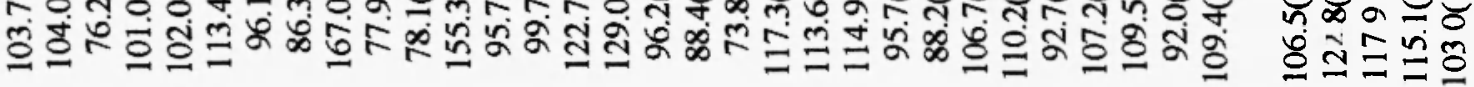

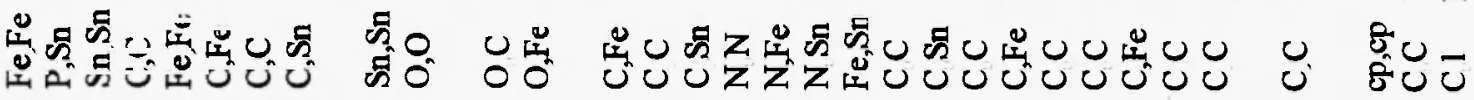

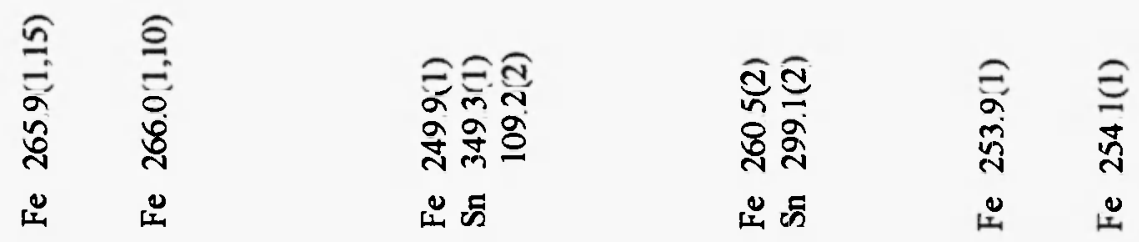

\begin{tabular}{|c|c|c|c|c|c|}
\hline 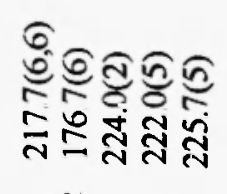 & $\begin{array}{l}\bar{a} \\
\overline{0} \\
\frac{1}{\infty}\end{array}$ & 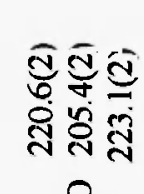 & 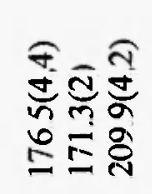 & 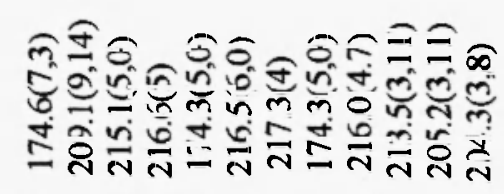 & ڤू' \\
\hline 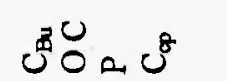 & 8 & & 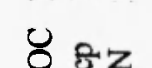 & ర ठ & \\
\hline
\end{tabular}

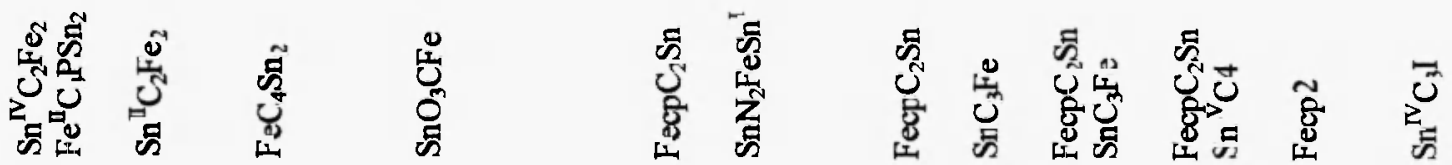

\begin{tabular}{|c|c|c|c|c|}
\hline 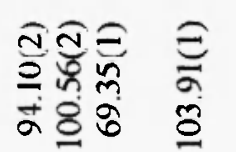 & 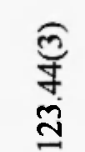 & $\begin{array}{c}\tilde{n} \\
\stackrel{\overbrace{}}{2} \\
\sigma\end{array}$ & 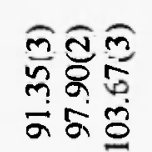 & 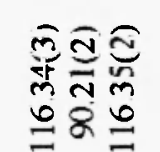 \\
\hline
\end{tabular}

\begin{tabular}{|c|c|c|c|c|}
\hline 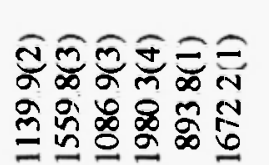 & 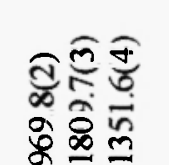 & 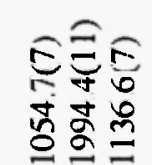 & 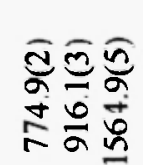 & 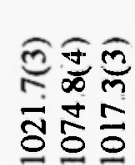 \\
\hline
\end{tabular}

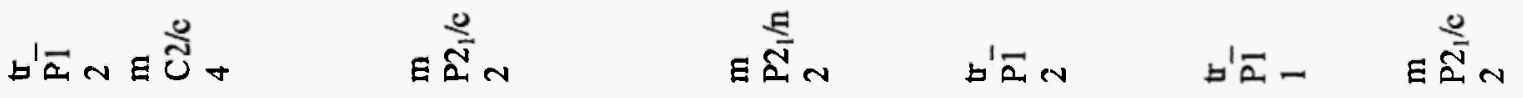
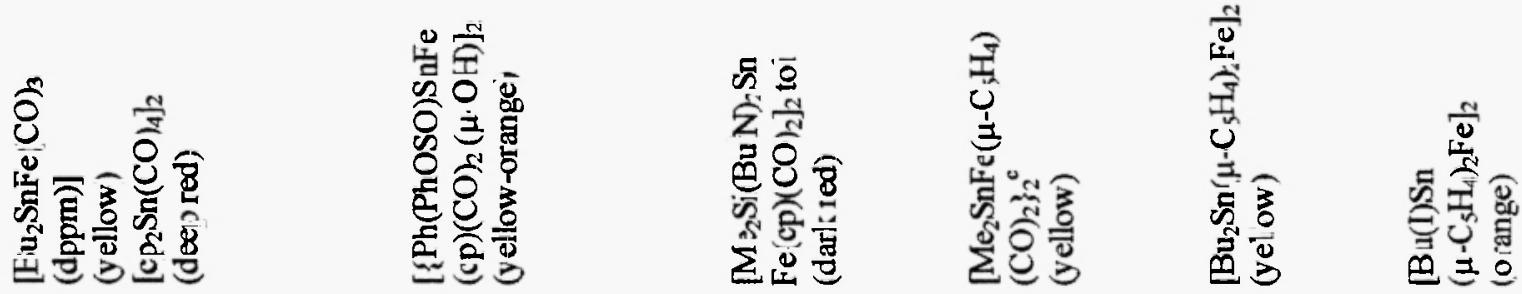


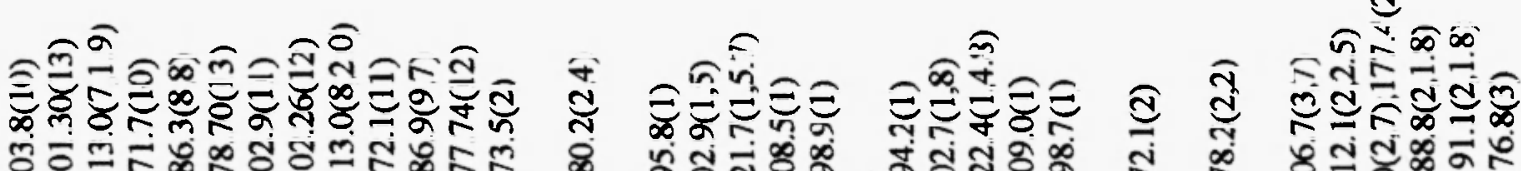

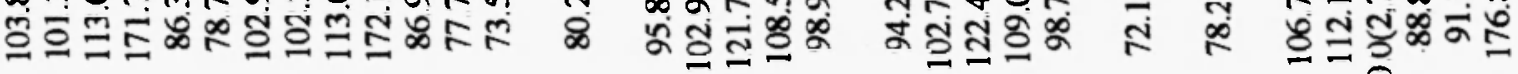

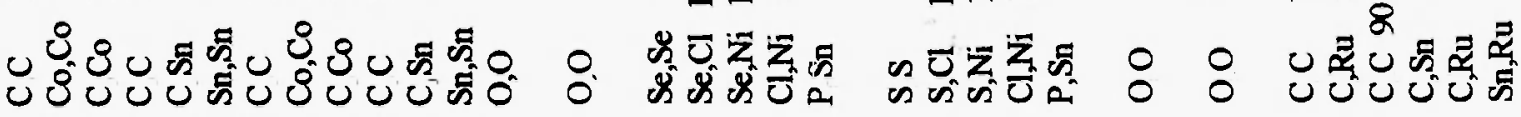

\begin{tabular}{|c|c|c|c|c|c|c|c|c|c|}
\hline 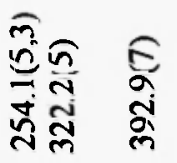 & 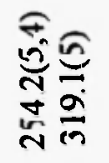 & 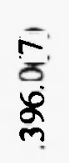 & 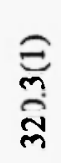 & 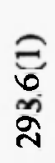 & 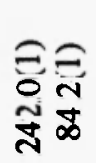 & 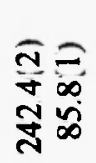 & 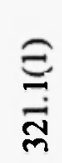 & 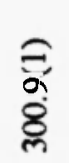 & 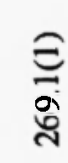 \\
\hline 8 मี & 8 मี & 8 & 8 & 8 & $\bar{z}$ & z & 乙 & 之 & $\not \vec{\not}$ \\
\hline
\end{tabular}

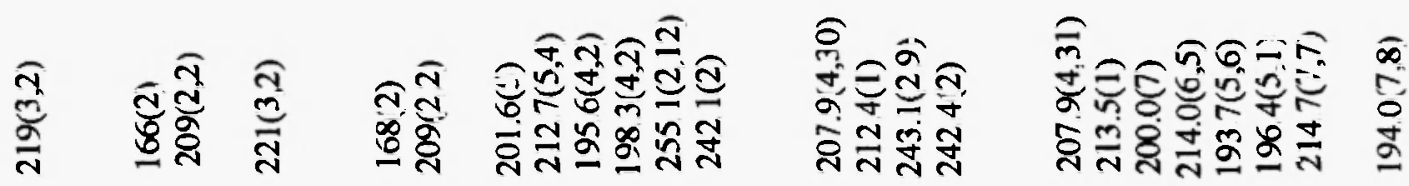
U

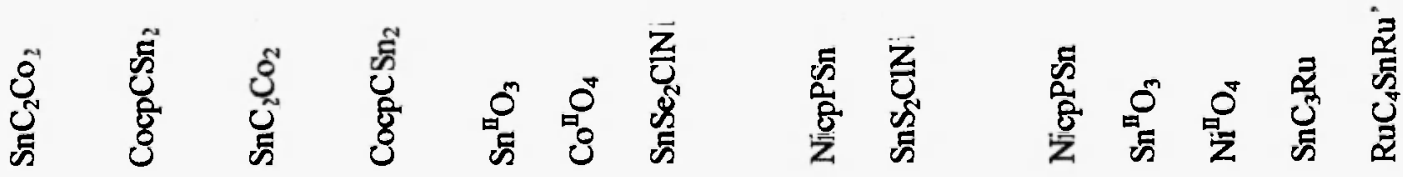

ิㅡํำ

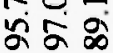

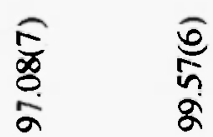

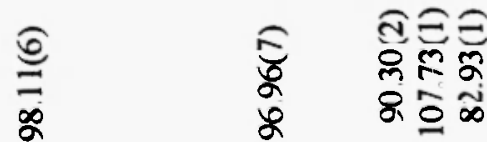

ิㅡㅇำ

크유

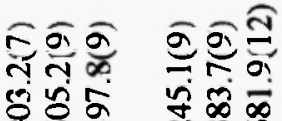

ํㅠㅀㅠ

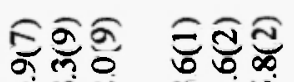

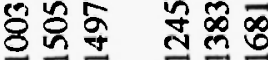

俩总总

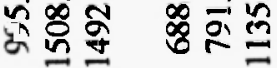

可

E $\stackrel{\frac{\pi}{2}}{\stackrel{\pi}{\pi}}$ E

$\pi \stackrel{\pi}{\check{N}}$

ะี

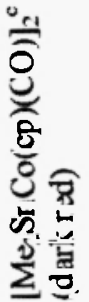

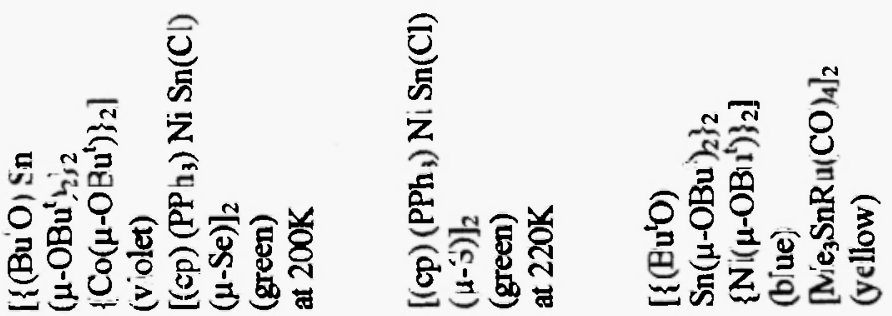




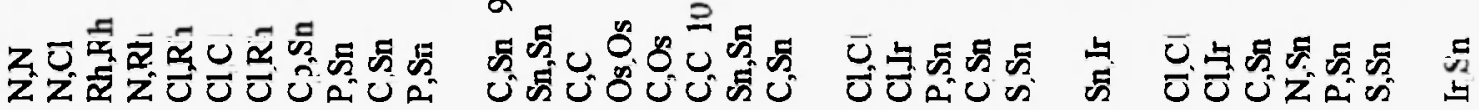

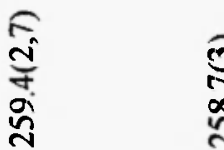

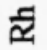

$\bar{\varrho} \cong$

สิ ปี

$z \bar{J}$

茎

क人

\% 过

m品

$\stackrel{\frac{\pi}{\pi}}{\frac{\Sigma}{2}}+$

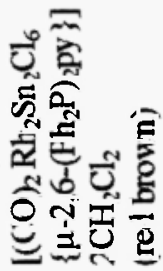

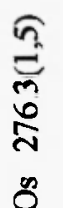

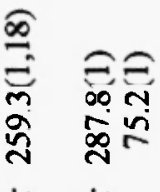

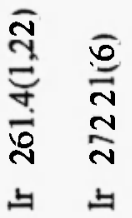

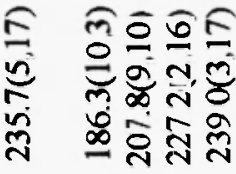

ஏ

a. ర్ర气<smiles>C=CC=C[Si]C</smiles>

氛芯

$\frac{\widehat{a}}{2}$

\section{氙突}

영

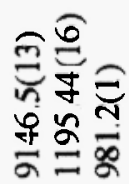

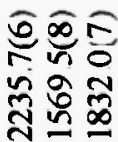

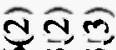

年

$\stackrel{\infty}{=} \stackrel{8}{=}$

E

घ

N
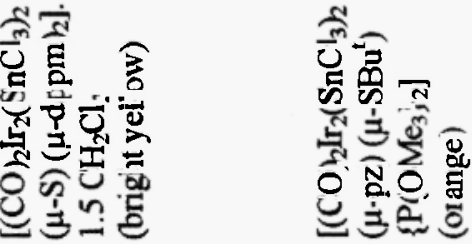
$\underline{n}$

$\stackrel{\infty}{2}$<smiles></smiles>

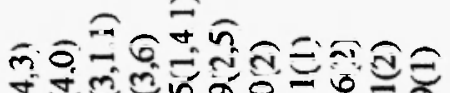

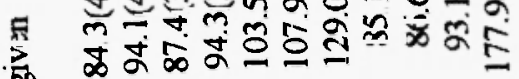

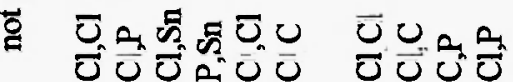

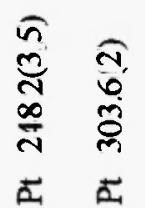

$\stackrel{\partial}{a}$

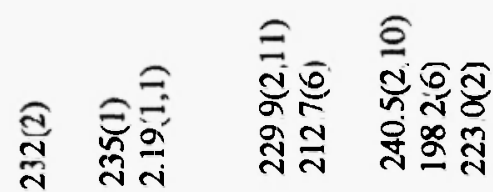

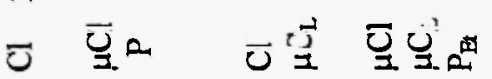

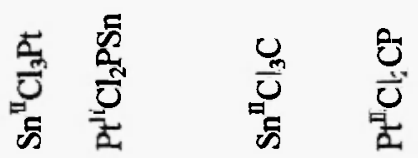

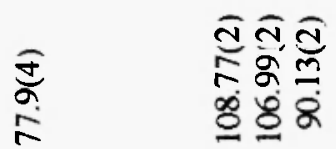

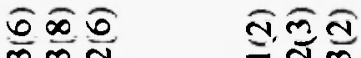

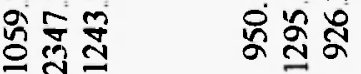

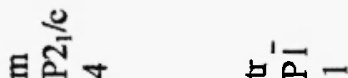
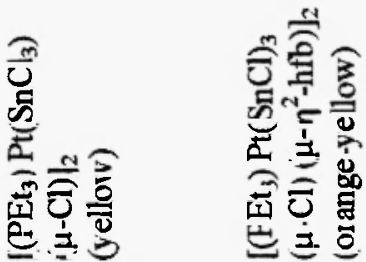

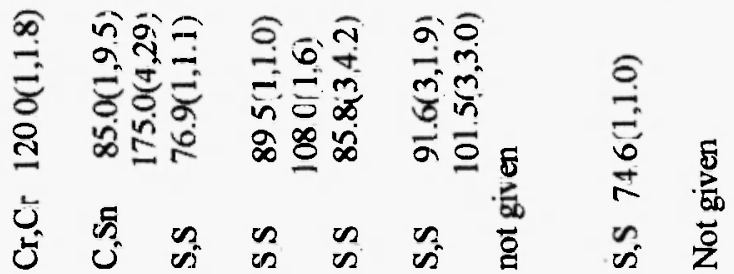

9 요
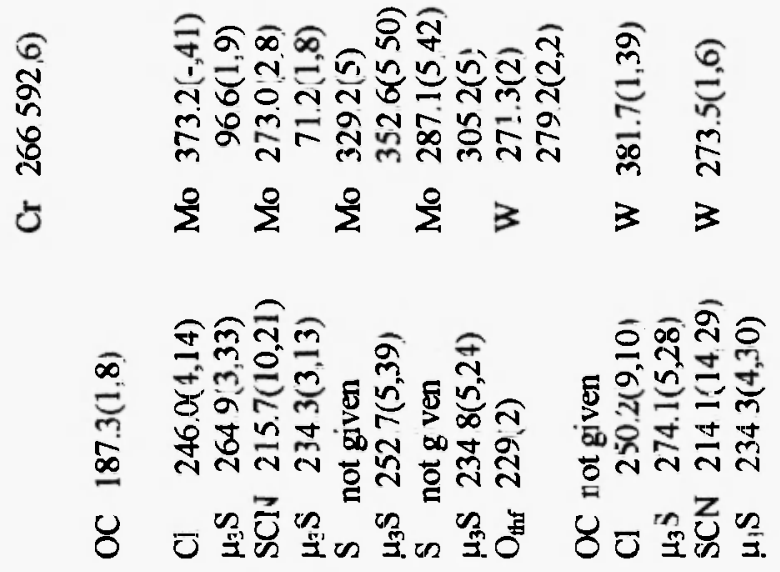

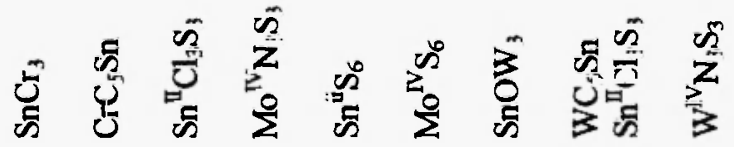

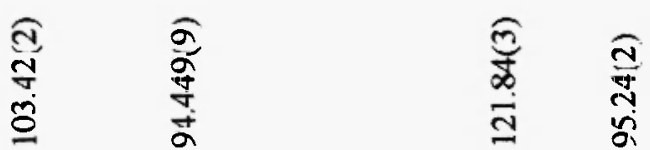

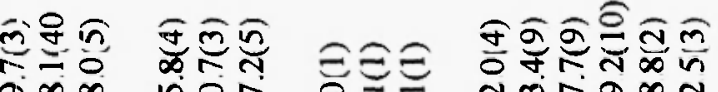

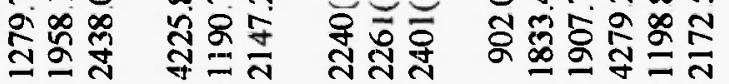

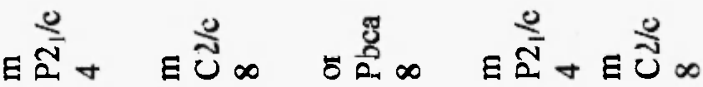

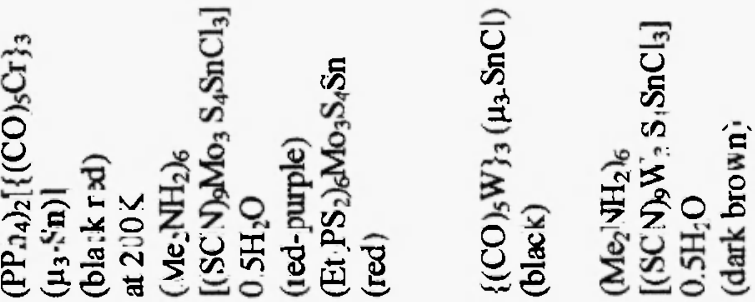


$\hat{\underline{3}}$

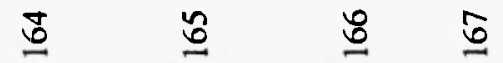

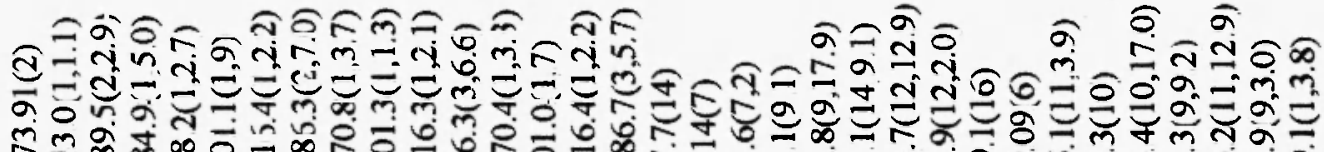

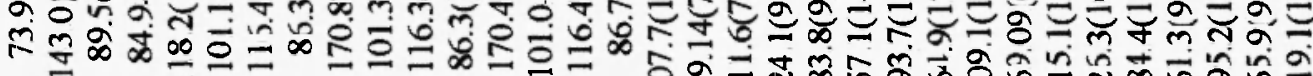

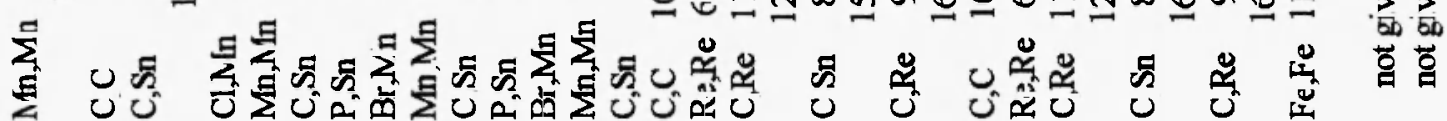

\begin{tabular}{|c|c|c|c|c|c|c|c|}
\hline 章 & $\frac{\overbrace{n}^{\infty}}{\stackrel{\infty}{(}}$ & 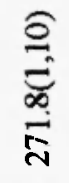 & 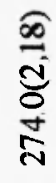 & 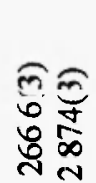 & 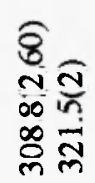 & 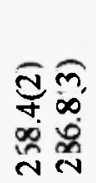 & 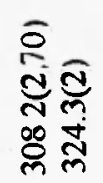 \\
\hline$\sum$ & 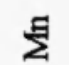 & $\sum^{5}$ & $\sum^{5}$ & "حّم & $\hat{\varkappa}$ & $\mathscr{2}$ & $\ddot{x}$ \\
\hline
\end{tabular}

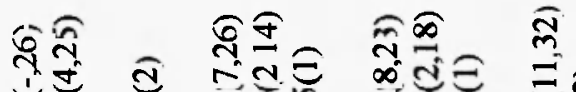

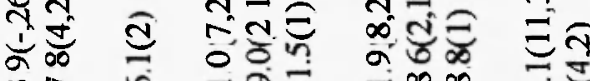

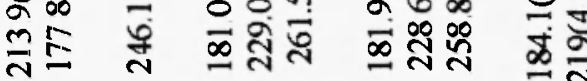

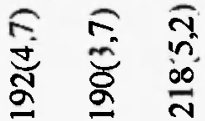

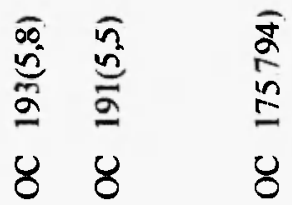

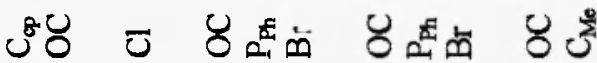

$8 \quad 8$ है

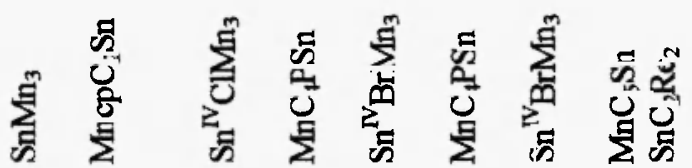

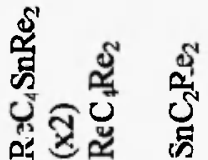

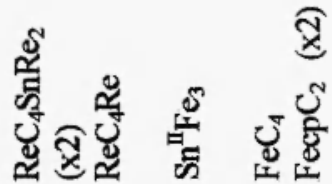

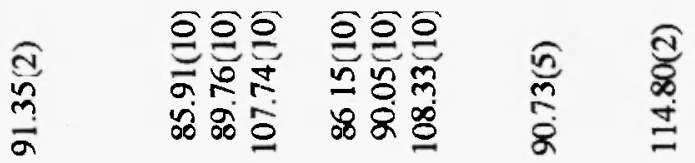

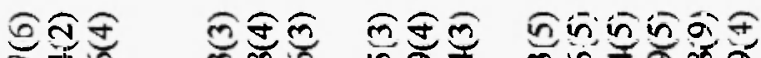

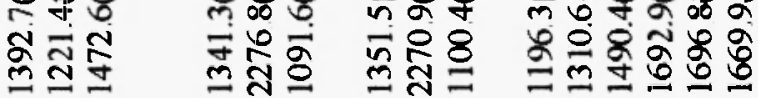

$\sqrt{n} \infty$

ธ่

Nㅡㅁ

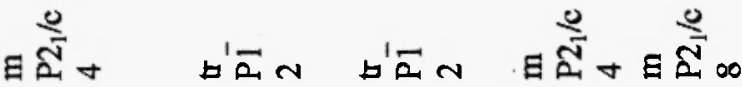

E $\stackrel{\text { ล }}{\text { E }}$
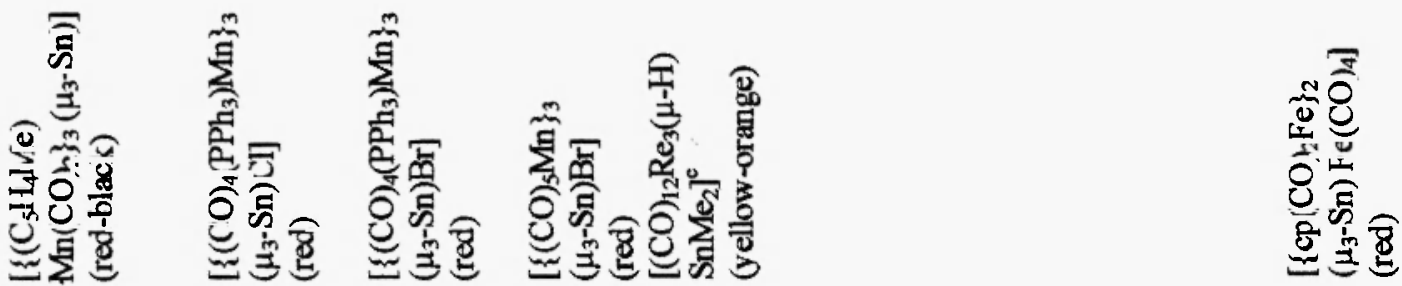
$\stackrel{0}{0}$

I 8 ณ

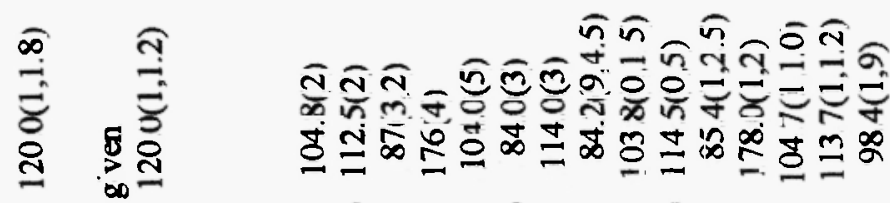

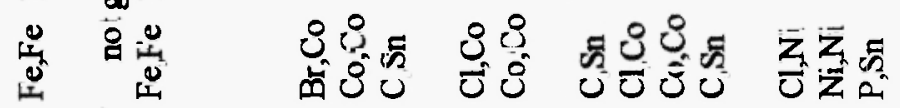

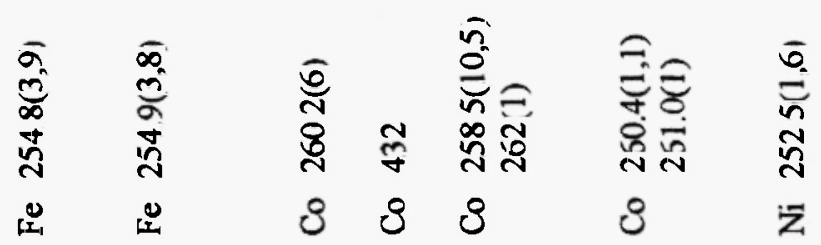

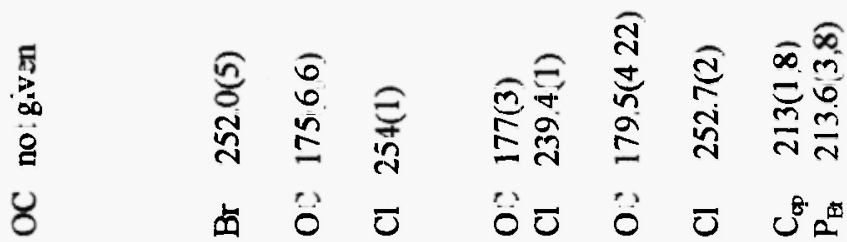

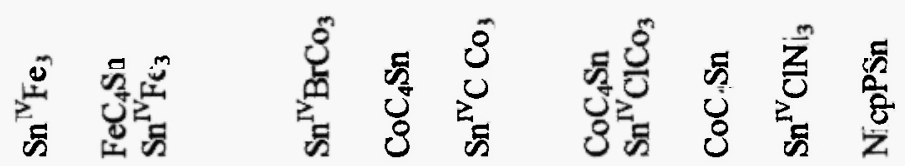

శ్ర్ర్⿻

$\hat{m}$
0
0

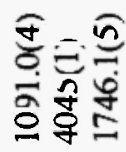

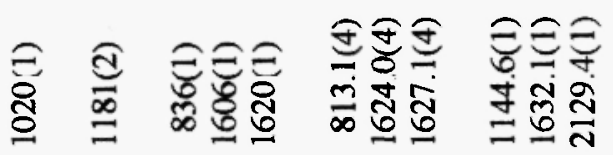

$\Xi \stackrel{\varrho}{\Sigma} \infty$

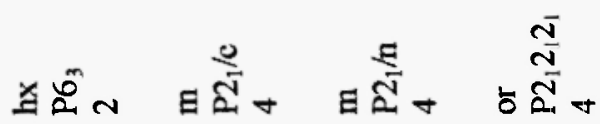
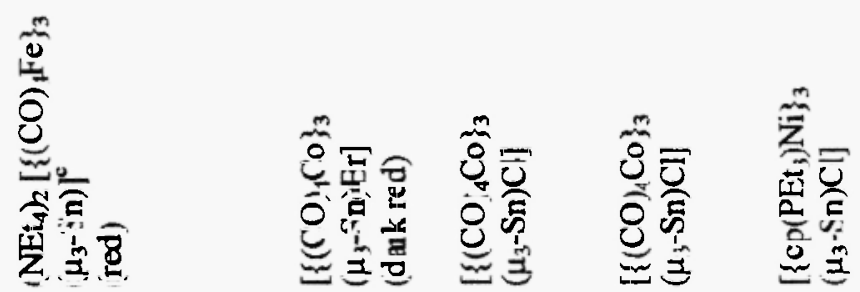


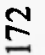

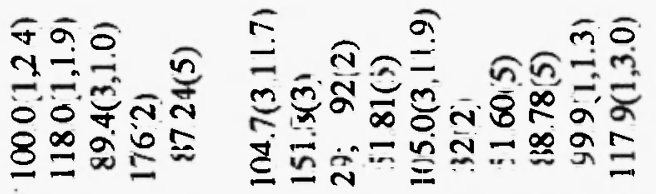

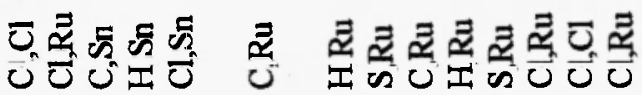

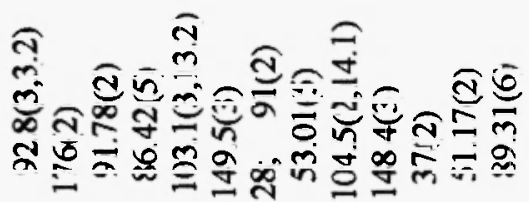

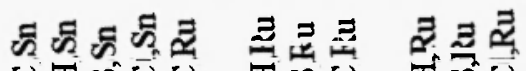

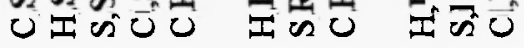

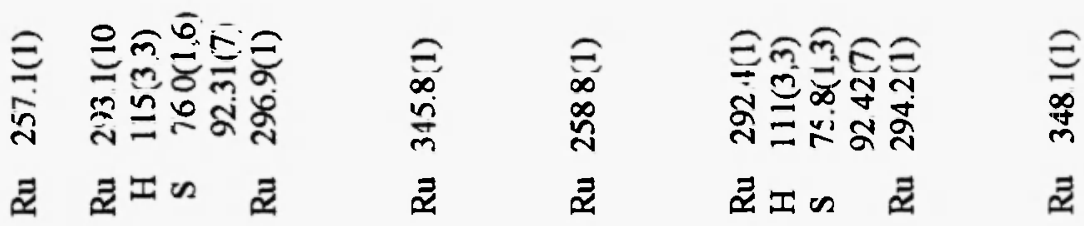

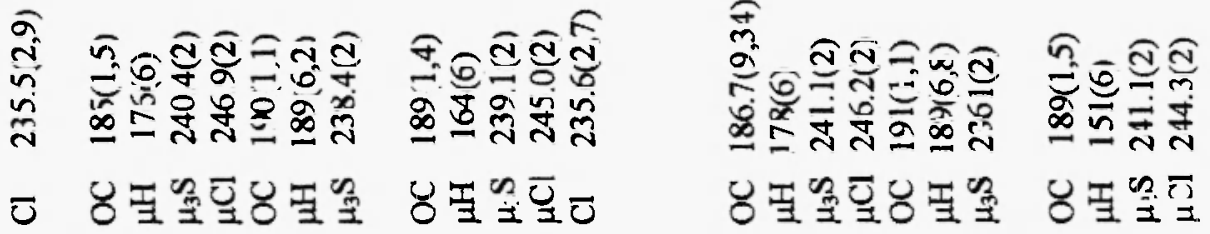

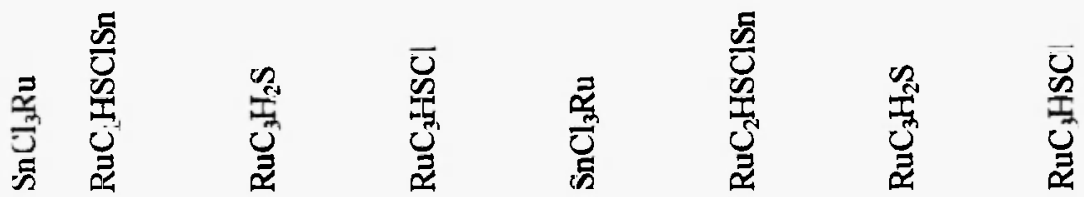

ำ

ㅎ⿱亠े

ิㅡำ

mo

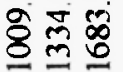

$b_{1} \bar{a}$

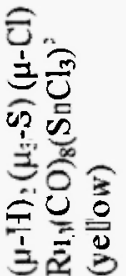


$\stackrel{m}{I}$

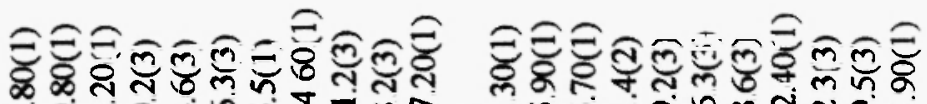

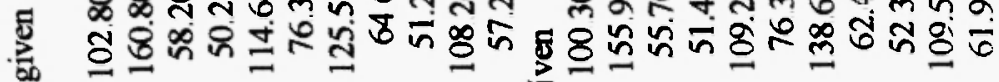

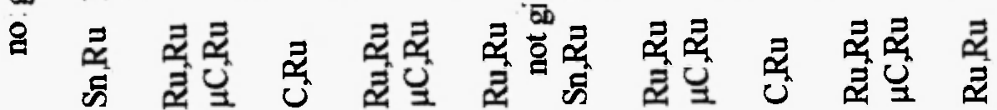

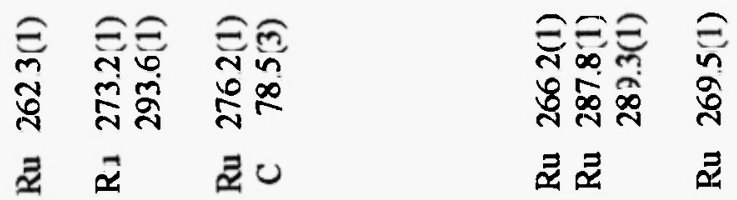

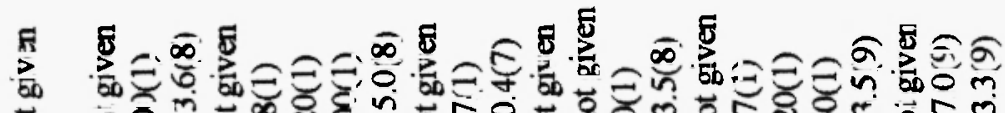

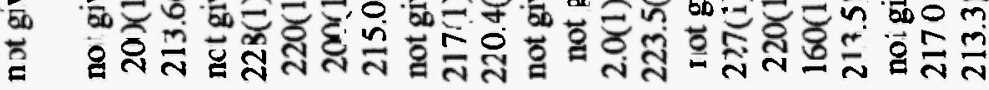

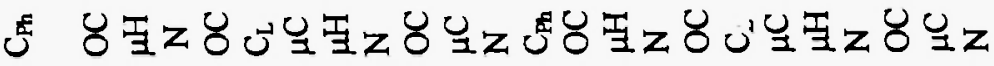

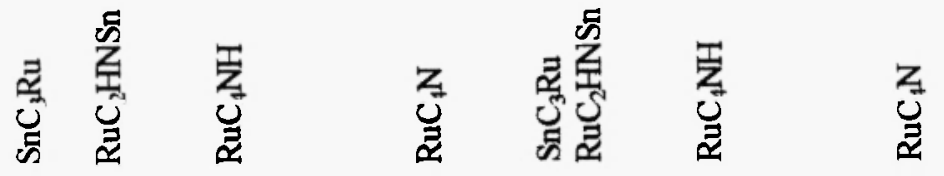

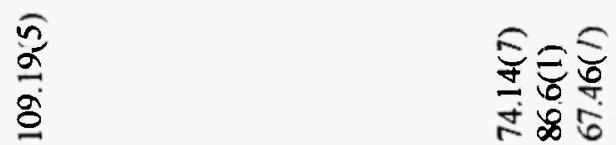

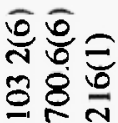

Eิ응

它电

$\frac{a}{\stackrel{2}{\Sigma}}+$

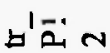

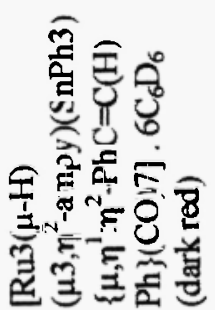

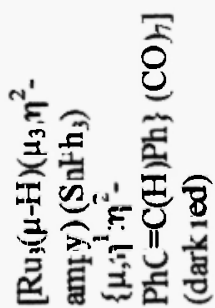


๙

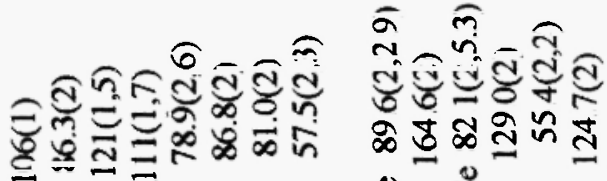

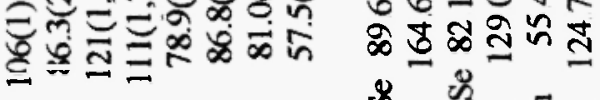

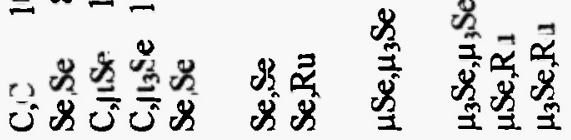

\begin{tabular}{|c|c|}
\hline 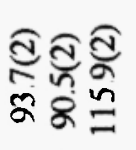 & 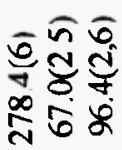 \\
\hline 总总 & $\vec{z}$ \\
\hline
\end{tabular}

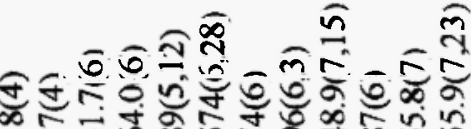

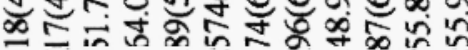

त̄

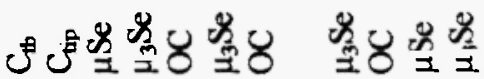

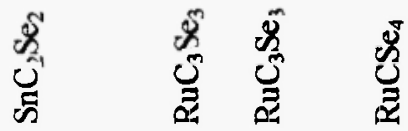

$\widehat{\widetilde{\gamma}} \overparen{ِ}$

怘各す

ชิธิู

$\infty m$ n

กำ

ำำ응

$b^{\prime} \vec{a}$

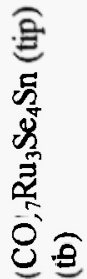


$\stackrel{n}{\Sigma}$

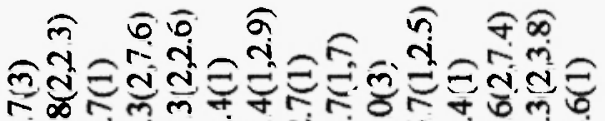

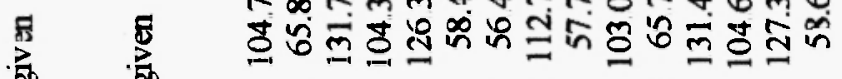

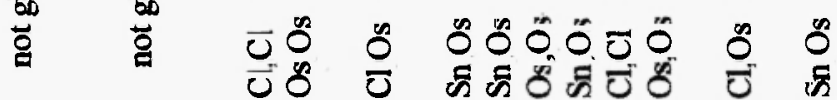

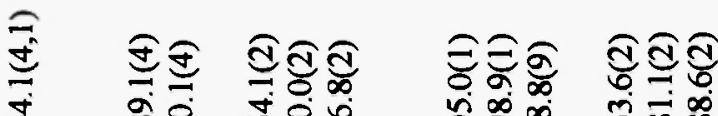

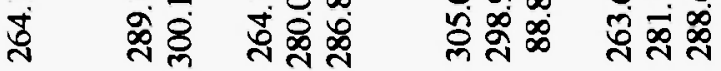

88

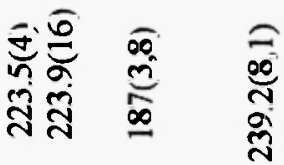

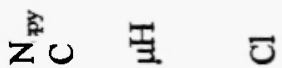

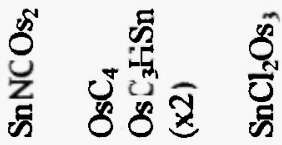

क्ञ

高

$\frac{\pi}{3}$

कิธชช

ริธู่ส

ติำ

递苍

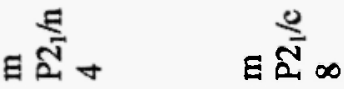

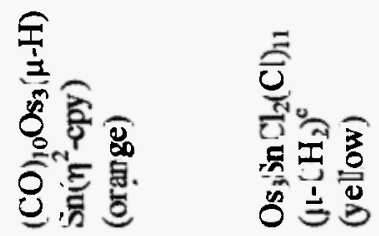

888

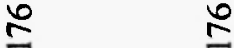

ส

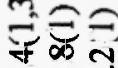

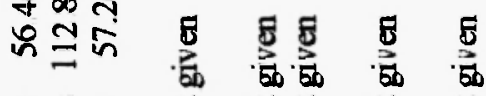

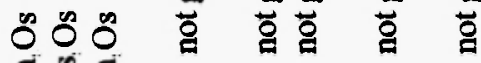

सै 8 है

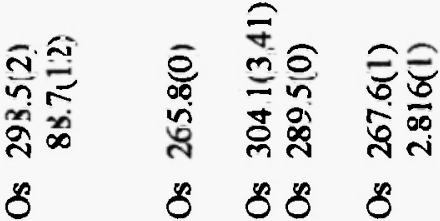

$\underset{\substack{i \\ i}}{i}$

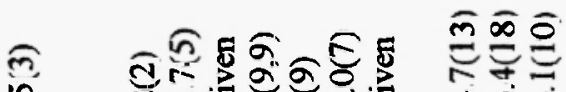

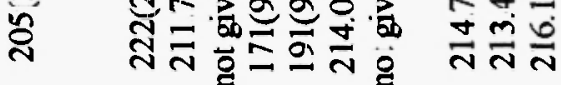

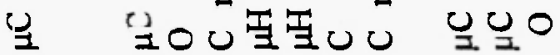

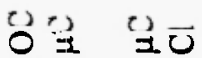

8

号

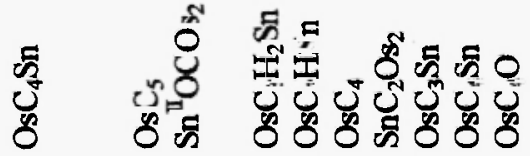


$\Xi \quad \stackrel{\infty}{\Xi}$

a

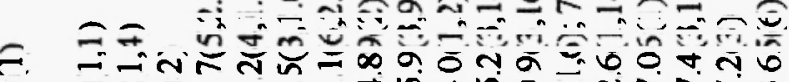

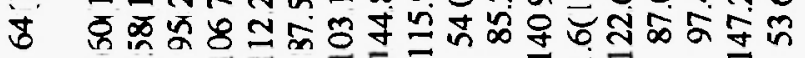

क्ष

\begin{tabular}{|c|c|c|c|c|}
\hline 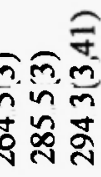 & 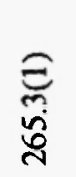 & 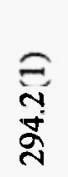 & 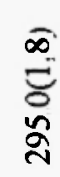 & 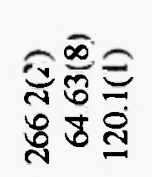 \\
\hline 8 & 8 & 8 & 8 & 8 \\
\hline
\end{tabular}

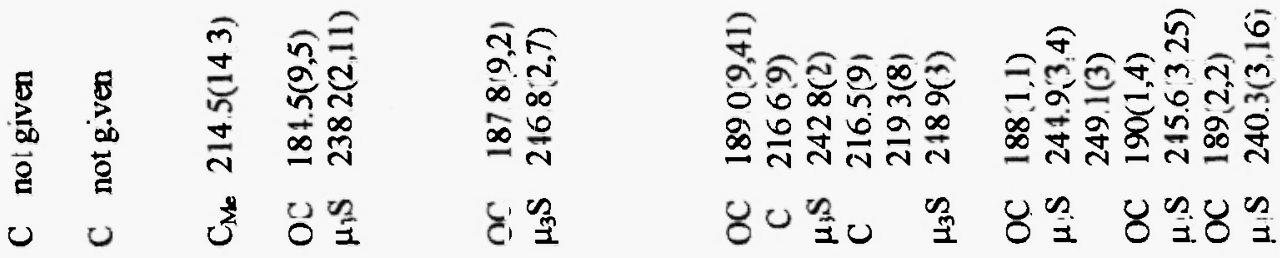

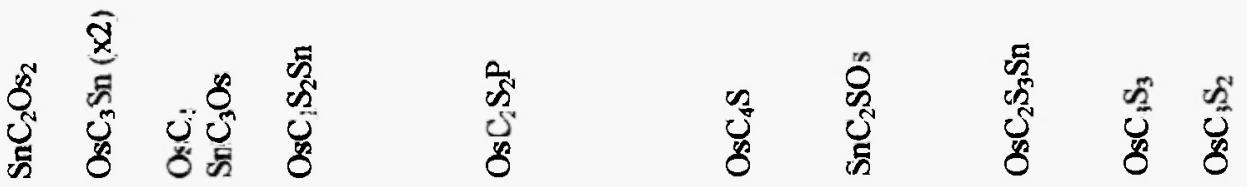

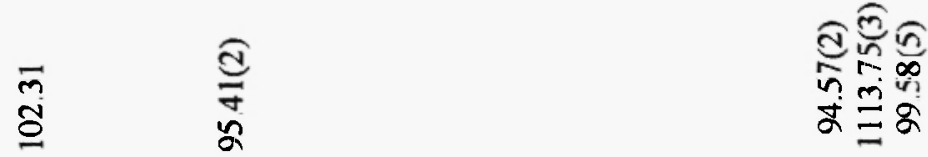

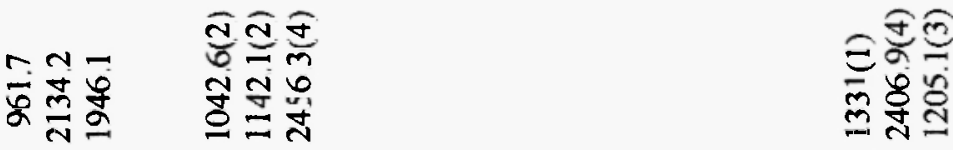
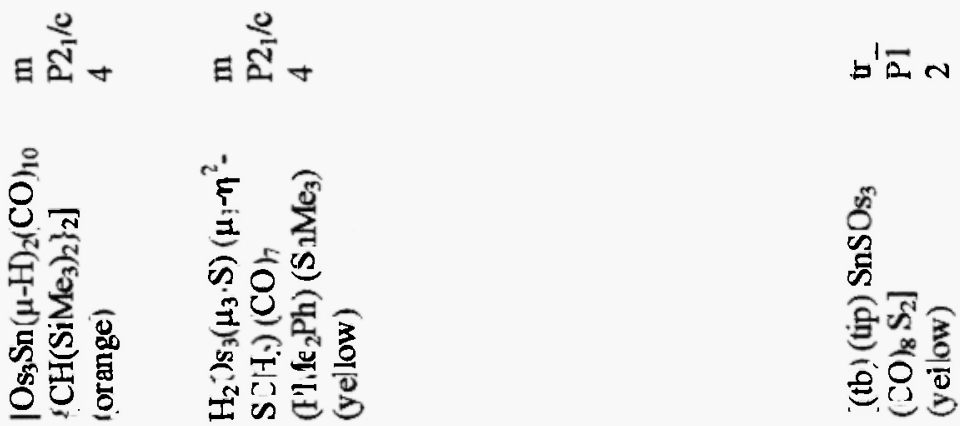
a $\stackrel{\infty}{\infty} \stackrel{\infty}{=}$

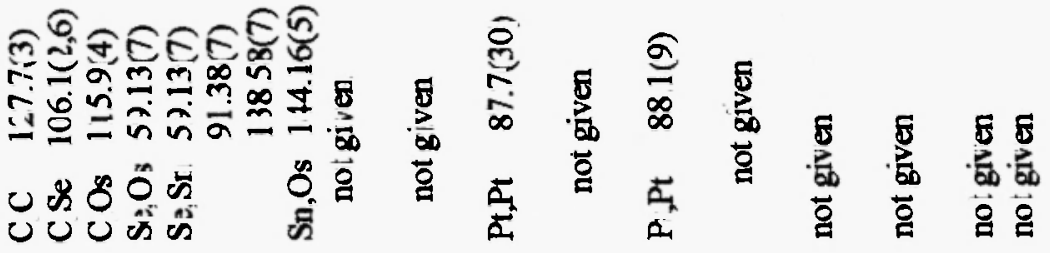

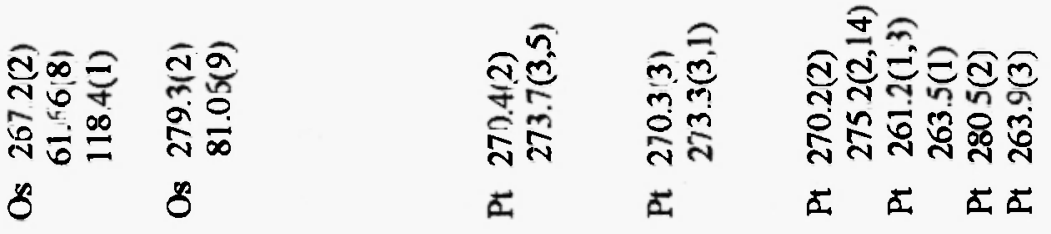

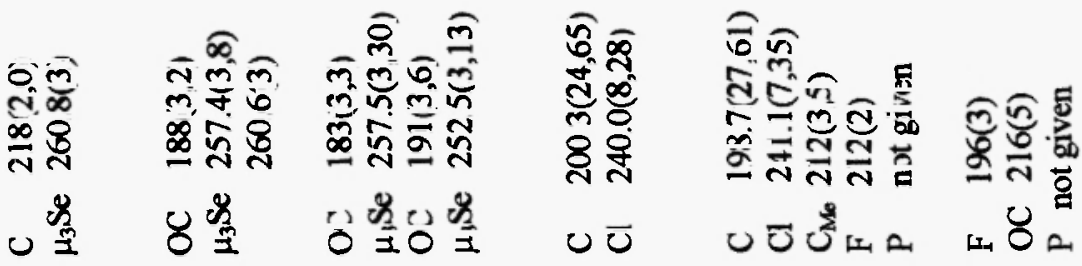

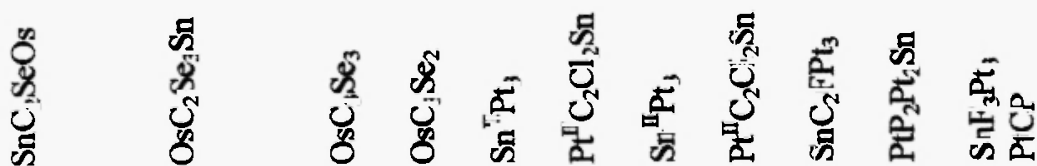

ติำ

충도

\&oำ

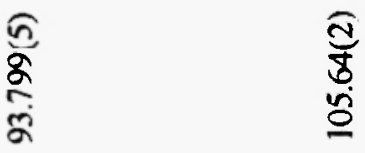

突氙

ลิEิ

8 \%

cio

$\infty \infty$

$\subseteq \infty 9$

䋉学

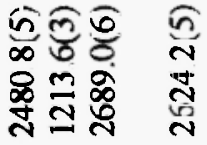

$\forall \operatorname{As} N$

$E 8 \infty$

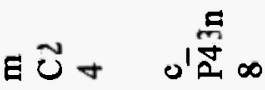
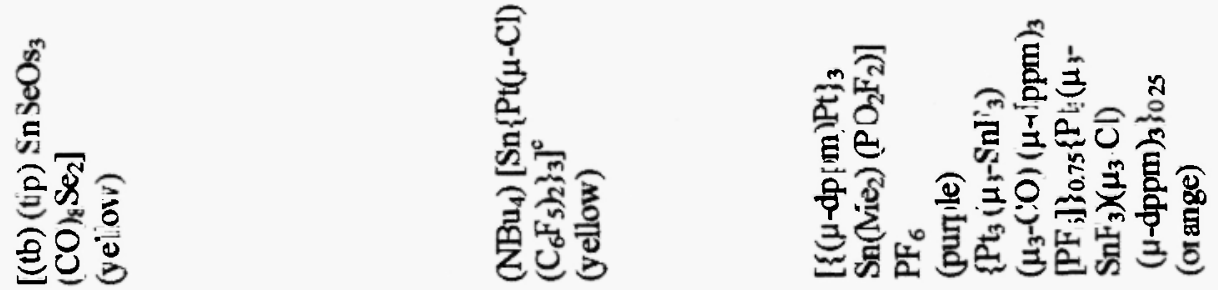
$\underset{0}{\infty}$

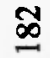

$\stackrel{\infty}{\infty}$

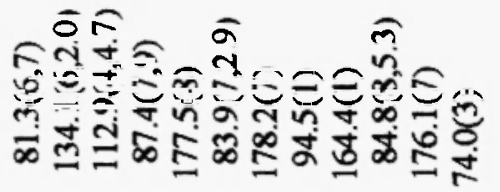

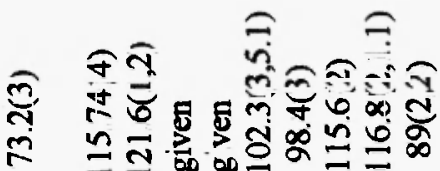

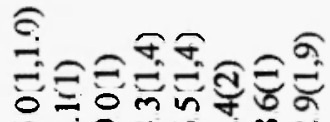

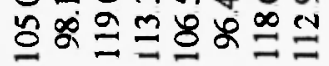

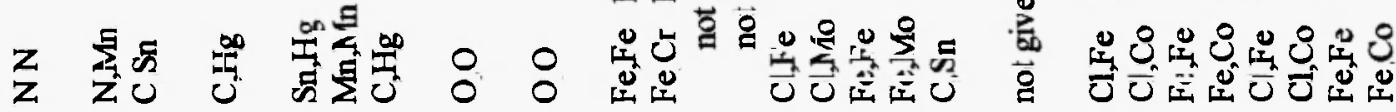

层 0

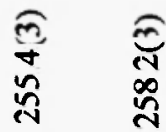

兵 些

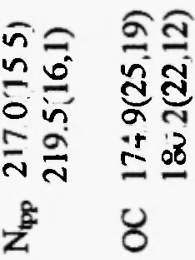

$\sum_{\text {Z }}^{\text {E }}$

สิธิต

m $m$

종

অํํㅇ

㐫㐫势

再每

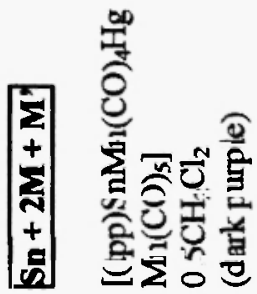

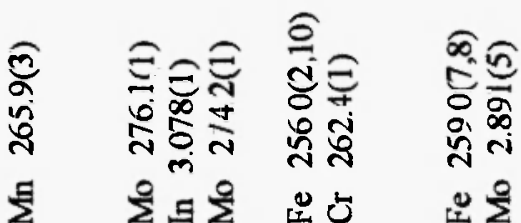

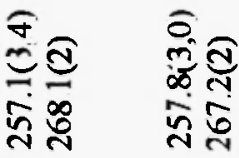

\& 8 \&

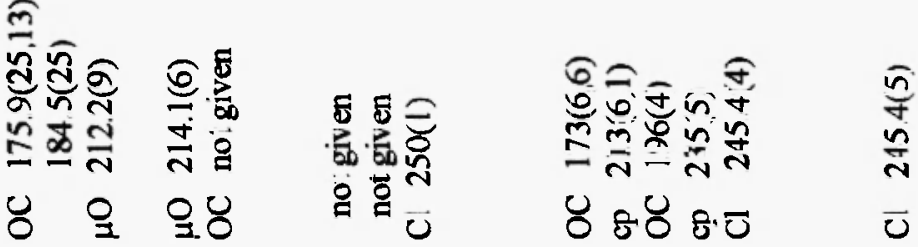

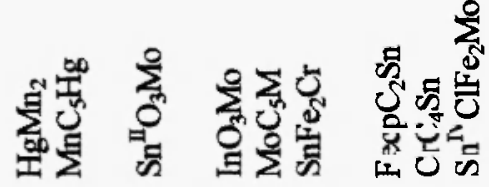

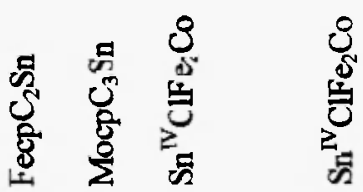

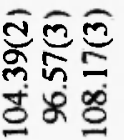

\&̊

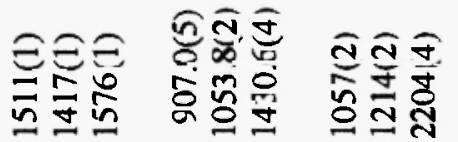

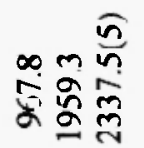

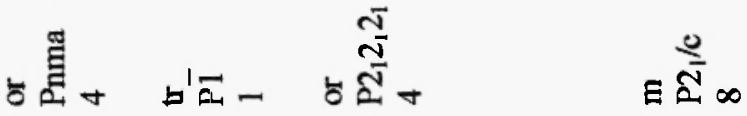

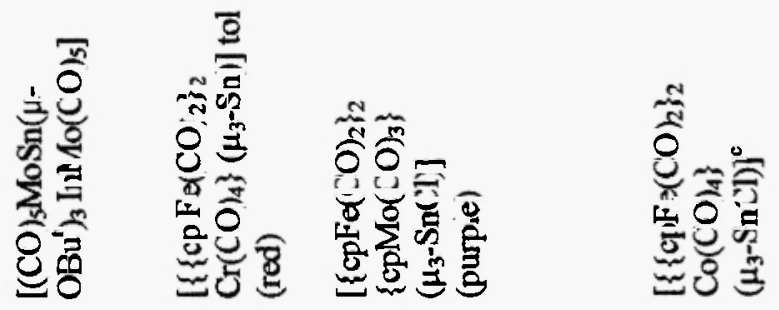




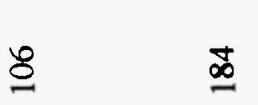

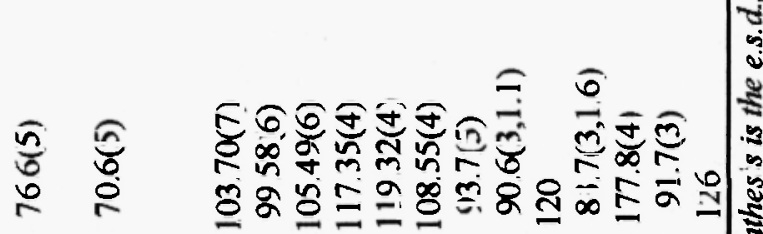

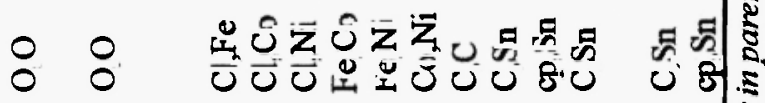

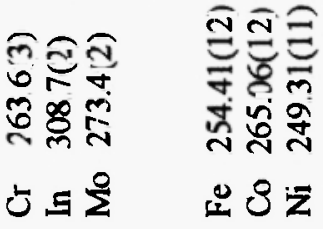

)

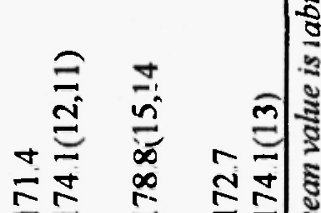

ЮЧరठ

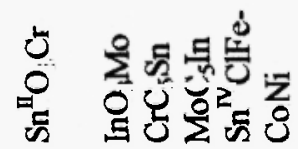

8ठ

폰

I

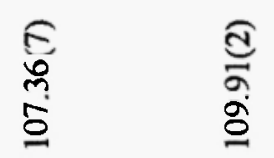

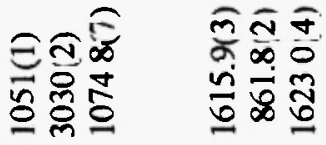

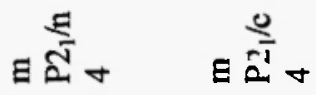

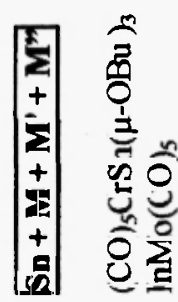

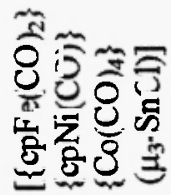


bonds. 274.2(1) pm (Mo-In), 276.1(1) pm (Mo-Sn), and 307.8(1) pm (Sn-In).

In a red cluster [168] two cpFe(CO) 2 units and a $\mathrm{Cr}(\mathrm{CO})_{4}$ unit are directly bonded to $\mathrm{Sn}$ with $\mathrm{Fe}$-Sn bond distances of 255.0(2) and 257.0(1) pm, and Sn-Cr distance of 262.4(1) pm. The Fe-Sn-Fe bond angle is $115.74(4)^{\circ}$, and the mean $\mathrm{Fe}-\mathrm{Sn}-\mathrm{Cr}$ angle is $121.6(1)^{\circ}$.

A purple SnFe2Mo cluster [182] has a tin atom in the central position bonded to two $\mathrm{cpFe}(\mathrm{CO})_{2}$ and one cpMo(CO) 3 moities. The Sn-Fe bond distances are 259.3(7) and 259.8(7) pm, and a Sn-Mo distance of 289.1(5) pm. The relevant bond angles are 115.6(1) ${ }^{\circ}$ (Fe-Sn-Fe) and 116.8(2) (Fe-Sn-Mo, mean). A CI atom on $\mathrm{Sn}$ (IV) completes the a distorted tetrahedral arrangement ( $\mathrm{SnClFe} 2 \mathrm{Mo}$ ). A related cluster $\mathrm{SnFe}_{2} \mathrm{Co}_{\text {[183] again }}$ has all three moities, two $\mathrm{cpFe}(\mathrm{CO})_{2}$ and one $\left.\mathrm{Co}(\mathrm{CO})_{4}\right)$ bound directly to $\mathrm{Sn}(\mathrm{IV})$. A CI atom completes the distorted tetrahedral environment of the tin $\left(\mathrm{SnClFe}_{2} \mathrm{Co}\right)$. The structure contains two crystallographically independent clusters differing mostly by degree of distortion.

\subsection{Sn plus $M$ and $M^{\prime}$ and $M^{\prime \prime}$ \\ Two clusters contain four different metal atoms are summarised in Table 2. The first [106]}

contains a (CO)sCr-Sn( $\mu$-OBu ) 3 In-Mo(CO)s array, with $\mathrm{Cr}$-Sn, Sn-In and In-Mo distances of 263.693), 308.7(2) and 273.4(2) pm, respectively. Only the first and the last represent actual metal-metal bonds.

In a SnFeCoNi cluster [184] a central Sn(IV) atom has an almost perfect tetrahedral geometry even though bonded to three different moities, $\mathrm{cpFe}(\mathrm{CO})_{2}, \mathrm{cpNi}(\mathrm{CO})$ and $\mathrm{Co}(\mathrm{CO})_{4}$, and a terminal $\mathrm{CI}$ atom. The Sn$M$ bond distances increase in the order: $249.31(11) \mathrm{pm}(\mathrm{Sn}-\mathrm{Ni})<254.41912) \mathrm{pm}(\mathrm{Sn}-\mathrm{Fe})<265.61(11) \mathrm{pm}(\mathrm{Sn}-$ $\mathrm{Co})$. The M-Sn-M' bond angles open in the order: $108.55(4)^{\circ}(\mathrm{Co}-\mathrm{Sn}-\mathrm{Ni})<117.35(4)^{\circ}(\mathrm{Co}-\mathrm{Sn}-\mathrm{Fe})<119.32(4)^{\circ}$ (Ni-Sn-Fe).

The data in Table 2 shows atoms in oxidation states $0,+2$ and +4 , but there are several clusters without distinguishable oxidation states assigned to the metal atoms. The tin atoms are three, four, five and six coordinate, with distorted tetrahedral being the most common. The most common C-donor ligand is the methyl group, usually with a CI atom co-donor. However, in the three coordinate Sn(II) derivatives, the most common donor atom is $\mathrm{O}$ (usually from the $\mathrm{OBu}$ moiety). In these latter cases the $\mathrm{Sn}$ (II)- $\mathrm{L}$ bond distances are longer for $\mathrm{N}$-donors than O-donors. the values being $217.5 \mathrm{pm}$ versus $203 \mathrm{pm}$ for terminal ligands and $226 \mathrm{pm}$ versus 211 pm for bridging ligands, respectively. The mean Sn(II)-L versus Sn(IV)-L bond distances for tetrahedrally coordinated species are: 217 versus $216.5 \mathrm{pm}(\mathrm{CL})$ and 248 versus $237 \mathrm{pm}$ (CI).

About $18 \%$ of the heterotetrameric tin compounds contain non-transition elements. A summary of the non-transition $\mathrm{Sn}-\mathrm{M}$ and $\mathrm{M}-\mathrm{M}$ distances are given in Table $2 \mathrm{~A}$. Only three derivative to $\mathrm{Hg}$, $\mathrm{Ge}$ or $\mathrm{Bi}$ can be considered to exhibit a metal-metal bond. The remainder of the examples (about $80 \%$ ) contain transition metals in clusters with tin, a rich and complex chemistry. A summary of these $\mathrm{Sn}-\mathrm{M}$ and $\mathrm{M}-\mathrm{M}$ distances is given in Table 2B. The shortest bond is the Sn-Ni bond at 242.0(1) pm [151]. The mean Sn-M bond distance increases with the covalent radius of the heterometal atom, with the exceptions of Pd, Rh and Ir. The length of the Sn-M bond varies markedly with the electronegativity of the groups attached to the $\mathrm{Sn}$ atom, as does the deviation from tetrahedral shape. The shortest Sn-M bond distances are found in the heterotrimeric clusters (Table 1A), except for three cases. These are: 264.8(1) pm (tri-) versus $253 \mathrm{pm}$ (tetra-) for Sn-Os; 258.5(2) versus 251.7(1) pm for Sn-Pd; and 273.6(1) pm versus $266.6(3)$ pm for Sn-Re. The mean Sn-M distances are also generally shorter in the trimeric than the tetrameric derivatives, but again there are exceptions. These are: $266.0 \mathrm{pm}$ (tri) versus $262.8 \mathrm{pm}$ (tetra) for $\mathrm{Sn}-\mathrm{Ru}, 259.8 \mathrm{pm}$ versus $253.0 \mathrm{pm}$ for $\mathrm{Sn}-\mathrm{Pd}$; $264.5 \mathrm{pm}$ versus $259.2 \mathrm{pm}$ for $\mathrm{Sn}$-Ir, and $280.8 \mathrm{pm}$ versus $278.6 \mathrm{pm}$ for Sn-Mn. One example [130] is found in two isomeric forms. Several examples $[114,117,124,142,147,150,167,169,172,179,183]$ contain two crystallographically independent molecules which represent examples of distortion isomerism [113].

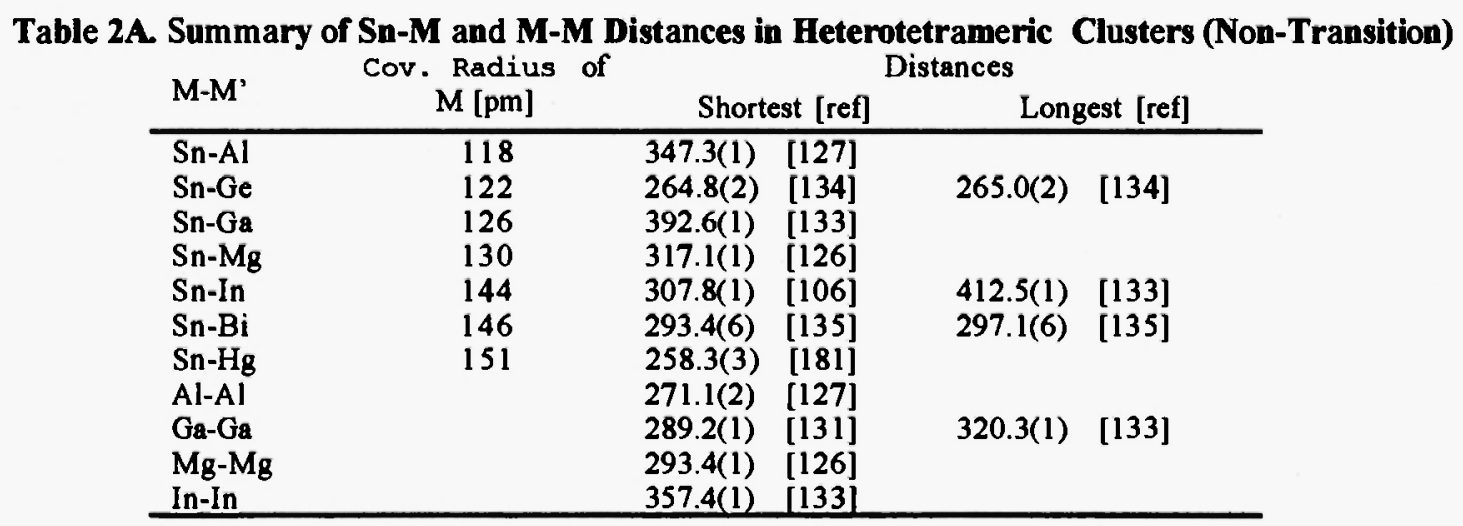


Table 2B. Summary of Sn-M and M-M distances in Heterotetrameric Clusters (Transition)

\begin{tabular}{|c|c|c|c|c|c|}
\hline M-M' & $\begin{array}{l}\text { Cov. Radius } \\
\text { of } \mathrm{M}[\mathrm{pm}]\end{array}$ & Shortest [ref] & $\begin{array}{l}\text { Distances [pm] } \\
\text { Longest [ref] }\end{array}$ & Average & Distances $>300 \mathrm{pm}$ \\
\hline $\mathrm{Sn}-\mathrm{Ni}$ & 120 & $242.0(1)[151]$ & $253.1(1)[95]$ & 248.5 & $321.1(1)[126]$ \\
\hline Sn-Fe & 120 & $249.9(1)[146]$ & $267.4(1)$ [169] & 257.7 & \\
\hline Sn-Co & 126 & $253.8(5)[150]$ & $268.1(2)[183]$ & 260 & \\
\hline Sn-Ru & 126 & $257.1(1)[172]$ & $269.1(1)[152]$ & 262.8 & \\
\hline $\mathrm{Sn}-\mathrm{Cr}_{\mathrm{r}}$ & 127 & $263.6(3)[106]$ & $272.8(1)$ [159] & 267.5 & $326.7(1)[126]$ \\
\hline Sn-Pt & 128 & $247.8(2)$ [157] & $280.5(2)[180]$ & 264.7 & \\
\hline $\mathrm{Sn}-\mathrm{Os}$ & 128 & 253 [119] & $285.5(2)[177]$ & 270.4 & \\
\hline Sn-Pd & 131 & $251.7(1)[118]$ & $254.0(1)$ [118] & 253.0 & \\
\hline Sn-Rh & 135 & $258.7(2)[153]$ & $260.1(2)[153]$ & 259.2 & \\
\hline Sn-Ir & 137 & $257.5(1)[155]$ & $263.8(1)[156]$ & 260.4 & \\
\hline $\mathrm{Sn}-\mathrm{Nb}$ & 137 & $286.19(5)$ [138] & & & \\
\hline Sn-Mo & 145 & $276.1(1)[106]$ & $281.1(3)[182]$ & 278.6 & $329.2-357.6[161]$ \\
\hline $\mathrm{Sn}-\mathrm{W}$ & 146 & $271.3(2)[162 a]$ & $279.4(2)[162 a]$ & 276.5 & $377.8,385.6[162 b]$ \\
\hline $\mathrm{Sn}-\mathbf{M n}$ & 146 & $244.5(1)[163]$ & $275.8(2)[166]$ & 264.7 & $325.6(1)[126]$ \\
\hline Sn-Re & 159 & $266.6(3)[167]$ & $287.4(3)[167]$ & 276.2 & \\
\hline $\begin{array}{l}\mathrm{Sn}-\mathrm{Sn} \\
\mathrm{Ni}-\mathrm{Ni}\end{array}$ & & $299.1(2)[146]$ & & & $319.1(5)[150]-349.3(1)$ [145] \\
\hline $\begin{array}{l}\mathrm{Ni}-\mathrm{Ni} \\
\mathrm{Fe}-\mathrm{Fe}\end{array}$ & & & & & \\
\hline $\mathrm{Co}-\mathrm{Co}$ & & & & & $392.9(7), 396.7(7)[150]$ \\
\hline Ru-Ru & & $269.5(1)[173]$ & $296.9(1)[172]$ & 286.5 & $345.8(1), 348.1(1)[172]$ \\
\hline $\mathrm{C}_{\mathbf{T}}-\mathrm{C}_{\mathrm{T}}$ & & & & & $306.6(1)[126]$ \\
\hline Pt-Pt & & $260.9(1)[180]$ & $263.9(3)[180]$ & 262.5 & $303.6(2)[157]$ \\
\hline Os-Os & & $274.5(3)[97]$ & $298.9(1)[175]$ & 290.4 & $304.1,305.7[175]$ \\
\hline $\mathrm{Ti}-\mathrm{Ti}$ & & $278.6(3)[136]$ & & & $357.3(1), 363.1(1)[115]$ \\
\hline Ir-Ir & & $272.2(1)[156]$ & $287.8(1)[155]$ & 280 & \\
\hline Mo-Mo & & $272.3(2)[160]$ & $291.1(2)[161]$ & 278.7 & $305.2(2)[161]$ \\
\hline W-W & & $273.0(1)[162 \mathrm{~b}]$ & $274.1(1)[162 b]$ & 273.5 & \\
\hline Mn-Mn & & $261.78(6)[140]$ & & & $306.4(2)[163]-310.45(5)[140]$ \\
\hline Mn-In & & $273.4(3)[106]$ & $274.191)[106]$ & & \\
\hline $\mathrm{Mo}-\mathrm{Hg}_{\mathrm{g}}$ & & $265.9(3)[181]$ & & & \\
\hline Re-Re & & & & & $3013(2)-328.4(3)[141]$ \\
\hline
\end{tabular}

\section{PENTAMERIC HETEROTIN COMPOUNDS}

\subsection{Four Sn plus $M$}

There are almost thirty heteropentameric tin compounds summarised in Table 3. Seven clusters contain four tin plus one other metal, of which the only non-transition example is $\mathrm{Sn}_{4} \mathrm{Al}$ [185]. This molecule approaches $3 \mathrm{~m}$ point symmetry and incorporates a $\mathrm{Sn}_{4} \mathrm{~N}_{3} \mathrm{O}$ cage in a distorted cube arrangement. This can be viewed as two differently sized interpenetrating concentric tetrahedra of $\mathrm{Sn}$ and $\mathrm{N}$ plus $\mathrm{O}$ atoms. The $\mathrm{N}$ atoms carry tert-butyl groups while the $\mathrm{O}$ atom becomes a donor to the AlMez moiety attached to a corner of the cube. The Sn-Sn distances range from 332.0(1) to $337.8(1) \mathrm{pm}$, which excludes any direct metal-metal bond.

Two purple $\mathrm{Sn}_{4} \mathrm{M}$ clusters [186], where $\mathrm{M}$ is $\mathrm{Zr}_{\mathrm{r}}$ or $\mathrm{Hf}$, are isostructural $\left(\mathrm{MSn}_{4} \mathrm{C}_{1}\right)$ with a dodecahedron of Dhd symmetry. This can be viewed as two interpenetrating tetrahedra of carbonyl and triphenylstannyl groups. The $\mathrm{Ph}_{3} \mathrm{Sn}$ units form an elongated tetrahedron (A sites) while the $\mathrm{CO}$ groups form a quite flattened tetrahedron (B sites). The Sn-M distances are 308.6(1) pm for $\mathrm{Zr}$ and 306.3(1) pm for $\mathrm{Hf}$.

A pale yellow compound [187] consists of discrete $\mathrm{NMe}_{4}$ cations and $\mathrm{Sn}_{4} \mathrm{~W}$ cluster anions. The latter contain a central seven coordinate tungsten atom bonded to three terminal $\mathrm{CO}$ groups, two $\mathrm{Pl}_{3} \mathrm{Sn}$ groups and two $\mathrm{Ph}_{2} \mathrm{Sn}$ of the bidentate ligand $(\mathrm{Ph} 2 \mathrm{Sn})_{2} \mathrm{OPr}$. The $\mathrm{Sn}-\mathrm{W}$ bond distances range from $276.0(2)$ to $281.1(2) \mathrm{pm}$ (average $278 \mathrm{pm}$ ). The seven donor atoms are in an approximate 4:3 piano stool arrangement about the central tungsten ( $\mathrm{WSn}_{4} \mathrm{C}_{3}$ ). Each $\mathrm{Sn}$ atom has a quasi-tetrahedral environment with either $\mathrm{SnC}_{2} \mathrm{OW}$ or $\mathrm{SnC}_{3} \mathrm{~W}$ chromophores.

There are two $\mathrm{Sn}_{4} \mathrm{Ni}$ clusters, dark red [188]and green-black [189]. In the former a central Ni(0) atom is almost tetrahedrally surrounded by $\mathrm{Sn}\left(\mathrm{NBu}^{\prime}\right)_{2} \mathrm{SiMe}_{3}$ moieties. The $\mathrm{SnN}_{2} \mathrm{Si}$ groups are skewed with respect to each other and degrade the three-fold symmetry of a regular tetrahedron $\left(\mathrm{NiSn}_{4}\right)$ giving rise to severe distortions. However, the $\mathrm{Sn}-\mathrm{Ni}$ bond distances are remarkably constant at a mean value of 239.4(1) pm (maximum deviation $0.6 \mathrm{pm}$ ), which is about $17 \mathrm{pm}$ smaller than the sum of the covalent radii of $\mathrm{Sn}$ and $\mathrm{Ni}$. This implies considerable double bond character which may be due to $\mathrm{d} \pi$-p $\pi$ back donation in the $\mathrm{Sn}-\mathrm{Ni}$ bond [188]. The second derivative has the central $\mathrm{Ni}$ atom in a cavity of the (pz)S macrocyclic ligand, coodinated by four $\mathrm{N}$ 
Heterometallic Tin Compounds: Classification and Analysis of Crystallographic and Structural Data : Part II.

atoms in a square planar arrangement. The four $\mathrm{SnBu}{ }^{\dagger}$ moieties are located around the macrocycle in a star fashion. Each $\mathrm{Sn}$ atom is coordinated to two thiolates of different pyrrole rings and also to the meso nitrogen of a porphyrazine. A nearly square-pyramidal geometry about each tin is completed by two tert-butyl groups, one being at the apex. Two independent tin atoms are adjacent and lie $73 \mathrm{pm}$ and $54 \mathrm{pm}$ above the plane of the planar porphyrasineoctathiolate ligand. The other two tin atoms are related by inversion and lie below this plane, resulting in a quasi-chair conformation.

A pale yellow $\mathrm{Sn} 4 \mathrm{Pt}$ cluster [190] has a hexacoodinate $\mathrm{Pt}(\mathrm{IV})$ species with four $\mathrm{Sn}-\mathrm{Pt}$ bonds. Two of these are to $\mathrm{Sn}\left(\mathrm{p}-\mathrm{MeC}_{6} \mathrm{H}_{4}\right)_{2}(\mu-\mathrm{OMe})$ moities, one is to $\mathrm{Sn}\left(\mathrm{p}-\mathrm{MeC}_{6} \mathrm{H}_{4}\right)_{3}$ and one to a neutral $\mathrm{Sn} "\left(\mathrm{P}-\mathrm{MeC}_{6} \mathrm{H}_{4}\right)$ unit. The Sn-Pt bond distances range from 258.591) to $264.5(1) \mathrm{pm}$ (average $261.3 \mathrm{pm}$ ). The mean Sn-Pt-Sn angles are $80.6^{\circ}$ and $95.6^{\circ}$.

\subsection{Three Sn plus Two $M$}

A colourless $\mathrm{Sn}_{3} \mathrm{Li} 2$ cluster [191] shown inFigure 8 is a trigonal prismatic unit built up from three stacked triangular units on top of one another. The central triangle is $\mathrm{Sn}_{3} \mathrm{O}$ in which the $\mathrm{O}$ atom bridges all three $\mathrm{Sn}$ atoms. The local symmetry about each $\mathrm{Sn}$ atom is distorted trigonal bipyramidal, with $\mathrm{O}$ atoms of the cyclometallated alkoxide groups being in mutually trans axial positions. These are also bonded to a lithium atom in the top and bottom $\mathrm{LiO}_{3}$ triangles of the cluster core.

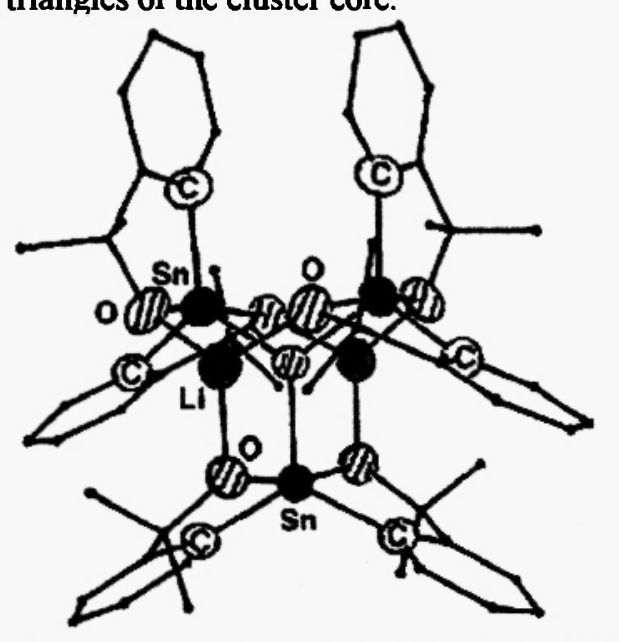

Fig.8. Structure of $\left[\left\{\mathrm{Sn}(\mathrm{phpr})_{2}\right\}_{3} \mathrm{Li}_{2} \mathrm{O}\right][191]$

The structure of a deep orange $\mathrm{Sn}_{3} \mathrm{Pt}_{2}$ cluster [49] consists of a trigonal bipyramid with the $\mathrm{Pt}$ atoms separated from one another by $259.3(1) \mathrm{pm}$ and with bond angles of $18.9^{\circ}$ (P-Pt-Sn) and $98.6^{\circ}$ (Sn-Pt-Sn). The two apical coordination positions at Pt are occupied by $\mathrm{PPh} 3$ molecules, while the three equatorially bridging ligands are symmetrically disposed with a Pt-Sn distance of $268.0(1)$ pm and Pt-Sn-Pt angle of 57.87(3)'. This geometry represents face sharing of two distorted PPtSnz fragments. Two chelated (acac) groups complete an octahedral coordination about each $\mathrm{Sn}(\mathrm{II})$ atom ( $\mathrm{SnO}_{\mathrm{P}} \mathrm{P}$ ).

\subsection{Two Sn plus Three $M$}

An orange brown complex [36] contains a cobalt cation $\left.\left[\mathrm{Co}\{\mathrm{pz})_{3} \mathrm{BH}\right\}_{2}\right]^{+}$with a $\mathrm{Sn}_{2} \mathrm{Co}_{3}$ cluster anion $\left[\left\{(\mathrm{CO})_{3} \mathrm{CoSn}(\mathrm{Cl})_{2}\right\}_{2} \mathrm{Co}(\mathrm{CO})_{3}\right]$. The anion has a planar central $\mathrm{Co}(\mathrm{CO})_{3}$ unit with two $\mathrm{SnCl}_{2}\left\{\mathrm{Co}(\mathrm{CO})_{4}\right\}$ units bonded in a trans arrangement $\left[\mathrm{Co}(2)-\mathrm{Sn}(1)-\mathrm{Co}(1)-\mathrm{Sn}\left(1^{1}\right)-\mathrm{Co}(2)\right]$. The $\mathrm{Sn}(1)-\mathrm{Co}(1)$ bond distance of 246.891) pm is shorter than that of $\mathrm{Sn}(1)-\mathrm{Co}(2)$ at $256.2(1) \mathrm{pm}$. The $\mathrm{Sn}(1)-\mathrm{Co}(1)-\mathrm{Sn}\left(1^{\prime}\right)$ angle is almost linear $(176.5(1)$. The other two $\mathrm{Co}(1)-\mathrm{Sn}(1)-\mathrm{Co}(2)$ angles are bent at $127.5(1)$.

There are two isostructural $\mathrm{Sn}_{2} \mathrm{M}_{3}$ clusters [192], one with ruthenium and one with osmium. The structures show a planar array of five metal atoms, with $\mathrm{Sn}$ atoms bridging two edges of the $\mathrm{M}_{3}$ triangle, the third edge being bridged equatorially by a carbonyl group. The mean $\mathrm{Sn}-\mathrm{Ru}$ and $\mathrm{Sn}-\mathrm{Os}$ distances are 273.592) and $273.7(0) \mathrm{pm}$, respectively. The Ru-Ru distance of $293.1(2) \mathrm{pm}$ is smaller than the Os-Os distance of 294.7(0) pm as expected.

The yellow $\mathrm{Sn}_{2} \mathrm{Pd} d_{3}$ cluster [193] has a distorted propeller shaped $\mathrm{Pd}_{3} \mathrm{Sn}_{2}$ frame composed of three triangles, $\mathrm{Pd} 3$ and two $\mathrm{Pd} 2 \mathrm{Sn}$ with a mutual Pd-Pd edge. There are two short Pd-Pd bonds (270.8(1) and 272.0(1) pm) with the Pd atoms linked by slightly assymetrical $\mathrm{CO}$ bridges. The Pd-Sn bond lengths are 267.9 (1) to $272.1(1) \mathrm{pm}$ with a mean value of $269.7(1) \mathrm{pm}$. The $\mathrm{Sn}$ (II) atoms are pseudo-octahedrally 
coordinated $\left(\mathrm{SnO}_{4} \mathrm{Pd} 2\right)$.

An amber $\mathrm{Sn}_{2} \mathrm{O} \mathrm{s}_{3}$ cluster [194] contains an osmium triangle with three different Os-Os distances, 304.8(2), 285.4(2) and 290.9(2) pm. The Os-Os-Os angle deviates from 60(+/-3.8) ${ }^{\circ}$. Each of the two $\mathrm{Sn}\{\mathrm{CH} \text { (SiMes }\}_{2}$ moieties is bound to two Os atoms, sharing one, with distances of 287.3(3) and 264.9(3) pm and 286.6(3) and 265.1(2) pm. The structure of a colourless $\mathrm{Sn}_{2} \mathrm{Oss}_{3}$ cluster consists of an Os3 triangle, two edges of which are bridged by hydrogen ligands. The bridged edges are longer (302.1(3) and 307.0(2) pm) than the unbridged edge (289.6(3) pm. Two SnMes moieties are bonded to one Os atom with distances of 272.6(5) and 269.6(4) pm.

There are two coloured $\mathrm{Sn}_{2} \mathrm{Pt} 3$ clusters [196,197]. The first is a red derivative [196] consisting of a triangle of $\mathrm{Pt}$ atoms each coordinated to a $\eta^{3}$-cycloocta-1,5-diene ring and capped above and below by $\mathrm{SnCl}_{3}$ moieties. The average distances are 258(1) pm for three Pt-Pt distances, 280(1) pm for six Pt-Sn distances and 239(2) pm for six Sn-Cl distances. The mean bond angles are $60.0^{\circ}$ for Pt-Pt-Pt, $54.9^{\circ}$ for Pt-Sn-Pt and $62.5^{\circ}$ for Pt-Pt-Sn. The orange cluster [197] also contains a Pt triangle (Pt-Pt distances 260.9(2), 262.2(1) and 263.9(1) pm) with the edge of the triangle bridged by a $\mu$-dppm ligand and each face bridged by a $\mu_{3}-\mathrm{SnF}_{3}$ moiety. Thus the Pt3Snz cluster adopts a slightly distorted trigonal bipyramidal geometry, with two apical $\mu_{3-}$ $\mathrm{SnF}_{3}$ moieties in an eclipsed orientation with respect to each other. The $\mathrm{Sn}$ (II) atom has an approximately octahedral environment $\left(\mathrm{SnF}_{3} \mathrm{Pt}\right.$ ). The $\mathrm{Sn}$-Pt bond distances range from 269.9(2) to 283.0(2) pm (average 278 pm).

The structure of a colourless $\mathrm{SnCu}_{4}$ cluster [198] is shown in Figure 9. A C $3 v$ symmetry hyphohexagonal metal core is linked by a combination of $\mathrm{N}, \mathrm{S}$ and $\mathrm{N}, \mathrm{i}-\mathrm{S}$-chelating (mit) ligands to form a cage-like molecule. The Sn(II) atom is bonded to the N-centres of three (mit) ligands. Three symmetry related ligands form bridges between the $\mathrm{Sn}$ atom and each of the equatorial $\mathrm{Cu}(\mathrm{I})$ ligands of the core $(\mathrm{S}-\mathrm{Cu}=226.6(2) \mathrm{pm})$ with the remaining mit ligands $\mathrm{N}$, i-S-chelating the three available $\mathrm{Cu}_{3}$ faces of the $\mathrm{Cu}_{4}$ pyramid. The $\mathrm{Cu}-\mathrm{Sn}$ distances are 272.7(2) and 290.2(1) pm. The Cu-Cu distances range from 267.1(2) to 313.2(2) pm.

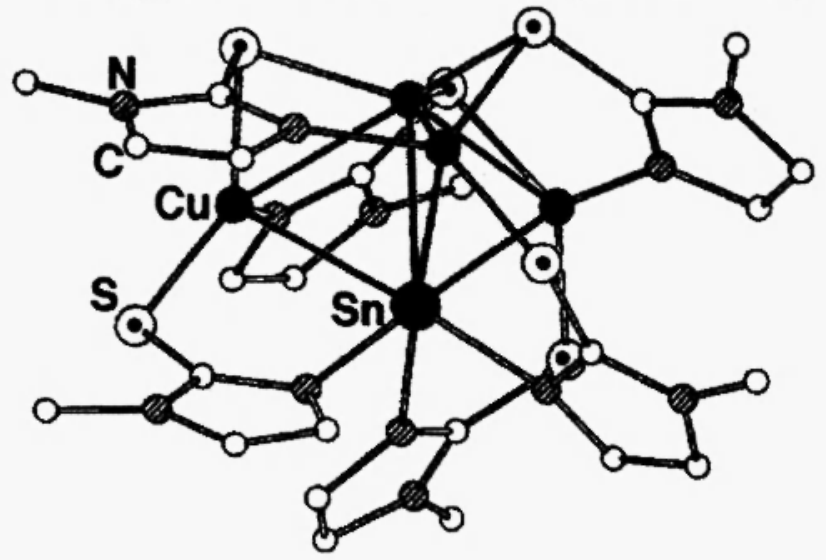

Fig9. Structure of [Sn(mit)6Cu4] [198]

Crystals of a SnRe4 cluster [199] reveals two $\operatorname{Re}_{2} \mathrm{P}_{4}$ units forming an elongated tetrahedron about the central tin atom. The resulting metallo-spiropentane displays a curious distortion about the tin, as demonstrated by the Sn-Re distances ranging from $262.7(2)$ to $279.3(2) \mathrm{pm}$.

There are two $\mathrm{SnFe}_{4}$ clusters $[86,200]$. The former [86] contains two triangles of the metal atoms which share the tin atom. There are two pairs of Sn-Fe bond distances, one of 254.6(4) pm and the other of 255.7(4) $\pm 0.2 \mathrm{pm}$. The Fe-Fe edges are equal with a distance of $289.0(5) \mathrm{pm}$. Each iron atom is also bonded to four terminal carbonyl groups. The latter cluster [200] exhibits a distorted tetrahedral configuration around the central $\mathrm{Sn}(\mathrm{IV})$ atom, with two $\mathrm{Fe}(\mathrm{CO})_{4}$ groups $(\mathrm{Sn}-\mathrm{Fe}=258.8(3)$ and $258.9(3) \mathrm{pm})$ and one $\left[\mathrm{Fe} 2(\mathrm{CO})_{8}\right]^{*}$ unit $(\mathrm{Sn}-\mathrm{Fe}=$ 272.5(3) and 273.4(3) pm). Two iron atoms in the latter unit are bridged by two carbonyl groups with $\mathrm{Fe}-\mathrm{Fe}$ bond distance of $260.5(3) \mathrm{pm}$.

Red SnCo4(CO) $)_{16}$ was studied by two groups $[201,202]$ and their data correspond well. The tetrahedral Sn atom sits on a site of 23 symmetry and is surrounded by four trigonal bipyramidal $\mathrm{Co}(\mathrm{CO})_{4}$ groups with equal bond distances, 266.9(1) pm $\left\lceil 2011\right.$ and 267.03(14) pm [202]. The structure of $\mathrm{Ph}_{2} \mathrm{Sn}\{(\mu$ $\left.\left.\eta^{2} \mathrm{C}_{2} \mathrm{H}\right) \mathrm{CO}_{2}(\mathrm{CO})_{8}\right\}_{2}$ [203] is shown irFigure 10. A tetrahedral arrangement about the central tin atom is built up from two $\left(\mu-\eta-\mathrm{C}_{2} \mathrm{H}\right) \mathrm{CO}_{2}(\mathrm{CO})_{8}$ moieties with symmetrically $\mathrm{Sn}-\mathrm{C}$ bond distances (212.2(7) and 212.3(7) pm). The Co-Co bond distances in the respective two moieties are 247.8(2) and 248.7(2) pm. 


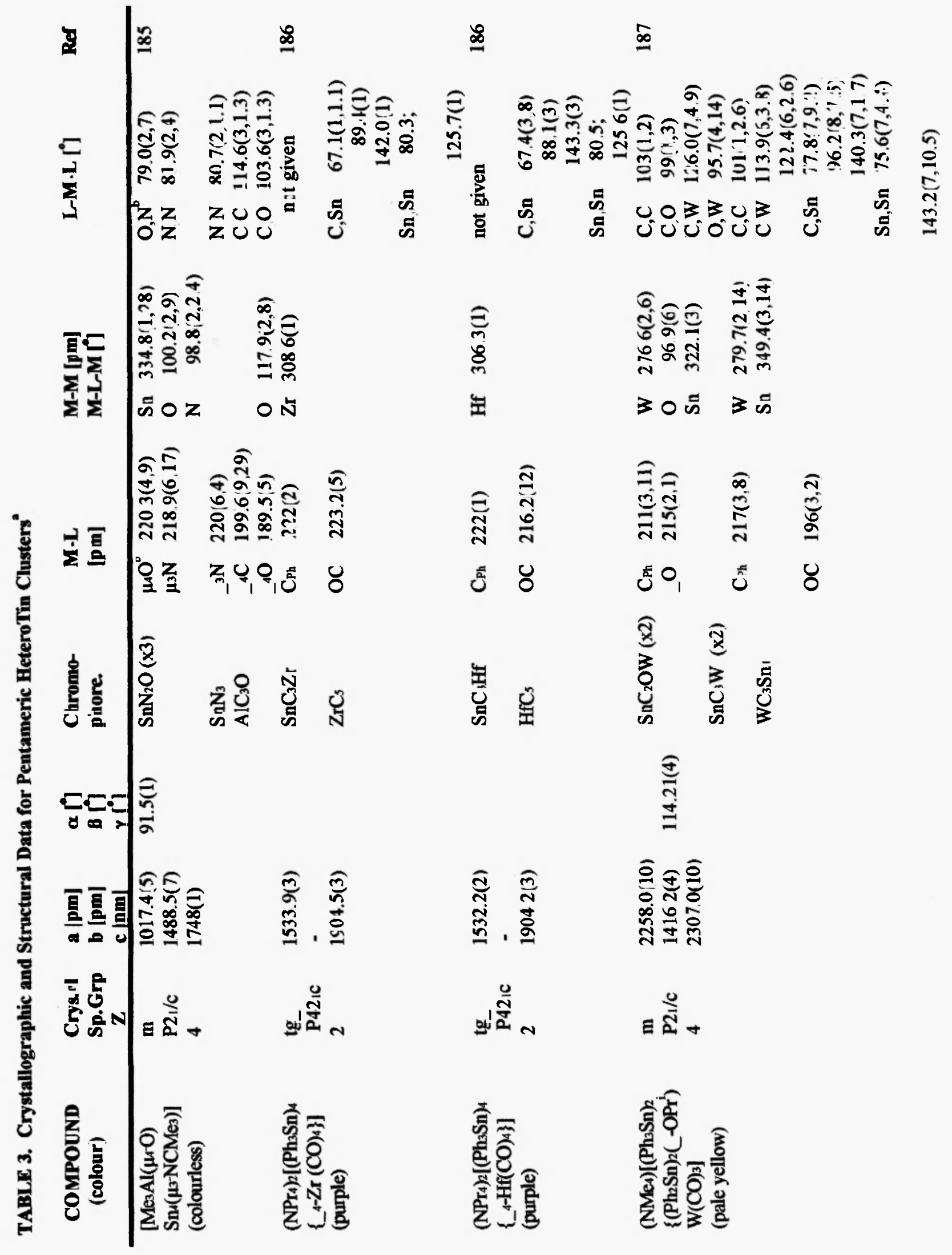


$\stackrel{\infty}{\infty}$

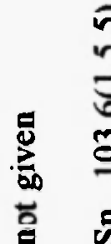

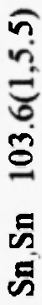

Ð



$\bar{\Xi}$

q i

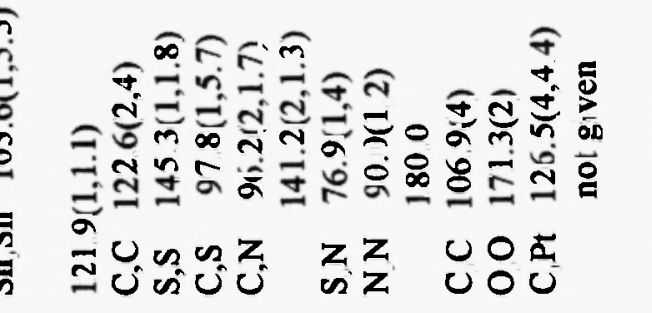

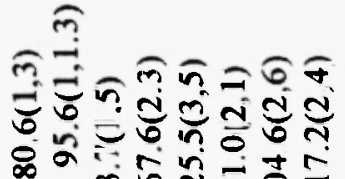

車气志

हี 0 บับ

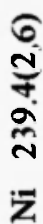

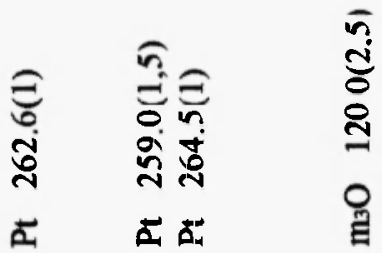

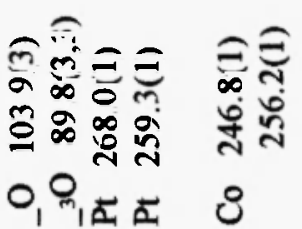

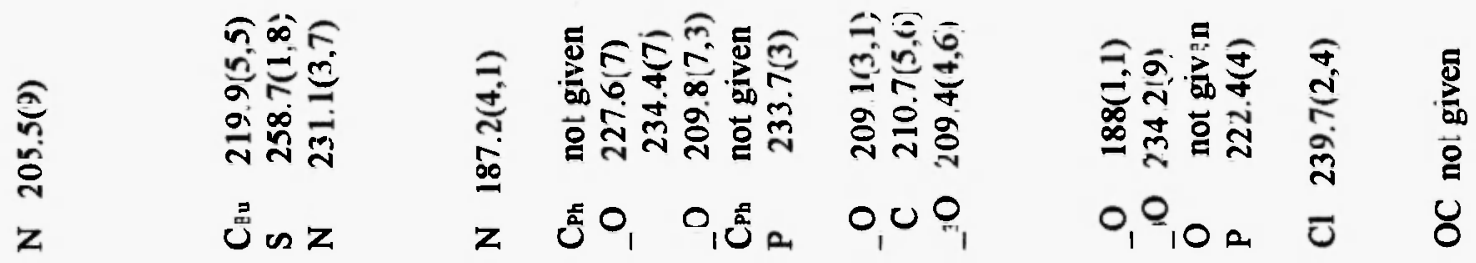

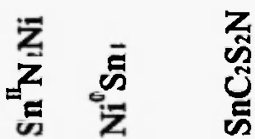

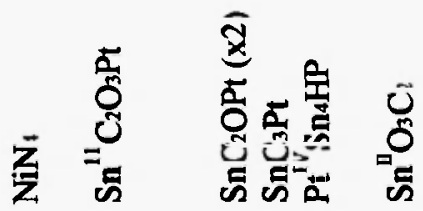

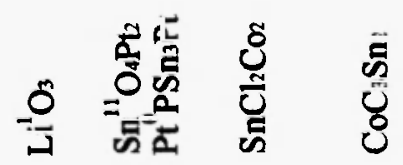

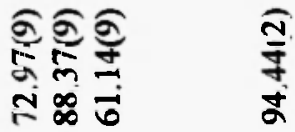

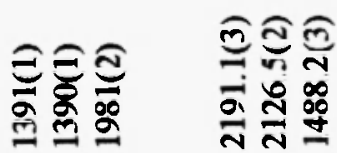

ที丶

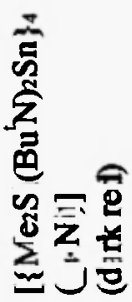

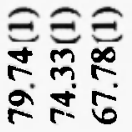

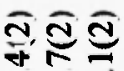

赵家

$\cong \stackrel{\mathrm{N}}{ }$

ที⿰丨丨

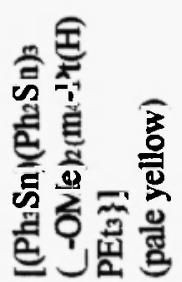

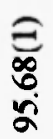

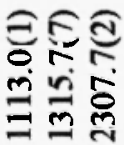

ถ

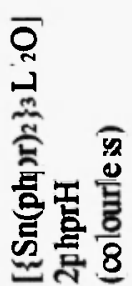

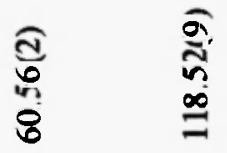

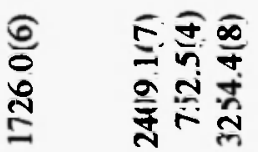


$\approx$

$\stackrel{2}{2}$

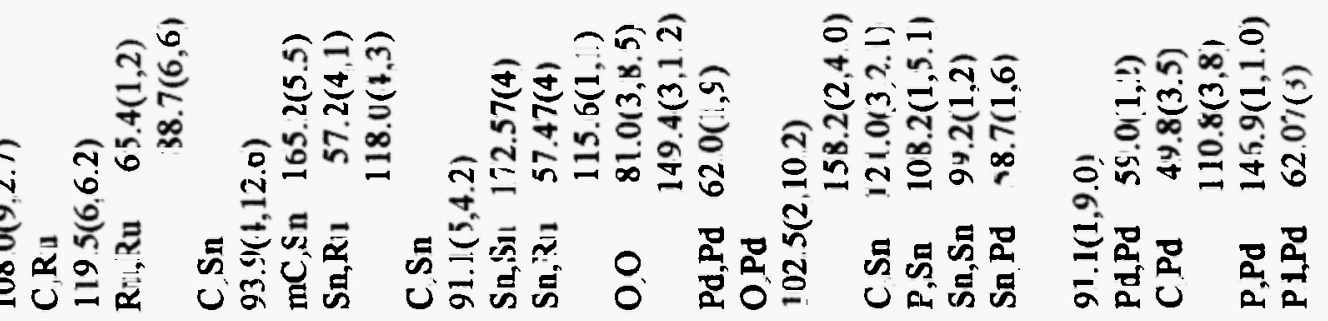

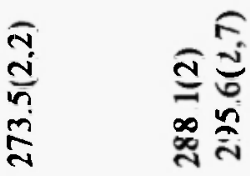

\begin{tabular}{|c|}
\hline 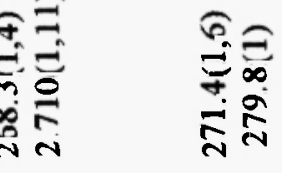 \\
\hline \\
\hline
\end{tabular}

a $\vec{x}$

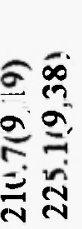

$\circ$

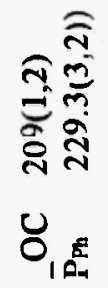

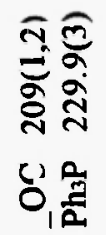

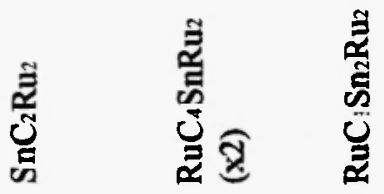

ถี

䒾

$\frac{\pi}{0}$

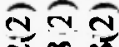

닝

气 :

ڤ్

金勇吉

它离宫

$\widehat{\widehat{ }} \cong$

$\infty$ it

$\underline{\infty}$

ที่ั<smiles>[13CH3][13CH3]</smiles>

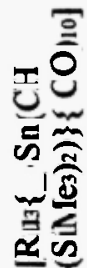

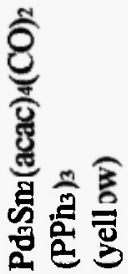


I

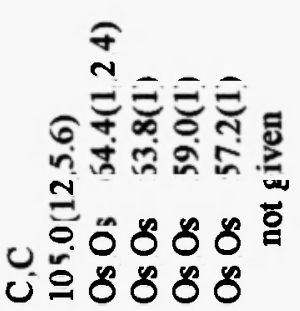

$\cong$

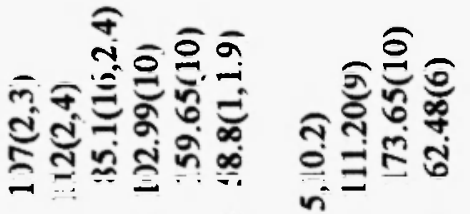

$\stackrel{5}{\Xi}$

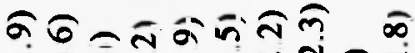

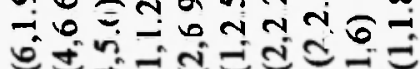

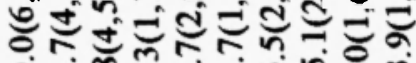

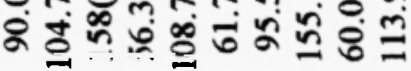
용 की

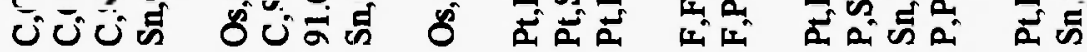

$=F^{20}$

çำ

i.

议

888

\section{$\underline{6}$}

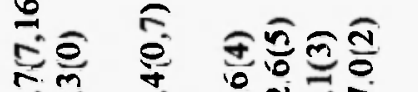

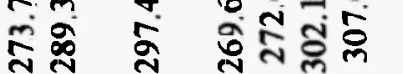

88 \& $88 \%$

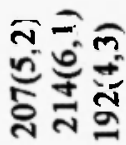

ปี

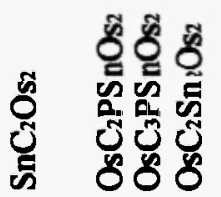

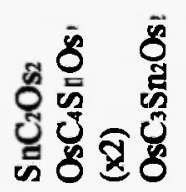

$\varnothing_{0}$ 密

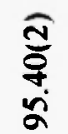

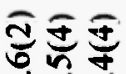

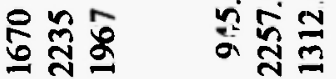

ะ 气ัญ

ะ ⿱ㅗำ

\begin{tabular}{c}
$n$ \\
\multirow{2}{*}{} \\
0
\end{tabular}

8

突

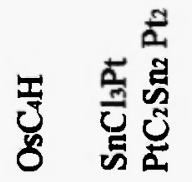

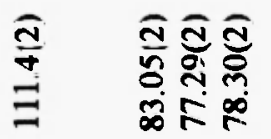

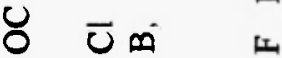

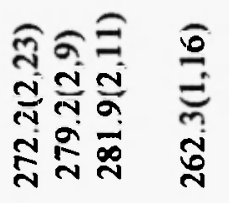

医

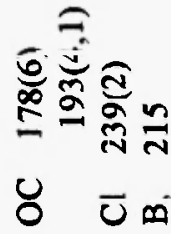

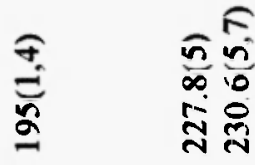

L $D$

a
$\Rightarrow \ln \alpha$

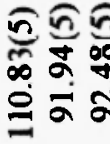

오ำำำ

을

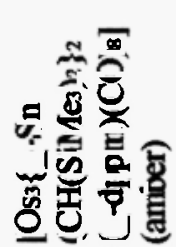

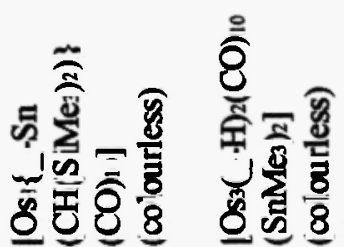

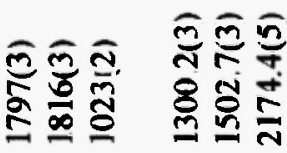

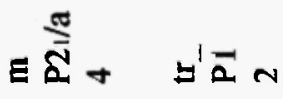

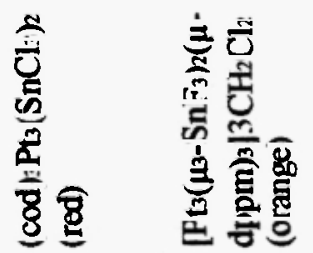


$\stackrel{\circ}{9}$

๖ \& 유

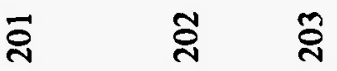

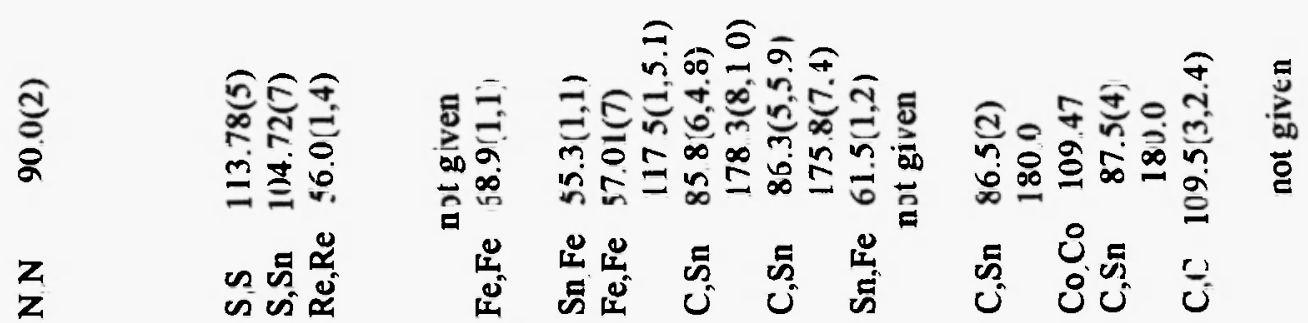

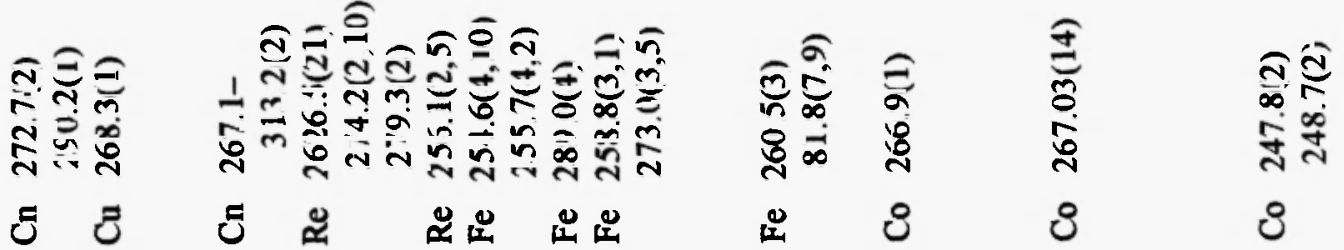

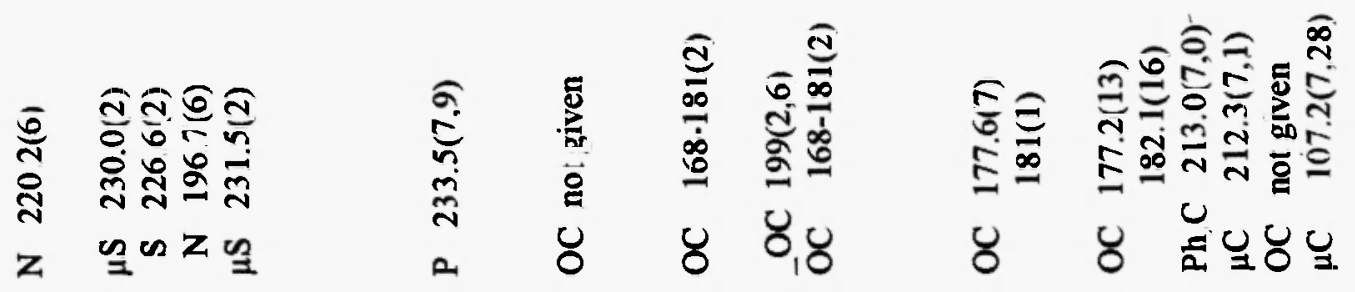

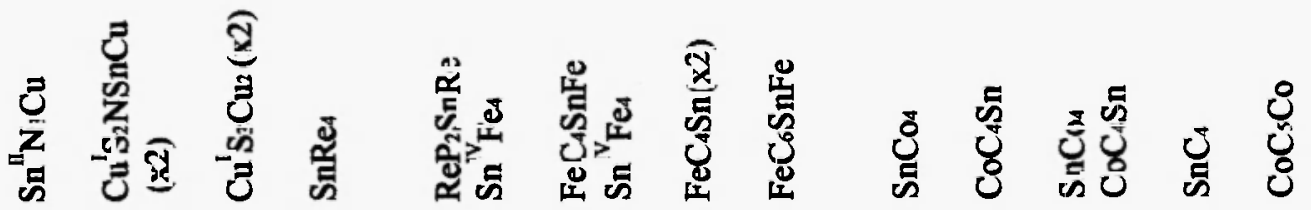<smiles>[CH]C=[CH]</smiles>

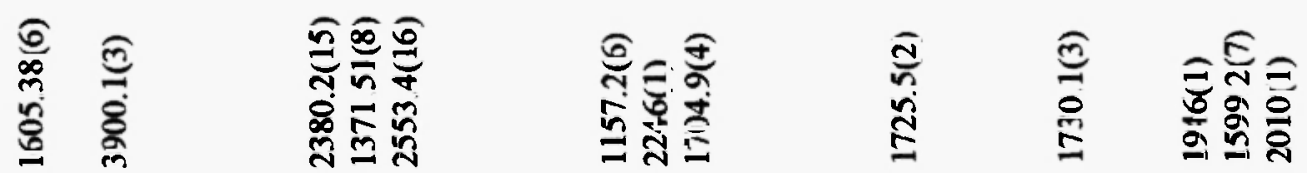

$\operatorname{Eี}^{\prime} \tilde{\varkappa}$

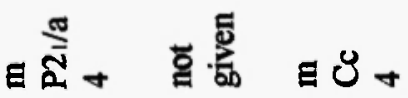

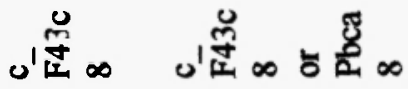
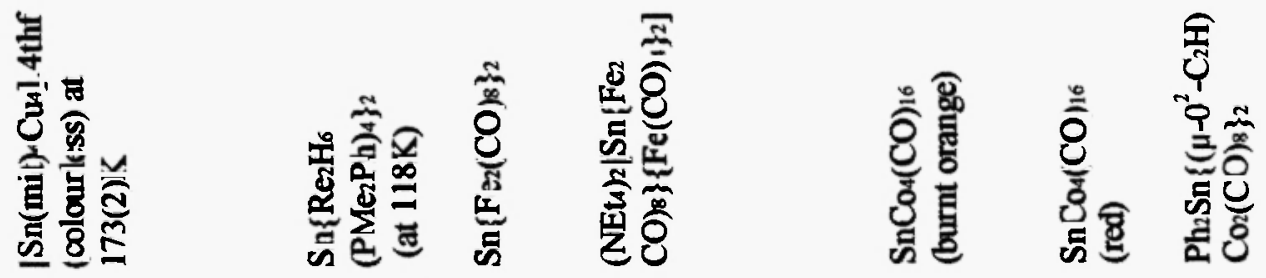


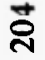

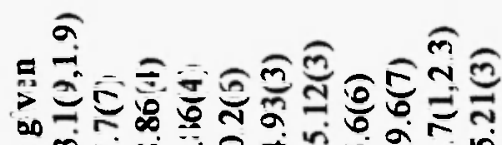

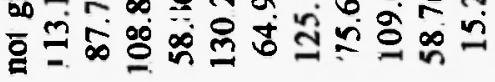

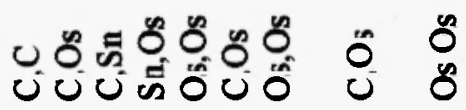

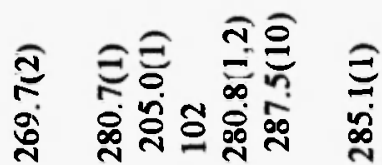

ำ

$8 \%=8$

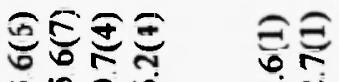

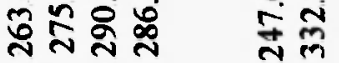

$\delta 38 \delta$ 뭉

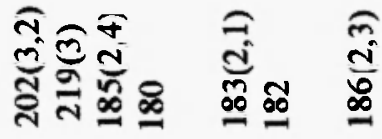

文

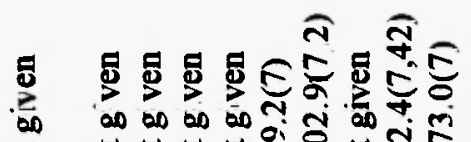

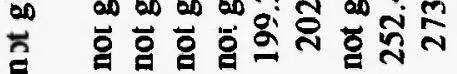

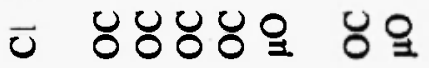

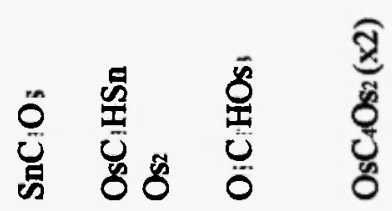

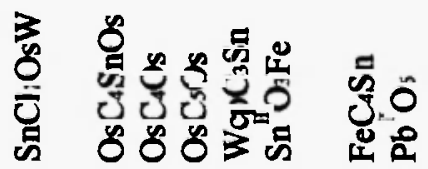

고ㅇㅝㅠ

$\dot{\infty} \vec{\infty}$

छสำส

ูํํำ

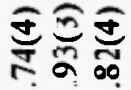

으용

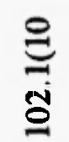

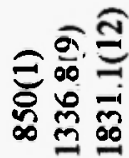

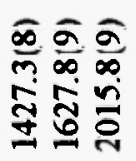

ำ

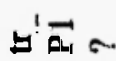

ต $\stackrel{0}{0}+$

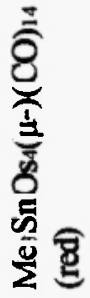




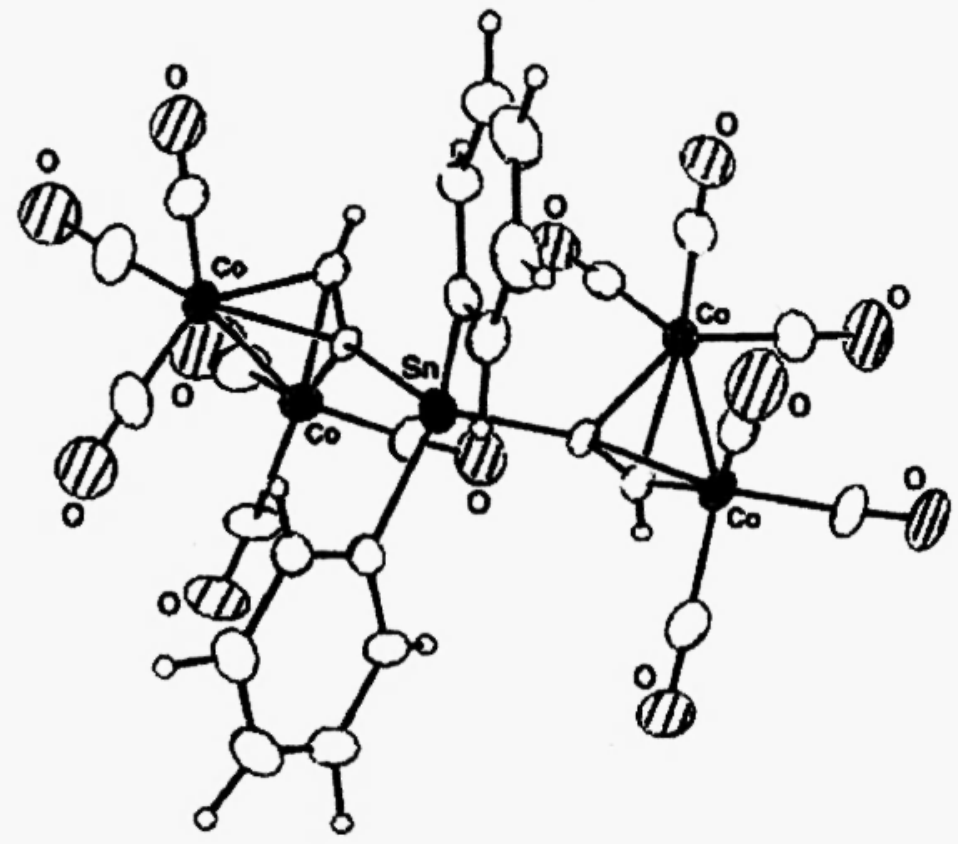

Fig.10. Structure of $\left.\mathrm{Ph}_{2} \mathrm{Sn}\left\{\left(\mu-\eta^{2}-\mathrm{C}_{2} \mathrm{H}\right) \mathrm{Co}_{2}(\mathrm{CO})\right\}_{2}\right\}_{2}$ [203]

In a red SnOsA cluster [204] the osmium atoms adopt a planar butterfly arrangement with Os-Os distances of $280.7(1), 287.5(1), 281.0(1), 305.0(1)$ and $285.1(1) \mathrm{pm}$. The Sn atom lies approximately in the Os4 plane attached to one of the "wingtip" Os atoms (Sn-Os $=269.7(2) \mathrm{pm}$, and cis to the neighbouring Os atom).Three methyl groups complete the distorted tetrahedral configuration about the tin atom.

Table 3A. Summary of Sn-M and M-M Distances in Pentameric heterotin Clusters

\begin{tabular}{|c|c|c|c|c|c|}
\hline$M-M^{\prime}$ & $\begin{array}{l}\text { Cov. Radius } \\
\text { of } M^{\prime}[\mathrm{pm}]\end{array}$ & Shortest [ref] & $\begin{array}{l}\text { Distances [pm] } \\
\text { Longest [ref] }\end{array}$ & Average & Distances $>300 \mathrm{pm}$ \\
\hline Sn-Al & 118 & & & & $332.0-337.891)[185]$ \\
\hline $\mathrm{Sn}-\mathrm{Zr}_{\mathbf{r}}$ & 148 & & & & $308.6(1)[186]$ \\
\hline Sn-Hf & 149 & & & & $306.1(1)[186]$ \\
\hline $\mathrm{Sn}-\mathrm{Pb}$ & 131 & & & & $332.7(1)[206]$ \\
\hline Sn-Sn & & & & & $322.1(3)-350.7(3)[187]$ \\
\hline $\mathrm{Sn}-\mathrm{Ni}$ & 120 & $239.3(2)[188]$ & $239.9(2)[188]$ & 239.4 & \\
\hline $\mathrm{Sn}-\mathrm{Fe}$ & 120 & $247.6(1)[206]$ & $273.4(3)[200]$ & 259.0 & \\
\hline Sn-Co & 126 & $246.8(1)[36]$ & $267.0(1)[202]$ & 259.2 & \\
\hline Sn-Ru & 126 & $273.3(2)[192]$ & $273.7(2)[192]$ & 273.5 & \\
\hline Sn-Pt & 128 & $258.5(1)[190]$ & $283.0(2)$ [197] & 272.0 & \\
\hline Sn-Os & 128 & $263.6(6)[205]$ & $286.6(3)[194]$ & 272.8 & \\
\hline Sn-Pd & 131 & $267.9(2)[193]$ & $272.1(2)[193]$ & 269.6 & \\
\hline $\mathrm{Sn}-\mathrm{Cu}$ & 138 & $272.7(1)[198]$ & $290.2(1)[198]$ & 281.4 & \\
\hline $\mathrm{Sn}-\mathrm{W}$ & 146 & $275.6(7)$ [205] & & & \\
\hline Sn-Rè & 159 & 262.6(1) [199] & 279.3(2) [199] & 272.6 & \\
\hline $\mathrm{Fe}-\mathrm{Fe}$ & & $260.5(3)[200]$ & $289.0(4)[200]$ & 274.7 & \\
\hline $\mathrm{Co}-\mathrm{Co}$ & & $247.8(2)[203]$ & $248.7(2)[203]$ & 248.2 & \\
\hline Ru-Ru & & $288.1(2)[192]$ & $296.3(2)[192]$ & 292.5 & \\
\hline Pt-Pt & & $258(1)[196]$ & $259.3(1)$ [49] & 277 & \\
\hline Os-Os & & $270.7(4)$ [205] & $298.1(2)$ [192] & 286.8 & $302.1(3)-307.0(3)[195]$ \\
\hline Pd-Pd & & $270.8(1)[193]$ & $279.8(1)$ [193] & 274.2 & \\
\hline $\mathrm{Cu}=\mathrm{Cu}$ & & $260.3(1)$ [198] & $268.3(1)$ [198] & 264.3 & $313.2(2)[198]$ \\
\hline $\mathrm{Re}-\mathrm{Re}$ & & $255.5(2)$ & $256.6(2)[1991$ & 256.0 & \\
\hline
\end{tabular}

A $\mathrm{SnO}_{3} \mathrm{~W}$ cluster [205] has a linear tri-osmium backbone $\left(\mathrm{Os}-\mathrm{Os}-\mathrm{Os}=175.9(2)^{\circ}\right.$. The $\mathrm{SnCl}_{2}\left(\mathrm{cpW}\left(\mathrm{CO}_{3}\right)_{3}\right.$ occupies an axial position on one terminal osmium, also making a linear $\mathrm{O}_{3} \mathrm{Sn}$ chain (Os-Os$\mathrm{Sn}=175.8(2)^{\circ}$, and $\left.\mathrm{Os}-\mathrm{Sn}=263.6(6) \mathrm{pm}\right)$. The tungsten is bonded to tin $(\mathrm{Sn}-\mathrm{W}=275.6(7) \mathrm{pm})$ to give a 
hetero-pentametallic chain with no bridging ligands. The Os-Sn-W angle is $132.8(2)^{\circ}$.

A gold yellow $\mathrm{Sn}_{2} \mathrm{Fe} 2 \mathrm{~Pb}$ cluster [206] has a crystallographic twofold axis passing through the $\mathrm{Pb}$ (II) atom. The metal atoms are coupled one-dimensionally, $(\mathrm{CO})_{4} \mathrm{Fe}-\mathrm{Sn}(\mu-\mathrm{OBu})_{3} \mathrm{~Pb}(\mu-\mathrm{OBu})_{3} \mathrm{Sn}-\mathrm{Fe}(\mathrm{CO})_{4}$, with equal $\mathrm{Sn}-\mathrm{Fe}$ bond distances of $247.6(1) \mathrm{pm}$. Each $\mathrm{Sn}(\mathrm{II})$ atom has a quasi-tetrahedral environment $\left(\mathrm{SnO}_{3} \mathrm{Fe}\right)$. The central $\mathrm{Pb}(\mathrm{ll})$ atom has a quasi-octahedral environment $\left(\mathrm{PbO}_{6}\right)$.

The data in Table 3 shows six categories of the pentameric derivatives, $\mathrm{Sn}_{4} \mathrm{M}$ (7 examples), $\mathrm{Sn}_{3} \mathrm{M}_{2}(2$ examples), $\mathrm{Snz}_{3}$ (8 examples), $\mathrm{SnM}_{4}\left(8\right.$ examples), $\mathrm{SnM}_{3} \mathrm{M}^{\prime}$ (1 example) and $\mathrm{Sn}_{2} \mathrm{M}_{2} \mathrm{M}^{\prime}$ (1 example). The most common environment about tin is distorted tetrahedral. A summary of the $\mathrm{Sn}-\mathrm{M}$ and $\mathrm{M}-\mathrm{M}$ distances in the pentameric derivatives is found in Table 3A. The shortest bond distance is $\mathrm{Sn}-\mathrm{Ni}$ at 239.3(2) pm [188]. Notably, the mean Sn-M bond distances in the pentameric derivatives are generally longer than those in the tri- and tetrameric derivatives, except for $\mathrm{Sn}-\mathrm{Ni}$ and $\mathrm{Sn}-\mathrm{Re} .5$.

\section{HEXAMERIC HETEROTIN COMPOUNDS}

Crystallographic and structural data for over forty hexameric heterotin clusters are summarised in Table 4. There are six subgroups based on the metal content ratios: $\mathrm{SnsM}$ (5 examples); $\mathrm{Sn}_{4} \mathrm{M}_{2}$ (10 examples); $\mathrm{Sn}_{3} \mathrm{M}_{3}$ (7 examples); $\mathrm{Sin}_{2} \mathrm{M}_{3} \mathrm{M}^{\prime}$ (1 example) and $\mathrm{Sn}_{2} \mathrm{M}_{2} \mathrm{M}_{2}$ (2-examples).

\subsection{Five Sn plus $M$}

The bright orange $\mathrm{SnsRu}$ cluster [207] has a central Ru(II) atom coordinated to five $\mathrm{SnCl}_{3}$ moieties and a CI atom. The Sn-Ru bond distance ranges from $255.3(2)$ to $258.6(1) \mathrm{pm}$ (average $257.4 \mathrm{pm}$ ). Each $\mathrm{Sn}$ atom is quasi-tetrahedral ( $\mathrm{SnCl}_{3} \mathrm{Ru}$ ) while the Ru atom is six coordinate (RuCISns). In a dark red SnsRh cluster [42] the central $\mathrm{Rh}(\mathrm{I})$ atom is bonded by only the five $\mathrm{Sn}\left\{(\mathrm{NBu})_{2} \mathrm{SiMe}_{2}\right\}$ moieties. Two $\mathrm{Sn}(\mathrm{II})$ atoms are nonsymmetrically bridged by a chlorine atom $(\mathrm{Sn}-\mathrm{Cl}=258.5(3)$ and $273.8(3) \mathrm{pm}$, respectively). The mean $\mathrm{Sn}-\mathrm{Rh}$ distance for these two $\mathrm{Sn}$ atoms is about $3 \mathrm{pm}$ longer than the mean $\mathrm{Sn}$-Rh distance for the remaining three $\mathrm{Sn}$ (II) atoms (254.1 pm). The Rh(I) atom has almost ideal trigonal-bipyramidal geometry (RhSns). There are two pairs of $\mathrm{Sn}$ (II) atoms, one in a Y-shaped environment $\left(\mathrm{SnN}_{2} \mathrm{Rh}\right)$ and the other a distorted tetrahedron $\left(\mathrm{SnN}_{2} \mathrm{ClRh}\right.$ ). Two crystallographically independent molecules are found in a yellow-orange SnOss cluster with identical configurations differing by degree of distortion. Each Os atom is octahedrally coordinated (OsClSns). The $\mathrm{Sn}$-Os bond distance ranges from 253.1 to $260.0 \mathrm{pm}$. Each Sn(II) atom has a quasi-tetrahedral environment ( $\mathrm{SnCl} 3 \mathrm{Os})$. Another yellow SnsOs cluster [209] is isostructural with the previous structure [208] and also contains two crystallographically independent molecules. In the red SnsPt cluster the $\left[\mathrm{Pt}\left(\mathrm{SnCl}_{5}\right)_{s}\right]^{-3}$ anion is a regular trigonal bipyramid with average axial $\mathrm{Sn}-\mathrm{Pt}$ bond length of $255.30(7) \mathrm{pm}$, a little shorter than the equatorial Sn-Pt value of $257.22(10) \mathrm{pm}$. The $\mathrm{Sn}$ (II) atoms are pseudo-tetrahedrally coordinated ( $\mathrm{SnCl} 3 \mathrm{Pt})$. This subgroup of the hexameric heterotin clusters contains tin only in the oxidation state of two, $\mathrm{Sn}$ (II).

\subsection{Four Sn plus Two $M$}

The $\mathrm{Sn}_{4} \mathrm{M}_{2}$ clusters have ten examples, the first of which is a non-transition metal derivative shown in Figure 11 [211]. This consists of a central $\mathrm{Li}_{2} \mathrm{Br}$ four membered ring with a crystallographic inversion centre, with each $\mathrm{Li}(\mathrm{I})$ atom coordinated to two tert-butylamine ligands $(\mathrm{Li}-\mathrm{N}=204.5(5) \mathrm{pm})$. Almost in the plane of the $\mathrm{Li}_{2} \mathrm{Br} 2$ ring, two $\mathrm{Sn}_{2} \mathrm{Br}_{2}\{\mathrm{NBu}(\mathrm{H})\}_{2}$ moities are linked through $\mathrm{Br}-\mathrm{Sn}$ bonds, forming a layer of $\mathrm{Sn}, \mathrm{Li}$ and

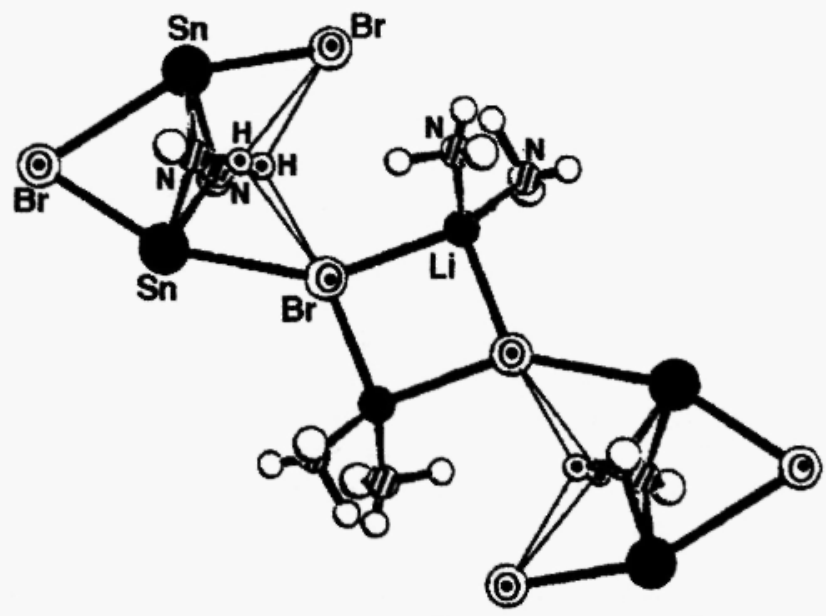

Fig.11. Structure of $\left.\operatorname{Sn}\left\{\mathrm{N}(\mathrm{B}) \mathrm{Bu}^{{ }^{\prime}}\right\}_{2} \mathrm{Br}_{2}\right\}_{2} \mathrm{Lin}_{2} \mathrm{Br}_{2}\left(\mathrm{Bu}^{\prime} \mathrm{NH} 2\right)_{4}[211]$ 
$\mathrm{Br}$ atoms. A colourless $\mathrm{Sn}_{4} \mathrm{Al}_{2}$ cluster [212] contains a central $\mathrm{Sn}_{4} \mathrm{~N}_{4}$ cube $(\mathrm{Sn}-\mathrm{Sn}=323.6(2) \mathrm{pm})$. Two of the $\mathrm{Sn}$ (II) atoms are bonded to an $\mathrm{AlCl}_{3}$ group $\left(\mathrm{Sn}-\mathrm{Al}=278(1) \mathrm{pm}\right.$ ), giving two types of $\mathrm{Sn}$ (II) environment, $\mathrm{SnN}_{3}$ and $\mathrm{SnN}_{3} \mathrm{AI}$. Two colourless $\mathrm{Sn}_{4} \mathrm{In}_{2}$ clusters [213] are isostructural with a central $\mathrm{In}_{2} \mathrm{~N}_{2}$ ring, with each In atom coordinated also to a $\mathrm{CI}$ atom and a methyl or ethyl group. The $\mathrm{N}$ atoms also carry $\mathrm{SnMez}$ groups (average $\mathrm{Sn}-\mathrm{N}$ $=213 \mathrm{pm}$ ), and each $\mathrm{Sn}$ atom is quasi-tetrahedral $\left(\mathrm{SnC}_{3} \mathrm{~N}\right)$. A colourless $\mathrm{Sn}_{4} \mathrm{Sb}_{2}$ cluster [213] has the $\mathrm{Sb}$ atoms bonded at 287.6(1) pm, and each carries two $\mathrm{SnMe}_{3}$ moities with an average $\mathrm{Sn}-\mathrm{Sb}$ bond distance of 279.7(1) $\mathrm{pm}$. This cluster was studied by other workers [214] and at both room temperature and 153(3) K. There are also transition metal derivatives, for example colourless $S_{4} n_{42}$ cluster [215] which contains three four member rings that form a ladder-type structure $\left(\mathrm{Sn}_{4} \mathrm{O}_{4}\right)$. Here, two $\mu_{3}-\mathrm{O}$ and $\mu \mathrm{OH}$ groups serve as bridges between $\mathrm{Sn}$ (II) atoms. Each $\mathrm{OReO}_{3}$ unit is bonded to outer $\mathrm{Sn}$ (IV) atoms, which carry two terminal phenyl groups to complete a distorted trigonal bipyramidal geometry. The Re "atoms have distorted tetrahedral geometry. The structure of a green $\mathrm{Sn}_{4} \mathrm{Ni}$ cluster [216] contains a six membered ring $\left[\mathrm{NiCNSnOSnNC}_{2}\right.$ with a OH group acting as a bridge between a pair of Sn(IV) atoms. Each Sn(IV) atom is also coordinated to three methyl groups creating a distorted trigonal-bipyramidal geometry $\left(\mathrm{SnC}_{3} \mathrm{ON}\right)$ with $\mathrm{O}$ and $\mathrm{N}$ atoms at the apices. The $\mathrm{Ni}(\mathrm{TV})$ atoms each carny $\mathrm{CN}$ groups to complete $\mathrm{NiC}_{4}$ stereochemistry. In yellow $\mathrm{Sn}_{4} \mathrm{Ru}_{2}$ cluster [217] two ( $\left.\mathrm{Me} 3 \mathrm{Sn}\right)_{2} \mathrm{Ru}(\mathrm{CO})_{3}$ groups are linked by two bridging Me3Sn groups. The $\mathrm{Rn}_{2} \mathrm{Sn}_{3}$ bridge plane contains a crystallographic centre of symmetry. Each $\mathrm{Ru}$ atom is bonded to six atoms $\left(\mathrm{RuC}_{3} \mathrm{Sn}_{3}\right)$ and the two octahedra share an edge to form the dimeric unit. The $\mathrm{Sn}-\mathrm{Ru}$ distances are 263.8(2), 268.6(2) and 269.4(2) pm. The $\mathrm{Ru}-\mathrm{Ru}$ distance is $311.6(3) \mathrm{pm}$. Two pairs of $\mathrm{Sn}$ atoms, $\mathrm{SnC}_{2} \mathrm{Ru}_{2}$ and $\mathrm{SnC}_{3} \mathrm{Ru}$, have a quasi-tetrahedral arrangement. A dark green $\mathrm{Sn}_{4} \mathrm{Rh}_{2}$ cluster [218] consists of two edge shared octahedral rhodium centres linked by two bridging $\mathrm{SnCl}_{2}$ groups. The octahedral coordination about each rhodium is completed by three terminal 2,6-xylyl isocyanate ligands (average $\mathrm{Rh}-\mathrm{C}=$ 199(1) pm and one terminal $\mathrm{SnCl}_{3}$ group trans to the $\mathrm{SnCl}_{2}$ bridges. The $\mathrm{Sn}_{4} \mathrm{Rh}_{2}$ arrangement of metal atoms is approximately planar. A crystallographic centre of inversion is found in the centre of the $\mathrm{Rh}_{2} \mathrm{Sn}_{3}$ rhombus. The shorter Sn-Rh bond length to the $\mathrm{SnCl}_{3}$ ligand is $261.6(1) \mathrm{pm}$, and the longer to the $\mathrm{SnCl}_{2}$ bridge is 264.5(1) $\mathrm{pm}$. This reflects a greater degree of $\mathrm{Sn}-\mathrm{Rh} \pi$-bonding in the former. The $\mathrm{Sn}$ atoms have quasi-tetrahedral geometry. All of the results are comparable

\subsection{Three Sn plus Three $M$}

The $\mathrm{S}_{13} \mathrm{M}_{3}$ core is found in eight clusters, all with transition metal atoms. The structure of $\mathrm{Sn}_{3} \mathrm{Mn}_{3}$ cluster [219] has a six membered metallocycle of alternate $\mathrm{Sn}$ and $\mathrm{Mn}$ atoms with average $\mathrm{Sn}-\mathrm{Mn}$ bond distances of $258.8(5) \mathrm{pm}$. The endocyclic ring angle at $\mathrm{Mn}$ is $90.5(2)^{\circ}$ and at $\mathrm{Sn}$ is $141.6(2)^{\circ}$. A yellow $\mathrm{Sn}_{3} \mathrm{Fe}_{3}$ cluster [220] contains a bicyclic $\mathrm{Sn}_{3} \mathrm{Se}_{4}$ framework with Sn-Se-Sn angles of $94.6(1)^{\circ}$ (four membered ring) and $110.5(1)^{\circ}$ (six membered ring). Each $\mathrm{Sn}$ (II) atom also carries an $\mathrm{Fe}(\mathrm{CO})_{2} \mathrm{Cp}$ moiety with an average $\mathrm{Sn}$-Fe bond distance of 249.2(2) pm. Each tin atom is quasi-tetrahedral, $\mathrm{SnSe}_{3} \mathrm{Fe}$ and $\mathrm{SnSe}_{2} \mathrm{ClFe}$. There are two red and one orange $\mathrm{Sn}_{3} \mathrm{Ru}_{3}$ clusters [96] which are isostructural. The $\mathrm{Ru}$ triangle and three $\mathrm{Sn}$ atoms form a planar array with average $\mathrm{Sn}-\mathrm{Ru}$ bond distance of $271 \mathrm{pm}(288.7(2)$ to $297.7(2) \mathrm{pm})$. One of the clusters contains two crystallographically independent molecules, differing by degree of distortion (Table 4). In all clusters the Sn atoms are quasitetrahedral $\left(\mathrm{SnC}_{2} \mathrm{Ru}_{2}\right)$. In one $\mathrm{Sn}_{3} \mathrm{Pd}$ cluster [221] the metal atoms form a planar array similar to the previous cases [96]. The mean Sn-Pd bond distance of $262 \mathrm{pm}$ is about $9 \mathrm{pm}$ shorter than that found in SnzRuz clusters [96]. Each $\mathrm{Sn}$ atom is in a distorted tetrahedral environment $\mathrm{SnN}_{2} \mathrm{Pd}_{2}$. The remaining $\mathrm{Sn}_{3} \mathrm{M}_{3}$ clusters $(\mathrm{M}=\mathrm{Os}$ [154] or Pt [221]) are isostructural with the $\mathrm{Ru}$ and Pd analogues discussed above. The mean Sn-Os and Sn-Pt bond distances are 267 and $262 \mathrm{pm}$, respectively. The mean Os-Os distance is $297.4(1) \mathrm{pm}$, and mean Sn-Pt distance is $276.3(1) \mathrm{pm}$. In this subsection, six examples are isostructural $[96,154,221]$ with a triangle of $M$ atoms and the three Sn atoms forming a planar array. The mean Sn-M bond distance increases in the order: 262 $\mathrm{pm}(\mathrm{Pd}, \mathrm{Pt})<267 \mathrm{pm}(\mathrm{Os})<271 \mathrm{pm}(\mathrm{Ru})$. The mean M-M bond distance increases in the order: $276 \mathrm{pm}(\mathrm{Pt})<$ 281 pm (Pd) $<294$ pm (Ru) $<297$ pm (Os).

\subsection{Two Sn plus Four $M$}

The $\mathrm{Sn}_{2} \mathrm{M}_{4}$ clusters contain a non-transition metal example where $\mathrm{M}$ is aluminium [222]. This has an eight membered ring $\mathrm{Al}_{2} \mathrm{Sn}_{2} \mathrm{Cl}_{4}$ with two $\mathrm{Sn}$ (II) centres linked by two $\mathrm{AlCl}_{4}$ moities. Each $\mathrm{Sn}$ (II) atom is $\eta^{\circ}$ bonded to a hexamethylbenzene ring at a distance of $245 \mathrm{pm}$.

The other Sni M clusters all involve transition metal heterometal atoms. Five of these $\left(M=M_{0}\right.$ [223], $\mathrm{Fe}[223,230]$, $\mathrm{Co}[232])$ clusters contain planar $\mathrm{Sn}_{3} \mathrm{X}_{2}$ rings $(X=\mathrm{Te}[223], \mathrm{Se}[230,232]$ or $\mathrm{S}$ [237]. Four $\mathrm{Mcp}(\mathrm{CO})_{2}[223,230]$ or $\mathrm{Co}(\mathrm{CO})_{4}$ moities [232] are connected in pairs to $\mathrm{Sn}$ atoms to complete a quasitetrahedral environment $\left(\mathrm{SnX}_{2} \mathrm{M}_{2}\right)$. As the covalent radius of $\mathrm{M}$ increases so does the mean value of the $\mathrm{Sn}-\mathrm{M}$ distance, for example: $258.7(2) \mathrm{pm}(\mathrm{Fe}, 120 \mathrm{pm})<259.2(2) \mathrm{pm}(\mathrm{Co}, 126 \mathrm{pm})<288.7 \mathrm{pm}(\mathrm{Mo}, 145 \mathrm{pm})$.

There are two orange $\mathrm{Sn}_{2} \mathrm{~W}_{4}$ clusters $[224,225]$ which contain $\left[\mathrm{Sn}_{2}\left(\mathrm{WX}_{4}\right)_{4}\right]^{-4}$ anions $(X=\mathrm{S}[224]$ or $\mathrm{Se}$ 
[225]). The anion contains two central SnY 6 octahedra coupled via a common edge. These octahedra are bonded to four non-equivalent WY $\mathrm{Y}_{4}$ tetrahedra via common edges. Two of the $\mathrm{WY}_{4}^{2}$ anions are coordinated as bidentate ligands to the $\mathrm{Sn}$ (II) atom and retain two free $\mathrm{S}$ atoms. The other $\mathrm{WY}_{4}{ }^{-7}$ anions coordinate as tridentate ligands with only one free $S$ atom. These tridentate WY4 ligands contain a triply bonded $S$ atom.

There are six Sn2Mn4 clusters [166,226-229]. Molecules of $\mathrm{H}_{2} \mathrm{Sn}_{2}\left\{\mathrm{Mn}(\mathrm{CO})_{5}\right\}_{4}$ [226] have $\mathrm{C}_{2}$ symmetry with a tin-tin axis $(\mathrm{Sn}-\mathrm{Sn}=289.4(5) \mathrm{pm})$. The managanese atoms are in an octahedral environment comprised of five $\mathrm{CO}$ groups and one $\mathrm{Sn}$ atom. Each tin atom is in a distorted tetrahedral environment (SnMn2Sn') with the Sn-Mn bond distances of 267.5(5) pm and 273.0(5) pm, respectively. Another two Sn2Mn4clusters [166,227] are isostructural. The central fragment contains a planar $\mathrm{Mn} 2 \mathrm{Sn}_{2}$ rhombus with a $\mathrm{Mn}-\mathrm{Mn}$ bond (309.1(1) pm [166] and 308.6(1) pm [227] across the ring, and Sn-Mn bon distances of 262.0(1) and 262.7(1) pm [166] or 262.5(1) and 263.0(1) pm [227]. The Sn(IV) atoms each have a terminal bond to $\mathrm{Cl}$ [166] or $\mathrm{Br}$ [227], and a $\mathrm{Mn}(\mathrm{CO})$ s ligand (Sn-Mn $=262.6(1) \mathrm{pm}$ [166] or $263.4(1) \mathrm{pm}$ [227]), to create a distorted tetrahedral environment. Two red-brown $\mathrm{Sn}_{2} \mathrm{Mn}_{4}$ clusters [228] are isostructural and composed of an $\mathrm{Sn}_{2}^{-2}$ moiety coordinated linearly end-on to two Mncp'(CO) 2 units. In addition, the tin centres are bridged by two $\mu-\mathrm{Mn}\left(\mathrm{cp}^{\prime}(\mathrm{CO})_{2}\right.$ units and a $\mu-\mathrm{Cl}$ ligand. The resulting structure thus resembles a [1.1.1] propellane scaffolding with a mean Sn-Sn distance of 285.2(2) $\mathrm{pm}$. The Sn-Mn bond distances range from 250.8(14) to 276.0(3) pm. A dark red $\mathrm{Sn}_{2} \mathrm{Mn}_{4}$ cluster [229] has a central Sn-Sn (288.5(1) pm unit, with each Sn tetrahedrally surrounded by two $\mathrm{Mn}(\mathrm{CO})$ s groups and one $\mathrm{Br}$ atom. The average Sn-Mn bond distance is $273 \mathrm{pm}$, and the $\mathrm{Sn}-\mathrm{Sn}-\mathrm{Mn}$ angles range from $113.26(3)^{\circ}$ to $117.26(3)^{\circ}$.

There are three coloured $\mathrm{Sn}_{2} \mathrm{Fe}_{4}$ clusters $[223,230,231]$, two of which are isostructural and have been discussed above $[223,230]$ together with corresponding Sn2 Cou structures [232]. The third [231] contains a centrosymmetric $\mathrm{Sn}_{2} \mathrm{O}$ 2 dimer with unsymmetrically bridged $\mathrm{OH}$ groups $(\mathrm{Sn}-\mathrm{O}=202(1)$ and 223(1) pm). Each tin carries two ( $\mu$ - $\left.\mathrm{C}_{5} \mathrm{H}_{3}-2-\mathrm{CH}_{2} \mathrm{NMe}_{3}\right) \mathrm{Fecp}$ moities and a terminal $\mathrm{OH}$ group, completing a distorted trigonalbipyramidal arrangement $\left(\mathrm{SnO}_{3} \mathrm{C}_{2}\right)$ with two of the $\mathrm{O}$ atoms at apical positions $(\mathrm{O}-\mathrm{Sn}-\mathrm{O}=158.4(5) \mathrm{pm})$.

Two yellow $\mathrm{Sn}_{2} \mathrm{Re}_{4}$ clusters $[233,234]$ are also isostructural. The central fragment contains a planar $\mathrm{Re}_{2} \mathrm{Sn}_{2}$ rhombus with a trans-annular Re-Re bond of $316.0(2) \mathrm{pm}$ [233] or $317.6(1) \mathrm{pm}$ [234]. A CI [233] or I [234] plus a $\operatorname{Re}(\mathrm{CO})_{4}\left(\mathrm{PPh}_{3}\right)$ moiety are bonded to $\mathrm{Sn}(\mathrm{IV})$ in trans positions with respect to the ring plane. The mean Sn-Re bond distances are almost equal at 274 and 275 pm, respectively. Each Sn(IV) atom is quasitetrahedrally coordinated, $\mathrm{SnXRe}$.

A gold-yellow $\mathrm{Sn}_{2} \mathrm{Au}_{4}$ cluster [235] consists of a tetrahedral Au core with opposite edges bridged by $\mathrm{SnCl}_{3}$ groups. The molecule has a crystallographically imposed $\mathrm{C}_{2}$ symmetry axis. The $\mathrm{Au}-\mathrm{Au}$ bond angles range from 263.4(5) to $281.28(5) \mathrm{pm}$, the shorter valuses being those where the Au atoms are edge-bridged by the $\mathrm{SnCl}_{3}$ groups. The Au-Au-Au bond angles range from $56.34(9)^{\circ}$ to $62.72(1)^{\circ}$, showing a slight departure from regular tetrahedral geometry. Th Sn-Au bond lengths are 281.5(7) and 297.3(8) pm. Each Sn atom has a pseudo-trigonal bibyramidal arrangement $\left(\mathrm{SnCl}_{3} \mathrm{Au}\right)$.

\subsection{Sn plus Five $M$}

The structure of a green $\mathrm{SnFes}$ cluster [236] consists of a $\mathrm{SnFe}_{2}$ triangle linked to an $\mathrm{Fe}_{3}$ triangle via a spiro Sn atom. The mean Fe-Fe distance on the unbridged sides $(270.8(1) \mathrm{pm})$ is about $5 \mathrm{pm}$ longer than the bridged side at $265.8(1) \mathrm{pm}$. The $\mathrm{Fe}-\mathrm{Fe}$ bond of the $\mathrm{Fe}_{2}(\mathrm{CO})_{8}$ group is longest at 287.8(2) pm, and the $\mathrm{Sn}-\mathrm{Fe}$ bond distances range from $253.5(1)$ to $259.0(1) \mathrm{pm}$.

\subsection{Two Sn plus $4(M+M ')$}

There are three examples which have two heterometal atoms present with tin. A bright orange $\mathrm{Sn}_{2} \mathrm{Os} 3 \mathrm{Pt}$ cluster [237] contains a tetrahedral array of two Os, one Pt and one Sn atom. One of the Os atoms bears three $\mathrm{CO}$ groups while the other carries two $\mathrm{CO}$ groups and a trichlorostannate(II) ligand. The Pt atom carries a tricyclohexyl phosphine group and a $\mathrm{CO}$ ligand. The tin atom is bonded to a CI atom and a molecule of diethyl ether. An Os(CO)4 unit bridges the Sn atom and the $\mathrm{Os}(\mathrm{CO})_{3}$ moiety, resulting in an overall edge-bridged tetrahedral metal core. The separations between Os and $\mathrm{Sn}$ range from $260.8(4)$ to $305.8(5) \mathrm{pm}$. A brown $\mathrm{Sn}_{2} \mathrm{~W}_{2} \mathrm{Fe}_{2}$ cluster [238] contains half of the metal atoms, a sodium ion, two diethyl ether molecules and half of a dioxane molecule. The cluster dianion is a centrosymmetric dimer built around a planar $\mathrm{Sn}_{2} \mathrm{Fe}_{2}$ core. Each of the $\mathrm{W}(\mathrm{CO})_{3}$ moities is bound to the $\mathrm{Sn}$ atom of this core. The $\mathrm{SnFe}_{2} \mathrm{~W}$ unit is nearly planar with the tSn atom only 55.4(4) pm out of the $\mathrm{Fe}_{2} \mathrm{~W}$ plane. The Sn-Fe bond distances of 268.1(1) and 269.4(2) pm are shorter than the $\mathrm{Sn}-\mathrm{W}$ bond distance of 279.9(1) pm. The third cluster $\mathrm{Sn}_{2} \mathrm{Fe}_{2} \mathrm{CO}_{2}$ [126] has six metal atoms arranged in a one dimansional chain, $(\mathrm{CO})_{4} \mathrm{FeSn}(\mu-\mathrm{OBu})_{2} \mathrm{Co}(\mu-\mathrm{OBu})_{2} \mathrm{Co}(\mu-\mathrm{OBu})_{2} \mathrm{SnFe}(\mathrm{CO})_{4}$. Each $\mathrm{Sn}$ atom is also coordinated to a terminal OBu group. The $\mathrm{Sn}-\mathrm{Fe}$ bond distance is $248.0(1) \mathrm{pm}$, the Co-Co distance is $297.7(3) \mathrm{pm}$, but the Co-Sn distance of 316.5(1) pm indicates no metal-metal bond

The data in Table 4 shows that the $\mathrm{Sn}$ atom in the hexameric compounds is mostly in a quasi- 


\begin{tabular}{|c|c|c|c|c|c|c|c|c|c|c|c|c|}
\hline 㟧 & ) & & T & & $\stackrel{\infty}{\stackrel{\nu}{\circ}}$ & & ஜ્లి & & & & & \\
\hline$\sum_{i}$ & 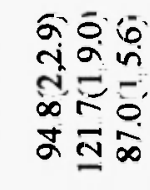 & 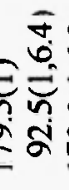 & & 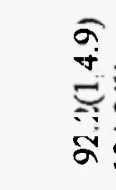 & 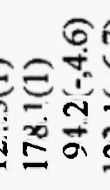 & 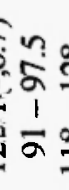 & 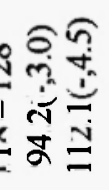 & $\begin{array}{l}0 \\
0 \\
0.0 \\
\dot{0}\end{array}$ & 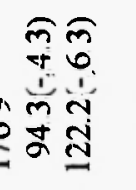 & 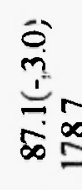 & 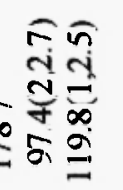 & $\begin{array}{l}\frac{1}{3} \\
0 \\
8\end{array}$ \\
\hline$\sum$ & 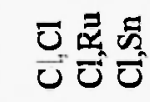 & $\begin{array}{l}\text { मू } \\
\text { हี }\end{array}$ & $\frac{E_{0}}{\square}$ & $\begin{array}{l}\text { ผี } \\
\text { ผี }\end{array}$ & $\bar{U}$ & $\bar{\tau}$ & 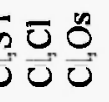 & है & $\begin{array}{l}\vec{v} \stackrel{0}{0} \\
\bar{v}\end{array}$ & $\begin{array}{l}\frac{5}{\sigma} \\
\end{array}$ & $\begin{array}{l}\vec{U} \\
\vec{U}\end{array}$ & $\begin{array}{l}\text { हี } \\
\text { मี }\end{array}$ \\
\hline 롤롤 & 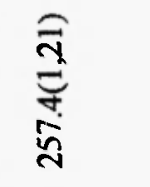 & & 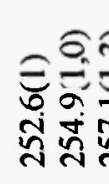 & & $\frac{8}{8}$ & & 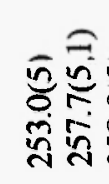 & & 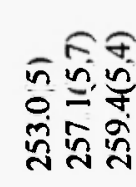 & & 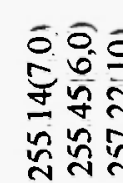 & \\
\hline 它主 & $\vec{z}$ & & 중 & & $\hat{o}$ & & 8 & & 8 & & $\Xi$ & \\
\hline 昱畐 & 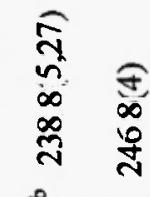 & & 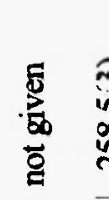 & 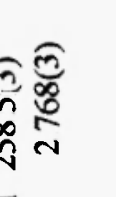 & $\begin{array}{l}6 \\
\frac{1}{2} \\
\frac{1}{2}\end{array}$ & 命 & 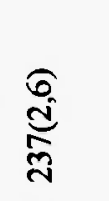 & 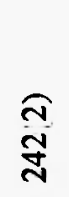 & $\begin{array}{l}\widetilde{n} \\
\tilde{d} \\
\tilde{d} \\
\tilde{n}\end{array}$ & 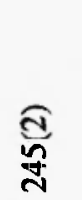 & 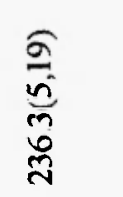 & \\
\hline & $\stackrel{0}{U}$ & & $\mathbf{z}$ & بِ & $\bar{\sigma}$ & $\bar{v}$ & $\bar{U}$ & $\bar{U}$ & $\bar{U}$ & $\bar{v}$ & ن & \\
\hline 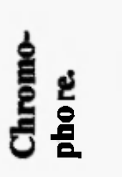 & 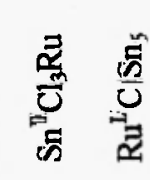 & & 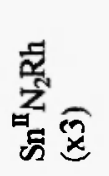 & 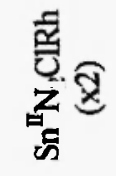 & 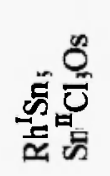 & 望 & 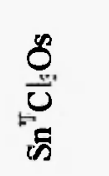 & $\underset{\mathscr{n}}{\mathscr{D}}$ & 兽 & $\begin{array}{l}\tilde{\mathscr{E}} \\
\tilde{0} \\
\tilde{0}\end{array}$ & $\underset{D}{\overrightarrow{0}}$ & मี \\
\hline$\sigma_{0} \sum_{0}$ & & & & & & $\hat{\tilde{N}}$ & & & & & 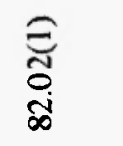 & \\
\hline 馬亘㗐 & 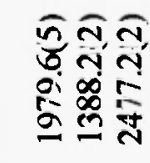 & & 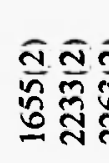 & & 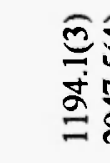 & $\begin{array}{l}\frac{\infty}{\cos } \\
\frac{\operatorname{s}}{2}\end{array}$ & 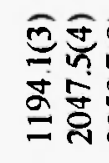 & & & & 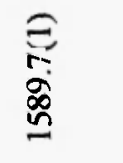 & \\
\hline 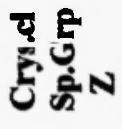 & $\frac{\mathscr{J}}{\tilde{E}}$ & & ๘ & & $E$ & & $\Xi \underset{\pi}{E}$ & & & & of & \\
\hline 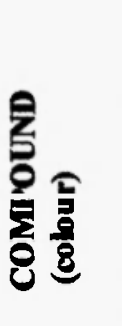 & 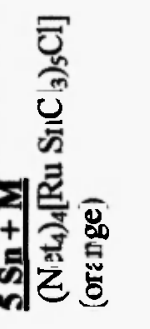 & & 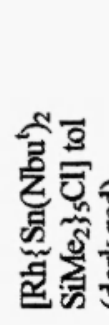 & & $\begin{array}{l}8 ? \\
\text { हैं }\end{array}$ & 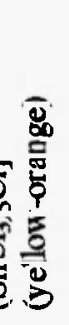 & 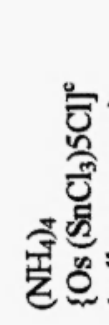 & & & & 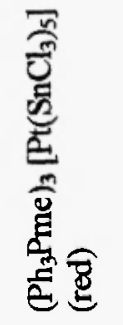 & \\
\hline
\end{tabular}


$\bar{\pi}$

$\stackrel{\text { ส }}{2}$

$\stackrel{m}{3}$

$\stackrel{n}{N}$

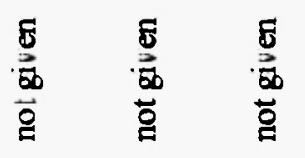

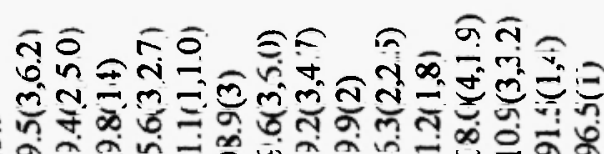

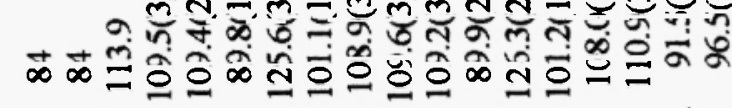

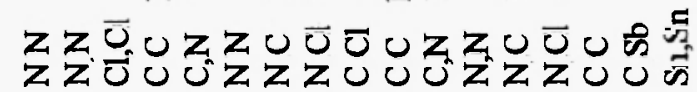

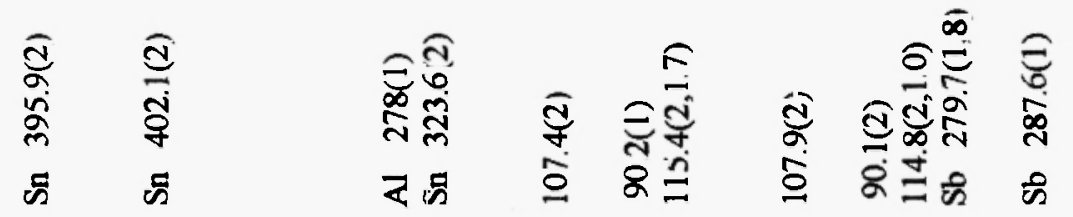

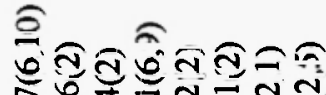
กำ

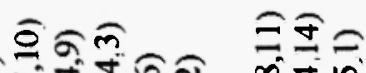

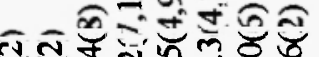

पर्व

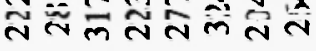

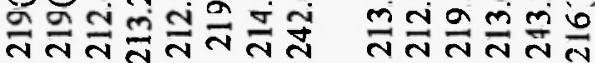

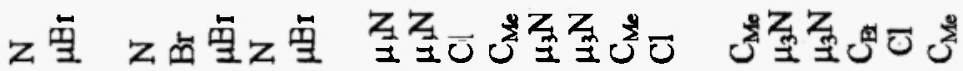

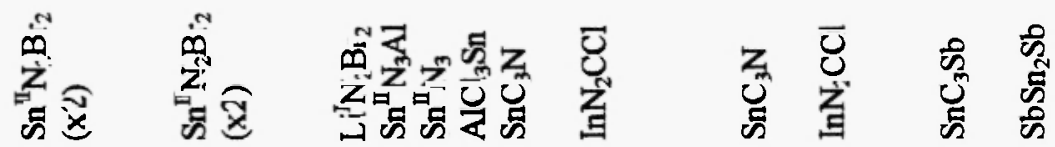

อิกิก

$\exists$

केतळ

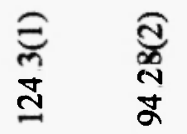

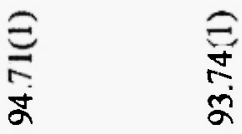

ลิ人̄

$\forall \bar{c}$

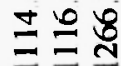

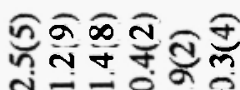

‥

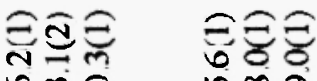

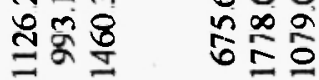

घค.

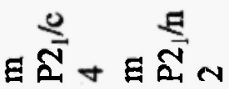

ะล

ล $\stackrel{\Sigma}{\Sigma}$
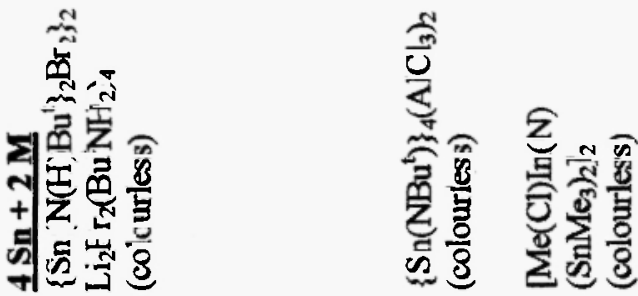

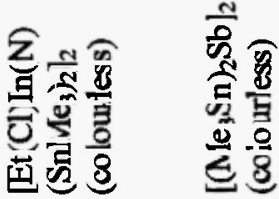


$\frac{\pi}{\Delta}$

$\frac{n}{n}$

$\frac{1}{\pi}$

咅

क’

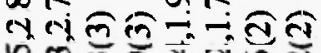

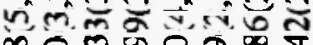

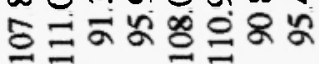

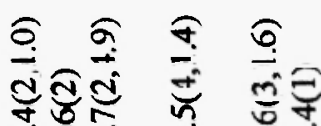

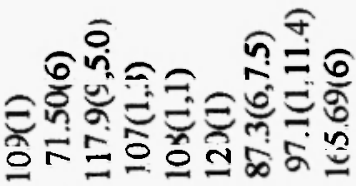

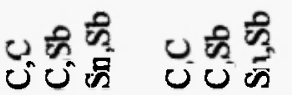

ॠळ

0 0 000

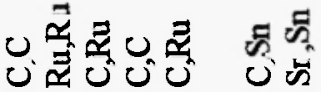

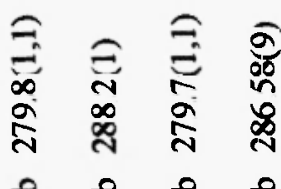

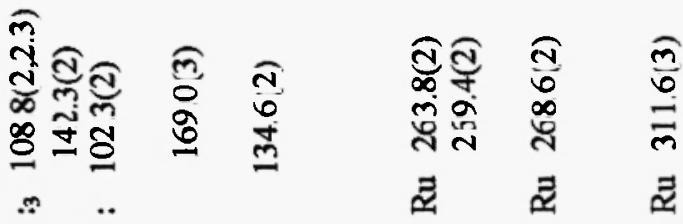

ஜ हे ह ஜ

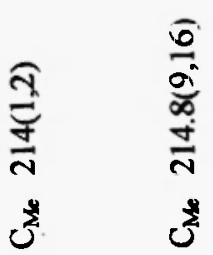

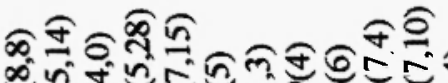

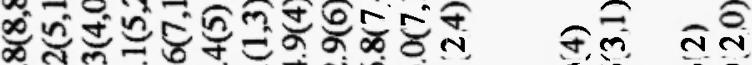

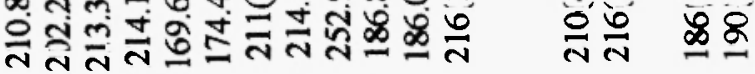

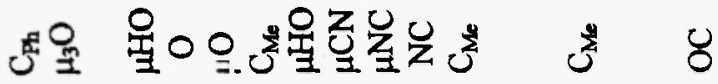

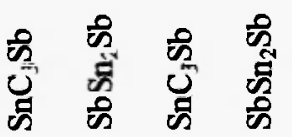

仓े

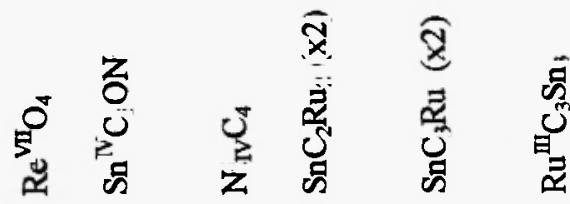

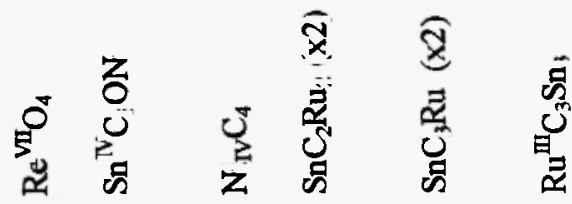

ปั
ปั่

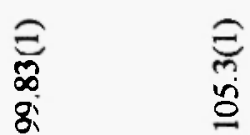

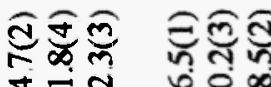

सत्र

를

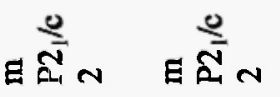

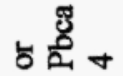

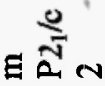

$E \stackrel{\frac{e}{a}}{a^{2}} N$
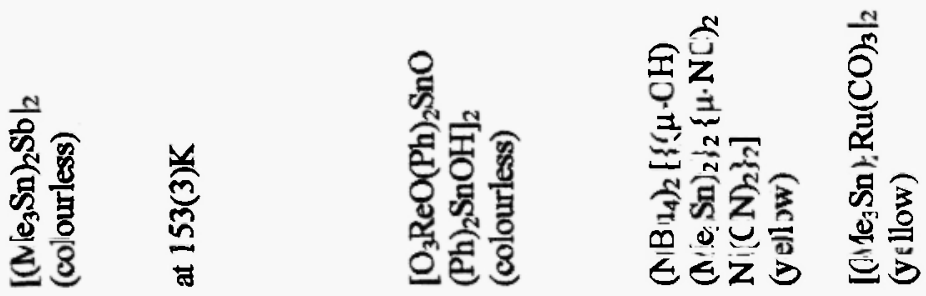
$\stackrel{\infty}{\text { กे }}$ สิ

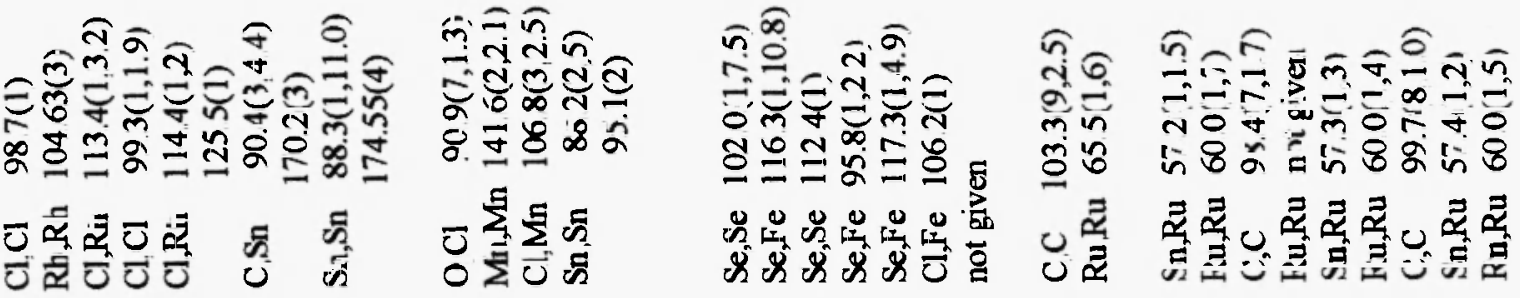

\begin{tabular}{|c|c|c|c|}
\hline 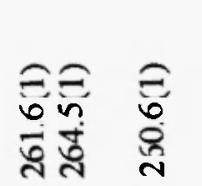 & 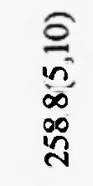 & 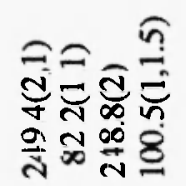 & 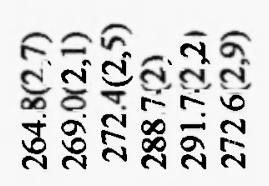 \\
\hline$\vec{x}$ & 表 & 哇 & $\vec{z} \quad \vec{x} \quad \vec{z}$ \\
\hline
\end{tabular}

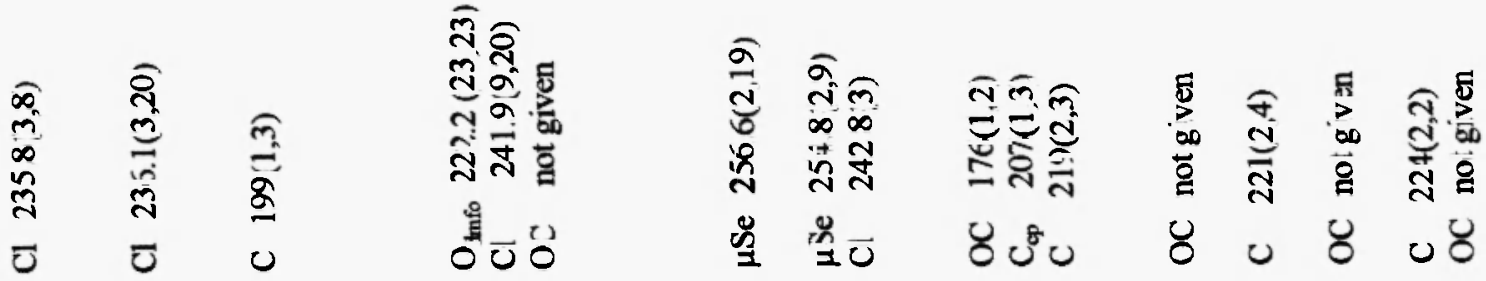

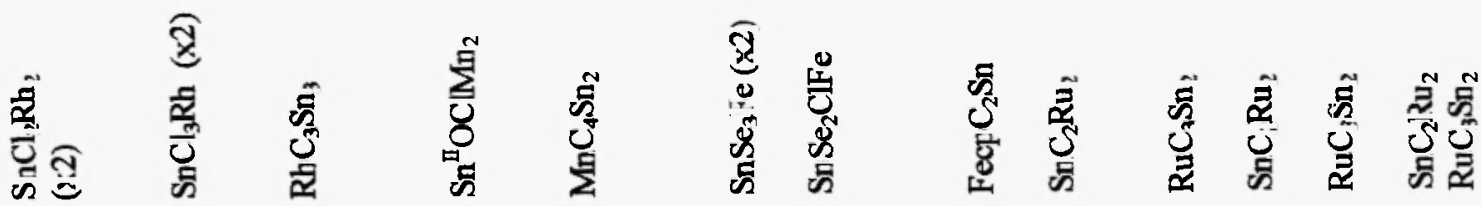

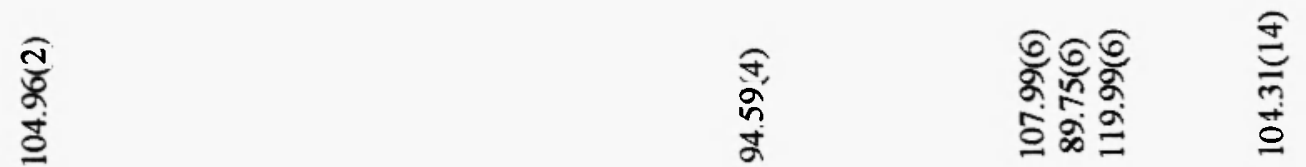

可高

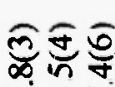

这

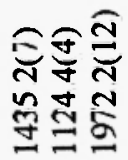

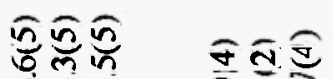

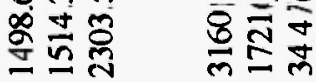

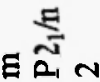

๖

空

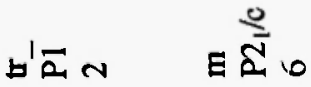
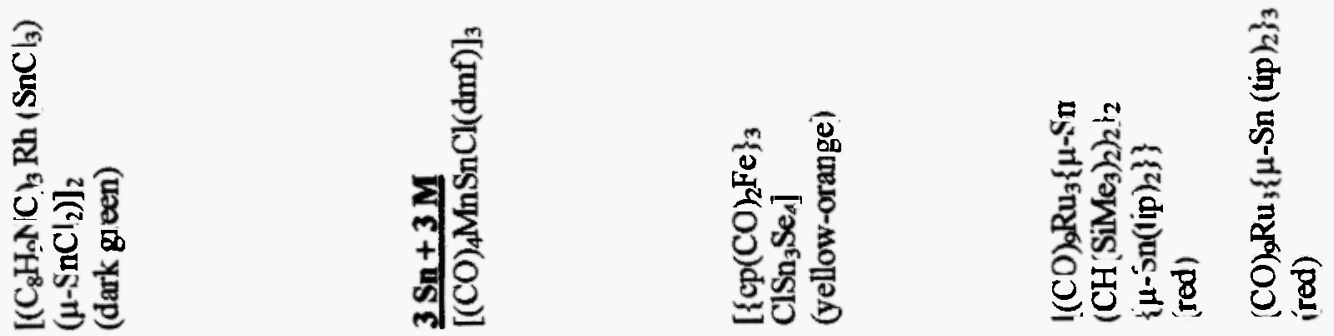
$\%$

กิ ถับ

$\overline{\text { ส }}$

สี

สี

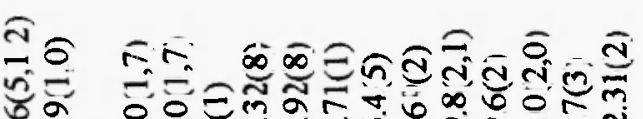

尚

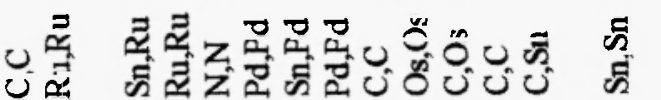

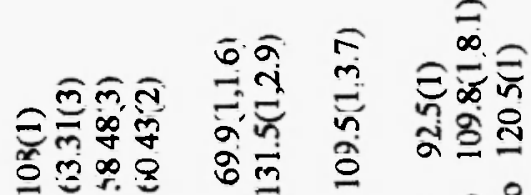

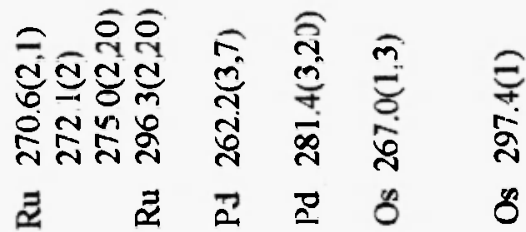

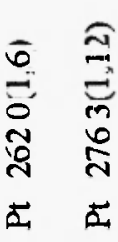

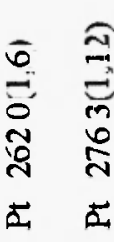

귱

恶离

$\sum^{0}$

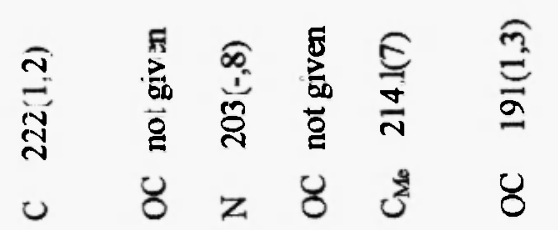

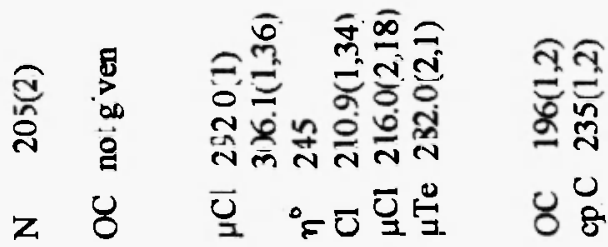

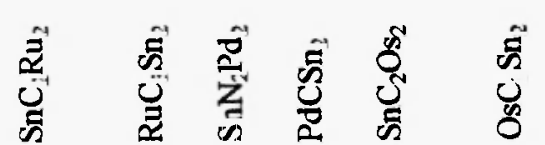

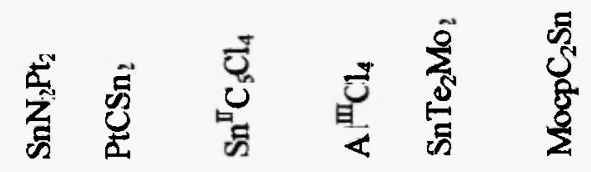

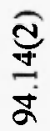

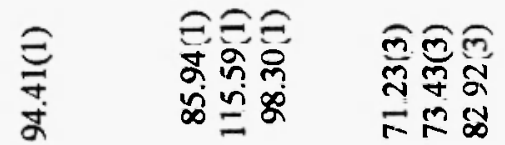

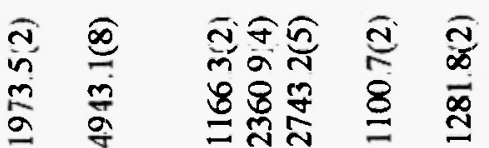

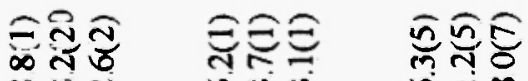

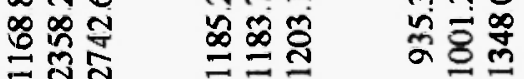

ํํำ

घ
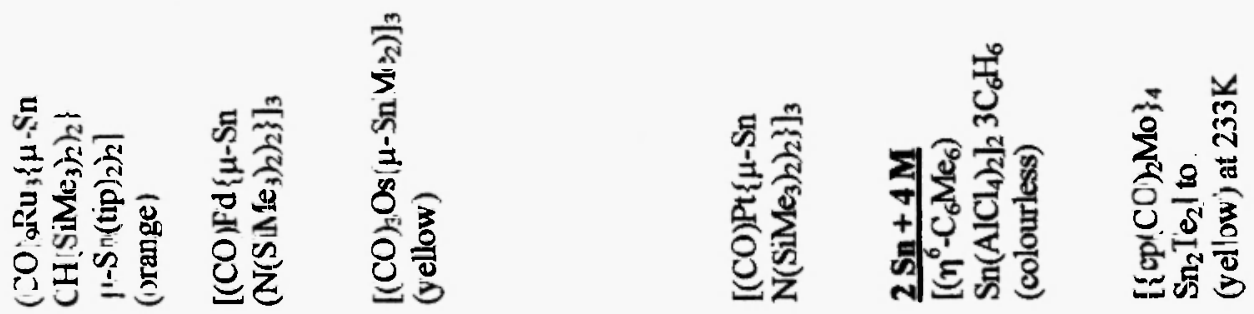
$\stackrel{\text { ה }}{+}$

สิ สั

$\dddot{8}$

สิ

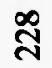

$\stackrel{\text { స్ }}{*}$

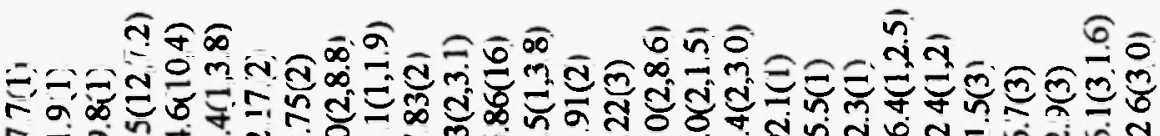

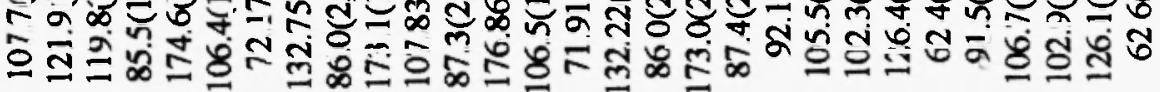

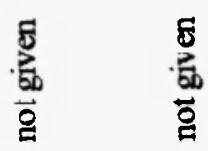

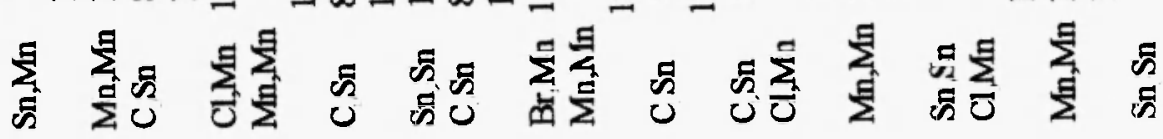

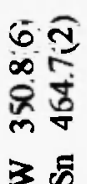

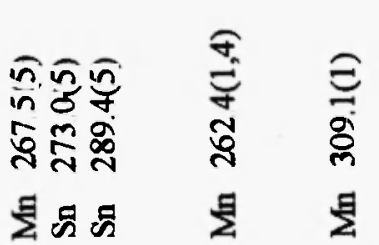

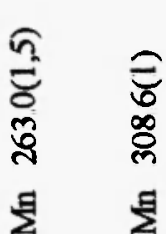

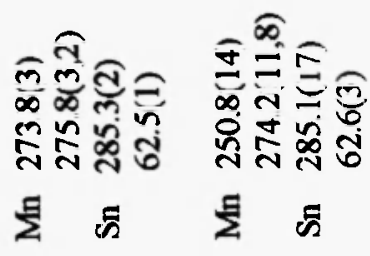

$\frac{1}{\infty}, \frac{0}{0} \overline{0}-\widehat{m}$

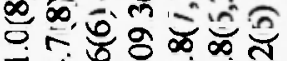

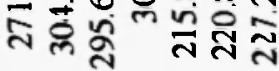

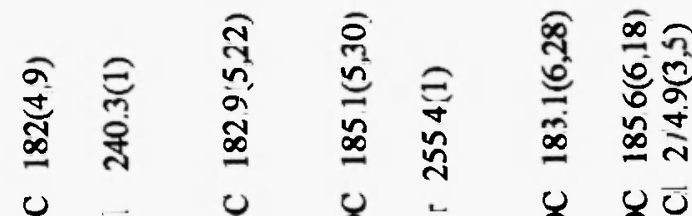

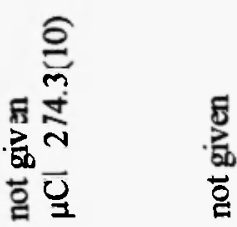

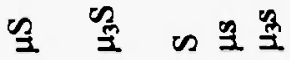

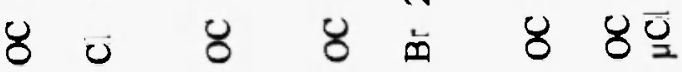

范

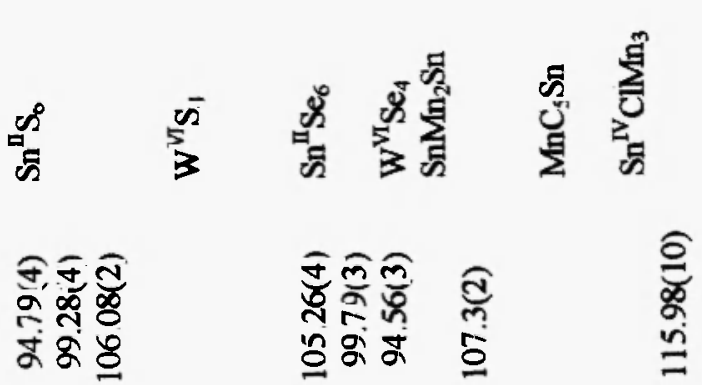

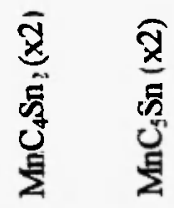

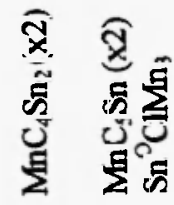

范点

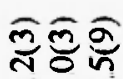

守导商

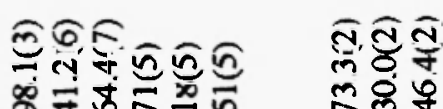

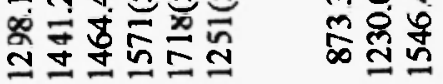

r.

흥

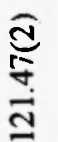

$\stackrel{4}{3}$

的-

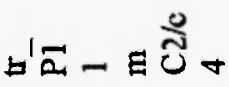

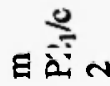

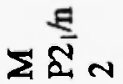

छ
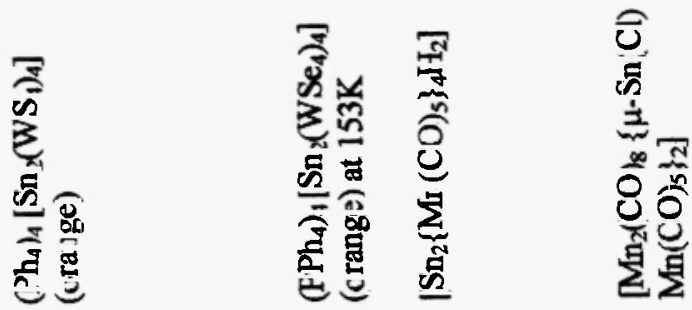

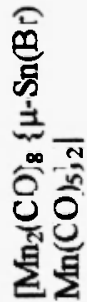

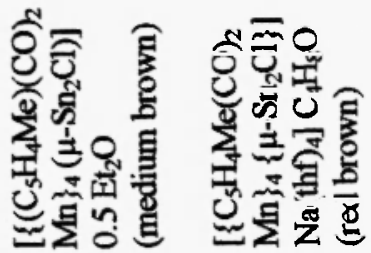


กิ

ֻำ

สิ

$\bar{c}$

กิ

กิ

$\ddot{\text { กิ }}$

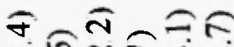

กิ่

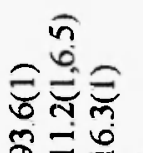

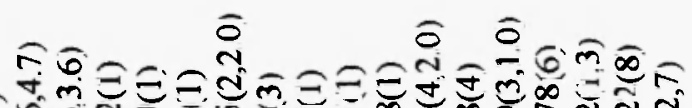

근

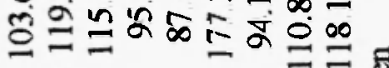

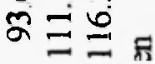

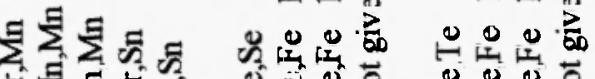

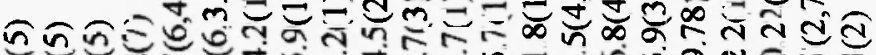

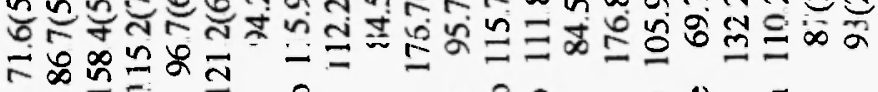

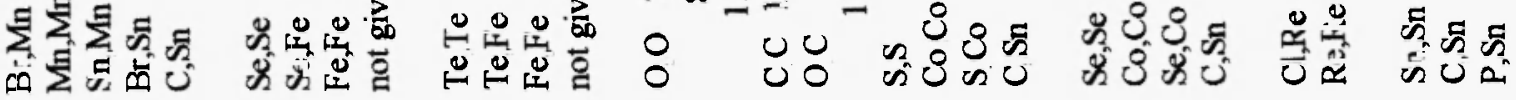

\begin{tabular}{|c|c|c|c|c|c|c|}
\hline 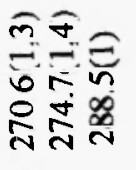 & 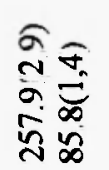 & 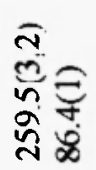 & $\begin{array}{l}\frac{\infty 0}{y} \\
80 \\
0\end{array}$ & 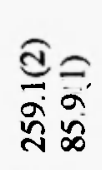 & 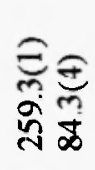 & 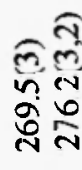 \\
\hline ह & : & D & 오 & 8 & 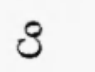 & $\mathscr{2}$ \\
\hline
\end{tabular}

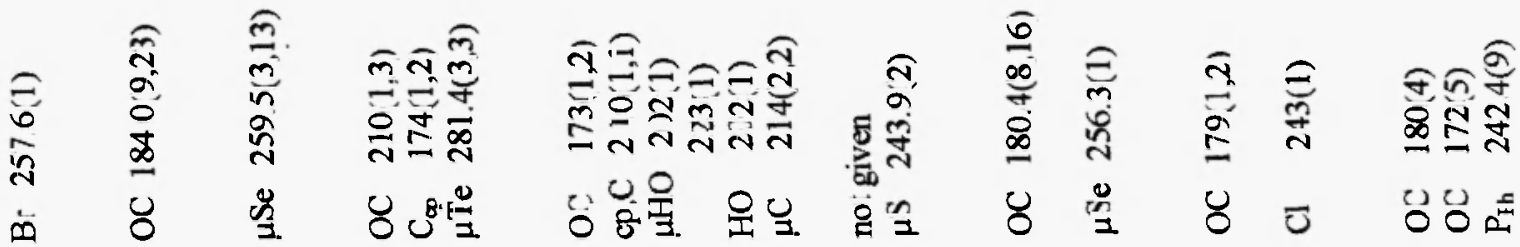

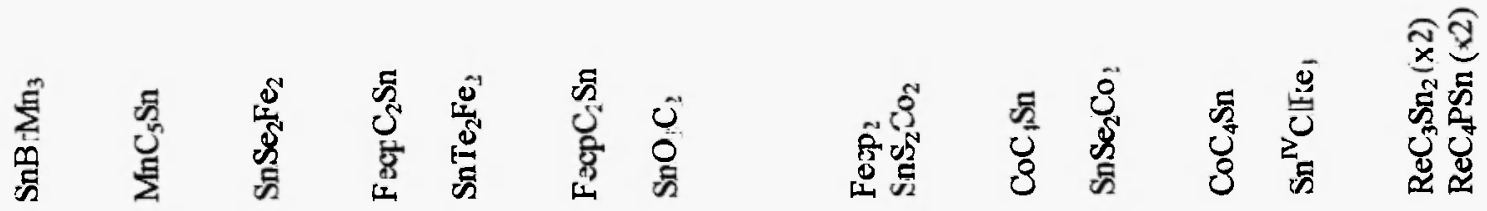

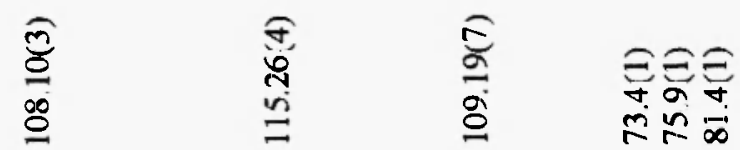

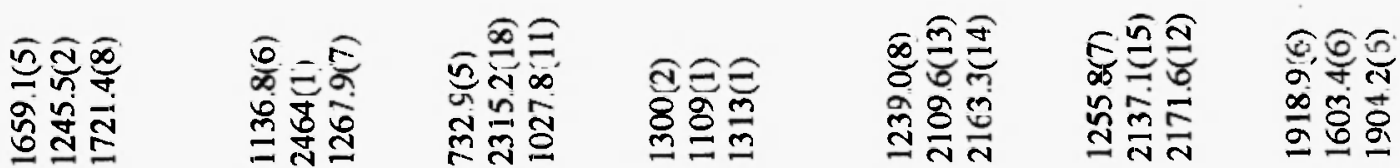

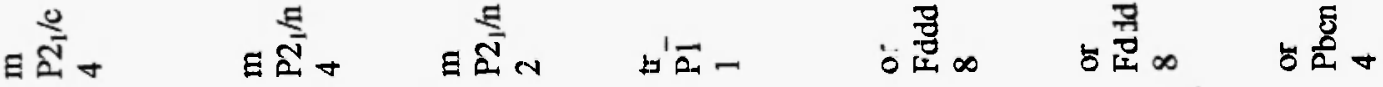
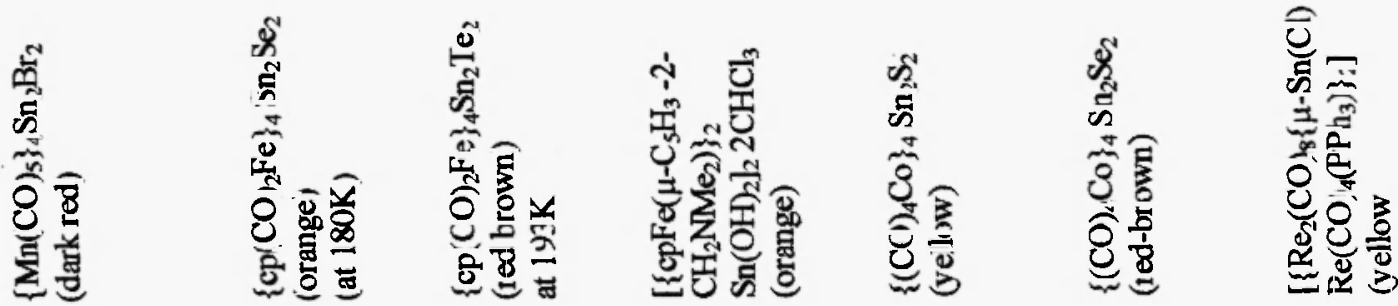


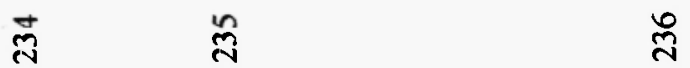

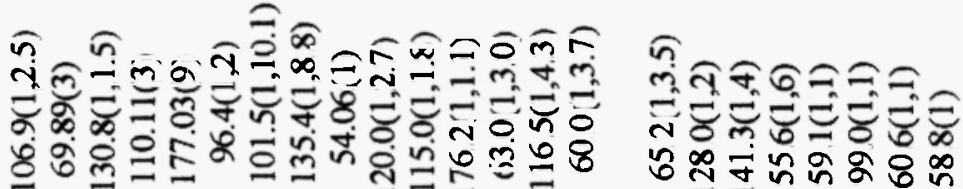

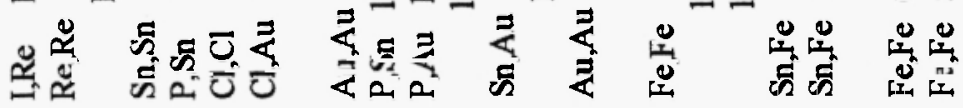

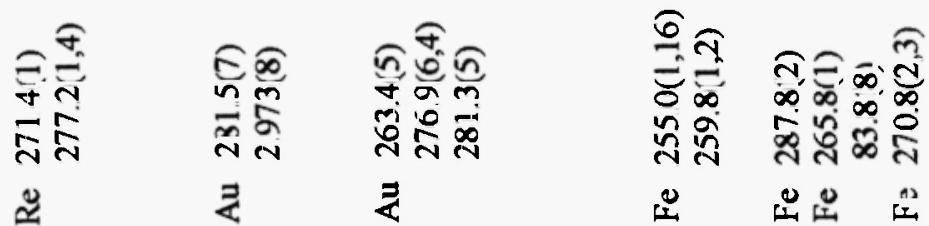

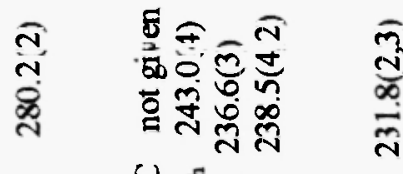

ฐ

कiv ab of क

㖓

ชర్ర

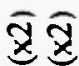

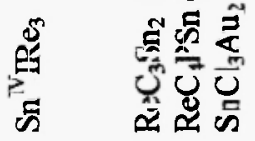

芠

$\widehat{\widetilde{x}} \widehat{\hat{x}}$

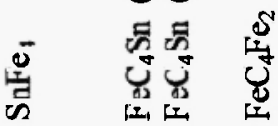

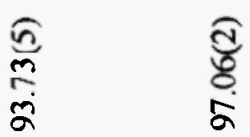

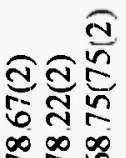

등호을

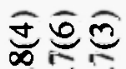

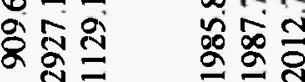

ฮูํำ

大。

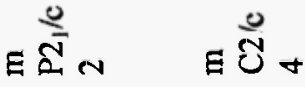

$\tan$

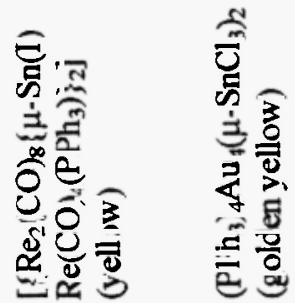

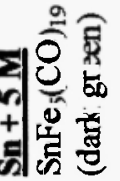


$\tilde{\approx}$

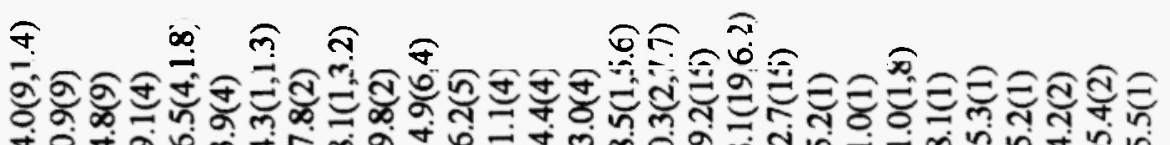

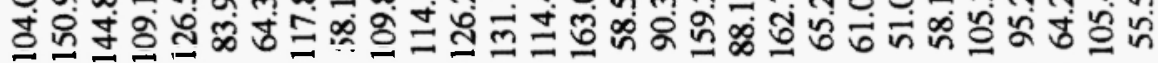

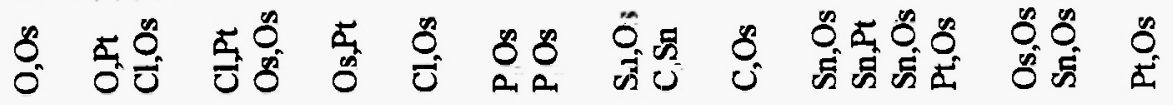

气ูn

ชู่

8 ז

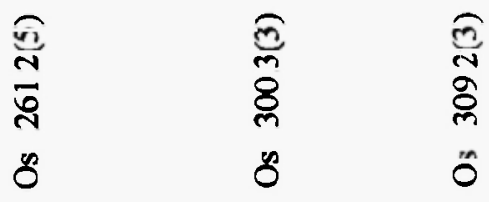

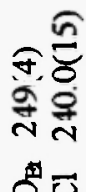

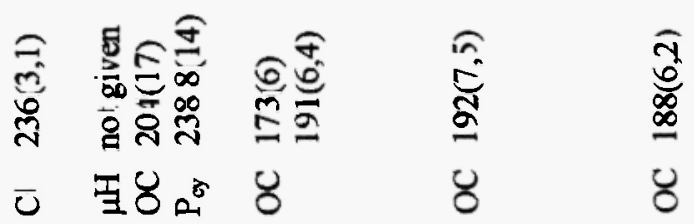

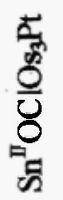

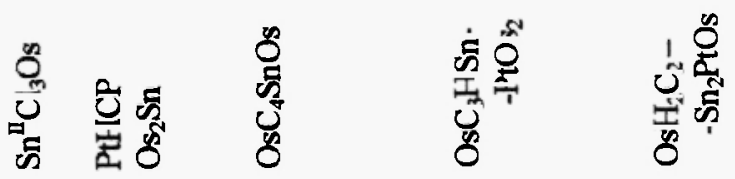

กิ్

$\infty \infty \infty$

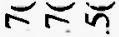

$\infty$ 舟芦

$\stackrel{\infty}{\simeq}$ 유

घ

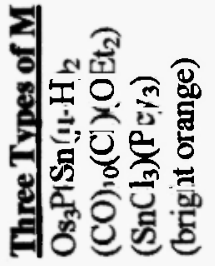


$\stackrel{\infty}{\pi}$

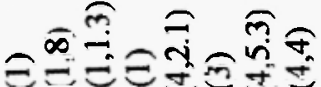

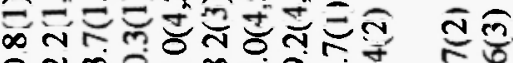

8워용요

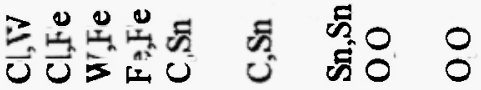

Еิ

究哭

노

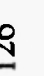

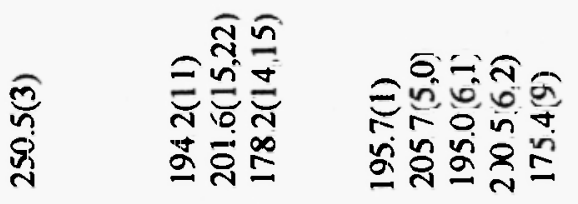
उ 8 \& $९ 98$

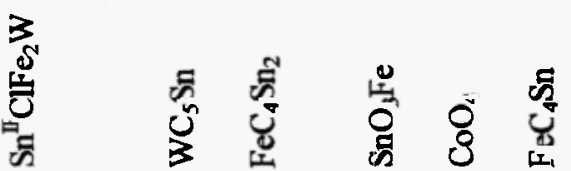

กิฐสิ

든

क동

ลิ

ปั ลิธ

오 8 พี

ลิธูำ

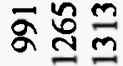

ลำำ

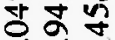

$\infty$ is

ชิํำ

$\stackrel{\infty}{0}$ 品

b-

$\nabla_{1} \bar{\Omega}$
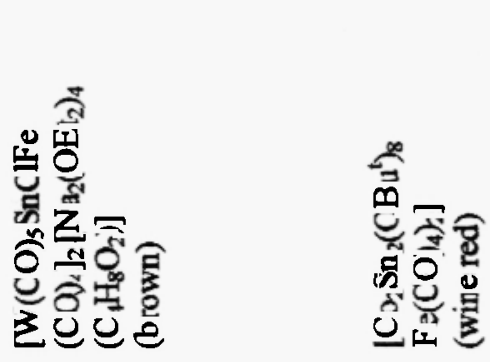
tetrahedral environment. with some in distorted tetrahedral or pseudo-octahedral situations. The most common liagnds for $\mathrm{Sn}$ are the methyl group or a $\mathrm{CI}$ atom. There are three hexameric clusters which contain two crystallographically independent molecules, differing mostly by degree of distortion $[96,208,209]$, and which belong to the general class of distortion isomers [113]. The metal-metal distances in the hexamers are listed in Table 4a. The Sn-Fe bond distance of 248.0(1) [226] is the shortest in the series of hexameric heterotin clusters.

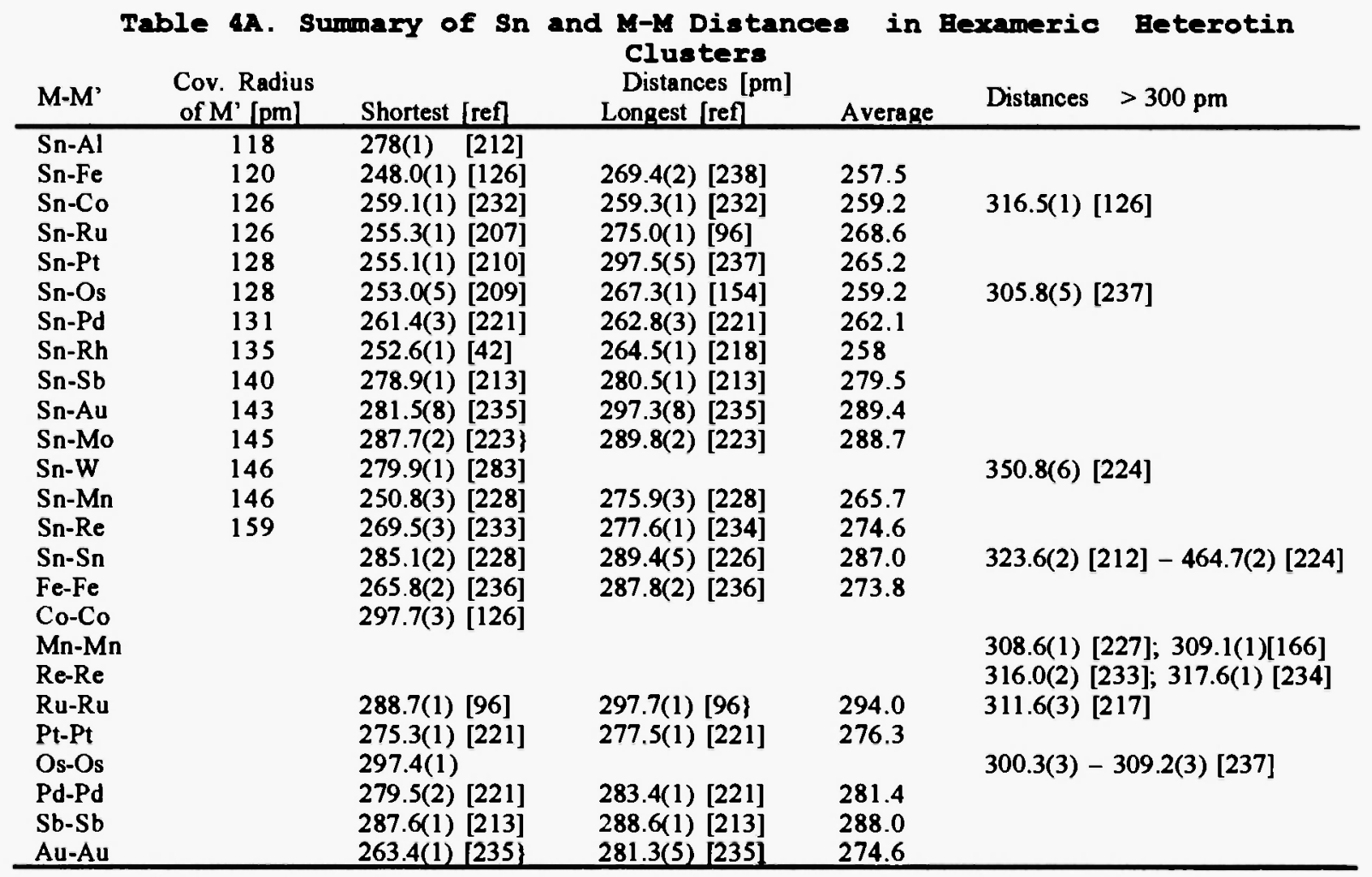

\section{HEPTAMERIC HETEROTIN COMPOUNDS}

There are thirteen heptameric heterotin clusters summarised in Table 5 and referenced in order of devreasing number of tin atoms, without sub-headings. Only transition heterometal atoms are found in this series.

The structure of an orange-yellow $\mathrm{Sn}_{6} \mathrm{Fe}$ cluster is shown iFigure 12. The central part of the molecule contains a ball framework of $\mathrm{SnO}_{6}$. The $\mathrm{Fe}(\mathrm{CO})_{4}$ moiety is directly bound to a $\mathrm{Sn}$ atom with the $\mathrm{Sn}-\mathrm{Fe}$ bond distance of $247.2 \mathrm{pm}$.

An orange $\mathrm{Sn}_{2} \mathrm{Mn} 4$ cluster [240] has a cubane-like $\mathrm{Sn}_{3} \mathrm{~S}_{4} \mathrm{Mn}$ cage. Each $\mathrm{Sn}$ (II) atom is bonded to a $\mathrm{Mncp}(\mathrm{CO})_{2}$ moiety and completes a quasi-tetrahedral environment about each $\mathrm{Sn}$ (II) atom ( $\mathrm{SnS}_{3} \mathrm{Mn}$ ) with the $\mathrm{Sn}-\mathrm{Mn}$ bond distance of $245.5(1) \mathrm{pm}$. A pale brown $\mathrm{Sn}_{3} \mathrm{Fe}_{4}$ cluster [230] contains a tricyclic $\mathrm{Sn}_{3} \mathrm{~S}_{4} \mathrm{Fe}$ cage. Two rings are five membered $\mathrm{Sn}_{2} \mathrm{~S}_{2} \mathrm{Fe}$ and the other is four membered $\mathrm{Sn}_{2} \mathrm{~S}_{2}$, requiring that two $\mathrm{Sn}$ atoms and one $\mathrm{S}$ atom belong to all three rings. Each $\mathrm{Sn}$ atom is also bonded to an $\mathrm{Fe}\left(\mathrm{cp}(\mathrm{CO})_{2}\right.$ moiety. The ring $\mathrm{Fe}$ is also coordinated to a terminal $\mathrm{CO}$ group and a cyclopentadienyl ligand. The Sn-Fe bond distances range from 250.3(2) to 257.0(2) pm (average 252.3 pm). Each $\mathrm{Sn}$ atom is quasi-tetrahedral, two with the chromophore $\mathrm{SnS}_{3} \mathrm{Fe}$ and one with $\mathrm{SnS}_{2} \mathrm{Fe}_{2}$. Dark green $\mathrm{Me}_{4} \mathrm{Sn}_{3} \mathrm{Fe}_{4}(\mathrm{CO})_{16}$ [241] contains almost exactly planar $\mathrm{Sn}_{2} \mathrm{Fe}_{2}$ rings, causing the compression of the average $\mathrm{Sn}-\mathrm{Fe}-\mathrm{Sn}$ angle to $77.9^{\circ}$. The $\mathrm{Fe}-\mathrm{Sn}-\mathrm{Fe}$ angles within the ring are at $105.2^{\circ}$ at the terminal $\mathrm{Sn}$ atom and $98.8^{\circ}$ at the central $\mathrm{Sn}$ atom. Each $\mathrm{Sn}$ atom is tetrahedrally coordinated, the central tin bonded to four $\mathrm{Fe}(\mathrm{CO}) 4$ groups (average $\mathrm{Sn}-\mathrm{Fe}=274.7 \mathrm{pm}$ ), and the terminal tin to two $\mathrm{Fe}(\mathrm{CO})_{4}$ groups (average $\mathrm{Sn}-\mathrm{Fe}=262.5 \mathrm{pm}$ ) and two methyl groups.

The molecule of a green cluster [242] can be described as a hexametallic Ir4Sn2 planar "raft" surmounted by a $\mu_{4}-\mathrm{RSnO}_{3}$ "sail". The geometry about the capping tin of the sail is that of a highly distorted trigonal bipyramid $\left(\mathrm{SnO}_{3} \mathrm{CIr}\right.$ ) with $\mathrm{Sn}$-Ir bond distance of $263.7(6) \mathrm{pm}$. The remaining $\mathrm{Sn}$ atoms are quasi-tetrahedral $\left(\mathrm{SnC}_{2} \mathrm{Ir}_{2}\right)$ with Sn-Ir bond distances of 267.6(6) and 268.0(6) pm.

The structure of a black $\mathrm{Sn}_{2} \mathrm{Fe}$ s cluster [243] consists of an equatorial $\mathrm{Fe}_{3}(\mathrm{CO})_{9}$ triangular cluster capped 


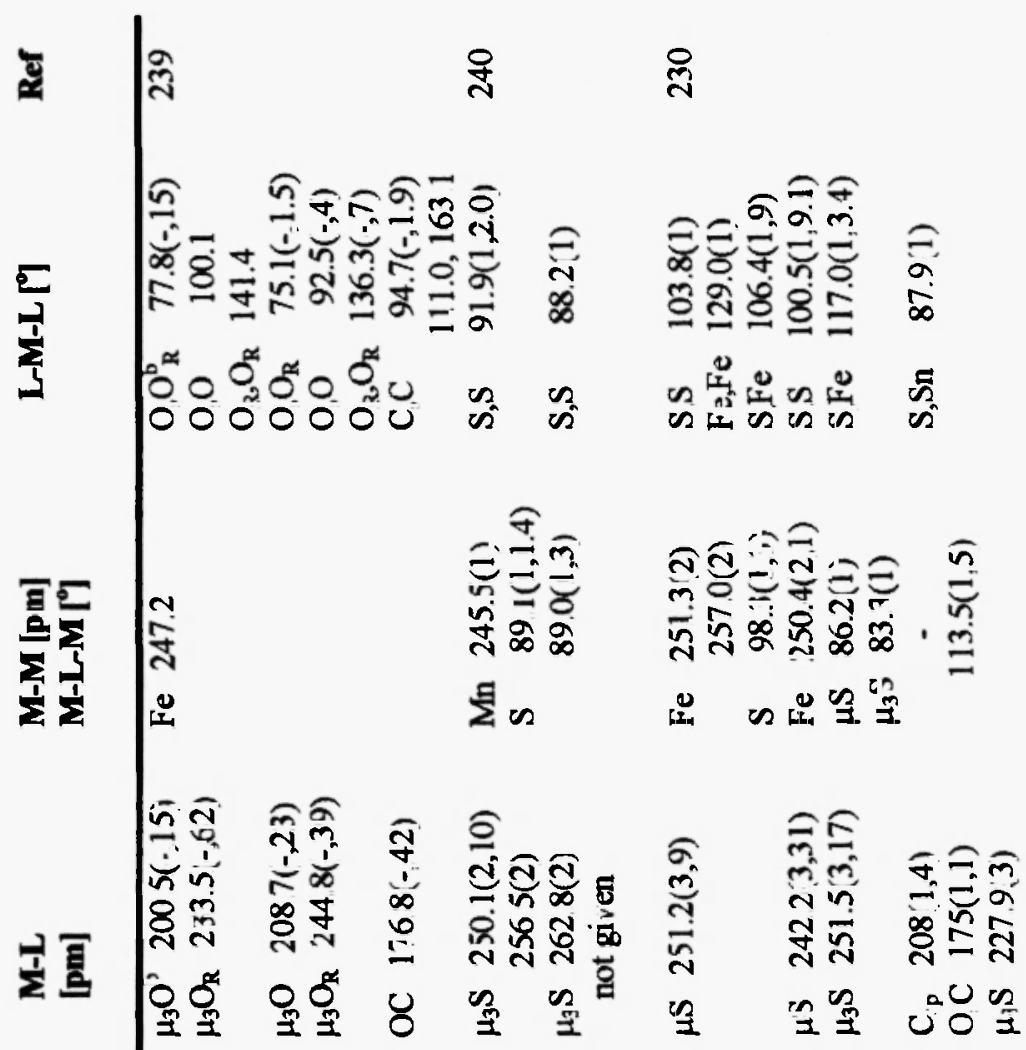

"

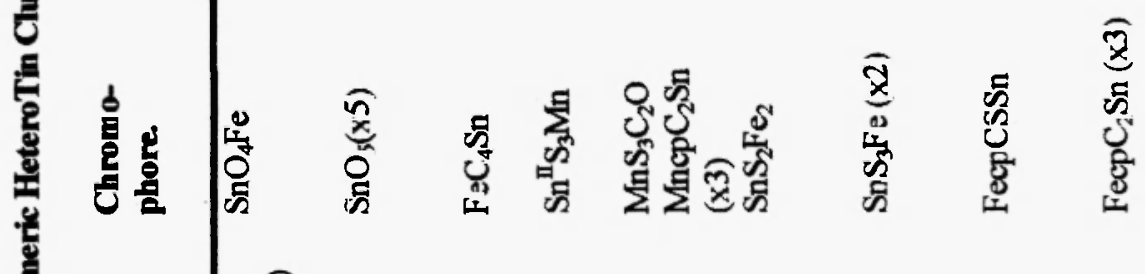

\begin{tabular}{|c|c|c|c|}
\hline$\sum_{\theta} \sum_{0}$ & 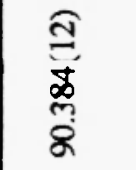 & 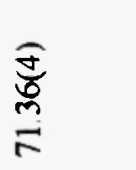 & 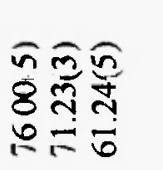 \\
\hline 豆星寔 & 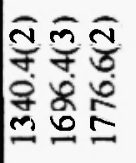 & $\begin{array}{l}\text { Fิ } \\
\alpha \\
0 \\
8 \\
=\end{array}$ & 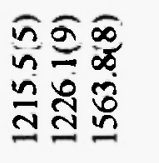 \\
\hline 迹N & ह $\stackrel{\text { E }}{\mathrm{E}}+$ & $E^{\prime} \ddot{\simeq} N$ & 淂五 \\
\hline 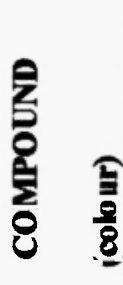 & 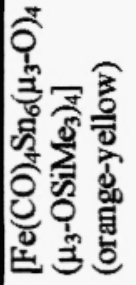 & 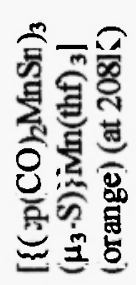 & 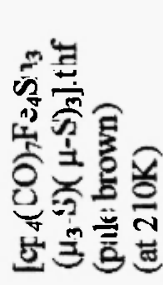 \\
\hline
\end{tabular}


$\underset{\sim}{\bar{T}}$

$\approx \stackrel{\text { กิ }}{-\infty}$

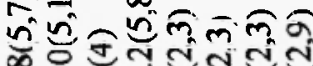

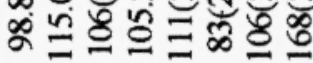

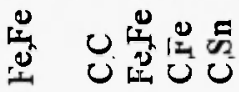

อิธอัดิ

m

究命事通

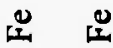

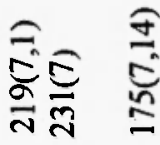

ป

क

$\underset{\substack{n \\ \infty}}{\infty}$

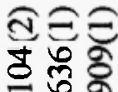

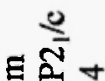

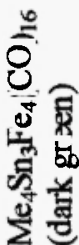

$\stackrel{\text { ก }}{2}$

$\stackrel{m}{\sim}$

$\stackrel{ \pm}{\sim}$

$\stackrel{n}{\sim}$

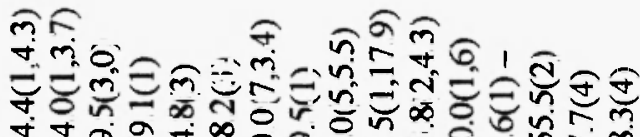

छ

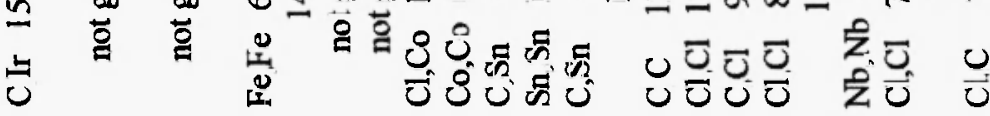

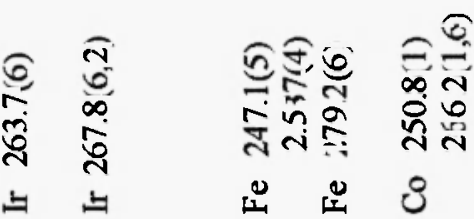

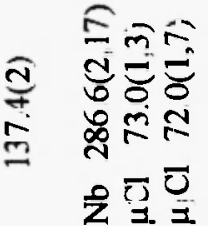

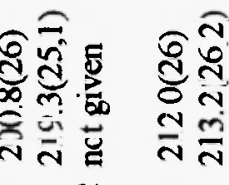

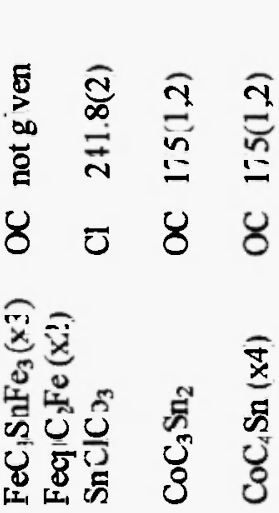

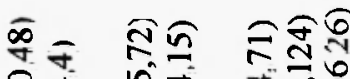

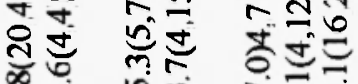

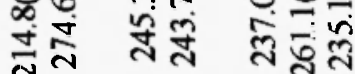

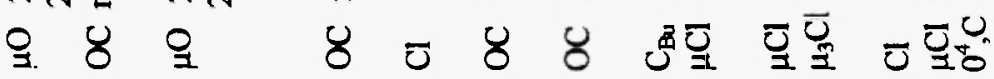

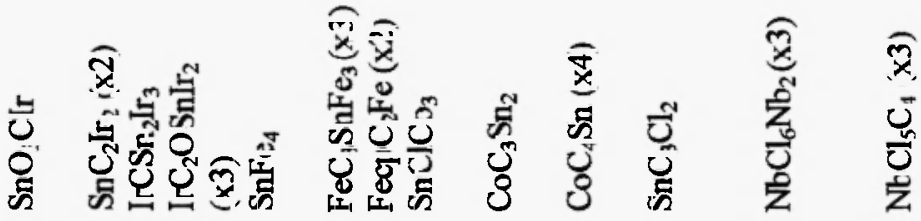

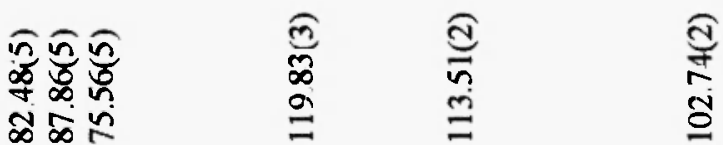

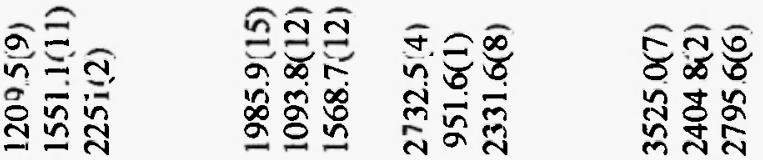

•ロล

E $\frac{\tilde{n}^{2}}{\text { a }}+$

$\varepsilon \stackrel{\circlearrowright}{\widetilde{U}} \infty$

'I
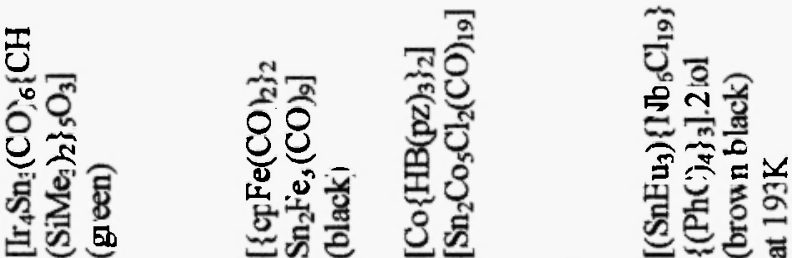
$\stackrel{\circ}{\text { 윤 }}$

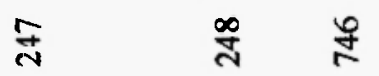

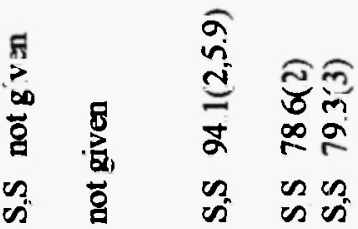

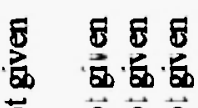

그를

旅穴

흥ㅎㅁ욤

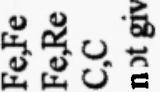

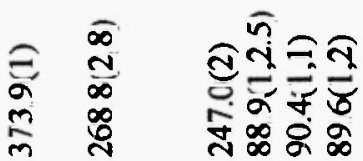

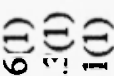

ब声

先瓷

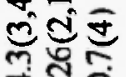

芛

$\sum \sum \sum$

เ

ఖ జ

วิ ชิศ สิ๋

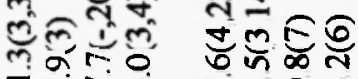

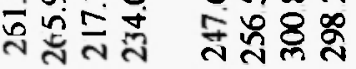

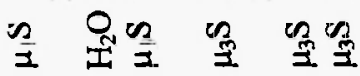

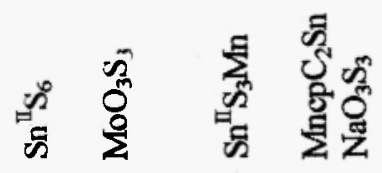

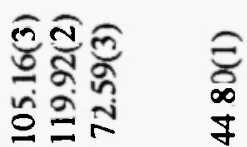

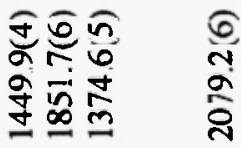

घี-

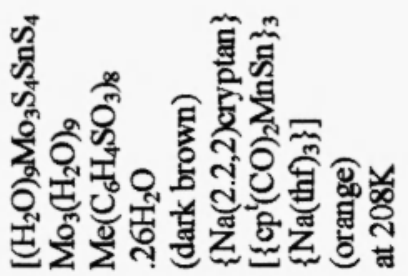

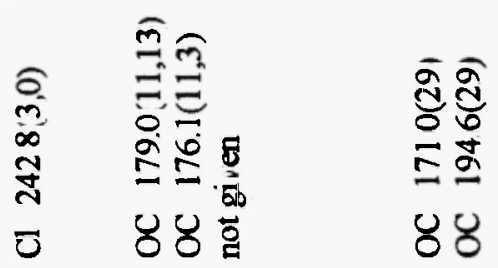
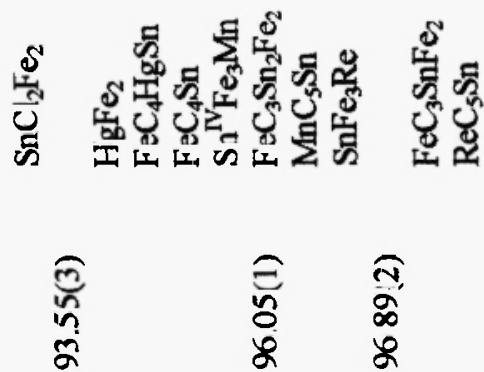

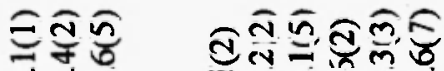

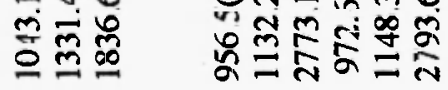

a $\frac{A^{2}}{E} \quad$ a

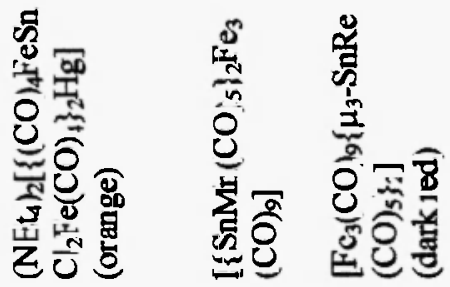



$\approx$

(2)

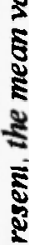

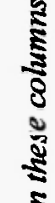

年 5

$-\frac{2}{2}$

$5 \%$

के

ช

ปั

ป气

है

(⿻)

จ. के

258

के

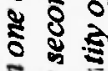

ปิ

อิ่

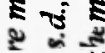

\& 80

$\pm+$

0 . 
on both sides by $\mathrm{SnFe}(\mathrm{CO})_{2} \mathrm{cp}$ fragments. All carbonyl groups in the molecule are terminally bonded. Three of them are approximately in the equatorial plane, which roughly defines a non-crystallographic mirror plane for the molecule. The average distances in the core of the molecule are: Sn-Fe(terminal) 247.1(5) pm; Sn-Fe(equatorial) 253.7(4) pm; Fe-Fe 279.2(6) pm.

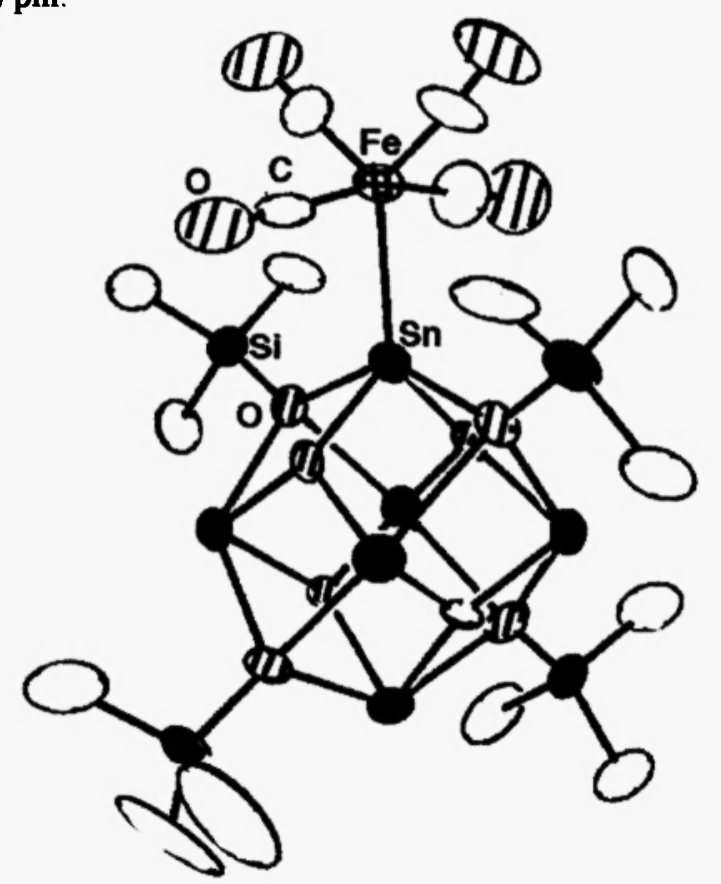

Fig. 12. Structure of [Fe(CO)4Sno(4(OSiMes)4] [239]

The structure of $\left[\mathrm{Co}\left\{\mathrm{HB}(\mathrm{pz})_{3}\right\}_{2}\right]^{4}\left[\mathrm{Sn}_{3} \mathrm{Co}_{5} \mathrm{Cl}_{2}(\mathrm{CO})_{19}\right]$ [244] consists of discrete cations and anions. the latter consists of two $\mathrm{Sn}(\mathrm{Cl})\left\{\mathrm{Co}(\mathrm{CO})_{4}\right\}_{2}$ groups bonded trans to each other about the planar $\mathrm{Co}(\mathrm{CO})_{3}$ fragment. Within these groups the $\mathrm{Co}(\mathrm{CO})_{4}$ moieties have a trigonal bipyramidal geometry $\left(\mathrm{CoC}_{3} \mathrm{Sn} 2\right)$ with average $\mathrm{Sn}$ - $\mathrm{Co}$ bond distance of $266.2(1) \mathrm{pm}$. Both tin atoms are tetrahedrally coordinated (SnClCo3) with the Sn-Co(central) bond of 250.8(1) pm, which is about $15.4 \mathrm{pm}$ shorter than the remaining two Sn-Co bond distances (average 266.2(1) pm).

A brown-black $\mathrm{SnNb}_{6}$ cluster [245] contains a central $\mathrm{Nb}_{3}$ cluster plus three outer $\mathrm{Nb}$ atoms bonded to $\eta^{4}$-tetraphenylcyclobutadiene ligands. The Bu3 Sn unit has an effectively trigonal-planar structure with $\mathrm{CI}$ atoms occupying axial positions to complette a trigonal-bipyramid. The entity consists of a central triangular $\mathrm{Nb}_{3}\left(\mu_{3}-\right.$ $\mathrm{Cl})(\mu-\mathrm{Cl})_{3}$ cluster with $\mathrm{Cl}_{3} \mathrm{NbCl}_{2}\left(\eta-\mathrm{C}_{4} \mathrm{Ph}\right)$ units. A dark brown SnMos cluster [246] contains a heterometal double cubane type $\mathrm{Mo}_{3} \mathrm{~S}_{4} \mathrm{SnS}_{4} \mathrm{MO}_{3}$ core with the mean Mo-Mo distance of $268.8(2) \mathrm{pm}$, with a six coordinate $\mathrm{Sn}(\mathrm{II})$ atom ( $\left.\mathrm{SnS}_{6}\right)$.

The structure of an orange $\mathrm{Sn}_{3} \mathrm{Mn} 3 \mathrm{Na}$ cluster [240] is similar to that of the $\mathrm{Sn}_{3} \mathrm{Mn}_{4}$ cluster [240] in which the Mn atom is substituted by Na to give a $\mathrm{Sn}_{3} \mathrm{~S}_{4} \mathrm{Na}$ core. Each $\mathrm{Sn}$ (II) atom is also bonded to a $\mathrm{Mn}(\mathrm{CO})_{2} \mathrm{cp}$ moiety, giving a quasi-tetrahedral arrangement about each $\mathrm{Sn}(\mathrm{II})$ atom ( $\mathrm{SnS} 3 \mathrm{Mn}$ ) with Sn-Mn bond distance of 247.0(2) pm. An orange $\mathrm{Sn}_{2} \mathrm{Fe}_{4} \mathrm{Hg}$ cluster [247] contains the unbridged FeSnFeHgFeSnFe skeleton with metal-metal bonds of value: Hg-Fe 257.1(1) pm; Sn-Fe(outer) 249.6(1) pm and Sn-Fe(inner) 259.3(1) pm.

Table 5A. Summary of Sn-M and M-M Distances in Beptameric Beterotin
Clusters


The data in Table 5 show tin to be predominantly tetrahedral in these derivatives, with some examples of trigonal-bipyramidal and octahedral coordination with varying degrees of distortion. The most common heterometal atom is iron, and there are only four donor ligand atoms, $\mathrm{O}, \mathrm{S}, \mathrm{Cl}$ and $\mathrm{C}$. The metal-metal bond distances are summarised in Table 5A, where it can be seen that the shortest is Sn-Mn at 245.5(1) pm.

\section{OCTAMERIC HETEROTIN COMPOUNDS}

The data for twelve octameric heterotin compounds are given in Table 6. The structures are listed and discussed in order of decreasing number of tin atoms. The heterometal atoms include both transition and non-transition metals.

The highest number of tin atoms is exhibited in an orange red $\mathrm{Sn}_{6} \mathrm{Fe}_{2}$ cluster [239] which has a structure similar to that of a previous $\mathrm{Sn}_{6} \mathrm{Fe}$ cluster shown in Figure 12 [239]. In the octamer an additional $\mathrm{Fe}(\mathrm{CO})_{4}$ moiety is bonded to the $\mathrm{Sn}(6)$ atom with an $\mathrm{Sn}-\mathrm{Fe}$ bond distance of $246.3(1) \mathrm{pm}$, shorter by $0.9 \mathrm{pm}$ than that found in the heptamer. There are two sets of $\mathrm{Sn}$ atoms, one with distorted trigonal-bipyramidal geometry $\left(\mathrm{SnO}_{4} \mathrm{Fe}\right)$ and the other with distorted tetrahedral geometry $\mathrm{SnO}_{4}$.

A red Sn`Li4 cluster [113a] contains a fourteen membered $\mathrm{Sn}_{4} \mathrm{P}_{6} \mathrm{Li}_{4}$ core. The molecule consists of an association between the $\left[\mathrm{Sn}_{2}(\mathrm{Pcy})_{2}\right]$ anion and four $\left[\mathrm{Li}(\mathrm{thf})_{4}\right]^{+}$cations. The anion can be viewed as constructed of two puckered $\mathrm{Sn}_{2}$ (Pcy)2 dimer rings linked by two dimer bridging Pcy groups. The four $\mathrm{Li}^{+}$cations are bonded to the $P$ centres of the $S_{2}(\mu-P c y)_{2}$ dimer units and to the $P$ centres of the dimer in the equatorial position of the anion. The Sn(II) atom is tricoordinate $\left(\mathrm{SnP}_{3}\right)$. A colourless $\mathrm{Sn}_{4} \mathrm{Al}_{4}$ cluster [249] has a central $\mathrm{O}_{2} \mathrm{Sn}_{4} \mathrm{Cl}_{2}$ core of approximately $\mathrm{D}_{2 \mathrm{~h}}$ symmetry, with four coordinate $\mu_{4}-\mathrm{O}$ and $\mu_{4}-\mathrm{Cl}$ atoms. The (Pr'O)s $\mathrm{Al}_{2}$ dimers are connected with the central core by $\mu_{4}-\mathrm{O}$ and $\mu-\mathrm{OPr}^{i}$ bridged groups. Each metal atom has a distorted trigonal-bipyramidal environment ( $\mathrm{SnO}_{3} \mathrm{Cl}_{2}$ and $\mathrm{AlO}_{4} \mathrm{Cl}$ ). Another colourless $\mathrm{Sn}_{4} \mathrm{Al}_{4}$ cluster [250] is shown in Figure 13. It is composed of dimeric units $\left[\left(\eta^{6}-\mathrm{C}_{0} \mathrm{Me}_{6}\right) \mathrm{SnCl}\left(\mathrm{AlCl}_{4}\right)\right]_{2}$ containing crystallographically non-equivalent monomers. These dimers are cross-linked to form centrosymmetrical tetramers. The molecular unit of the white $\mathrm{Sn}_{4} \mathrm{Cd}_{4}$ cluster [251] includes a planar eight membered ring of four $\mathrm{Cd}$ atoms linked in pairs by single $\mathrm{O}$ atoms of two acetate anions. Each of these is also chelated to one of the $\mathrm{Cd}$ atoms being bridged. The ring is completed by two oxo-groups, each linking a pair of $\mathrm{Cd}$ atoms on opposite sides of the ring. Pairs of $\mathrm{Sn}$ atoms are also bonded to the oxo-groups, making them four coordinate.

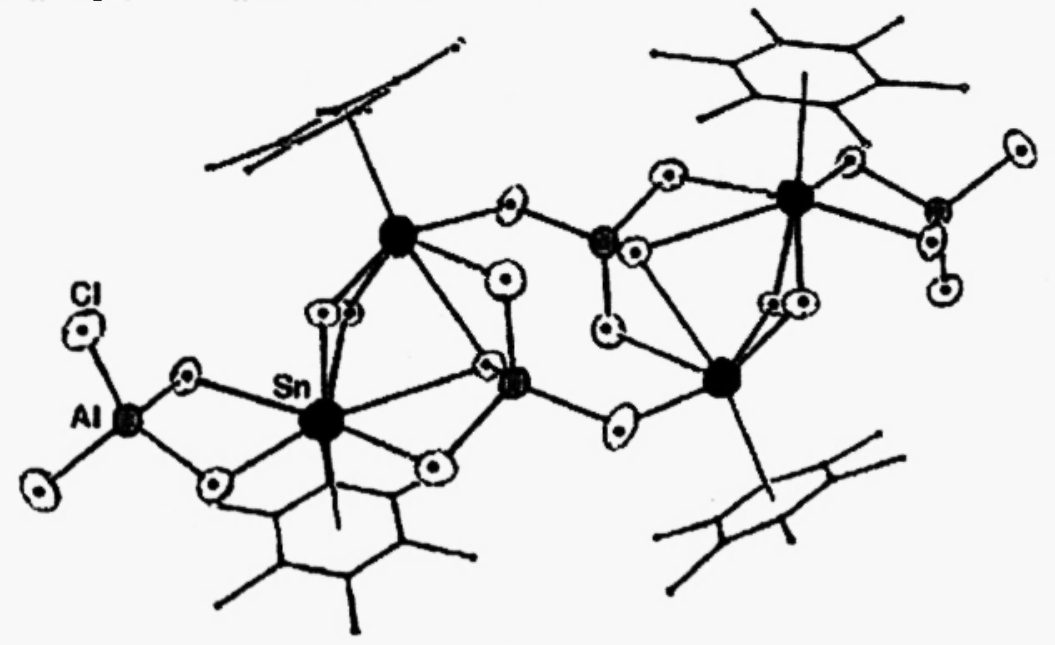

Fig 13. Structure of $\left[\left(\mathrm{C}_{6} \mathrm{Me}_{6}\right) \mathrm{SnCl}\left(\mathrm{AlCl}_{4}\right)\right]_{4}[250]$

The structure of a deep red $\mathrm{Sn}_{4} \mathrm{MO}_{4}$ cluster [223] consists of an adamantane-like $\mathrm{Sn}_{4} \mathrm{Te}_{6}$ framework. Each $\mathrm{Sn}$ atom is bonded to a $\mathrm{Mo}(\mathrm{CO})_{2} \mathrm{cp}$ unit with average Sn-Mo bond distance of 280.2(3) pm. Each Sn atom has a quasi-tetrahedral environment (SnTesMo). A Snd $\mathrm{W}_{4}$ cluster [252] contains two structurally similar but symmetry independent molecules in a unit cell. Each has a crystallographically imposed centre of symmetry located in the middle of the sixteen membered ring, which is formally a [-O-Wcp (O)-O-SnMe3-]4 tetramer. The interplanar angle between the $\mathrm{Sn}_{4}$ and $\mathrm{W}_{4}$ planes in one molecule is $11^{\circ}$ and in the other is $14^{\circ}$. Each $\mathrm{Sn}$ atom has a trigonal bipyramidal arrangement $\left(\mathrm{SnC}_{3} \mathrm{O}_{2}\right)$, and each $\mathrm{W}$ atom has a typical three legged piano-stool geometry. There are two orange [230] and one yellow [253] $\mathrm{Sn}_{4} \mathrm{Fe}_{4}$ clusters, all isostructural with an adamantanelike cage $\mathrm{Sn}_{4} \mathrm{X}_{6}\left(\mathrm{X}=\mathrm{S}[253]\right.$ or Se [230]). Each of four Fecp $(\mathrm{CO})_{2}$ moieties is coordinated to the $\mathrm{Sn}$ atom giving a quasi-tetrahedral environment ( $\mathrm{SnX} \mathrm{X}_{3} \mathrm{Fe}$ ). The mean $\mathrm{Sn}-\mathrm{Fe}$ bond distances are $250.4 \mathrm{pm}$ [253] and 252.6 pm [230]. 


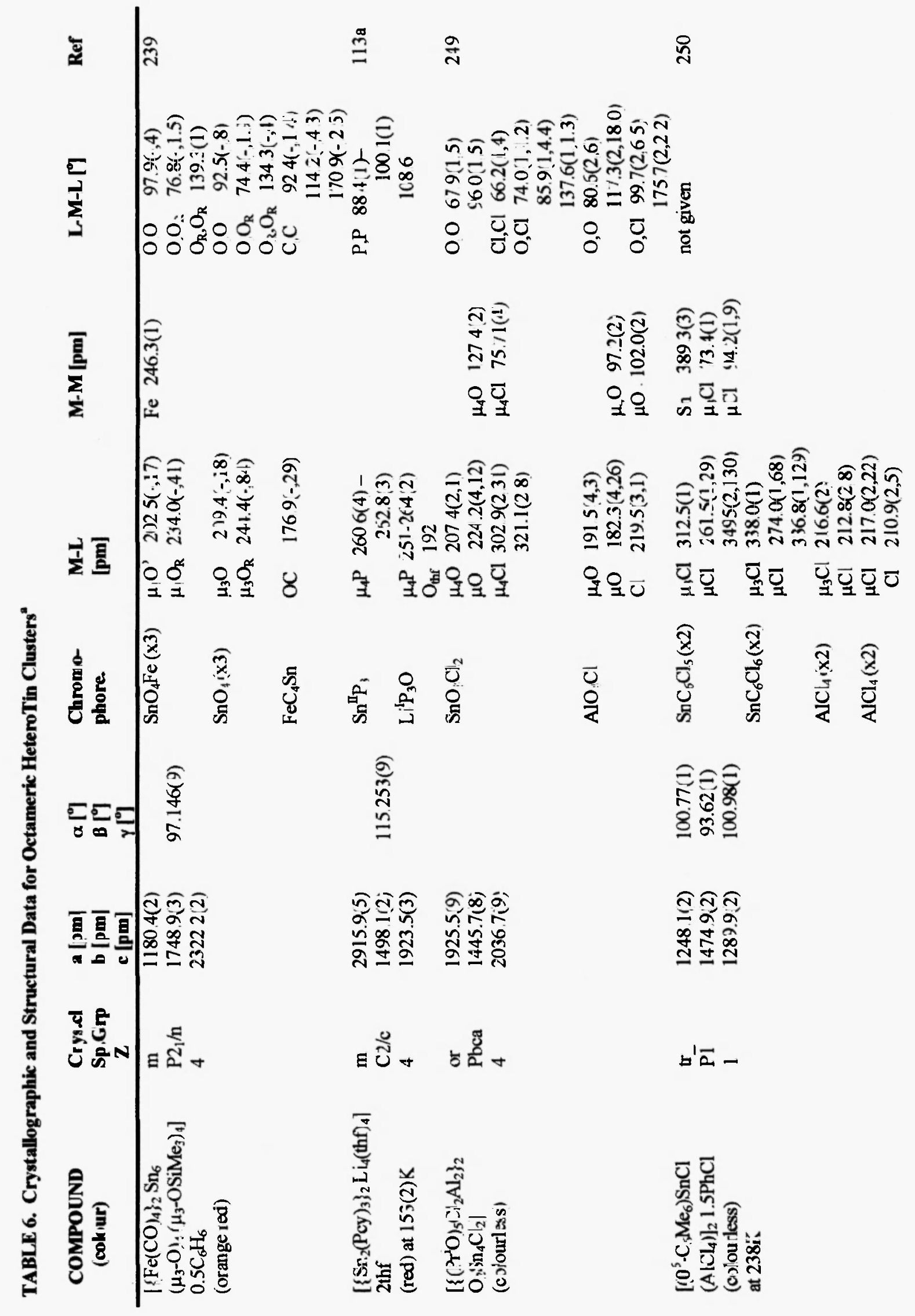


$\overline{\text { ป }}$

สิก กู

$\approx$ กำ

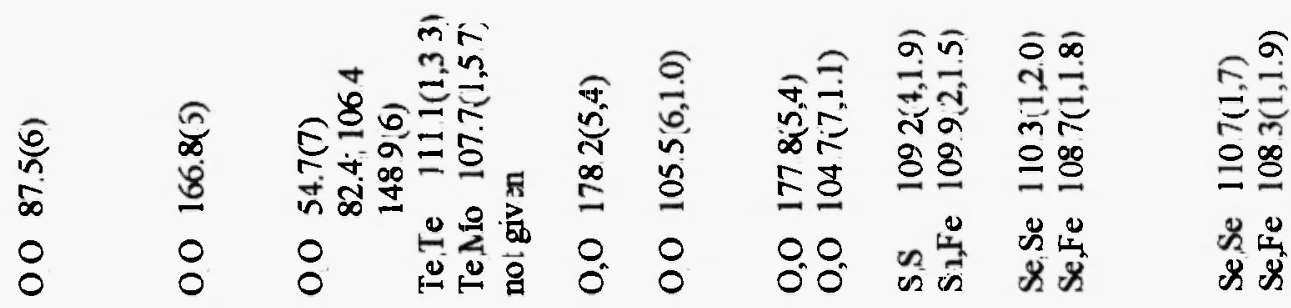

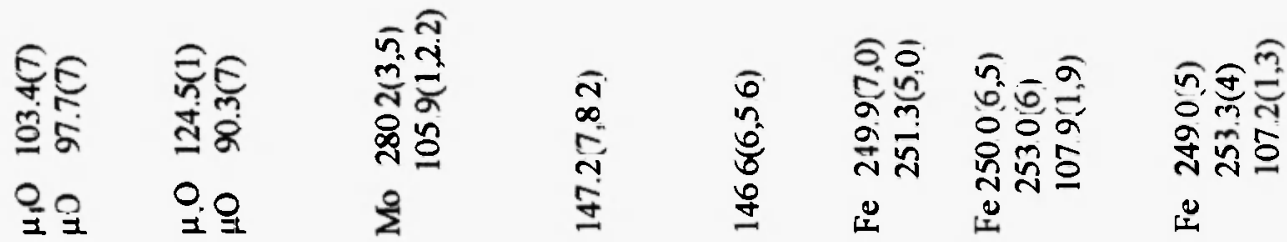

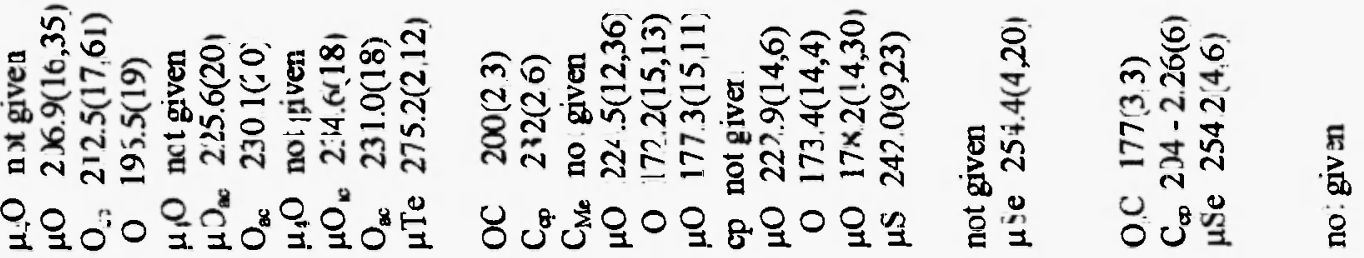

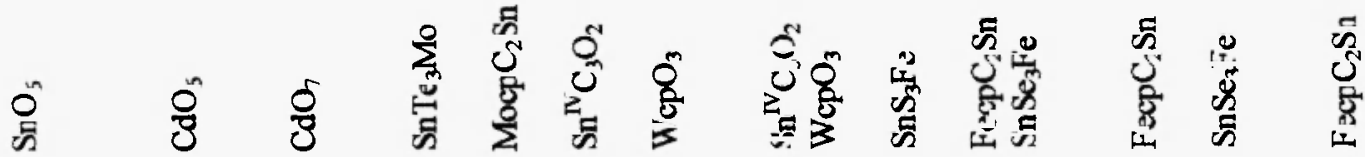

$\frac{\widehat{\theta}}{\frac{8}{6}}$

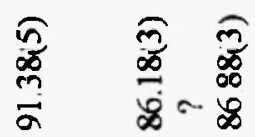

$\underset{\frac{d}{2}}{\frac{2}{a}}$

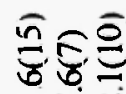

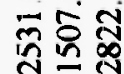

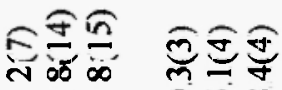

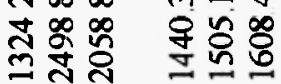

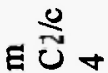

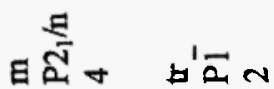
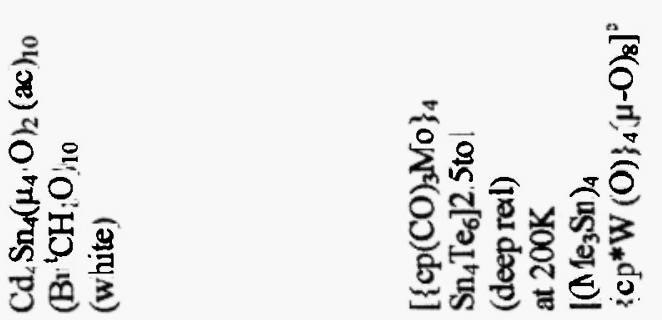

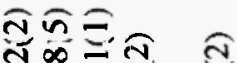

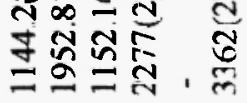

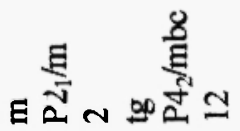

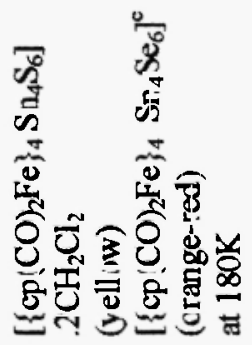




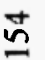

in $\bar{\sigma} \times$

$=0$

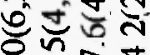

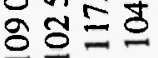

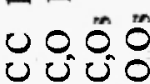

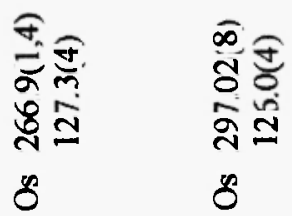

啇交

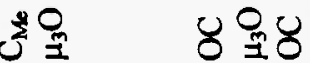

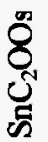

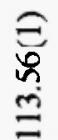

ํํำิ

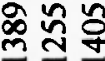

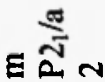

劳

तै

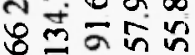

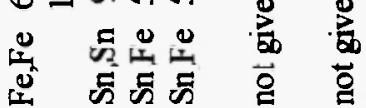

สิสิ

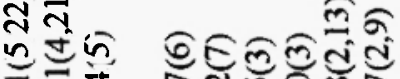

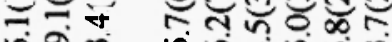

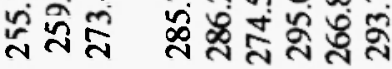

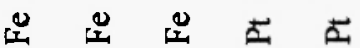

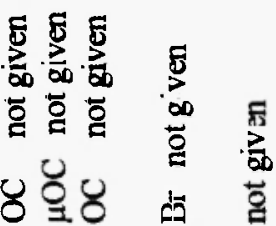

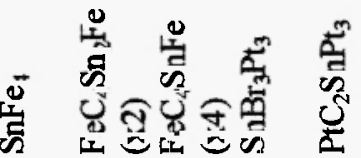

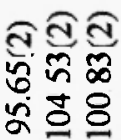

昰哭

๙ กิ

ָูป

$\operatorname{an}$

घ $\stackrel{\text { ลे }}{2}$

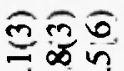

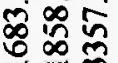

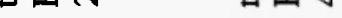
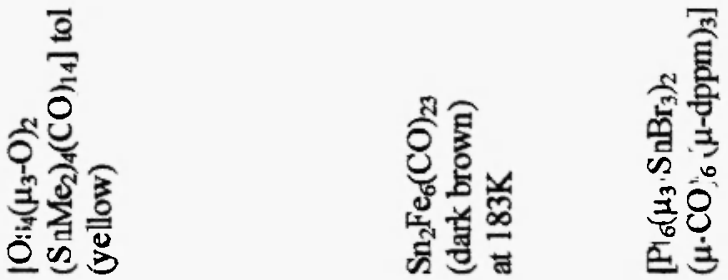
A yellow $\mathrm{Sn}_{4} \mathrm{Oss}_{4}$ cluster [154] has a crystallographic 2-fold axis that passes through the centre of an $\mathrm{Os}_{2} \mathrm{Sn}_{2} \mathrm{O}_{2}$ ring. Four membered, essentially planar, $\mathrm{Os2}_{2} \mathrm{SnO}$ rings share $\mathrm{OsO}_{5} \mathrm{O}$ edges with the central ring. The dihedral angle between the planes is $1.5^{\circ}$. The central ring has a boat conformation, with dihedral angle of $46.4^{\circ}$. Each tin atom has a quasi-tetrahedral environment ( $\mathrm{SnC}_{2} \mathrm{OO}$ s) with average $\mathrm{Sn}-\mathrm{Os}$ bond distance of 266.9(1) pm.

A dark brown $\mathrm{Sn}_{2} \mathrm{Fe}_{6}(\mathrm{CO})_{23}$ cluster [254] contains four $\mathrm{SnFe}_{2}$ triangles, as shown in Figure 14. Two triangles with a common edge have a dihedral angle of $73.1(1)^{\circ}$ at one $\mathrm{Sn}$ atom and $83^{\circ}$ at the other. The butterfly angle between the two central triangles is $115.8(1)^{\circ}$. The Sn-Fe bond distance ranges from $252.9(5)$ to $261.2(4) \mathrm{pm}$ (average $256 \mathrm{pm}$ ), while the $\mathrm{Sn}-\mathrm{Sn}$ separation is essentailly non-bonding at $369 \mathrm{pm}$. A SnzPto cluster [255] is shown inFigure 15 . The molecule has approximately $D_{3 h}$ symmetry, and the metal core can be described as a trigonal prism of $\mathrm{Pt}$ atoms, with each triangual face capped by a SnBr3 group. Thus both the $\mathrm{Pt}_{3}$ triangles and the $\mathrm{SnBr}$ groups are eclipsed. The Pt-Pt bond length within each triangle ranges from $265.6(2)$ to 294.6(2) pm. The Pt-Sn distances are in the range 274.5(3) to 295.0(3) pm.

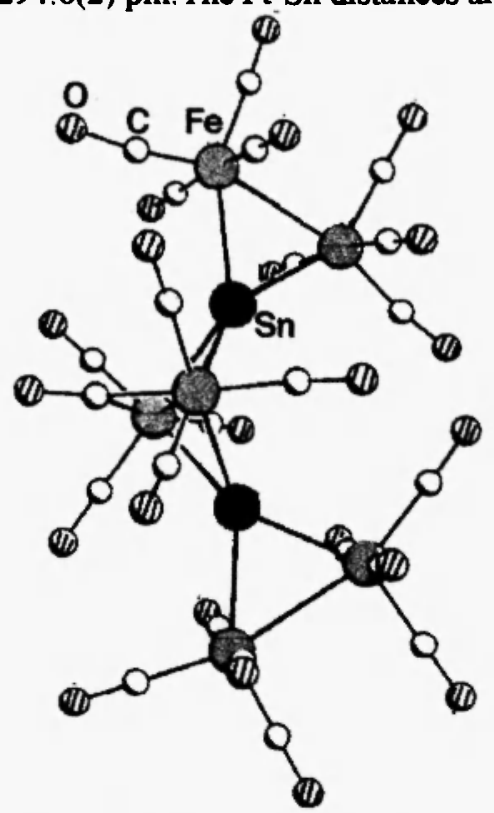

Fig.14. Structure of $\mathrm{Sn}_{2} \mathrm{Fe}_{6}(\mathrm{CO})_{23}[254]$

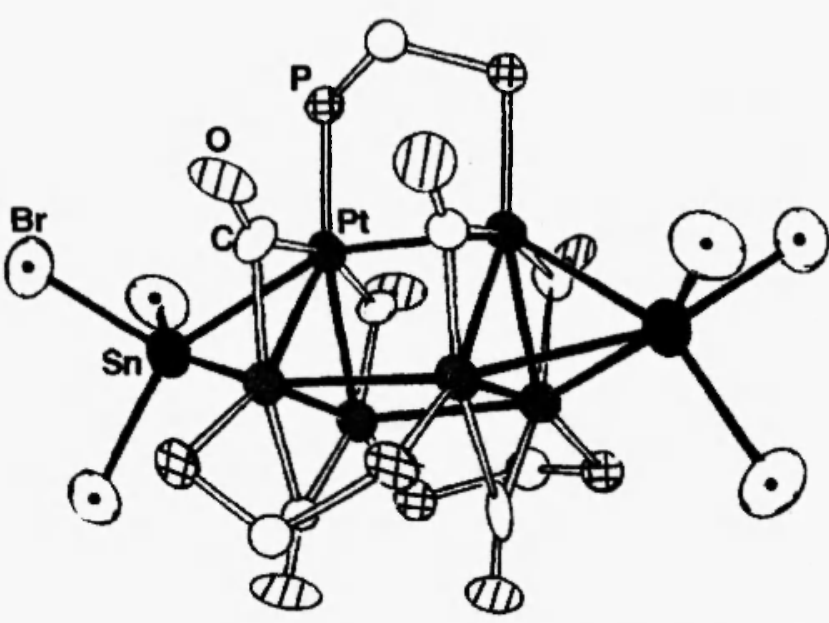

Fig.15. Structure of $\left[\mathrm{Pt}\left(\mu_{2}-\mathrm{SnBr}\right)_{2}(\mu-d p p m)_{3}\right]$ [255]

The data in Table 6 indicate the most favoured cluster is $\mathrm{Sn}_{4} \mathrm{M}_{4}$ (nine out of twelve). The most common donor atom is oxygen. Despite the few examples. tin was found in all its commonest coordination environments, tetrahedral, trigonal planar, trigonal bipvramidal and octahedral. Distorted tetrahedral was found to be the most common geometry. The metal-metal bon distances are summarised in Table 6A for the octameric derivatives. The shortest value was observed for Sn-Fe at 246.3(1) pm [239].

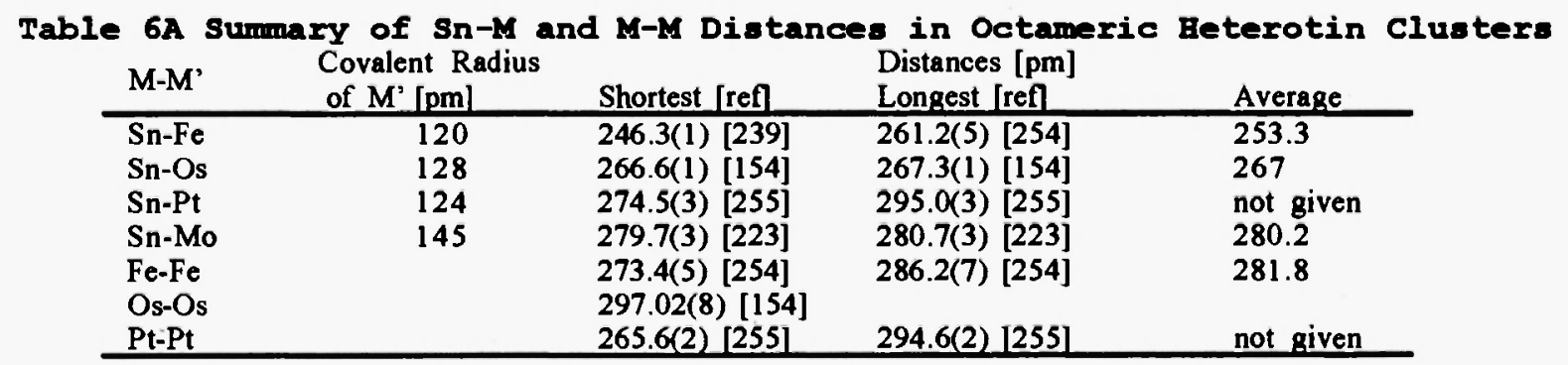

\section{OLIGOMERIC DERIVATIVES}

The data for almost twenty oligomeric derivatives are given in Table 7. The structures are listed in order of increasing nuclearity, and secondarily by decreasing number of tin atoms, without subheadings. All classes of heterometal atom are represented in this series.

Two anions, $\mathrm{TISn}_{8}$ and $^{-31 \mathrm{Sn}_{9}}{ }^{3}$ exhibit an unusual 50:50 occupational disorder in a single anionic site, with seven atoms coincident in both species of this red brown cluster [256]. The former has a tricapped trigonal- 
prismatic configuration, while the latter has a bicapped square-antiprismatic geometry. The average $\mathrm{Sn}-\mathrm{Tl}$ distance of $306.3 \mathrm{pm}$ in the former is about $55 \mathrm{pm}$ shorter than that found in the latter (311.8 pm). The Sn-Sn distance in the former ranges from 287.9(4) to $330.1(5) \mathrm{pm}$, and in the latter from 286.2(4) to $325.3(4) \mathrm{pm}$.

There are three coloured $\mathrm{Sns} \operatorname{Ln}(\mathrm{Ln}=\mathrm{Sm}$ or $\mathrm{Yb}$ ) isostructural clusters [257]. The Yb derivative exists in two isomeric forms, orthorhombic and tetragonal. The molecules of the clusters have a distorted octahedral structure in which the central $\mathrm{Ln}$ atom is bonded to four $\mathrm{O}$ atoms of (thf) molecules equatorially and two $\mathrm{Sn}$ (II) atoms of two $\mathrm{Sn}\left(\mathrm{SnMe}_{3}\right)_{3}$ moieties axially. The $\mathrm{Sn}-\mathrm{Ln}-\mathrm{Sn}$ bond angles are $178.8(1)^{\circ}(\mathrm{Ln}=\mathrm{Sm}), 178.5(1)^{\circ} \mathrm{Yb}$, orthorhombic) and $176.2(1)^{\circ}$ (Yb, tetragonal). The average Ln-Sn distances are 339.4(4) pm (Sm), 329.4(5) ${ }^{4}(\mathrm{Yb}$, orthorhombic) and $330.0(4)^{\circ}$ (Yb, tetragonal). Two Sn(II) atoms in each cluster contain the SnLnSns chromophore with average Sn-Sn distances of $277.8(6), 278.6(6)$ and $280.0(4) \mathrm{pm}$, respectively. The remaining six $\mathrm{Sn}(\mathrm{II})$ atoms in each of the respective clusters contain the $\mathrm{SnC}_{3} \mathrm{C}$ chromophore.

A red cluster $\left[2581\right.$ contains $\left[\mathrm{Au}_{3}\left(\mathrm{PPh}_{3}\right) \gamma\left(\mathrm{SnCl}_{3}\right)^{+}\right.$cations and $\left[\mathrm{SnCl}_{6}\right]^{*}$ anions. The structure of the cation is shown in Figure 16. The gold atoms in the structure are arranged in a centred ring, all bonded to a central $\mathrm{Au}$ atom. The central $\mathrm{Au}$ to peripheral $\mathrm{Au}$ atom distances lie in the range 263.3(2) to 267.9(2) pm. In contrast, the Au-Au bond lengths for the peripheral atoms lie in the range 284.7(2) to 296.5(3) pm. The $\mathrm{SnCl}_{3}$ moiety is coordinated exclusively to the central Au atom with Sn-Au distance of $262.5(3) \mathrm{pm}$.

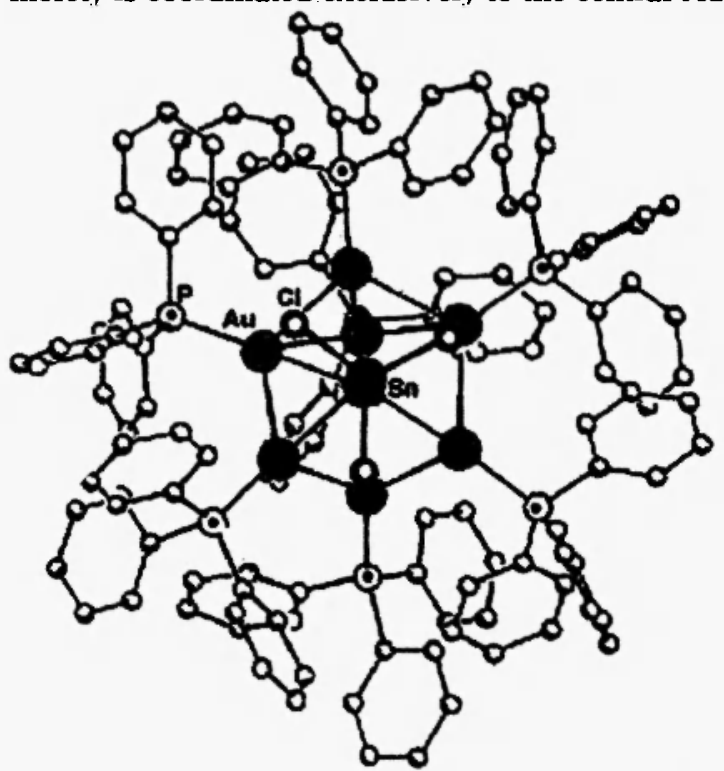

Fig. 16. (a) Structure of $\left[\mathrm{Auu}\left(\mathrm{PPh}_{3}\right)_{7}\left(\mathrm{SnCl}_{3}\right)_{2}\right]^{+}$.

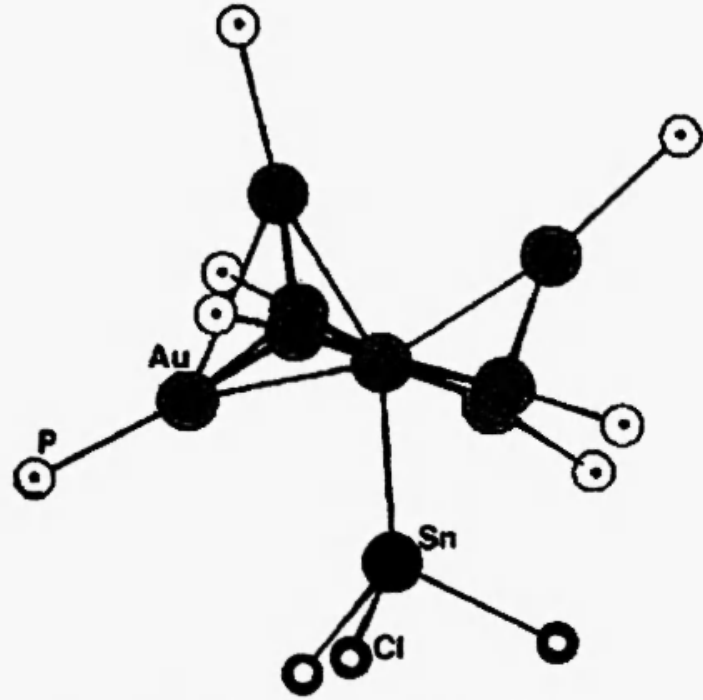

(b) Phenyl Rings omitted

[258]

A black cluster contains $\mathrm{K}[(\mathrm{cyp})]^{+}$cations and closo- $\left[\mathrm{Sn} \mathrm{Cr}(\mathrm{CO})_{3}\right]^{-4}$ anions [259]. There are two crystallographically independent anions in the unit cell that are structurally identical and reveals a virtually undistorted bicapped square antiprismatic shape. The formation of the cluster can be viewed as the insertion of a neutral $\mathrm{Cr}(\mathrm{CO})_{3}$ fragment into the open square face of a nido-Sn ${ }^{-4}$ ion. $\mathrm{The} \mathrm{Cr}(\mathrm{CO})_{3}$ fragment occupies a capping position in the bicapped square antiprism, with average $\mathrm{Sn}-\mathrm{Cr}$ bond length of 287.1(6) pm. The Sn-Sn distances within the square planes are 306(2) and 316(3) pm. The distances between the two square planes average at 299(2) pm.

An orange SnsMor cluster [262] has virtual $\mathrm{C}_{2 h}$ Symmetry with six metal atoms ( $\left.\mathrm{Sn}, \mathrm{Sn}, \mathrm{Mo}, \mathrm{Mo}, \mathrm{Sn}, \mathrm{Sn}\right)$ lying in a plane. The Mo-Mo distance of 220.1(2) pm indicates triple bonding. The average $\mathrm{Sn}-\mathrm{Mo}$ and $\mathrm{Sn}-\mathrm{Sn}$ bond distances are $277.8(2)$ and 277.492$)$ pm, respectively. All eight tin atoms are quasi-tetrahedral, two as $\mathrm{SnMoSn}_{3}$ and six as $\mathrm{SnC}_{3} \mathrm{Sn}$ chromophores.

A SnoNis cluster [261] contains a polycyclic $\mathrm{Sn}_{6} \mathrm{Se} 9$ cage enclosing a central $\mathrm{cpNi2}$ unit. Each of the remaining three $\mathrm{cp}(\mathrm{PPh}) \mathrm{Ni}$ moieties coordinate to a $\mathrm{Sn}$ atom, completing a distorted tetrahedral geometry about these three $\mathrm{Sn}$ atoms ( $\mathrm{SnSe} 3 \mathrm{Ni}$ ) with average $\mathrm{Sn}-\mathrm{Ni}$ bond distance of 243.5(3) pm. Another three $\mathrm{Sn}$ atoms have a trigonal bipyramidal arrangement ( $\mathrm{SnSe} 3 \mathrm{Ni2}$ ) with average $\mathrm{Sn}-\mathrm{Ni}$ bond length of $252.9(3) \mathrm{pm}$.

There are red and yellow $\mathrm{Sn}_{6} \mathrm{Cr}_{6}$ clusters $[262,2 \mathrm{~b}]$, and the structure of the former is shown iifigure 17. The six Sn atoms form an almost regular octahedron with $\mathrm{Sn}-\mathrm{Sn}$ distances from 293.0(1) to 296.0(1) pm. Each $\mathrm{Sn}$ atom is coordinated through its lone pair of electrons to one $\mathrm{Cr}(\mathrm{CO})$ s fragment. The $\mathrm{Sn}-\mathrm{Cr}$ bond vectors radiate outwards with $\mathrm{Sn}-\mathrm{Cr}$ bond lengths of 260.8(2) and 261.3(2) pm. The deviations from ideal $\mathrm{Sn}-\mathrm{Sn}-\mathrm{Cr}$ 
angle $\left(135^{\circ}\right)$ result from spatial requirements of the bulky $\mathrm{Cr}(\mathrm{CO})$ s groups (Figure 17), but overall are quite small. The yellow cluster $[22 \mathrm{~b}]$ contains $\left[\mathrm{Na}(12-\mathrm{crown}-4)_{2}\right]^{+}$cations and $\left[\left\{(\mathrm{CO})_{5} \mathrm{CrSn}\right\}_{6}\left(\mu_{3}-\mathrm{O}\right)_{4}\left(\mu_{3}-\mathrm{OH}\right)_{4}\right]^{-}$anions. The $\mathrm{Sn}_{6}\left(\mu_{3}-\mathrm{O}\right)_{4}\left(\mu_{3}-\mathrm{OH}\right)_{4}$ cage of the anion has a rhombododecahedral structure. Each $\mathrm{Sn}(\mathrm{II})$ atom acts as a ligand towards $\mathrm{Cr}(\mathrm{CO})$ s via the stereochemically active lone pair electrons, and each is five coordinate $\left(\mathrm{SnO}{ }_{4} \mathrm{Cr}\right)$ with average Sn-Cr bond distance of $253.8 \mathrm{pm}$. A similar structure is observed for an orange $\mathrm{Sn}_{6} \mathrm{Mn}_{6}$ cluster [240], with the $\mathrm{Cr}(\mathrm{CO})_{5}$ "ligands" replaced by $\mathrm{Cp}^{*}(\mathrm{CO})_{2} \mathrm{Mn}$ units, with average Sn-Mn bond lengths of $243.7 \mathrm{pm}$.

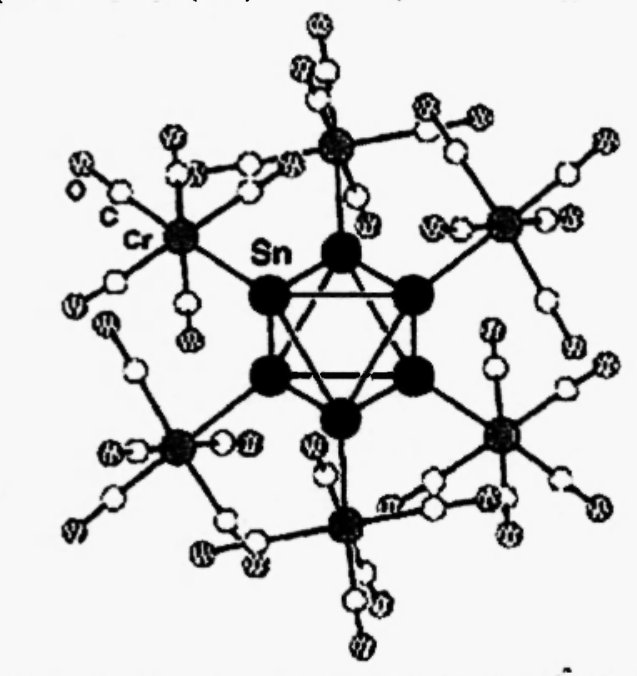

Fig.17. Structure of $\left[\operatorname{Sn}\left\{\left\{\operatorname{Cr}(\mathrm{CO})_{5}\right\} 6\right]=[262]\right.$

In SnAPtz cluster [263] the Pts metal core consists of three stacked edge-sharing tetrahedra of Pt atoms which generate four concave butterfly surfaces, each linked to one $\mathrm{SnCl}_{2}$ group. The whole $\mathrm{PtsSn}_{4}$ metal framework can be considered as the layering of two outer, symmetry related, Pt(I)-Pt(I) edges with two inner. symmetry related, $\mathrm{Pt}(2)-\mathrm{Pt}(2)-\mathrm{Sn}$ planar rhombuses. The chlorine atoms are essentially coplanar with the $\mathrm{Pt} \mathrm{Sn}_{2}$ rhombuses. Of the ten $\mathrm{CO}$ ligands eight are terminally bonded to the eight Pt atoms, and two double bridge the outer $\mathrm{Pt}(\mathrm{l})-\mathrm{Pt}(\mathrm{l})$ edges. The Pt tetrahedra are significantly elongated along the 4 axis, with average Pt-Pt bond lengths of 266.5(1) pm (intra-layer) and 292.5(1) pm (interlayer). The intra-layer Pt-Sn distance of 295.0(1) pm is longer than the average interlayer value of $260.4(1) \mathrm{pm}$.

There are two $\mathrm{Sn}_{2} \mathrm{~W}_{9}$ clusters [264] and the structure of one of them is shown iifigure 18. The anion has nominal $\mathrm{C}_{3 \mathrm{~h}}$ symmetry, with three $\mathrm{WO}_{6}$ octahedra of the $\beta$-Keggin anion replaced by three $\mathrm{PhSnO}_{3}$ groups.

Two red-brown Sn2Niıl clusters [256] contain the [PPlıMe] cation and $\left[\mathrm{Ni}_{11}\left(\mathrm{SnR}_{2}\right)(\mathrm{CO})_{18}\right]^{-4}$ anions $(\mathrm{R}$ $=\mathrm{Me}$ or $\mathrm{Bu}$ ), plus (thf) molecules in the methyl derivative. The structure of the anion shows a Ni-centred NiroSnz icosahedral cage of pseudo-Dsd symmetry surrounded by a polyhedral array of 18 CO groups and two $R$ groups attached to $\mathrm{Sn}$. The methyl derivative has ten terminal, four doubly and four triply bridged CO groups. the butyl derivative has ten terminal, six doubly and two triply bridged Co groups. Despite this difference. the corresponding mean inter-atomic distances in the $\mathrm{Ni}$ centred $\mathrm{Ni}_{10 \mathrm{Sn}}$ cages of the clusters are within $1 \mathrm{pm}$ of each other. The pentagonal antiprism of ten surface $\mathrm{Ni}$ atoms, with an interstitial $\mathrm{Ni}$ and two cappin $\mathrm{Sn}$ atoms has the following mean bond lengths: Ni(central)-Sn, 235. 1(1) pm; Ni(central)-Ni(surface), 258 pm; Ni(surface)-Sn, 272 $\mathrm{pm}$. The intra-pentagonal distance is $\mathrm{Ni}($ surface)-Ni(surface), $279 \mathrm{pm}$, and the intra-pentagonal distance is Ni(surface)-Ni(surface), 251 pm.

The metal framework of $\left[\mathrm{Ni}_{12}\left(\mu_{12}-\mathrm{Sn}\right)(\mathrm{CO})_{12}(\mu-\mathrm{CO})_{0}\left(\mu_{3}-\mathrm{CO}\right)_{4}\right]^{22}[266]$ consists of an icosahedron of $\mathrm{Ni}$ atoms slightly elongated along one pseudo- $\mathrm{C}_{s}$ axis with an interstitial Sn atom. The average interatomic distances in the cluster are: Ni-Ni(intra), $260.6 \mathrm{pm}$; Ni-Ni(inter), $295.6 \mathrm{pm}$; Ni-Ni(cap) 264.1 pm; Sn-Ni(1-5/l'$\left.5^{\prime}\right), 257.5 \mathrm{pm}$ and $\mathrm{Sn}-\mathrm{Ni}(6 / 6), 274.5 \mathrm{pm}$.

There are two white clusters [267] of $\mathrm{Sn}_{3} \mathrm{~W}_{15}$ and $\mathrm{Sn}_{3} \mathrm{~W}_{18}$. The $\left[(\mathrm{PhSn})_{3}\left(\mathrm{P}_{2} \mathrm{~W}_{15} \mathrm{O}_{39} \mathrm{H}_{4}\right)\right]^{5}$ anion is shown in Figure 19. It has the expected $\mathrm{C}_{3 v}$ Dawson structure with three capping $\{\mathrm{WO}\}^{+4}$ units replaced by $\{\mathrm{SnPh}\}^{+3}$.

The $\left[\left(\mathrm{PhSnOH}_{2}\left(\mathrm{PhSnOH}_{2}\right)\left(\mathrm{PW}_{9} \mathrm{O}_{34}\right)_{2}\right]^{-11}\right.$ [267] anion consists of two PW9 anions linked by three Ph3 $\mathrm{Sn}$ groups into an assembly of virtual $\mathrm{D}_{3 \mathrm{~h}}$ symmetry. The $\mathrm{PW}$ g groups are formed by removal of three adjacent corner-shared WO octahedra. The Sn atoms, which act as the filler in a double PW9 sandwich, have slightly distorted octahedral coordination $\left(\mathrm{SnO}_{3} \mathrm{C}\right)$. Each $\mathrm{Sn}$ atom is displaced by $25 \mathrm{pm}$ out of the plane of the four bridging $\mathrm{O}$ atoms in the direction of the phenyl group. 


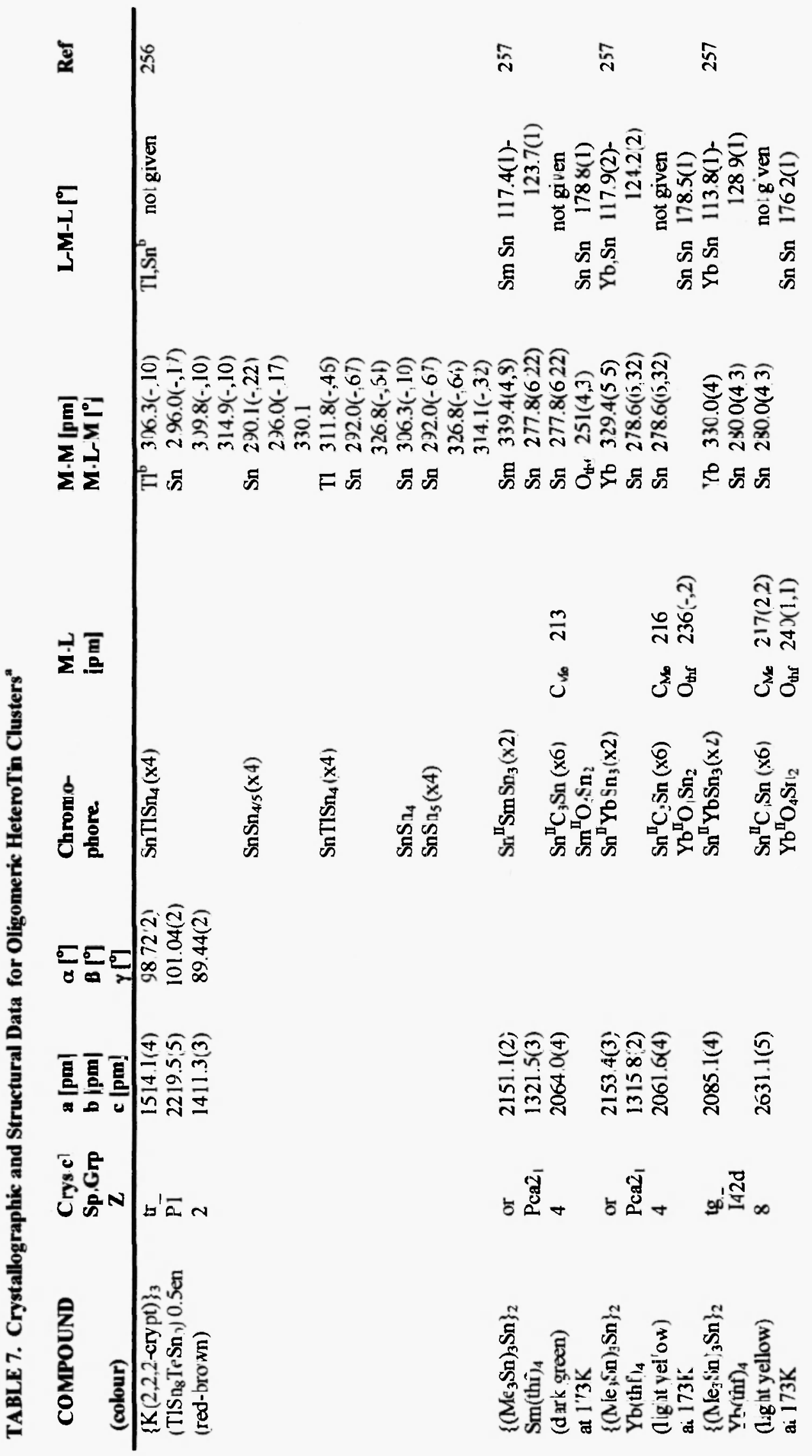




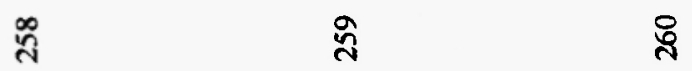

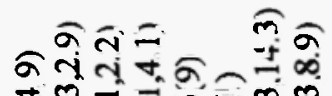

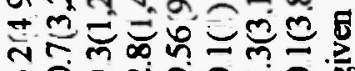

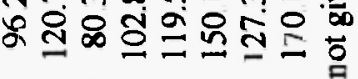

$=0$

거극

จิ

ชัต

을

ธิ㇒ํ.

ป⿻ㅇㅇㅇㅛ

च导导

$\underset{1}{2}$

ซี

Z

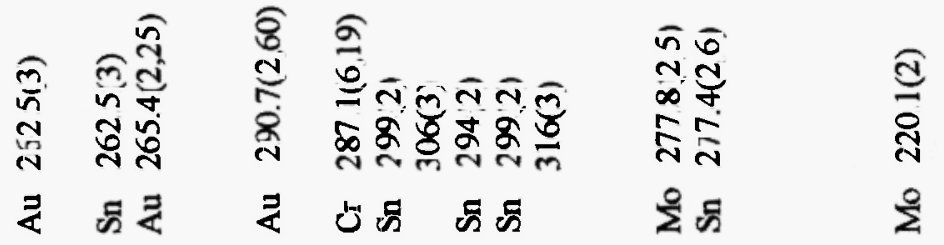

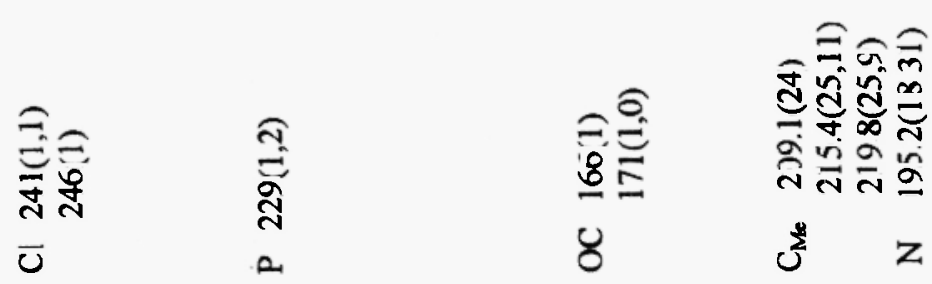

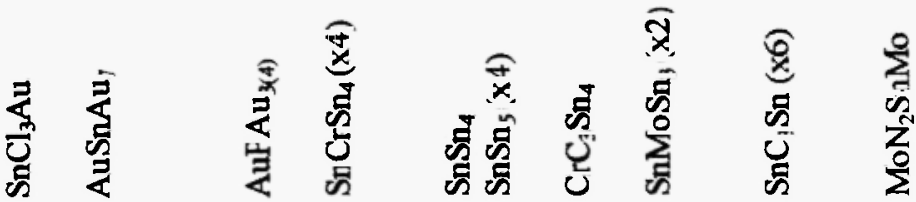

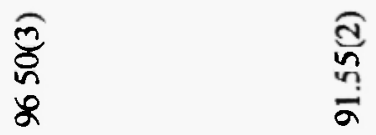

ఫ઼

๙ิ

๖ㅋㅀ

ํํㅇ요웜

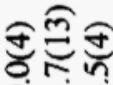

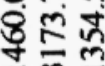

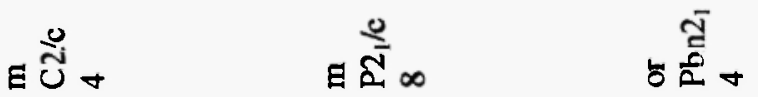
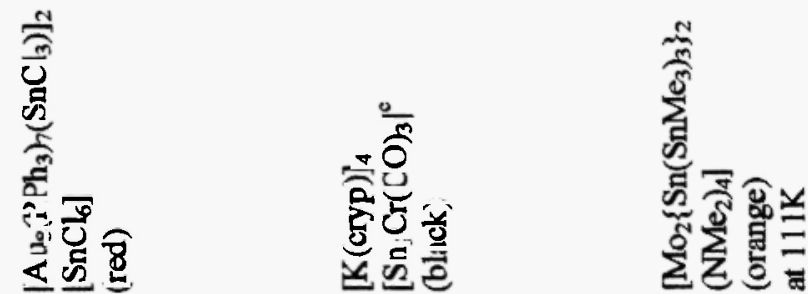
$\bar{i}$

ర్

今ึ

욱

:

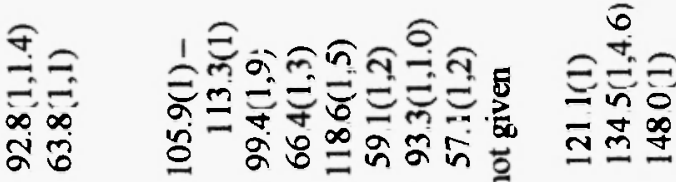

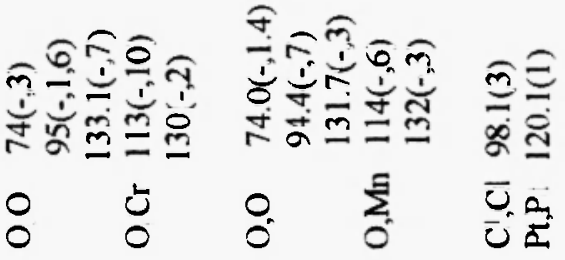

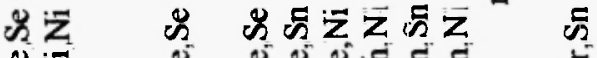

ปี

हñ

ñ.

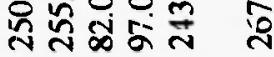

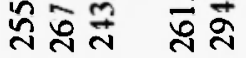

之z z $\bar{z}$

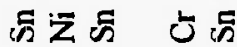

$\underset{\substack{\infty \\ \frac{1}{2}}}{\stackrel{5}{\sim}}$

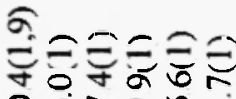

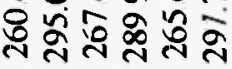

๘

¿ $匚$ ธ

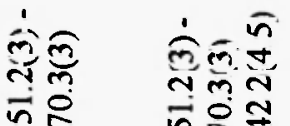

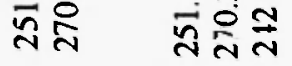

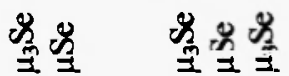

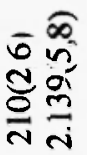

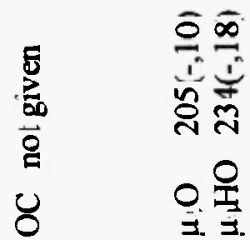

Uी?

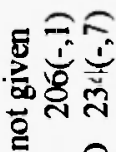

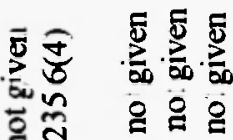

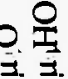

)

छ

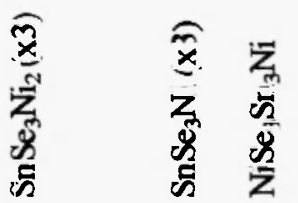

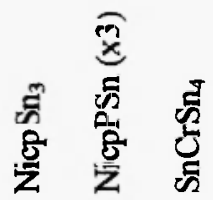

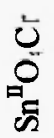

究是

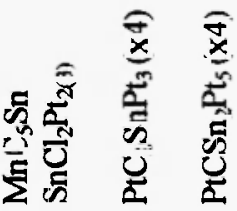

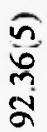

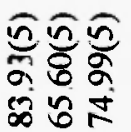

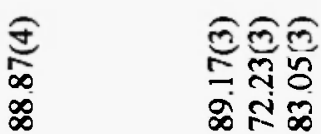

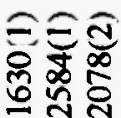

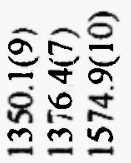

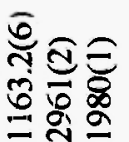

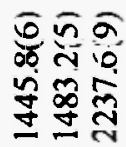

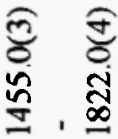

$\Xi \dot{8}$

ロே゙ー

ह

政n

ชูำ

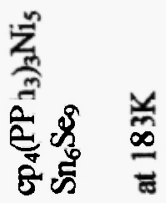
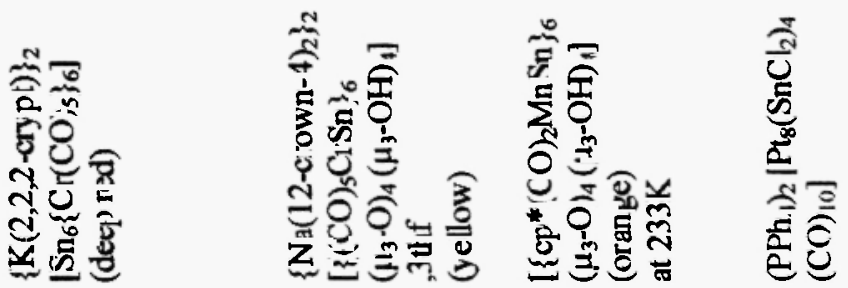


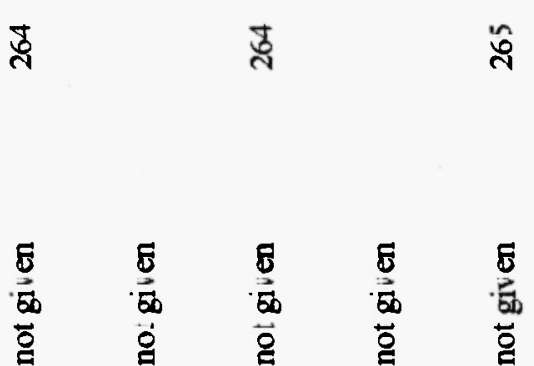

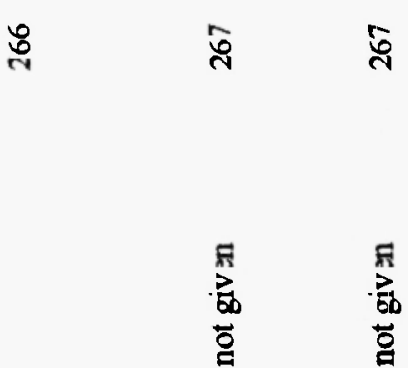

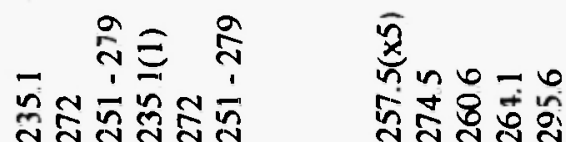

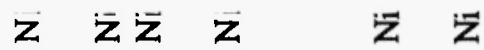

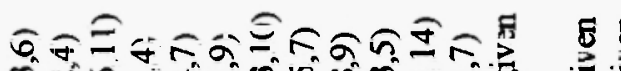

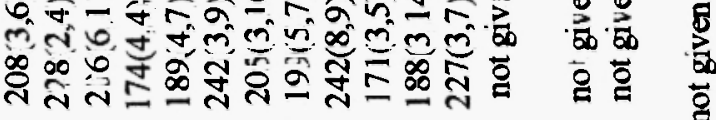

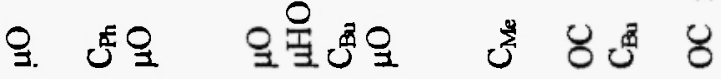

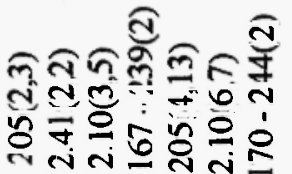

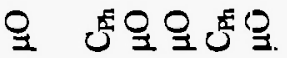

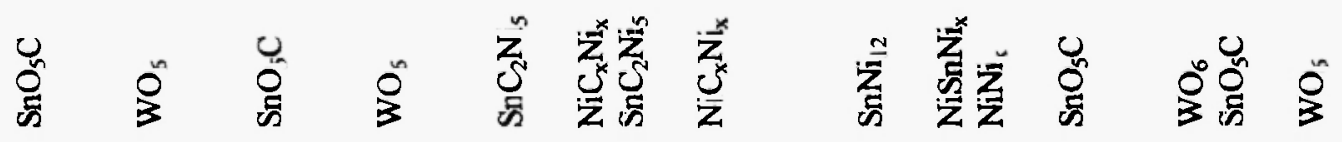

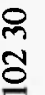

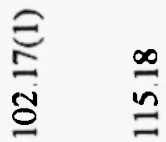

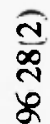

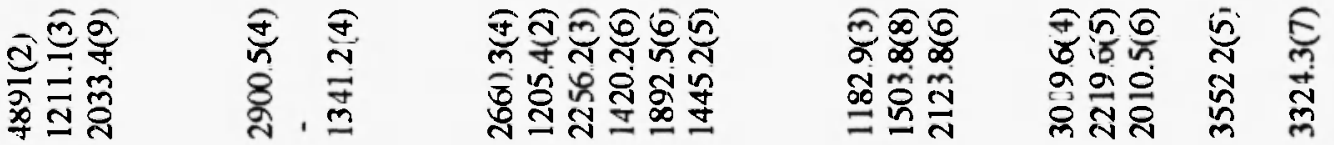

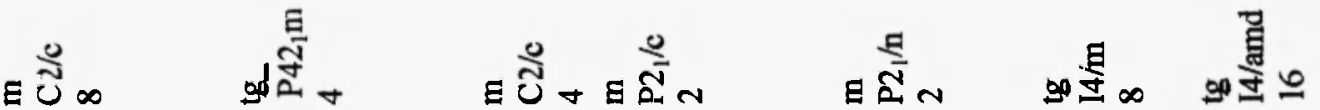

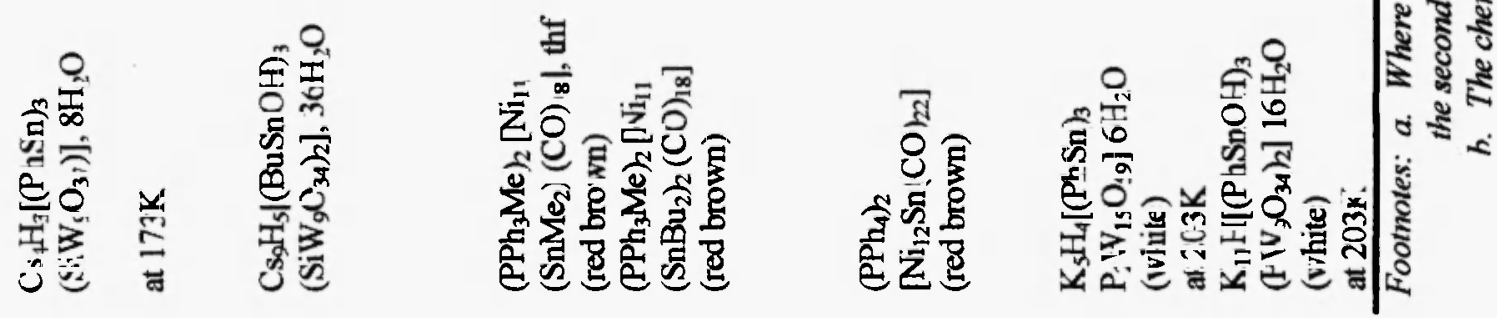




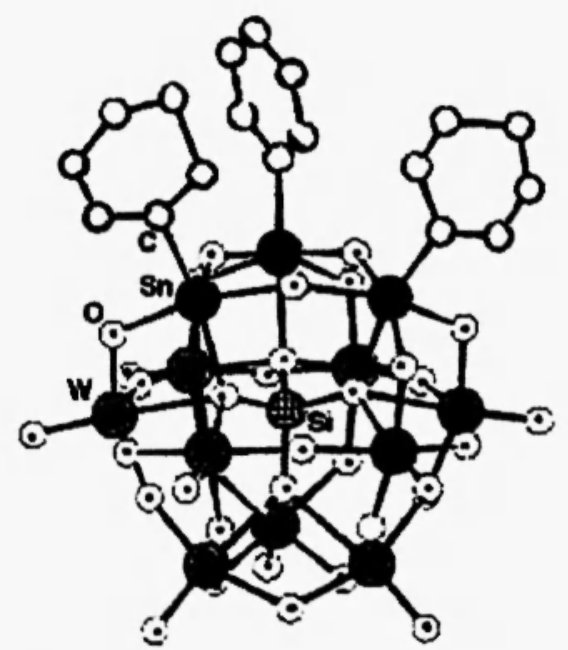

Fig.18. Structure of $[(\mathrm{PhSn}) 3(\beta-S i W, 037)]{ }^{7}[264]$ [267]

The data in Table 7 shows clusters of nine $\mathrm{Sn}_{8} \mathrm{M}(\mathrm{M}=\mathrm{T1}$ [256], Sm or $\mathrm{Yb}$ [257]) and SnAus [258]: ten $\mathrm{Sn} \cdot \mathrm{M}\left(\mathrm{M}=\mathrm{Tl}\right.$ [256] or $\mathrm{Cr}$ [259]) and Sn8Mo2 [260]; eleven $\mathrm{Sn}_{6} \mathrm{Nis}_{6}$ [261]; twelve $\mathrm{Sn}_{6} \mathrm{M}_{6}$ (M = Cr [262] or Mn [240]), Sn4Pt3 [263] and $\mathrm{Sn}_{3} \mathrm{~W}_{9}$ [264]; thirteen $\mathrm{Sn}_{3} \mathrm{Ni}_{11}$ [265] and $\mathrm{SnNi}_{12}$ [266]; eighteen $\mathrm{Sn}_{3} \mathrm{~W}_{15}$ [267], and twenty one $\mathrm{Sn}_{3} \mathrm{~W}_{18}$ [267]. The nonanuclear $\mathrm{Yb}$ derivative [257] has two isomeric forms, orthorhombic and tetragonal, which are isostructural. One example [259] contains two crystallographically independent molecules within the same crystal. Both of these systems are examples of distortion isomerism [113]. The metal-metal distances found in the oligomers are given in Tabel 7A. The Sn-Mo triply bonded distance of 220.192) pm [260] is the shortest in the chemistry of heterometllic tin compounds.

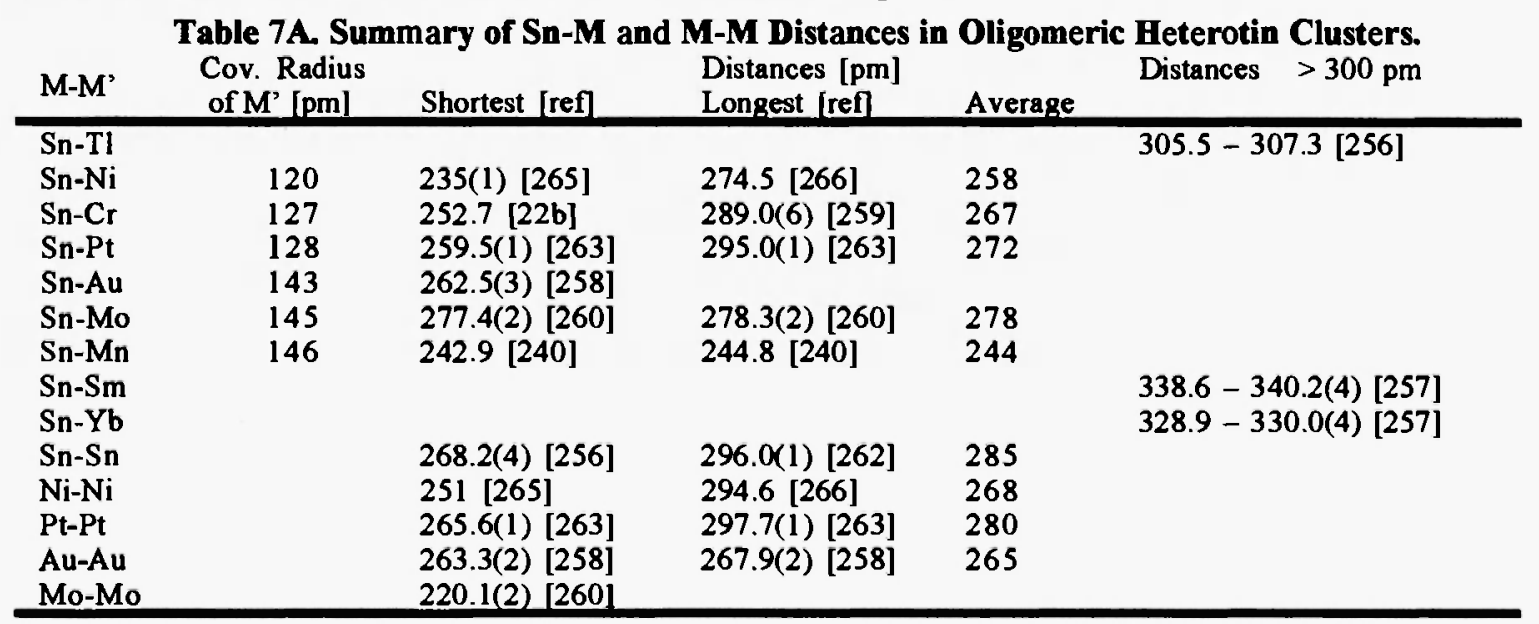

\section{POLYMERIC HETEROTIN COMPOUNDS}

Crystallographic and structural data for almost thirty polymeric heterotin compounds are gathered in Table 8. Both transition and non-transition heterometal atoms are involved in these complexes.

A helical polymer with a Sn(IV) atom in the centre of a trigonal bipyramid of tert-butoxy groups linked by two structurally different atoms has been observed in colourless $[\mathrm{KSn}(\mathrm{OBu}) s(0.5$ tol $)$ [268]. Another colourless complex $\mathrm{KSn}(\mathrm{OBu})_{3}$ [124] is a one dimensional polymer of distorted trigonal bipyramidal $\mathrm{SnO}_{3} \mathrm{M}$ cages aligned to give the central metal atoms a five fold coordination (MOs), as shown Figure 20. The Sn(II) atoms have trigonal pyramidal coordination, with fairly constant Sn-O bond lengths of $206.7 \mathrm{pm}$. The K-O distances range from 256.495 ) to $318.8(6) \mathrm{pm}$. A white $\mathrm{Ca}\left[\mathrm{Sn}(\mathrm{ac})_{3}\right]_{2}$ complex [269] has a three dimensional network structure. The $\mathrm{Ca}$ (II) atom is in distorted octahedral sites. The $\mathrm{Sn}$ (II) atom is at the apex of a regular trigonal pyramid with three equal Sn-O bond distances of 214.0(7) pm and three equal O-Sn-O angles of 


\section{$83.5(2)^{\circ}$.}

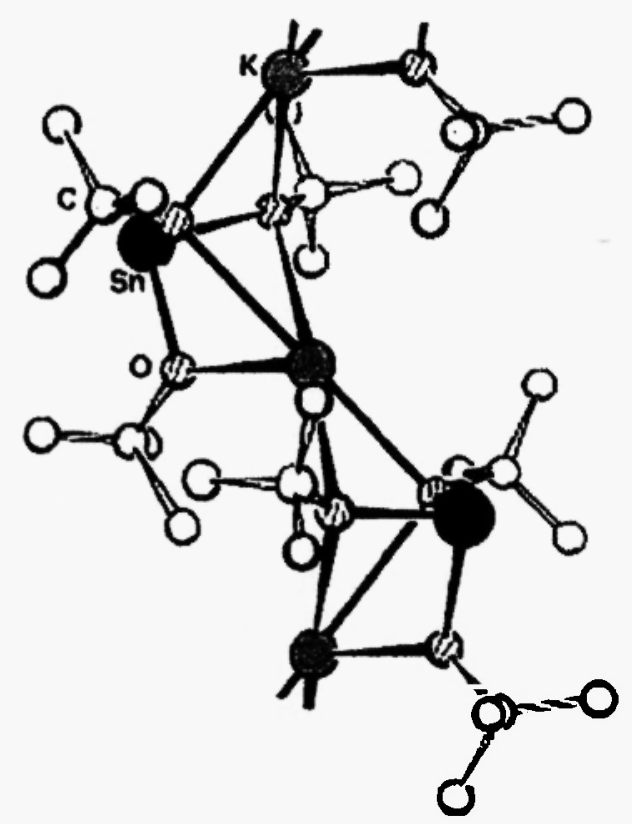

Fig.20. Structure of $\mathrm{KSn}(\mathrm{OBu})$ ] [124]

There are three colourless isostructural (aryl)ClSnAlCl 4 complexes $[170,271]$. All contain the $\mathrm{Sn}_{2} \mathrm{Cl}_{2}{ }^{+2}$ unit as an integral feature. The structures are composed of these dimers interconnected by bridging $\mathrm{AlCl}_{4}$ groups to form an infinite chain structure in [001] directions. Another colourless $\left[\left(\mathrm{C}_{6} \mathrm{H}_{6} \mathrm{Sn}\left(\mathrm{AlCl}_{4}\right)_{2}\right] \mathrm{C}_{6} \mathrm{H}_{6}\right.$ complex [272] is described as $\mathrm{Sn}$ (II) units bonded to two $\mathrm{Cl}$ atoms from three different $\mathrm{AlCl}_{4}$ units to form a linear chain propagating in approximately the [101] direction. In a colourless gallium derivative [273] layers are built up from $\left\{\mathrm{C}_{6} \mathrm{H}_{6} \mathrm{SnCl}\left(\mathrm{GaCl}_{4}\right)\right\}_{2}$ subunits. These consist of a $\mathrm{Sn}_{2} \mathrm{Cl}_{2}$ " four membered ring, two $\mathrm{GaCl}_{4}$ tetrahedra on opposite sides of the ring, and two benzene molecules $\eta^{\circ}$-bonded to the $\mathrm{Sn}$ atoms in a distorted fashion. Colourless $\mathrm{Tl}_{2} \mathrm{Sn}(\mathrm{OEt})_{6}[274]$ exists as a one dimensional polymer of octahedral $\mathrm{Sn}(\mathrm{IV})$ atoms $\left(\mathrm{SnO}_{6}\right)$ and distorted trigonal bipyramidal Tl(I) atoms with the lone pair electrons in the equatorial plane. There are two types of ethoxide group, one with a triply bridging $\mathrm{O}$ atom (between $\mathrm{Sn}$ and two $\mathrm{Tl}$ ), and one with a doubly bridging $\mathrm{O}$ atom (between $\mathrm{Sn}$ and $\mathrm{Tl}$ ).

A structural study of $\left(\mathrm{Bu}^{n}{ }_{4} \mathrm{~N}\right)\left(\mathrm{Et}_{3} \mathrm{Sn}\right)_{2} \mathrm{Cu}$ [275] confirmed the formation of negatively charged, three dimensional, host framework with wide parallel interior channels occupied bywell ordered $\mathrm{Bu}_{4}{ }_{4}$ cations. In the supramolecular aggregate each $\mathrm{Sn}(\mathrm{IV})$ atom has a distorted trigonal bipyramidal environment $\left(\mathrm{SnC}_{3} \mathrm{~N}_{2}\right)$, and the $\mathrm{Cu}(\mathrm{I})$ atoms have a distorted tetrahedral one $\left(\mathrm{CuCl}_{4}\right)$.

There are two yellow [(Me3Sn)4M(CN) 8 ].2thf isostructural polymers $(M=M o$ or W) [276]. Every $\mathrm{M}(\mathrm{CN})$ s group is connected through MesSn bridges to eight other $\mathrm{M}(\mathrm{CN})$ s groups to form the corners of a bodycentred tetragonal unit cell. In another colourless polymer [277] octahedra of trans-Me2 $\mathrm{SnO}_{4}$ and tetrahedra of $\mathrm{MoO}_{4}$ share apices to form a three dimensional structure. Another related system [278] contains tetrahedral $\mathrm{MoO}_{4}$ and trigonal bipyramidal $\mathrm{Me}_{3} \mathrm{SnO}_{2}$ units in which definitive three dimensional structural networks are not found.

Two $\mathrm{Me}_{3} \mathrm{SnMO}_{4}$ polymers $(\mathrm{M}=\mathrm{Te}[279]$ and $\mathrm{Re}[280]$ show differences between each other. Both have a zig-zag chain structure, but the perrhenate complex has a crystallographic mirror axis while the pertechnetate does not. In both compounds the Sn(IV) atom forms the centre of a trigonal bipyramidal coordination geometry, with two $\mathrm{O}$ atoms in apical sites and three methyl groups in the equatorial plane. Both $\mathrm{Te}$ (VII) and Re(VII) atoms have a distorted tetrahedral environment.

Two colourless $\mathrm{Sn} / \mathrm{Fe}$ complexes $[281,282]$ have an infinite, three dimensional, network of two different non-linear chains. These are, A: -(Sn-N-C-Fe-N-Sn-N-C-Fe-C-N-)-; and B: -(Sn-O-O(solvent)O-O-Sn$\mathrm{N}-\mathrm{C}-\mathrm{Fe}-\mathrm{C}-\mathrm{N}-$ )-, where the solvent is $\mathrm{H}_{2} \mathrm{O}$ [281] or 1,4-dioxane [282]. The $\mathrm{Me} 3 \mathrm{Sn}(\mathrm{NC})_{2}$ units of chain $\mathrm{A}$ (average $\mathrm{Sn}-\mathrm{N}=232.0 \mathrm{pm}$ [281] and $233 \mathrm{pm}$ [282]) are essentially trigonal bipyramidal. The Mes $\mathrm{Sn}(\mathrm{NC}) \mathrm{O}$ units of chain $\mathrm{B}$ are also trigonal bipyramidal. The Fe(II) atoms are all six coordinate (FeOt). An orange polymer [283] contains an extensively porous, three dimensional, host framework of corner sharing $\left[\mathrm{Fe}(\mu-\mathrm{CN})_{6}\right]$ and trigonal bipyramidal $\mathrm{SnMe}_{3}(\mu-\mathrm{NC})_{2}$ units. Alternatively, the structure could be viewed as a network of two unique infinite chains, A (non-linear) and B (quasi-linear). An orange-red complex [284] has five coordinate, trigonal 
bipyramidal, $\mathrm{Sn}(\mathrm{IV})$ atoms with equatorial vinyl groups and two apical $\mathrm{O}$ atoms from bridging carboxylate groups from a cyclopentadienyl derivative $\mathrm{C}_{5} \mathrm{H}_{4} \mathrm{COO}$. Their is another cyclopentadienyl ring present, $\mathrm{C}_{5} \mathrm{H}_{5}$, and the result is a linear polymer. Both cyclopentadienyl rings are planar and $\pi$-bonded to the $\mathrm{Fe}$ (II) atom, which is equidistant from these planes.

Distorted $\mathrm{Co}(\mathrm{CNSn})_{6}$ fragments and trigonal bipyramidal configured $\mathrm{N}_{2} \mathrm{SnMe}_{3}$ units are found within the three-dimensional network of a white complex [285]. The structure of a brick red complex $(\mathrm{Me} 3 \mathrm{Sn})_{3} \mathrm{Ru}(\mathrm{CN})_{6}$ [287] is similar to that of its cobalt analogue [286].

The architecture of red $(\mathrm{Me} 3 \mathrm{Sn})_{3} \mathrm{Rh}(\mathrm{SCN})_{6}$ is strongly reminiscent of "super-Prussian blue" systems. There is a three dimensional framework involving $\left\{R_{8}\right\}$ pseudo-cubes as the basic building blocks, with $R h($ III) atoms held apart by non-linear $\{\mathrm{SCN}-\mathrm{SnMe}-\mathrm{NCS}\}$ spacers. The Rh(III) atoms have a quasi-octahedral geometry $\left(\mathrm{RhS}_{6}\right)$. The $\mathrm{Sn}(\mathrm{IV})$ atoms have quasi-trigonal bipyramidal format $\left(\mathrm{SnC}_{3} \mathrm{~N}_{2}\right)$ with $\mathrm{N}$ atoms in apical positions.

The structure of $\mathrm{KSn}(\mathrm{dto})_{3} \mathrm{Cu}\left(\mathrm{Ptol}_{3}\right)_{2}\left(\mathrm{Me}_{2} \mathrm{CO}\right)_{2}$ [289] is shown in Figure 21. The $\mathrm{Sn}(\mathrm{IV})$ atom has a distorted octahedral environment of two $\mathrm{O}$ atoms and four $\mathrm{S}$ atoms from two $\mathrm{S}$,S-chelated and one $\mathrm{O}, \mathrm{O}$-chelated dithiooxalate ligands. The O,O-chelated ligand bridges, via the S,S-chelating side, to the bis(tritolylphosphine)copper(I) moiety. The coordination of the $\mathrm{Cu}$ atom is that of a distorted tetrahedron $\left(\mathrm{CuS}_{2} \mathrm{P}_{2}\right)$. The potassium coordination geometry is an irregular polyhedron formed by seven $\mathrm{O}$ atoms, five from the dithiooxalate ligands and two from acetone molecules. The $\mathrm{K}$ (I) atoms bind the $\left.\mathrm{Sn}(\mathrm{dto})_{3} \mathrm{Cu}(\mathrm{Ptol})_{2}\right)_{2}$ moieties into dimeric pairs which are further linked in chains along the $\mathrm{x}$-axis by the $\mathrm{K}-\mathrm{O}$ linkages.

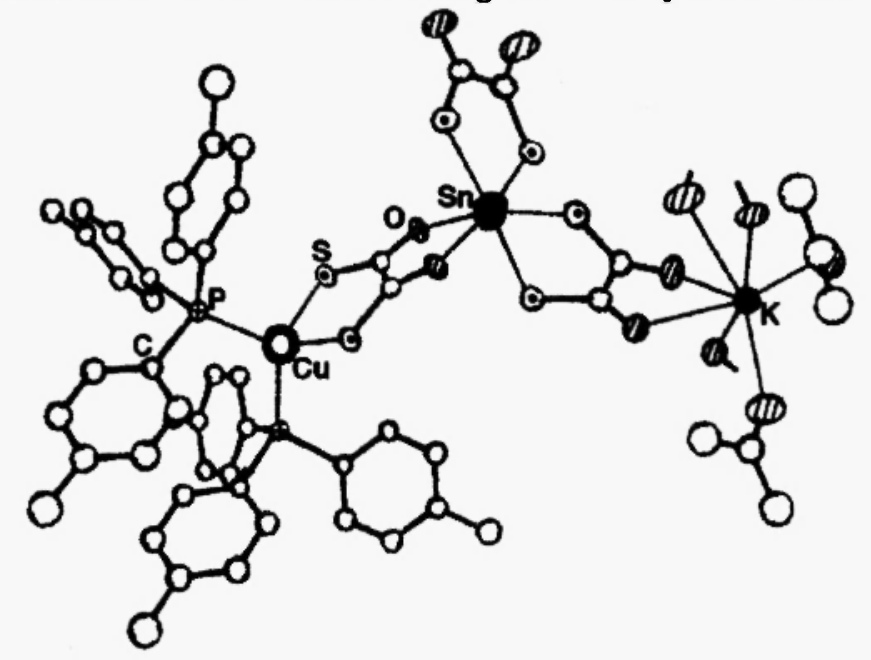

Fig.21. Structure of $\mathrm{KSn}(\mathrm{dto})_{3} \mathrm{Cu}(\mathrm{Ptol})_{2}$ [289].

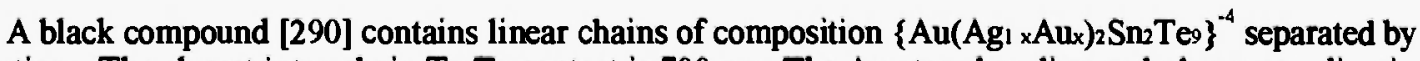
$\mathrm{EN}_{4} \mathrm{~N}^{+}$cations. The closest inter-chain Te-Te contact is $700 \mathrm{pm}$. The Au atom has distorted planar coordination (AuTe) 4 , whereas the Sn and mixed Au/Ag site show tetrahedral and trigonal planar coordination, respectively. A dark red complex [291] contains a mixed metal, one-dimensional, chain with alternating $\left\{\operatorname{In}(\mathrm{en})_{2} \mathrm{Te}_{2}\right\}$ octahedra and $\left\{\mathrm{SnTe}_{1}\right\}$ tetrahedra. For the final Sn(II)-Fe(III) polymer [292] only unit cell dimensions are available.

The data in Table 8 indicate tin in both Sn(II) and Sn(IV) oxidation states, with the latter more common. The Sn(II) atoms are found in pyramidal-planar, trigonal bipyramidal (most common), and seven coordinate geometries. The Sn(IV) atoms are found in tetrahedral, trigonal bipyramidal (again most common) and pseudo-octahedral geometries. The most common ligand donor atoms are $\mathrm{O}$ and $\mathrm{N}$ (as NC). There are no examples with a metal-metal bond, and one and three dimensional chains are the most common.

\section{CONCLUSIONS}

There are over three hundred and eighty heterometallic tin compounds in this review. Of these 130 are trimers, 100 tetramers, 30 pentamers, 45 hexamers, 15 heptamers, 12 octamers, 20 oligomers and 30 polymers. There are about two hundred and forty dimeric derivatives [5], giving a rich chemistry of over six hundred heterometallic derivatives. In these the $\mathrm{Sn}$ atom is found in oxidation states of $0,+2$ and +4 , with geometries including trigonal planar, tetrahedral, trigonal bipyramidal, octahedral and seven coordinate, with varying degrees of distortion. Of these the first only exhibits $\mathrm{Sn}(0)$ and $\mathrm{Sn}$ (II) and the last only $\mathrm{Sn}$ (II) oxidation states. Tetrahedral geometry is by far the most common.

Two clusters $[130,257]$ exist in two isomeric forms with similar stereochemistry, differin by degree of 


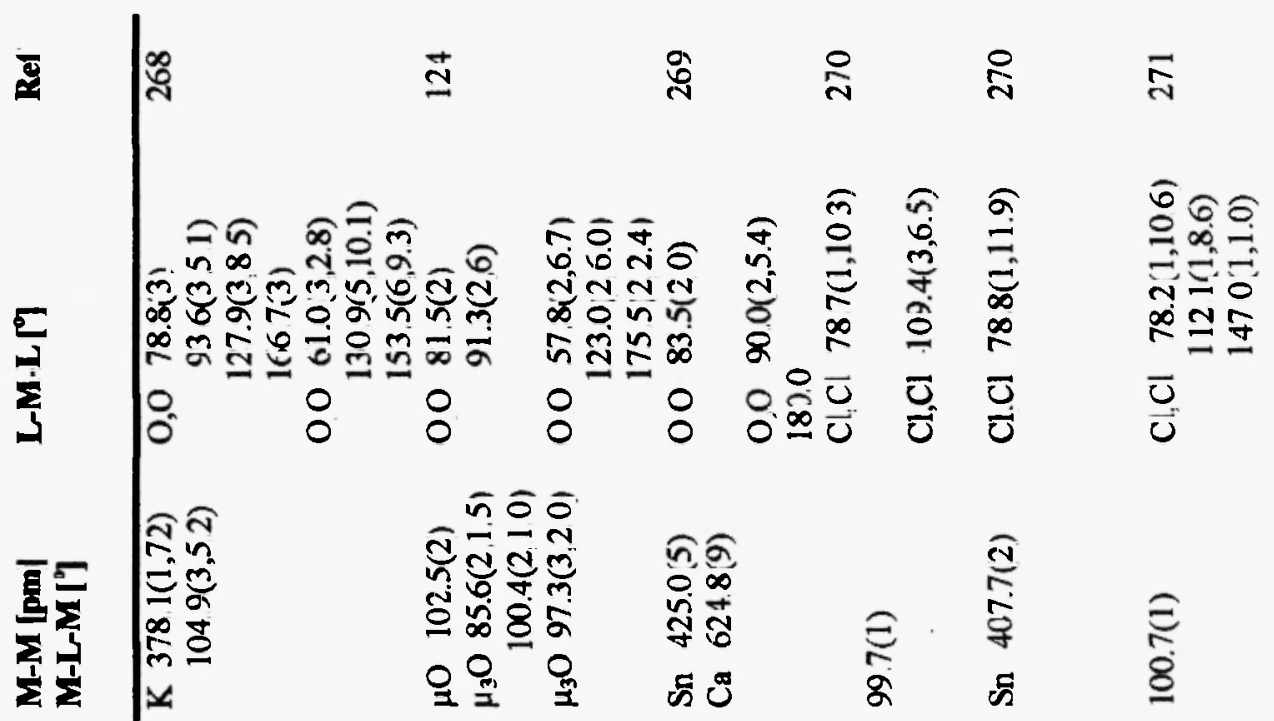

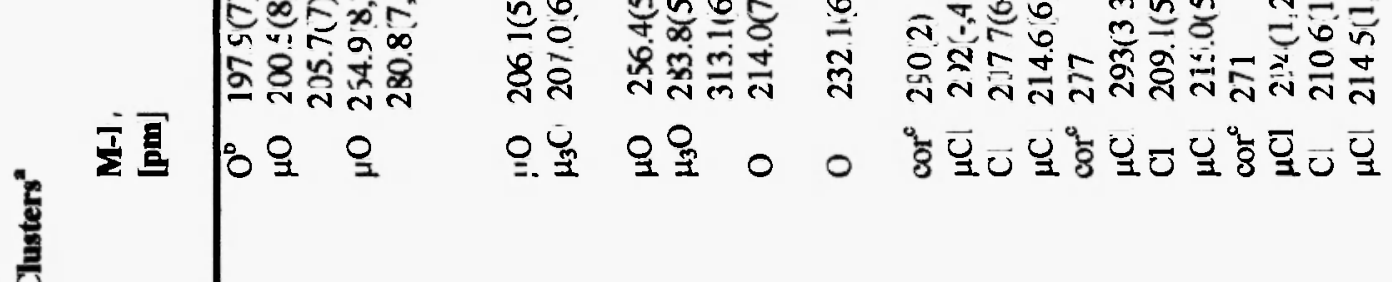

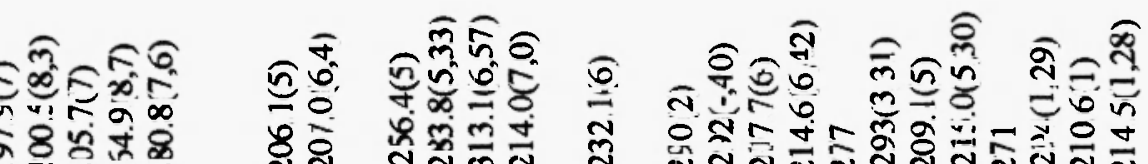

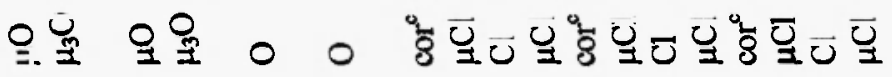

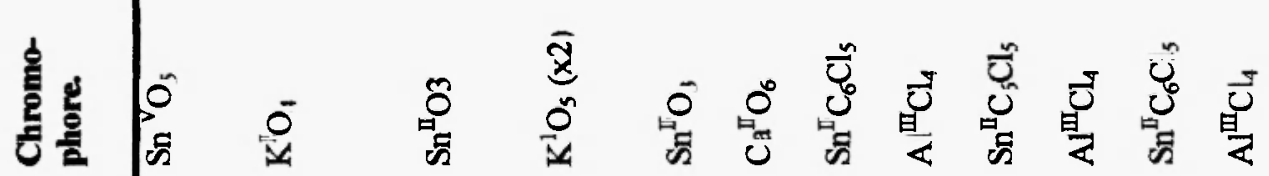
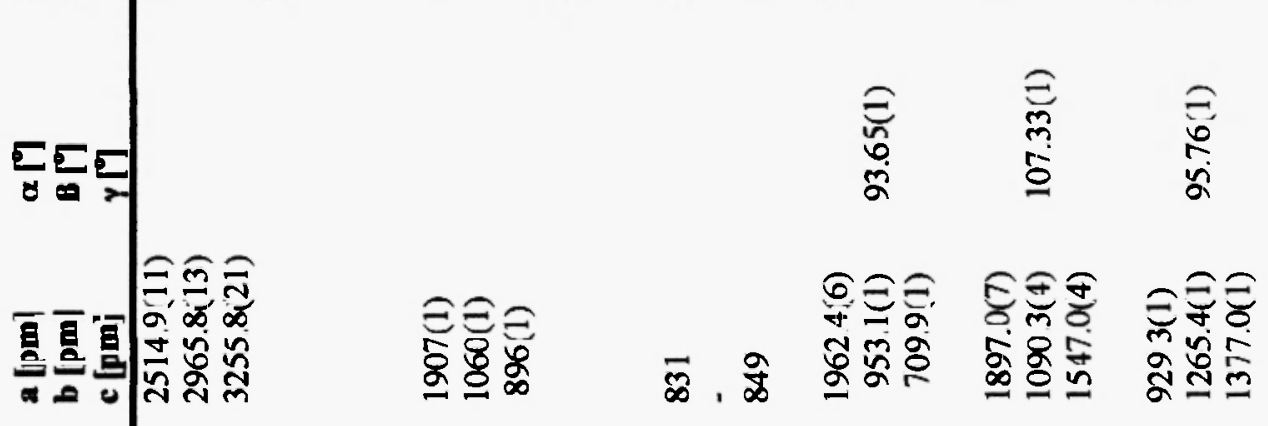

政 SN

5 모에

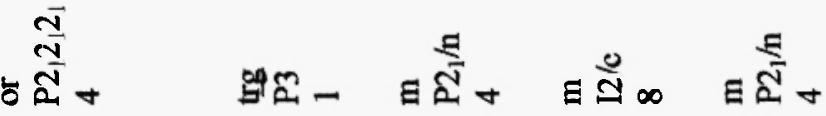

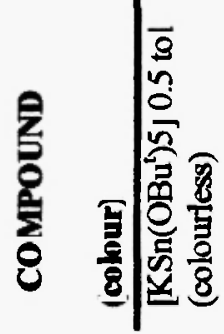

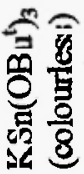

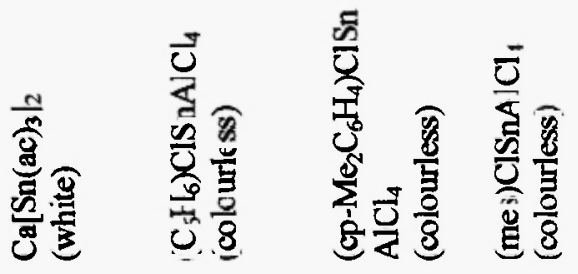



Anahsis of Crystallographic and Structural Data: Part II.

กิ

वे

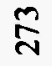

$\stackrel{4}{\stackrel{4}{ }}$

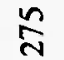

$\stackrel{\text { กิ }}{\stackrel{4}{4}}$

$\stackrel{\infty}{\sim}$

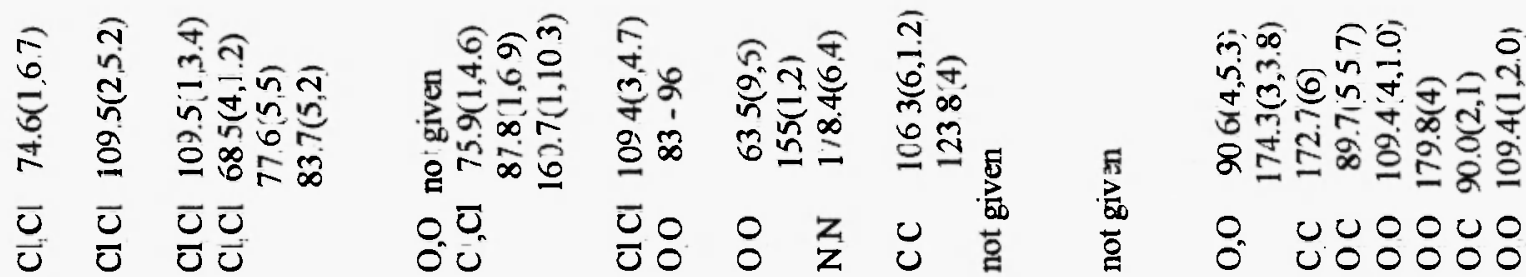

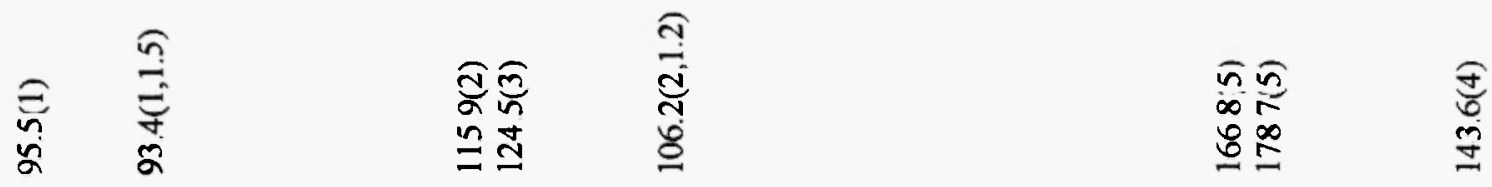

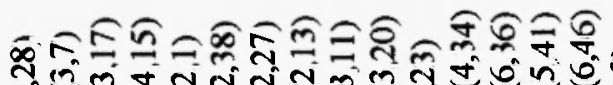

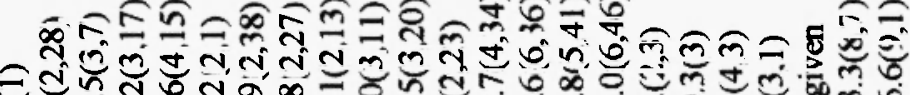

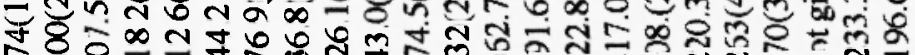

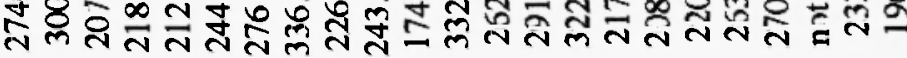

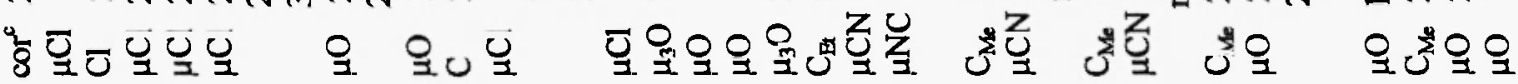

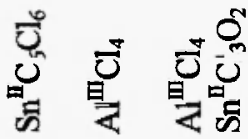

是兽

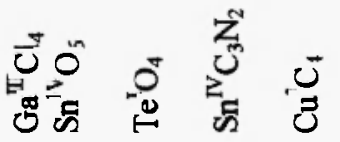

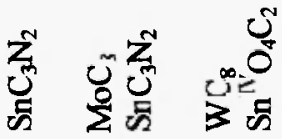

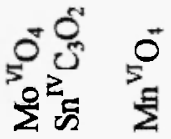

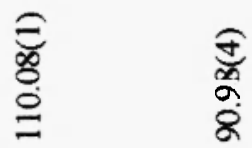

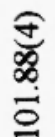

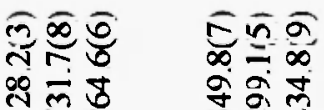

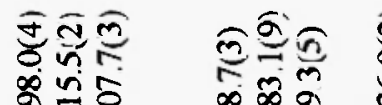

ֻิ

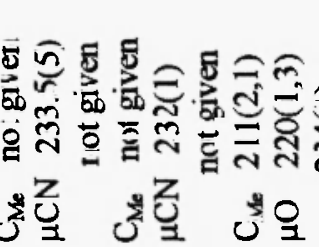

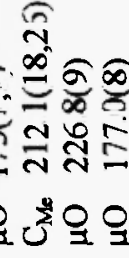

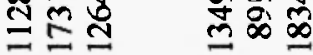

究

我递

$\stackrel{6}{8}$

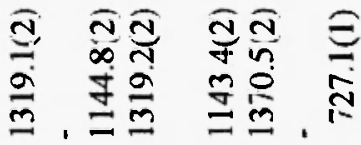

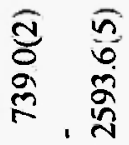

a $\stackrel{\text { กิ }}{\text { a }}$

ฮู

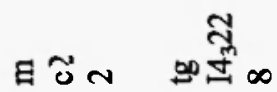<smiles></smiles>

氙

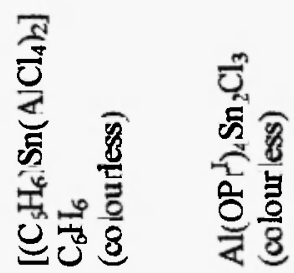

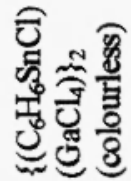

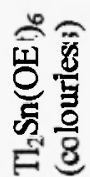
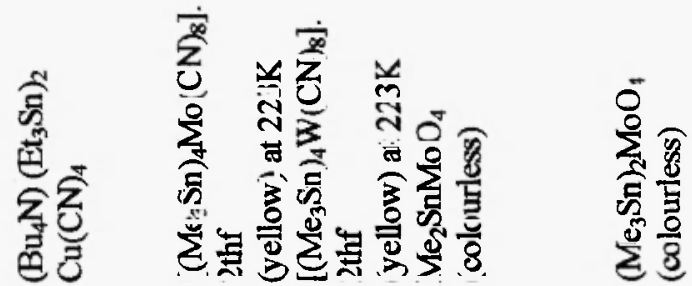


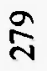<smiles>[Li]</smiles>

$\mathscr{\infty}$

$\stackrel{\infty}{\sim}$

$\vec{\infty}$

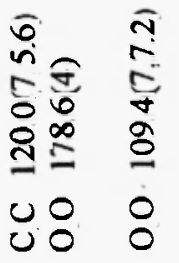

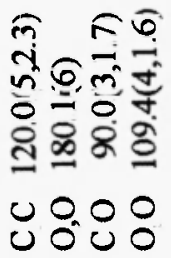

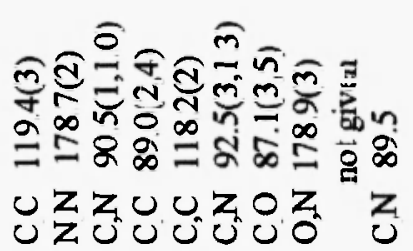

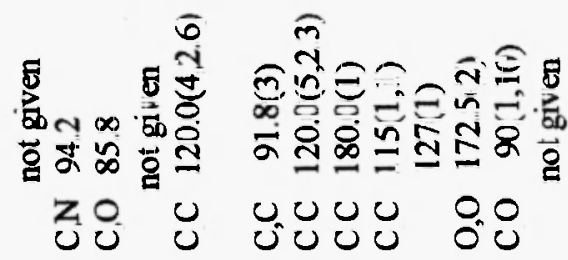

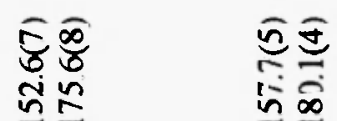

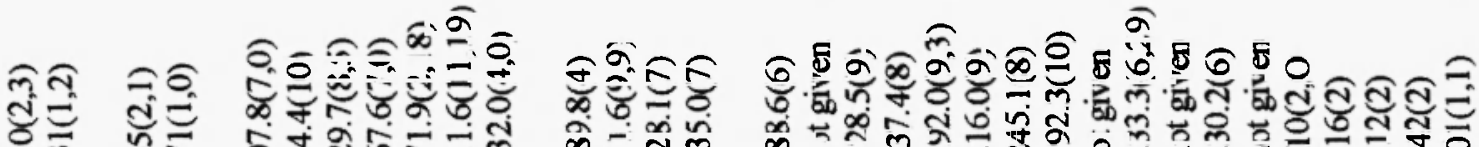

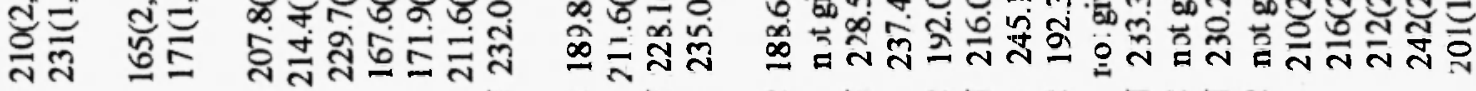

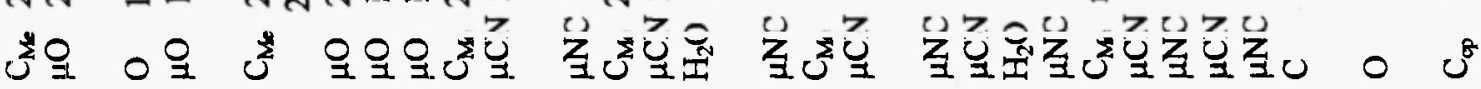

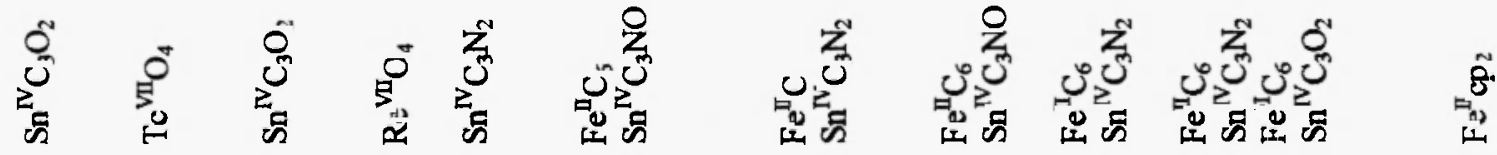
$\frac{2}{6}$

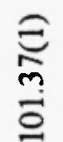

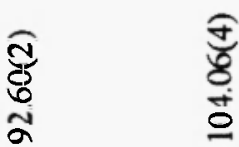

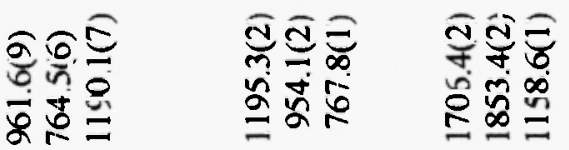

言公

궁

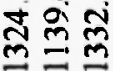

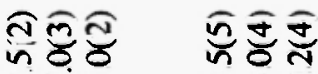

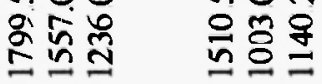

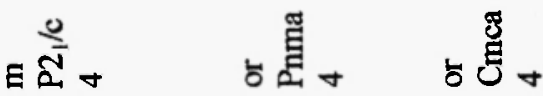

$\frac{a}{2}$

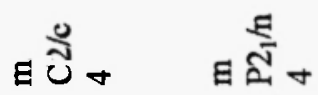

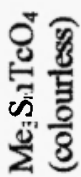
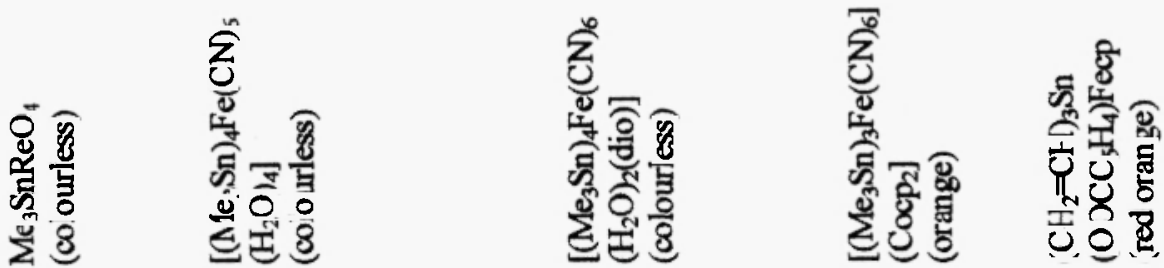


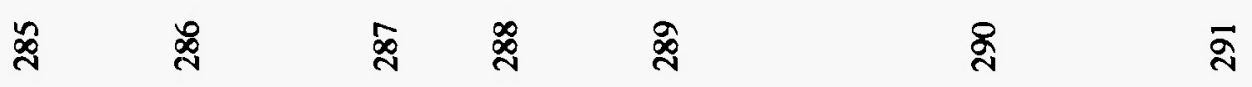

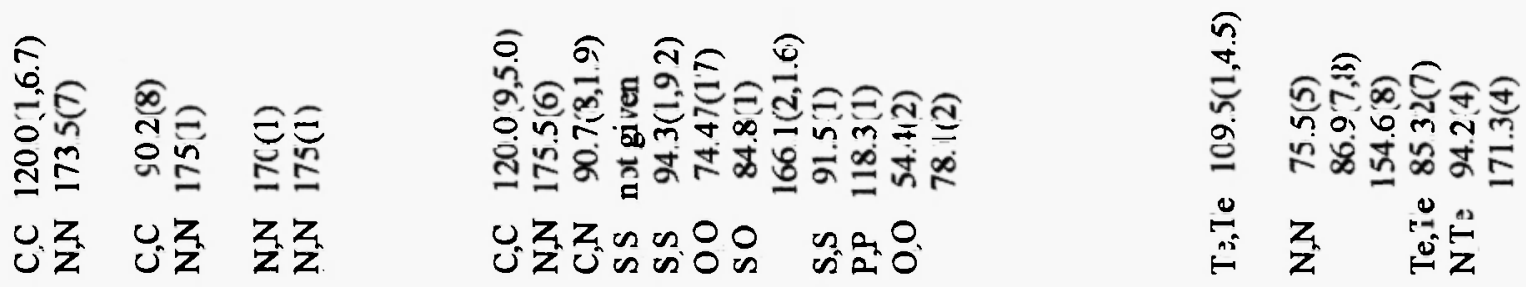

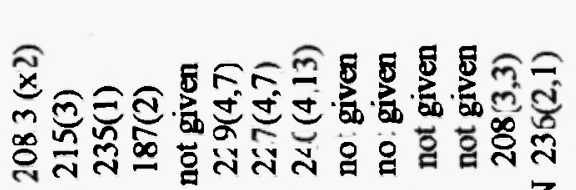

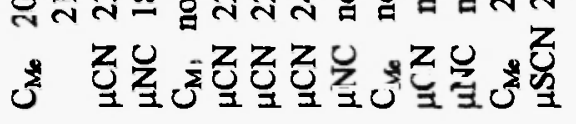

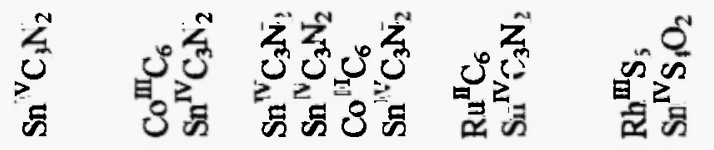

जे

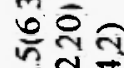

$\infty \underset{\sigma}{\mathbb{\Xi}}$

ते

$\mathbb{R}_{1}^{-\infty}$

บูปิ

85

दू

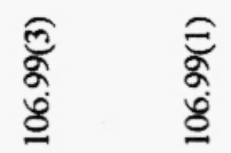

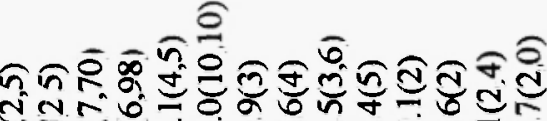

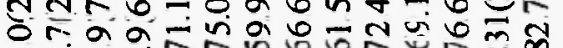

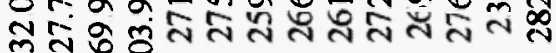

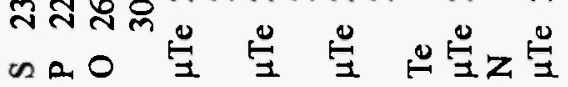

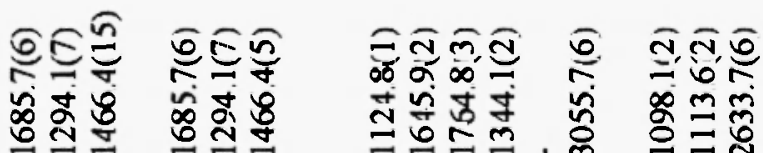

苟

금

$E \Sigma+a \frac{a^{2}}{0}+$

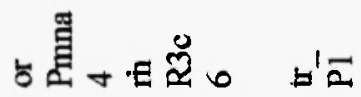

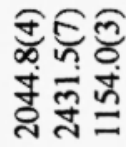

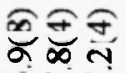

ถ్ูㅚำ

Z⿱

ち莣 


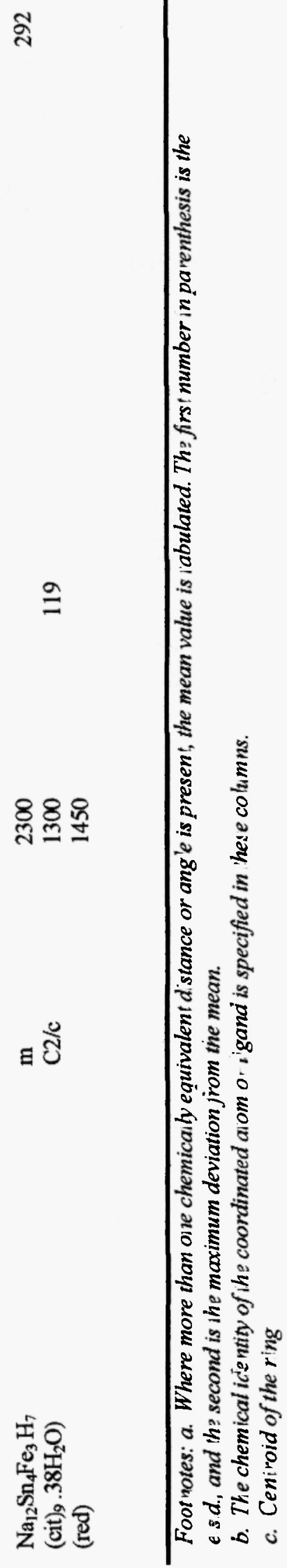


$147,150,167,169,172,179,183,208,209,230,252,259]$ which exhibit distortion isomerism [113], in that they contain two crystallographically independent molecules within the same crystal, differing only by degre of distortion.

The heterometal atoms include the transition and lanthanide metals along with the non-transition metals. The shortest $\mathrm{Sn}-\mathrm{M}$ (non-transition) bond distances are found mostly in the dimeric derivatives [5], with values: 253.7(6) pm (Sn-Ge); 263.4(1) pm (Sn-Zn); 277.6(4) pm (Sn-Li); and 280.9(2) pm (Sn-Pb). In the tetramers the values are: $258.3(3) \mathrm{pm}(\mathrm{Sn}-\mathrm{Mg}) ; 264.8(2) \mathrm{pm}$ (Sn-Ge); and 293.4(6) pm (Sn-Bi). In the hexamers the values are: $278(1) \mathrm{pm}(\mathrm{Sn}-\mathrm{Al})$; and $278.9(1) \mathrm{pm}(\mathrm{Sn}-\mathrm{Pb})$. There is a much richer chemistry with the transition metals, for which the details have been discussed within each sub-section. Only Sn-M distances less than $250 \mathrm{pm}$ are shown in the summary Table 9.

\begin{tabular}{lllllllll}
\multicolumn{2}{c}{ Table 9. Summary of } & Sn-M Bond Diatances In Beterometallic Tin Cluatera \\
Sn-M & Dimers [5] & Trimers & Tetramers & Pentamers & Hexamers & Heptamers & Octamers & Oligomers \\
\hline Sn-Ni & $238.7(1)$ & $232.6(2)$ & $242.0(1)$ & $239.3(2)$ & - & & - & $235(1)$ \\
Sn-Fe & $240.8(1)$ & $249.0(1)$ & $249.9(1)$ & $247.6(1)$ & $248.0(1)$ & $247.1(5)$ & $246.3(1)$ & - \\
Sn-Co & $243.8(1)$ & $244.1(1)$ & - & $246.8(1)$ & - & - & - & - \\
Sn-Pt & $248.6(3)$ & $234.3(1)$ & $247.8(2)$ & - & - & - & - & - \\
Sn-Pd & $247.3(6)$ & - & - & - & - & - & - & - \\
Sn-Rh & $245.0(1)$ & - & - & - & - & - & - & - \\
Sn-Mn & - & $243.7(2)$ & $244.5(1)$ & - & - & $245.5(1)$ & - & 242.9 \\
\hline
\end{tabular}

Although Sn(IV) is formally considered to be "hard" while Sn(II) is borderline "soft", both are found bonded to a variety of "hard" and "soft" heterometal atoms. However, Table 9 indicates that, generally, only the iron subgroup elements generate a direct metal-metal bond with distances less than $250 \mathrm{pm}$. A comparison of shortest $\mathrm{Pb}-\mathrm{M}$ bond distances [293] with $\mathrm{Sn}-\mathrm{M}$ bond distances are given in Table 10. As expected, the $\mathrm{Pb}-\mathrm{M}$ distances are mostly longer than the corresponding $\mathrm{Sn}-\mathrm{M}$ distances, corresponding to the covalent radii of the two Group IV elements (141 pm (Sn) and $147 \mathrm{pm}(\mathrm{Pb})$. However, at 280.9(2) pm the Sn-Pb bond distance is actually shorter than the sum of the respective radii.

Table 10. Summary of the Sn-M and Pb-M [293] bond distances in Heterometal Derivatives.

\begin{tabular}{lllll}
$\mathrm{M}-\mathrm{M}^{\prime}$ & Dimers [pm] [5] & Trimers [pm] & Tetramers [pm] & Pentamers [pm] \\
\hline $\mathrm{Sn}-\mathrm{Ge}$ & $253.7(6)$ & & & \\
$\mathrm{Pb}-\mathrm{Ge}$ & $259.9(2)$ & & & \\
$\mathrm{Sn}-\mathrm{Fe}$ & & $249.0(1)$ & $249.9(1)$ & $247.6(1)$ \\
$\mathrm{Pb}-\mathrm{Fe}$ & & $270.6(5)$ & $262.0(4)$ & $260.5(3)$ \\
$\mathrm{Sn}-\mathrm{Pt}$ & $248.6(3)$ & $234.3(1)$ & & \\
$\mathrm{Pb}-\mathrm{Pt}$ & $269.8(8)$ & $276.9(2)$ & & \\
$\mathrm{Sn}-\mathrm{Li}$ & $277.6(4)$ & & & \\
$\mathrm{Pb}-\mathrm{Li}$ & $285.2(5)$ & & & \\
$\mathrm{Sn}-\mathrm{Ir}$ & & $256.9(3)$ & & \\
$\mathrm{Pb}-\mathrm{Ir}$ & & $283.1(2)$ & & \\
$\mathrm{Sn}-\mathrm{V}$ & $275.7(2)$ & & & \\
$\mathrm{Pb}-\mathrm{V}$ & $293.6(5)$ & & & \\
$\mathrm{Sn}-\mathrm{Mo}$ & & $270.6(1)$ & & \\
$\mathrm{Pb}-\mathrm{Mo}$ & & $280.8(1)$ & & \\
$\mathrm{Sn}-\mathrm{Mn}$ & $250.8(3)$ & $243.7(2)$ & $244.5(1)$ & \\
$\mathrm{Pb}-\mathrm{Mn}$ & $261.7(3)$ & $246.3(1)$ & $249.0(1)$ & \\
$\mathrm{Sn}-\mathrm{Co}$ & & & & $246.8(1)$ \\
$\mathrm{Pb}-\mathrm{Co}$ & & & & \\
$\mathrm{Sn}-\mathrm{Pb}$ & $280.9(2)$ & & & \\
\hline
\end{tabular}

This overview, together with its precursors [1-5], has surveyed nearly three thousand tin compounds. serving to focus attention on area in the rich chemistry of tin which could use additional study. Correlations have been drawn which may assist in prediction of the behaviour of tin in a variety of industrial. biological and environmental situations. In collecting the data it was evident that some data is already irretrievable, some is transparent to data retrieval searches and some is difficult to locate. In a few cases incorrect information has been promulgated in the literature. Where possible these problems have been corrected in this review. Other reviews in this series include magnesium and aluminium, the subgroups of titanium, vanadium, maganese, copper and 
zinc, all of which are referenced in the later reviews of lead $[293,294]$. Also currently underway is a full review of the coordination, organometallic and heterometallic compounds of germanium to complete GroupIV nontransition metals.

\section{ACKNOWLEDGEMENTS}

The authors appreciate the help given by their university libraries, CISTI in Ottawa (Canada) and the British Library at St. Pancras (London, England). The authors wish to thank those who gave permission for reproduction of original figures, the Chemical Faculty of the Slovak Technical University for their cooperation in allowing M.M. to participate, and the Faculty of Pure and Applied Science of York University and the Ministry of Education of the Slovak Republic for financial support.

\section{REFERENCES}

1. C.E. Holloway and M. Melnik, Main Group Metal Chem., 24 (2001) 133.

2. C.E. Holloway and M. Melnik, Main Group Metal Chem., 21 (1998) 371

3. C.E. Holloway and M. Melnik, Main Group Metal Chem., 23 (2000) 1.

4. C.E. Holloway and M. Melnik, Main Group Metal Chem., 23 (2000) 331

5. C.E. Holloway and M. Melnik, Main Group Metal Chem., 23 (2000) 555

6. M. Veith, C. Ruloff, V. Huch and F. Töllner, Angew. Chem. Int. Ed. Engl., 27 (1988) 1381.

7. M. Veith, J. Hans, L Stahl, P. May, V. Huch and A. Sebald, Z. Naturforsch., 46b (1991) 403.

8a. M. Westerhausen, Angew. Chem. Int. Ed. Engl., 33 (1994) 1493.

8b. M. Westerhausen and W. Schwarz, Z. Anorg. Altg. Chem., 622 (1996) 903.

9. M. Veith, D. Kafer and V. Huch, Angew. Chem. Int. Ed. Engl., 25 (1986) 375.

10. M. Westerhausen, H.D. Hausen and W. Schwarz, Z. Anorg. Allg. Chem., 621 (1995) 877.

11. J. Caruso, M.J. Hampden-Smith and E.N. Duesler, J. Chem. Soc., Chem. Commun., (1995) 1041.

12. Kai Ming Chi, S.R. Frerichs and J.E Ellis, J. Chem. Soc., Chem. Commun., (1988) 1013.

13. J.E. Ellis, D.W. Blackburn, Pong Yuen and M. Jang, J. Amer. Chem. Soc., 115 (1993) 11616.

14. J.R. Dilworth, J. Hanich, M. Krestel, J. Beck and J. Străhle, J. Organometal. Chem., 315 (1986) C9.

15. V. Varga, K. Mach, J. Hiller, U. Thewalt, P. Sedmera and M. Polásek, Organometallics, 14 (1995) 1410 .

16. R.M. Whital, G. Ferguson, J.F. Gallagher and W.E. Piers, J. Amer. Chem. Soc., 113 (1991) 9867; W.E. Piers, R.M. Whittal, G. Ferguson, J.F. Gallagher, R.D.J. Froese, H.J. Stronks and P.H. Krygsman, Organometallics, 11 (1992) 4015.

17. J.E. Ellis, Pong Yuen and Meehae Yang, J. Organometal. Chem., 507 (1996) 283.

18. F.G.N. Cloke, K.P. Cox, M.L.H. Green, J. Bashkin and K. Prout, J. Chem. Soc., Chem. Commun,, (1981) 117

19. J.E. Ellis, T.G. Hayes and R.E. Stevens, J. Organometal. Chem., 216 (1981) 191.

20. M.L.H. Green, A.K. Hughes and P. Mountford, J. Chem. Soc., Dalton Trans., (1991) 1699.

21. S.L. Ellis, P.B. Hitchcock, S.A. Holmes, M.F. Lappert and M.J. Slade, J. Organometal. Chem., 444 (1993) 95.

22a. S. Mock and U. Schubert, Chem. Ber., 126 (1993) 2591.

22b. B. Schiemenz, B. Antelmann, G. Hutton and L. Zsolnai, Z. Anorg. Altg. Chem., 620 (1994) 1760.

23. J. Pickardt, H. Schumann, C.F. Campana and L.F. Dahl, J. Organometal. Chem., 216 (1981) 245.

24. V.K. Belski, A.N. Protski, B.M. Bulychev and G.L. Soloveichik, J. Organometal. Chem., 280 (1985) 45.

25. M. Weidenbruch, A. Stilter, W. Sank, K. Peters and H.G. von Schnering, J. Organometal. Chem., 560 (1998) 125.

26. Sang Woo Lee, Keiyuan Yang, J.A. Martin, S.G. Bott and R.G. Richmond, Inorg. Chim. Acta, 232 (1995) 57.

27. M. Akita, T. Oku, M. Tanaka and Y. Moro-Oka, Organometallics, 10 (1991) 3080.

28. M. Knorr, H. Piana, S. Gilbert and U. Schubert, J. Organometal. Chem., 388 (1990) 327.

29. P. Brawnstein, M. Knorr, M. Strompfer, A. DeCian and J. Fischer, J. Chem. Soc., Dalton Trans., (1994) 117.

30. R.K. Pomeroy, L. Vancea, H.P. Calhoren and W.A.G. Graham, Inorg. Chem., 16 (1977) 1508.

31. K. Pannel, J. Castillo-Ramirez and F. Cervantes-Lee, Organometallics, 11 (1992) 3139.

32. M. Herberhold, U. Steffl, W. Milius and B. Wrackmeyer, Angew. Chem. Int. Ed. Engl., 35 (1966) 1803.

33. C. Carini, C. Pelizzi, G. Pelizzi, G. Predieri, P. Tarasconi and F. Vitali, J. Chem. Soc. Chem. Commun. (1990) 613.

34. S.V. Lindeman, Yu.T.Stuchkov and Y.Z. Voloshin, J. Coord. Chem., 28 (1993) 319.

35. B.M. Yamin, Hoong-Kun Fun, Boon-Chuan Yip, O.B. Shawkataly and Siang-Guan Teoh, Acta Crystallogr., Sect. B, 50 (1994) 1551.

36. O.Y. Curnow, B.K. Nicholson and M.J. Severinsen, J. Organometal. Chem., 388 (1990) 379. 
37. Y.Z. Voloshin, V.K. Belsky and V.V. Trachevskii, Polyhedron, 11 (1992) 1939

38. S.V. Lindeman, Y.T. Struchkov and Y.Z. Voloshin, J. Coor. Chem., 34 (1995) 203.

39. M. Veith and L. Stahl, Angew. Chem. Int. Ed. Engl., 32 (1993) 106.

D. Coucouvanis, N.C. Baenziger and S.M. Johnson, J. Amer. Chem. Soc., 95 (1973) 3875.

M.P. Aarnts, M.P. Wilms, K. Peelen, J. Fraanje, K. Goubitz, F. Hartl, D.J. Stufkens, E.J.

Baerends and A. Vlcek, Jr., Inorg. Chem., 35 (1996) 5468.

M. Veith, L. Stahl and V. Huch, Inorg. Chem., 28 (1989) 3278.

B. Czeska, F. Weller and K. Dehnicke, Z. Anorg. Allg. Chem., 498 (1983) 121.

J.P. Collman, D.W. Murphy, E.B. Fleicher and D. Swift, Inorg., Chem., 13 (1974) 1.

A.L. Balch, M.M. Olmstead, D.E. Oram, P.E. Reedy, Jr., and S.H. Reimer, J. Amer. Chem. Soc., $111(1989) 4021$.

47. N.W. Alcock and J.H. Nelson, J. Chem. Soc., Dalton Trans., (1982) 2415.

48. E.N. Yurchenko, T.S. Khodashova, M.A. Porai-Koshits, V.P. Melnikova and V.F. Brattsev. Koord. Khim., 11 (1985) 359; Engl. Ed., p. 19. Amer. Chem. Soc., 104 (1982) 5837.

A. Albinati, P.S. Pregosin and H. Rüegger, Inorg. Chem., 23 (1984) 3223.

L.M. Rendina, J.G. Vittal and R.J. Puddephatt, Organometallics. 15 (1996) 1749.

H. Grützmacher and H. Pritzkow, Chem. Ber., 126 (1993) 2409.

F.G.N. Cloke, C.I. Dalby, P.B. Hitchcock, H. Karamallakis and G.A. Lawless, J. Chem. Soc., Chem., Commun., (1991) 779.

M.N. Bochkarev, V.V. Kharamenkov, Y.F. Radikov, L.N. Zakharov and Y.T. Struchkov, J. Organometal. Chem., 408 (1991) 329. 99. Chem. Ber., 130 (1997) 687.

F.S. Stephens, J. Chem. Soc., Dalton Trans., (1975) 230

61. R.D. Rieke, I. Tucker, S.N. Milligan, D.R. Wright, B.R. Willeford, L.J. Radonovich and M.W. Eyring, Organometallics, 1 (1982) 938. $476(1994) 153$. Chem., 476 (1994) 207.

R.E. Bryan and A.R. Manning, J. Chem. Soc., Chem. Commun., (1968) 1220.

K. Merzweiler, L. Weisse and H. Kraus, Z. Naturforsch., 49b (1994) 425.

M.J. Hampden-Smith, Deqing Lei and E.N. Duesler, J. Chem. Soc., Dalton Trans., (1990) 2953. Feng-Quan Liu, Yi-Tao Wang, Ru-Ji Wang, Hong-Gen Wang and Xin-Kan Yao, J. Organometal. Chem., 371 (1989) 35.

81. Zu-Tao Wang, Xiang-Yang Liu, Xing-Kai Yao and Hong-Gen Wang, J. Organometal. Chem., 331 (1987) 263. 
85. M. Ferrer, O. Rossell, M. Seco, X. Solans and M. Gómez, J. Organometal. Chem., 381 (1990) 183.

86. C.J. Cardin, D.J. Cardin, M.A. Convery, M.M. Devereux, B. Twamley and J. Silver, J. Chem. Soc., Dalton Trans, (1996) 1145.

87. N.G. Bokii, Y.T. Struchkov, V.V. Korol'kov and T.P. Tolstaya, Koord. Khim., 1 (1975) 1144; Engl. Ed., p.965.

88. F.P. Boer and G.J. Flynn, J. Amer. Chem. Soc., 93 (1971) 6495.

89. D. Cunningham, T. Higgins, P. McArdle, W. Corrigan, R. Foley and A.R. Manning, $J$. Organometal. Chem., 481 (1994) 63.

90. P. Klüfers, Z. Naturforsch., 46b (1991) 187.

91. K.M. Mackay, B.K. Nicholson and M. Service, Acta Crystallogr., Sect. C, 46 (1990) 1759.

92. R.D. Hall and D. Hall, J. Organometal. Chem., 56 (1973) 209.

93. E.M. Holt, W. Kläui and J.J. Zucherman, J. Organometal. Chem., 335 (1987) 29.

94. H. Wadepohl, H. Pritzkov and W. Siebert, Organometallics, 2 (1983) 1899.

95. U. Denninger, J.J. Schneider, G. Wilke, R. Goddard and C. Krüger, Inorg. Chim. Acta, 213 (1993) 129.

96. C.J. Cardin, D.J. Cardin, M.A. Convery, Z. Dautier, D. Fenske, M.M. Devereux and M.B. Power, J. Chem. Soc., Dalton Trans., (1996) 1133.

97. Y. Matsuhashi, N. Tokitoh and R. Okazaki, Organometallics, 13 (1994) 4388; N. Tokitoh, Y. Matsuhashi and R. Okazaki, ibid, 12 (1993) 2894.

98. M. Elder and D. Hall, J. Chem. Soc. A, (1970) 245.

99. D.M.T. Chan and T.B. Marder, Angew. Chem. Int. Ed. Engl., 27 (1988) 442.

100. T.A. Wark, E.A. Gulliver, M.J. Hampden-Smith and A.L. Rheingold, Inorg. Chem., 29 (1990) 4360.

101. M.M. Olmstead, L.S. Benner, H. Hope and A.L. Balch, Inorg. Chim. Acta, 32 (1979) 193.

102. A.L. Balch, B.J. Davis and M.M. Olmstead, Inorg. Chem., 29 (1990) 3066.

103. M.N. Bochkarev, V.V. Khramenkov, Y.F. Rad'kov, L.N. Zakharov and Y.T. Struchkov, J. Organometal. Chem., 421 (1991) 29.

104. S. Seebald, B. Mayer and U. Schubert, lnorg. Chem., 34 (1995) 5285.

105. E.C. Onyriuka, S.J. Rettig and A. Storr, Can. J. Chem., 64 (1986) 321.

106. M. Veith and K. Kunze, Angew. Chem. Int. Ed. Engl., 30 (1991) 95.

107. E.M. Löpez, D. Miguel, J.A. Pérez-Martínez, V. Riera and S. Garcia-Granda, J. Organometal. Chem., 492 (1995) 23.

108. D. Miguel, J.A. Perek-Martínez, V. Riera and S. Garcia-Granda, Organometallics, 12 (1993) 2888.

109. B.P. Biryukov, O.P. Solodova and Y.T. Struchkov, Zh. Strukt. Khim., 9 (1968) 228; Engl. Ed., p. 171 .

110. P. Braunstein, M. Knorr, H. Piana and U. Schubert, Organometallics, 10 (1991) 828.

111. P. Braunstein, M. Knorr, M. Strompfer, A. Tiripicchio and F. Ugozzoli, Organometallics, 13 (1994) 3038.

112. M. Melnik, Coord. Chem. Rev., 47 (1982) 239.

113a. R E. Allan, M.A. Beswick, N.L. Cronhout, M.A. Paver, P.R Raithby, A. Steiner, M. Trevithick and D.S. Wright, J. Chem. Soc., Chem. Commun., (1996) 1501.

113b. R. Hillwig, K. Harms and K. Dehnicke, J. Organometal. Chem., 501 (1995) 327.

114. T. Imori, R.H. Heyn, T. Don Tilley and A.L. Rhingold, J. Organometal. Chem., 493 (1995) 83.

115. J.T. Lin, G.P. Hagen and J.E. Ellis, Organometallics, 3 (1984) 1289.

116. R.H. Heyn and T. Don Tilley, Inorg. Chem., 29 (1990) 4051.

117. M.L. Buil, M.A. Esteruelas, F.J. Lahoz, E. Onate and L.A. Oro, J. Amer. Chem. Soc., 117 (1995) 3619.

118. P.B. Hitchcock, M.F. Lappert and M.C. Misra, J. Chem. Soc., Chem. Commun., (1985) 863.

119. E.N. Yurchenko, E.T. Devyatkina, T.S. Khodashova, M.A. Porai-Koshits, V.1. Konnov, V.A. Varnek, P.G. Antonov and Y.N. Kukushkin, Koord. Khim., 5 (1979) 552; Engl. Ed., p.429.

120. M. Grassi, S.V. Meille, A. Musco, R. Pontellini and A. Sironi, J. Chem. Soc., Dalton Trans, (1990) 251.

121. A. Albinati, P.S. Pregosin and H. Rüegger, Angew. Chem. Int. Ed. Engl., 23 (1984) 78.

122. A. Albinati, R. Nägeli, H. Rüegger and P.S. Pregosin, Angew. Chem. Int. Ed. Engl., 21 (1982) 284.

123. R E. Allan, M.A. Beswick, N. Feeder, M. Kranz, M.E.G. Mosquera, P.R. Raithby, A.E.H. Wheatley and D.S. Wright, Inorg. Chem., 37 (1998) 2602.

124. M. Veith and R. Rösler, Z. Naturforsch., $41 \mathrm{~b}$ (1986) 1071.

125. A. Steiner and D. Stalke, J. Chem. Soc., Chem. Commun., (1993) 1702.

126. M. Veith, D. Käfer, J. Koch, P. May, L. Stahl and V. Huch, Chem. Ber., 125 (1992) 1033.

127. S.U. Ghazi, R. Kumar, M.J. Heeg and J.P. Oliver, Inorg. Chem., 33 (1994) 411.

128. H. Schmidbaur, T. Probst, B. Huber, O. Steigelmann and G. Müller, Organometallics, 8 (1989) 1567. 
129.

130.

131.

132.

133.

134.

135.

136.

137.

138.

139.

140.

141.

142.

143.

144.

145.

146.

147.

148.

149.

150.

151.

152.

153.

154.

155.

156.

157.

158.

159.

160.

161.

$162 \mathrm{a}$.

$162 \mathrm{~b}$.

163.

164.

165.

166.

167.

168.

169.

170.

171.

172.

173.

174.

175.

H. Schmidbaur, T. Probst and O. Steigelmann, Organometallics, 10 (1991) 3176.

W. Frank, Z. Anorg. Altg. Chem., 585 (1990) 121.

W.R. Nutt, K.J. Murray, J.M. Gulick, J.O. Odom, Yan Ding and L. Lebioda, Organometallics, 15 (1996) 1728.

H.D. Hausen, R. Kuhnle and J. Weidlein, Z. Naturforsch., 50b (1995) 1419.

S.U. Ghazi, M.J. Heeg and J.P. Oliver, Inorg. Chem., 33 (1994) 4517.

C. Leue, R. Reau, B. Neumann, H.G. Stammler, P. Jutzi and G. Bertrand, Organometallics, 13 (1994) 436.

S.C. Critchlow and J.D. Corbett, Inorg. Chem., 21 (1982) 3286.

Yuniu Bai, H.W. Roesky, H.G. Schmidt and M. Noltemeyer, Z. Naturforsch., 47b (1992) 603.

Feng-Quan liu, A. Herrog, H.W. Roesky and I. Uson, Inorg. Chem., 35 (1996) 741.

M.L.H. Green, A.K. Hughes and P. Mountford, J. Chem. Soc., Dalton Trans., (1991) 1407.

B. Krebs, B. Lettmann, H. Pohlmann and R. Frőhlich, Z. Kristallogr., 196 (1991) 231.

R. Carreno, V. Riera, M.A. Ruiz, Y. Yeannin and M. Philoche-Levisalles, J. Chem. Soc., Chem. Commun., (1990) 15.

B.A. Narayanan and J.K. Kochi, Inorg. Chim. Acta, 122 (1986) 85.

C.J. Gilmore and P. Woodward, J. Chem. Soc., Dalton Trans., (1972) 1387.

P. Braunstein, C. Charles, R.A. Adams and R. Layland, J. Cluster Sc., 7 (1996) 145.

P.G. Harrison, T.J. King and J.A. Richards, J. Chem. Soc., Dalton Trans., (1975) 2079.

R. Restivo and R.F. Bryan, J. Chem. Soc. A, (1971) 3364.

M. Veith, L. Stahl and V. Huch, Organometallics, 12 (1993) 1914.

S. Sharma, J. Cervantes, J.L. Mata-Mata, M.C. Brun, F. Cervantes-Lee and K.H. Pannell, Organometallics, 14 (1995) 4269.

A. Clearfield, C.J. Simmons, E.P. Withers, Jr., and D. Seyferth, Inorg. Chim. Acta, 75 (1983) 139.

Teng-Yuan Dong, Ming-Yhu Hwang, Yuh-Sheng Wen and Wen-Shu Hwang, J. Organometal. Chem., 391 (1990) 377.

J. Weaver and P. Woodward, J. Chem. Soc., Dalton Trans., (1973) 1060.

H. Kranz and K. Merzweiler, Z. Naturforsch., 52b (1997) 635.

J.A.K. Howard, S.C. Kellett and P. Woodward, J. Chem. Soc., Dalton Trans., (1975) 2332.

A.L. Balch, H. Hope and F.E. Wood, J. Amer. Chem. Soc., 107 (1985) 6936.

Weng Kee Leong, F.W.B. Einstein and R.K. Pomeroy, Organometallics, 15 (1996) 1582.

A.L. Balch, K.M. Waggoner and M M. Olmstead, Inorg. Chem., 27 (1988) 4511.

M.T. Pinillos, A. Elduque, J.A. Lopez, F.J. Lahoz, L.A. Oro and B.E. Mann, J. Chem. Soc., Dalton Trans., (1992) 2389.

A. Albinati, R. Nargeli, K.H.A. Ostoja Starzewski, P.S. Pregosin and H. Rüegger, Inorg. Chim. Acta, 76 (1983) L231.

H.C. Clark, G. Ferguson, A.B. Goel and B.L. Ruhl, Organometallics, 3 (1984) 15.

P. Kircher, G. Huttner and K. Heinze, J. Organometal. Chem., 562 (1998) 217.

J.E. Varey, G.J. Lamprecht, V.P. Fedin, A. Holder, W. Clegg, M.R.J. Elsegood and A.G. Sykes, Inorg. Chem., 35 (1996) 5525.

H. Keck, A. Kruse, W. Kuchen, D. Mootz, R. Wiskemann and H. Wunderlich, Z. Naturforsch., 45b (1990) 461. (2). Sc.

A. Müller, V.P. Fedin, E. Diemann, H. Bögge, E. Krict
Barbieri and P. Adler, Inorg. Chem., 33 (1994) 2243.

W.A. Hermann, H.J. Kneuper and E. Herdweck, Chem. Ber., 122 (1989) 437.

H. Preut and H.J. Haupt, Acta Crystallogr., Sect. B, 37 (1981) 688.

H. Preut and H.J. Haupt, Acta Crystallogr., Sect. $B, 38$ (1982) 1290.

H.J. Haupt, H. Preut and W. Wolfes, Z. Anorg. Allg. Chem., 446 (1978) 105.

B.T. Huie, S. W. Kirtley, C.B. Knobler and H.D. Kaesz, J. Ónganometal. Chem., 213 (1981) 45.

G.B. Hitchcock, M.F. Lappert and M.J. McGeary, Organometallics, 9 (1990) 884.

J.M. Cassidy and K.H. Whitmire, Inorg. Chem., 28 (1989) 2494.

R.D. Ball and D. Hall, J. Organometal. Chem., 52 (1973) 293.

B.P. Biryukov, E.A. Kukhtensova, Y.T. Struchkov, K.N. Anisimov, N.E. Kolobova and V.I. Khandozhko, J. Organometal. Chem., 27 (1971) 337.

R.D. Adams and D.A. Katahira, Organometallics, 1 (1982) 53

J.A. Cabeza, S. Garcia-Granda, A. Llamazares, V. Rièrā and J.F. Van der Maelen, Organometallics, 12 (1993) 157.

C.J. Cardin, D.J. Cardin, M.A. Convery and M.M. Devereux, J. Chem. Soc., Chem. Commun., (1991) 687.

N. Viswanathan, E.D. Morrison, G.L. Geoffrey, S.J. Geib and A.L. Rheingold, Inorg. Chem., 25 (1986) 3100 . 
176. C.J. Cardin, D.J. Cardin, J.M. Power and M.B. Hursthouse, J. Amer. Chem. Soc., 107 (1985) 505.

177. C.J. Cardin, D.J. Cardin, H.E. Parge and J.M. Power, J. Chem. Soc., Chem. Commun., (1984) 609.

178. R.D. Adams and D.A. Katakira, Organometallics, 1 (1982) 460.

179. R. Uson, J. Formes, M. Tomas and I. Uson, Angew. Chem., Int. Ed. Engl., 29 (1990) 1449.

180. G. Douglas, M.C. Jennings, L. Manojlovic-Muir, K.W. Muir and R.J. Puddephatt, J. Chem Soc., Chem. Commun., (1989) 159.

181. S. Onaka, Y. Kondo, K. Toriumi and T. Ito, Chem. Letters (1980) 1605; S. Onaka, Y. Kondo, M. Yamashita, Y. Tatematsu, Y. Kato, M. Goto and T. Ito, Inorg. Chem., 24 (1985) 1070.

182. J.E. O'Connor and E.R. Corey, J. Amer. Chem. Soc., 89 (1967) 3930.

183. M. Moll, H. Behrens, P. Merbach, K. Görting, G. Liehr and R. Böhme, Z. Naturforsch', 35b (1980) 1115.

184. F.H. Carre, R.J.P. Corriu and B.J.L. Henner, J. Organometal. Chem., 228 (1982) 139.

185. M. Veith and O. Recktenwald, Z. Naturforsch., 36b (1981) 144.

186. J.E. Ellis, Kai-Ming Chi, A.J. Di Maio, S.R. Frerichs, J.R. Stenzel, A.L. Rheingold and B.S. Haggerty, Angew. Chem. Int. Ed. Engl., 30 (1991) 194.

187. G.L. Rochfort and J.E. Ellis, J. Organometal. Chem., 250 (1983) 277.

188. M. Veith, L. Stahl and V. Huch, J. Chem. Soc., Chem. Commun., (1990) 359.

189. C.S. Velazquez, W.E. Broderick, M. Sabat, A.G.M. Barrett and B.M. Hoffman, J. Amer. Chem. Soc., 112 (1990) 7408; C.S. Velázquez, A.G.M. Barrett and B.M. Hoffman, ibid, 114 (1992) 7416.

190. J.F. Almeida, K.R. Dixon, C. Eaborn, P.B. Hitchcock, A. Pidcock and J. Vinaixa, J. Chem. Soc., Chem. Commun., (1982) 1315.

191. G.D. Smith, P.E. Fanwick and I.P. Rothwell, Acta Crystallogr., Sect. C, $5 I$ (1995) 2501.

192. C.J. Cardin, D.J. Cardin, G.A. Lawless, J.M. Power, M.B. Power and M.B. Hursthouse, J. Organometal. Chem., 325 (1987) 203.

193. V.V. Bashilov, V.J. Sokolov, Y.L. Slovokhotov, Y.T. Struchkov, E.G. Mednikov and N.K. Eremenko, Dokl. Akad. Nauk SSSR. 292 (1987) 863; J. Organometal. Chem., 327 (1987) 285.

194. R.A. Bartlett, C.J. Cardin, D.J. Cardin, G.A. Lawless, J.M. Power and P.P. Power, J. Chem. Soc., Chem. Commun., (1988) 312.

195. F.W.B. Einstein, R.K. Pomeroy and A.C. Willis, J. Organometal. Chem., 311 (1986) 257.

196. L.J. Guggenberger, J. Chem. Soc., Chem. Commun., (1968) 512.

197. M.C. Jennings, G. Schoettel, S. Roy, R.J. Puddephatt, G. Douglas, L. Manojlovic-Muir and K.W. Muir, Organometallics, 10 (1991) 580.

198. R.E. Allen, A. Bashall, J.S. Palmer, M. McPartlin, M.E.G. Mosquera, J.M. Rawson, A.E.H. Wheatley and D.S. Wright, J. Chem Soc., Chem. Commun., (1997) 1975.

199. D.E. Westerberg, B.R. Sutherland, J.C. Huffman and K.G. Caulton, J. Amer. Chem. Soc., 110 (1988) 1642.

200. J.M. Cassidy, K.H. Whitmire and A.M. Kook, J. Organometal. Chem., 456 (1993) 61.

201. J.S. Leigh and K.H. Whitmire, Acta Crystallogr., Sect. C, 46 (1990) 732.

202. A. Cabrera, H. Samain, A. Mortneux, F. Petit and A.J. Welch, Organometallics, 9 (1990) 959.

203. B. Wrackmeyer, H.E. Maisel, G. Kehr and H. Nöth, J. Organometal. Chem., 532 (1997) 200.

204. Chang-Yao Lu, F.W.B. Frederick, W.B. Einstein, V.J. Johnson and R.K. Pomeroy, Inorg. Chem., 28 (1989) 4212.

205. D.B. Firfiray, A. Irving and J.R. Moss, J. Amer. Soc., Chem. Commun., (1990) 377.

206. M. Veith and J. Hans, Angew. Chem. Int. Ed. Engl., 30 (1991) 878.

207. L.J. Farrugia, B.R. James, C.R. Lassigne and E.J. Wells, Can. J. Chem., 60 (1982) 1304; Inorg. Chim. Acta, 53 (1981) L261.

208. E.N. Yurchenko, E.T. Devyatkina, T.S. Khodashova, M.A. Porai-Koshits, V.I. Konnov, V.A. Varnek, P.G. Antonov and Y.N. Kukushkin, Koord. Khim., 5 (1979) 552; Engl. Ed., p.429.

209. T.S. Khodashova, M.A. Porai-Koshits, V.P. Nikolaev and V.N. Shchurkina, Z. Strukt. Khim., 21 (1980) 123; Engl. Ed., 220.

210. J.H. Nelson and N.W. Alcock, Inorg. Chem., 21 (1982) 1196.

211. M. Veith, P. Hobein and V. Huch, J. Chem. Soc., Chem. Commun., (1995) 213.

212. M. Veith and W. Frank, Angew, Chem. Int. Ed. Engl., 24 (1985) 223.

213. S. Roller, M. Dräger, H.J. Breunig, M. Ates and S. Gülec, J. Organometal. Chem., 329 (1987) 319.

214. G. Becker, M. Meiser, O. Mundt and J. Weidlein, Z. Anorg. Allg. Chem., 569 (1989) 62.

215. T. Schoop, H.W. Roesky, M. Noltemeyer and H.G. Schmidt, Organometallics, 12 (1993) 571

216. T.M. Soliman, S.E.H. Etaiw, G. Fendesak and R.D. Fischer, J. Organometal. Chem., 415 (1991) C1.

217. S.F. Watkins, J. Chem. Soc. A, (1969) 1552.

218. S.G. Bott, J.C. Marchell, D.M.P. Mingos and M.J. Watson, J. Chem. Soc., Dalton Trans., (1991) 
219. H.J. Haupt, T. Keppler, U. Flörke and Y. Calage, Z. Anorg. Allg. Chem., 571 (1989) 51.

220. K. Merzweiler and H. Kraus, Z. Naturforsch., $48 \mathrm{~b}$ (1993) 1009.

221. G.K. Campbell, P.B. Hitchcock, M.F. Lappert and M.C. Misra, J. Organometal. Chem., 289 (1985) Cl

222. H. Schmidbaur, T. Probst, O. Steigelmann and G. Müller, Z. Naturforsch., 44b (1989) 1175.

223. K. Merzweiler and H. Kraus, $Z$. Naturforsch., 49b (1994) 621.

224. A. Müller, I. Paulat-Böschen, B. Krebs and H. Dornfeld, Angew. Chem. Int. Ed. Engl., 15 (1976) 633.

225. Ying-Jie Lu and J.A. Ibers, Acta Crystallogr., Sect. C, 47 (1991) 1600.

226. K.D. Bos, E.J. Bulten and J.G. Noltes, J. Organometal. Chem., 92 (1975) 33.

227. H. Preut and H.J. Haupt, Z. Anorg. Allg. Chem., 422 (1976) 47.

228 .

229.

230.

231.

232.

233.

234.

235.

236.

237.

238.

239.

B. Schiemenz, G. Huttner, L. Zsolnai, P. Kircher and T. Diercks, Chem. Ber., 128 (1995) 187.

A.L. Spek, K.D. Bos, E.J. Bulten and J.G. Noltes, Inorg. Chem., 15 (1976) 339.

K. Merzweil and L. Weisse, Z. Naturforsch., 45b (1990) 1971.

K. Jurkschat, C. Kruger and J. Meunier-Piret, Main Group Met. Chem., 15 (1992) 61.

K. Merzweiler, H. Kraus and L. Weisse, Z. Naturforsch., 48b (1993) 287.

H.J. Haupt, P. Balsaa, B. Schwab, U. Flörke and H. Preut, Z. Anorg. Altg. Chem., 513 (1984) 22.

H. Preut, H.J. Haupt and U. Flörke, Acta Crystallogr., Sect. C, 40 (1984) 600.

D.M.P Mingos, H.R. Powell and T.L. Stolberg, Transition Met. Chem., 17 (1992) 334.

S.G. Anema, K.M. Mackay and B.K. Nicholson, J. Organometal. Chem., 372 (1989) 25.

P. Ewing and L.J. Farrugia, New J. Chem., 12 (1988) 409.

A.L. Balch, M.M. Olmstead and D.P. Oram, Inorg. Chem., 27 (1988) 4309.

L.R. Sita, Rimo Xi, G.P.A. Yap, L.M. Liable-Sands and A.L. Rheingold, J. Amer. Chem. Soc., 119 (1997) 756.

240.

241.

242.

243.

244.

245.

246.

247.

248.

249.

B. Schiemenz, F. Ettel, G. Huttner and L. Zsolnai, J. Organometal. Chem., 458 (1993) 159.

R.M. Schweet, C.J. Fritchie Jr. and R.A. Schunn, Inorg. Chem., 6 (1967) 749.

C.J. Cardin, M.B. Power and D.J. Cardin, J. Organometal. Chem., 462 (1993) C27.

T.J. McNeese, S.S. Wreford, D.L. Tipton and R. Bau, J. Chem. Soc., Chem Commun., (1977) 390.

O.J. Curnow and B.K. Nicholson, J. Organometal. Chem., 267 (1984) 257.

F.A. Cotton and M. Shang, Inorg. Chem., 29 (1990) 2614.

H. Akashi and T. Shibahara, Inorg. Chem., 28 (1989) 2906.

M. Ferrer, A. Perales, O. Rossell and M. Seco, J. Chem. Soc., Chem. Commun., (1990) 1447.

U. Flörke and H.J. Haupt, Z. Kristallogr., 201 (1992) 301.

250. K. Schmidbaur, T. Probst, B. Huber, G. Müller and C. Krüger, J. Organometal. Chem., 365 (1989) 53.

251. C. Chandler, G.D. Fallon and B.O. West, J. Chem Soc., Chem. Commun., (1990) 1063.

252. M.S. Rau, C.M. Kutz, G.L. Geoffrey, A.L. Rheingold and B.S. Haggerty, Organometallics, 13 (1994) 1624.

253. R.M. Harker, M.F. Mahon and K.C. Molloy, Main Group Met. Chem., 19 (1996) 29.

254. S.G. Anema, K.M. Mackay and B.K. Nicholson, Inorg. Chem., 28 (1989) 3158.

255. G.J. Spivak, L. Hao, J.J. Vittal and R.J. Puddephatt, J. Amer. Chem. Soc., 118 (1996) 225.

256. R.C. Burns and J.D. Corbett, J. Amer. Chem. Soc., 104 (1982) 2804.

257. L.N. Bochkarov, O.V. Grachev, N.E. Molosnova and S.F. Zhiltsov, J. Organometal. Chem., 443 (1993) C26.

258. Z. Demidowicz, R.L. Johnston, J.C. Machell, D.M.P Mingos and I.D. Williams, J. Chem. Soc., Dalton Trans., (1988) 1751.

259. B.W. Eichborn, R.C. Haushalter and W.T. Pennington, J. Amer. Chem. Soc., 110 (1988) 8704.

260. M.H. Chisholm, Hsin-Tien Chiu., K. Folting and J.C. Huffman, Inorg. Chem., 23 (1984) 4097: M.J. Chetcuti, M.H. Chisholm, Hsin-Tien Chiu and J.C. Huffman, J. Amer. Chem. Soc,, 105 (1983) 1060.

261. K. Merzweiler and L. Weisse, Z. Naturforsch., 46b (1991) 695.

262. B. Schiemenz and G. Huttner, Angew. Chem. Int. Ed. Engl., 32 (1993) 297.

263. E. Brivio, A. Ceriotti, L. Garlashelli, M. Manassero and M. Sansoni, J. Chem Soc., Chem. Commun., (1995) 2055.

264. Feibo Xin, M.T. Pope, G.J. Long and U. Russo, Inorg. Chem., 35 (1996) 1207.

265. J.P. Zebrowski, R.K. Hayashi and L.F. Dahl, J. Amer. Chem. Soc., 115 (1993) 1142.

266. A. Ceriotti, F. Demartin, B.T. Heaton, P. Ingallina, G. Longoni, M. Manassero, M. Marchionna and N. Masciochi, J. Chem. Soc., Chem. Commun., (1989) 786.

267. Feibo Xin and M.T. Pope, Organometallics, 13 (1994) 4881.

268. M. Veith and M. Reimers, Chem. Ber., 123 (1990) 1941.

269. J.C. Dewan, J. Silver, J.D. Donaldson and M.J.K. Thomas, J. Chem. Soc., Dalton Trans., (1977) 
2319.

270. M.S. Weininger, P.F. Rodesiler and E.L. Amma, Inorg. Chem., 18 (1979) 751.

271. G. Schmidbaur, T. Probst, O. Steigelmann and G. Müller, Heteroatom. Chem., 1 (1990) 161.

272. P.F. Rodesiler, T. Anel and E.L. Amma, J. Amer. Chem. Soc., 97 (1975) 7405.

273. W. Frank, Chem., Ber., 123 (1990) 1233.

274. M.J. Hampden-Smith, D.E. Smith and E.N. Duesler, Inorg. Chem., 28 (1989) 3999.

275. A.K. Brimah, E. Siebel, R.D. Fischer, N.A. Davies, D.C. Apperley and R.K. Harris, J. Organometal. Chem., 475 (1994) 85.

276. J. Lu, W.T.A Harrison and A.J. Jacobson, Angew, Chem. Int. Ed. Engl., 34 (1995) 2557.

277. Y. Sazaki, H. Imoto and O. Nagano, Bull. Chem. Soc. Jpn., 57 (1984) 1417.

278. U. Behrens, A.K. Brimah, K. Yünlii and R.D. Fischer, Angew, Chem. Int. Ed. Engl., 32 (1993) 82.

279. B. Kanellakopulos, K. Raptis, B. Nuber and M.L. Ziegler, Z. Naturforsch., 46b (1991) 15.

280. E. Herdtweck, P. Kiprof, W.A. Herrmann, J.G. Kuchler and I. Degnan, Z. Naturforsch., 45b (1990) 937.

281. U. Behrens, A.K. Brimah and R.D. Fischer, J. Organometal. Chem., 411 (1991) 325.

282. M. Adams, A.K. Brimah, R.D. Fischer and Li Xing-Fu, Inorg. Chem., 29 (1990) 1595.

283. P. Schwarz, E. Siebel, R.D. Fischer, D.C. Apperley, N.A. Davies and R.K. Harris, Angew. Chem. Int. Ed. Engl., 34 (1995) 1197.

284. R. Graziani, U. Casellato and G. Plazzogna, J. Organometal. Chem., 187 (1980) 381.

285. K. Yünlii, N. Hock and R.D. Fischer, Angew. Chem. Int. Ed. Engl., 24 (1985) 879.

286. U. Behrens, A.K. Brimah, T.M. Sollman, R.D. Fischer, D.C.Apperley, N.A. Davies and R.K. Harris, Organometallics, 11 (1992) 1718.

287. S. Eller, M. Adam and R.D. Fischer, Angew. Chem., Int. Ed. Engl., 29 (1990) 1126.

288. E. Siebel and R.D. Fischer, Chem. Ber., 3 (1997) 1987.

289. F.J. Hollander, M. Leitheiser and D. Coucouvanis, Inorg. Chem., 16 (1977) 1615.

290. S.S. Dhingra, Dong-Kyun Seo, G.R. Kowach, R.K. Kremer, J.L. Shreeve-Keyer, R.C. Haushalter and Myung-Hwa Whangbo, Angew. Chem. Int. Ed. Engl., 36 (1997) 1067.

291. Chwanchin Wang and R.C. Haushalter, J. Chem. Soc., Chem. Commun., (1997) 1719.

292. B. Binder, J. Inorg. Nucl. Chem., 41 (1979) 257.

293. C.E. Holloway and M. Melnik, Main Group Met. Chem., 20 (1997) 107.

294. C.E. Holloway and M. Melnik, Main Group Met. Chem., 20 (1997) 399. 